\title{
Micro-Macro and jamming transition in granular materials
}

\section{Nishant Kumar}

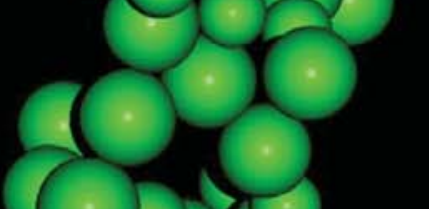




\section{Micro-Macro and jamming transition in granular materials}

Nishant Kumar 


\section{Samenstelling promotiecommissie :}

Prof. dr. Geert P.M.R. Dewulf (voorzitter) Universiteit Twente

Prof. dr. rer.-nat. Stefan Luding (promotor) Universiteit Twente

Dr. Vanessa Magnanimo (assistant promotor) Universiteit Twente

Prof. dr. Jin Ooi

Prof. dr. Devaraj van der Meer

University of Edinburgh

Prof. dr. ir. André de Boer

Universiteit Twente

Dr. Catherine O'Sullivan

Universiteit Twente

Dr. Fatih Göncü

Imperial College London

Yıldırım Beyazıt Üniversity

\section{UNIVERSITY OF TWENTE. T IIST: :: PARPEM}

The work in this thesis was carried out at the Multi Scale Mechanics (MSM) group of the Faculty of Science and Technology of the University of Twente. It is part of the research program PARDEM, which is financially supported by the European Union funded Marie Curie Initial Training Network, FP7 (ITN-238577).

Nederlandse titel :

Micro-macro en jamming overgang in granulaire materialen

Cover design : Nishant Kumar, Vitaliy Ogarko (Software), Abhinendra Singh and Shushil Kumar (Design and Implementation)

(Back to front) Transition of percolating strong force network from unjammed, to fragile and finally leading to a shear jammed state.

Publisher :

Nishant Kumar, Multi Scale Mechanics, University of Twente,

P.O. Box 217, 7500 AE Enschede, The Netherlands

Web : http://www . utwente.nl/ctw/msm/

Email : n.kumar@utwente.nl

Printer : Gildeprint, Enschede

(C) Nishant Kumar, Enschede, The Netherlands, 2014

No part of this work may be reproduced by print, photocopy or any other means without the permission in writing from the publisher

ISBN : 978-90-365-3634-9

DOI : 10.3990/1.9789036536349 


\title{
MICRO-MACRO AND JAMMING TRANSITION \\ IN GRANULAR MATERIALS
}

\author{
PROEFSCHRIFT
}

ter verkrijging van

de graad van doctor aan de Universiteit Twente,

op gezag van de rector magnificus,

prof. dr. H. Brinksma,

volgens besluit van het College voor Promoties

in het openbaar te verdedigen

op vrijdag 14 maart 2014 om 12.45 uur

door

Nishant KUMAR

geboren op 06 Juli 1987

te Allahabad, India 
Dit proefschrift is goedgekeurd door de promotor:

Prof. dr. rer.-nat. Stefan Luding

en de assistent-promotor:

Dr. Vanessa Magnanimo 
Dedicated to my family and friends 



\section{Summary}

\section{Micro-Macro and jamming transition in granular materials}

by N. Kumar

Granular materials are prevalent ubiquitously in nature and everyday life. They are commonly used as raw materials in various industries; their processing, handling and storage is far from understood, posing many open challenges for scientific community. Granular materials behave in a different manner than usual solids and fluids, exhibit unique mechanical properties. For example, granular matter can flow when shaken or poured through a hopper, but jams (solidifies) when the shaking intensity or pouring rate is lowered. For these reasons, they have attracted significant scientific interest over the past few decades. The bulk behavior of granular materials depends on their constituent particles and they interact through contact forces. The major objective of this work is to model the micro-macro transition towards understanding their micro-based macro-behavior.

In the first part of this dissertation, simulational results using the Discrete Element Method (DEM) of idealized, frictionless, disordered sphere packings of dense granular materials are presented. The goal is to gain a better understanding of the mechanical behavior of granular matter. A guideline is presented for calibrating a simplified theoretical anisotropy continuum model using the results from isotropic and deviatoric element tests. This calibrated model (parameters) is then able to predict qualitatively the macroscopic behavior of granular assemblies for an independent uniaxial compression test. Afterwards, the micro- and macro-mechanical behavior of similar assemblies emphasizing the effect of polydispersity is analyzed. As main finding, a relationship for the jamming volume fraction (and other parameters) as functions of the polydispersity and the deformation modes is obtained.

The goal of the second part of this dissertation, is to link the elastic moduli (small strain stiffness) with the state variables of the polydisperse anisotropic material, in order to predict the constitutive macroscopic behavior along a generic deformation path. This is achieved by applying small perturbations to various static equilibrium states that previously expe- 
rienced different history, and by investigating the effect of volume fraction, stress state and microstructure (fabric) on the bulk elastic response of the material. A fully calibrated elasticplastic anisotropy constitutive model is the major result and is able to predict quantitatively the evolution of pressure, shear stress and deviatoric fabric for an independent cyclic pure shear test.

Finally, in the last chapter, based on the study of soft, frictionless, polydisperse spheres, a quantitative model is proposed for how the jamming density changes with history; this quantity is then representing a memory state-variable of the system. One can explain how the packing efficiency increases logarithmically slow under gentle "tapping" or repeated compression, and, in contrast, how it rapidly decreases for shear deformations. By modifying the anisotropy continuum model, and adding the memory (history) dependent jamming point, its predictive power is shown to quantitatively explain many more real-world observations. 


\section{Samenvatting}

\section{Micro-macro-en jamming overgang in granulaire materialen}

door N. Kumar

Granulaire materie komt overal voor in de natuur en in het dagelijkse leven en wordt vaak gebruikt als grondstof in verschillende industrieën. Het gedrag dat dit soort materialen vertoond, bijvoorbeeld tijdens verwerking en opslag, is echter nog lang niet volledig begrepen. Granulaire materie gedraagt zich als een vloeistof wanneer het geschud wordt of in een trechter wordt gegoten, echter wanneer de intensiteit van het schudden of de massastroom bij het gieten word verlaagd zullen de deeltjes klem komen ze zitten en gedraagt de materie zich meer als een vaste stof. Het macroscopische gedrag van granulaire materie hangt af van de deeltjes waaruit het is samengesteld. Deze deeltjes op hun beurt hebben interactie met elkaar door individuele botsingen. Het hoofddoel van dit werk is om de micro-macro overgang van granulaire materie te modelleren, zodat het macroscopische gedrag beschreven kan worden aan de hand van het microscopische gedrag.

In het eerste deel van dit proefschrift worden resultaten van Discrete Element Method (DEM) simulaties van geïdealiseerde, wrijvingsloze, wanordelijke verzamelingen van dicht gepakte deeltjes gepresenteerd. Het doel van deze simulaties is een beter begrip van het mechanische gedrag van granulaire materie verkrijgen. Een richtlijn voor het kalibreren van een vereenvoudigd, theoretisch, continuüm, model, met behulp van DEM simulaties van isotrope en deviatorische elementaire testen, wordt gegeven. Dit gekalibreerde model is vervolgens gebruikt om de resultaten van een onafhankelijke compressie test kwalitatief te voorspellen. Ook hebben we het micro- en macro-scopische gedrag van soortgelijke deeltjes samenstelling geanalyseerd, afhankelijk van de polydispersiteit in de grote van de deeltjes. We presenteren een relatie voor onder andere het volumepercentage waarop het systeem klem komt te zitten (zich als een vaste stof gaat gedragen) als functie van de polydispersiteit en deformatiemodes.

Het doel van het tweede deel van het proefschrift is om de elasticiteitsmoduli te koppelen 
aan de toestandsvariabelen van een polydispers, anisotroop materiaal, om het constitutieve gedrag van granulaire materie te voorspellen onder generieke vervormingen. Hiervoor zijn kleine verstoringen aangebracht op diverse statische evenwichtstoestanden van verzamelingen van granulaire deeltjes met verschillende vervormingsgeschiedenis en onderzoeken we het effect van het volumepercentage, de spanningstoestand en de microstructuur op de macroscopische elastische respons van het materiaal. Het hoofdresultaat is een volledige gekalibreerd anisotroop constitutief model, dat in staat is om de evolutie van de druk, schuifspanning en microstructuur voor een onafhankelijke cyclische zuivere afschuif test kwantitatief te voorspellen.

Tenslotte wordt in het laatste hoofdstuk, aan de hand van de studie van zachte, wrijvingsloze, polydisperse, bolvormige deeltjes, een kwantitatief model gepresenteerd voor het veranderen van het volumepercentage waarop het systeem tot stilstand komt, gebaseerd op de geschiedenis van het materiaal. Met dit model kan zowel worden uitgelegd hoe dit volumepercentage logaritmisch toeneemt onder voorzichtig tikken of herhaalde compressie als wel als hoe snel deze afneemt onder schuifvervormingen. Deze afhankelijkheid van het punt waarop het granulaire materiaal tot stilstand komt is toegevoegd aan het anisotrope constitutieve model. Met dit verbeterde model zijn verschillende werkelijke observaties kwantitatief uitgelegd. 


\section{Acknowledgements}

I still remember, back in 2010 when Marco Ramaioli, my supervisor during internship in Nestlé, recommended me for this PARDEM project titled "Micro-macro laws for new contact models in the field of Discrete Element Methods", and I applied for this opportunity without any hesitations. The whole appointment process, interviews, contract, travel to Enschede, everything happened quite quickly when I decided to join Stefan's group in August 2010 - knowing very little about how the research and everything would work here. And today, I want to express my deepest gratitude to the people who are involved in the successful completion of my $\mathrm{PhD}$.

In particular, I would like to thank:

Stefan, for the trust he has in me, for providing me the opportunity to work with him. I am thankful to him for explaining my questions lucidly. His way of dealing with problems at smaller levels and then adding levels of complexity to it, to combine various effects, was really productive and I found it an efficient way of working. He always welcomed new ideas and perspectives toward research and provided feedback through experience to show alternative directions and views. I highly appreciate the freedom he gave to me to work on this research topic, in choosing my own direction to achieve the research objectives, all of which made this experience extremely efficient and productive for me. I feel spoiled due to the freedom and flexibility he has afforded to me, and I believe I have learnt to value the productive work than the mundane office hours. To say the least, I have learned a lot from him; both professionally and personally. Thank you Stefan, for allowing me to attend many conferences, presenting my work and enjoyable vacations otherwise. I would like to also thank his wife Gerlinde, for all the group dinners and gatherings they hosted.

Vanessa, for being the best advisor I can imagine! She has been an excellent mentor for me, always full of enthusiasm, wisdom and experience - all of which made the collaboration a joyful experience. Many thanks to her for those coffee time discussions, when I could even discuss about non-scientific experiences, such as driving lessons. Thank you for your tremendous support, fruitful discussions and encouragement; I had a marvelous time 
working with you and look forward to collaborate with you in future.

Ankit, Pradeep, Megha and Ashish, for being the closest distant friends. I remember someone said "Distance makes heart grow fonder". I will always cherish the amazing time, including (many) memorable road trips, birthday celebrations, and much more we enjoyed together. Ankit, thank you for providing me all the motivation, the passion to work and at the same time fun, when I needed that. Pradeep and Megha, thank you for making me realize to be cool in difficult situations, and to believe in that its not the end, but just a new beginning. Ashish, for all your jokes, the discussions on diverse subjects and the good time. Pradeep many special thanks to you for taking out time to review and edit my thesis. A big thank you all for your regular visits to Enschede, and always making me believe that time can change the Calendars, but it cannot change true friends.

Abhi, has been a great colleague and a greater friend at UTwente. We spent together a lot of time together - working, making jokes and philosophical discussions in the office as well as during our meals. He was one of the few people I met when I first arrived in Enschede, and I adore the fun, as well as the serious conversations we had since then. Thank you for supporting and standing next to me in my good and bad times. I learnt a lot from him. Shruti, who was always eager to listen to my stories, answering my surplus curious questions, and understanding me - and of course, for the super amazing dinners, specially when I did not have energy to cook some for myself (almost always). I have had fascinating time with both Abhi and Shruti, in all of our trips together, the most memorable being Australia and Het Rutbeek. Ankit Verma, I had never imagined that he would be one of the closest relative to talk with about different subjects and for the loud laughs together. I have always looked forward to his visits to Enschede over the weekends, and we could never finish all the plans. Shushil, thank you for having many pointless, delightful discussions and for your delicious spicy food. Thank you for those deep and hours long conversations and the laughter, I will never forget that. The uncountable overnight cards game sessions, twenty nine, with Abhi, Ankit, Shushil and Shruti are something I will always remember. Hammad, I am very thankful for your excitement for tennis and playing many matches as team. Thank you Urooj for the delicious food. Giridhar, many thanks for helping me practice tennis regularly.

I would also like to thank my committee members for their interest in my research. In particular, I am very thankful to Fatih, for inspiring and guiding me during my PhD. Thank you Catherine, for accepting to be on my $\mathrm{PhD}$ committee at a short notice and for the critical reviews on my thesis and papers. I would also like to thank my close collaborators Itai Einav, Marco Ramaioli, Mario Liu, Jia Lin, Bob Behringer for their fruitful scientific discussions. The financial support from PARDEM, Marie Curie ITN, is gratefully acknowledged. I want to thank all the PARDEM supervisors, industrial partners and colleagues for giving a platform to work together on subjects that involved flow of ideas from both academia and the industry. 
I am very thankful to my former and present colleagues in the MSM group Martin Robinson, Mateusz Wojtkowski, Olukayode Imole, Deepak Tunuguntla, Brian Lawney, Abhinendra Singh, Dinant Krijgsman, Lisa de Mol, Stefan Emmerich, Fabian Uhlig, Sebastian Gonzalez, Anthony Thornton, Remco Hartkamp, Sudheshna Roy, Thomas Weinhart, Wouter den Otter, Kazem Yazdchi, Saurabh Srivastava, Vitaliy Ogarko, Kuniyasu Saitoh, Nicolás Rivas, Sylvia Hodes-Laarhuis and Wouter den Breeijen for the pleasant atmosphere in truly international environment. I thoroughly enjoyed working with them, and improved my research skills. Thank you Kay and Mateusz, for spending the easiest, coolest time we had in our PARDEM office. I learnt a lot from you guys on various topics. I cherish spending time with you, through many coffee breaks along the day, at the lake etc; times like that, I think I would never forget. Thank you Mateusz for your right away ready to help attitude, guiding me on Linux, C++, vi editor and working with several python codes needed for my data analysis. I can never forget our vacations in India, Nepal, Georgia, Armenia, and many in Europe and await for the upcoming ones. Thank you Sylvia, for being a friend, a mom, and a colleague at many different occasions. Any problem one has, no problem for Sylvia. Thank you for all the help and interest in talking to me. Thank you Wouter den Breeijen for helping with any computer related problems and being available when needed. I remember the time when my disc crashed, and you still managed to retrieve the data. Vitaliy, thank you for being a great friend and a companion. We got to know each other towards the end of our $\mathrm{PhD}$, when both of us started looking for job. The discussions about anything and everything are unforgettable, that started in San Francisco. Thank you for the time and memorable trips we have had together. Dinant, I have special gratitude for taking out your precious time for helping with the samenvatting of this thesis. I would also like to thank my friends in the Indian community here, specially Omkar, Rohit, Kartikeya, Naveen.

Finally, I wholeheartedly thank my family: my parents Mahendra and Indo, brother Prashant and sister Sristi, my grandparents and all my relatives. I can just say, I miss you. Prashant, thank you for the trust you have in me and supporting me to follow my dreams. I cannot imagine how things would be without those lessons on vector algebra. I really enjoyed all the trips together, we managed to make time for, in Europe. It brings smiles to my face, whenever I think about the our vacations in Enschede. My sister Sristi, thank you for listening to all my complicated stories, beautiful discussions and the care and warmth that you have provided. I want to thank everyone for coming from around the globe on this special occasion. There are many people, who are behind me on this day - my sincere apologies if I have forgotten to name someone, but I know you will always be there for me.

Nishant Kumar

Enschede, March 2014 


\section{Contents}

$\begin{array}{lll}\text { Summary } & \text { v }\end{array}$

Samenvatting vii

$\begin{array}{ll}\text { Acknowledgements } & \text { ix }\end{array}$

1 Introduction 1

1.1 What are granular materials? . . . . . . . . . . . . . 1

1.2 Need to study granular materials . . . . . . . . . . . . . . . 2

1.3 Discrete Element Methods and "micro-macro transition" . . . . . . . . . . 3

1.4 Continuum modeling . . . . . . . . . . . . . . . 4

1.5 Jamming in granular materials . . . . . . . . . . . . . . 5

1.6 Scope and Outline . . . . . . . . . . . . . . . 5

2 Behavior of granular assemblies under different paths 7

2.1 Introduction and Background . . . . . . . . . . . . . 8

2.2 Simulation method . . . . . . . . . . . . . . . . . . 10

2.3 Preparation and test procedure . . . . . . . . . . . . . 12

2.4 Averaged quantities . . . . . . . . . . . . . . . . . . . 15

2.5 Evolution of micro-quantities . . . . . . . . . . . . 20

2.6 Evolution of macro-quantities . . . . . . . . . . . . 26

2.7 Theory: Macroscopic evolution equations . . . . . . . . . . . . . . . 32

2.8 Conclusions and Outlook . . . . . . . . . . . . . . . 37

2.A Shape-factors $\zeta$, for the different deformation modes . . . . . . . . 41

3 Effects of polydispersity on the behavior of granular assemblies 43

3.1 Introduction and Background . . . . . . . . . . . . . . . 44

3.2 Numerical simulation . . . . . . . . . . . . . . . . . . . 45

3.3 Preparation and test procedure . . . . . . . . . . . . . . 48

3.4 Polydispersity . . . . . . . . . . . . . . . . . . . 48

3.5 Calibration of the continuum model and prediction . . . . . . . . 63 
3.6 Summary and Outlook . . . . . . . . . . . . . . . . . 69

3.A Table of parameters . . . . . . . . . . . . . . . 71

4 Constitutive model with anisotropy for granular materials $\quad 73$

4.1 Introduction . . . . . . . . . . . . . . . . . . . . . . . . . 74

4.2 Numerical simulation . . . . . . . . . . . . . . . . . . 76

4.3 Volume conserving (undrained) biaxial shear test . . . . . . . . . . . 79

4.4 Elastic moduli . . . . . . . . . . . . . . . . . . . . 83

4.5 Prediction of undrained cyclic shear test f . . . . . . . . . . . . . . 98

4.6 Summary and Outlook . . . . . . . . . . . . . . . . . . . . 102

4.A Elastic moduli for polydisperse granular materials . . . . . . . . . . . . . . 104

5 Memory of jamming and shear-jamming 109

5.1 Introduction and Background . . . . . . . . . . . . . . . . . 110

5.2 Cyclic isotropic over-compression . . . . . . . . . . . . . . 111

5.3 Shear jamming below $\phi_{J}(H) \ldots \ldots \ldots \ldots$. . . . . . . . . . . . . . . . . . . . . . . . .

5.4 Jamming phase diagram with history $H \ldots \ldots$. . . . . . . . . . . . . 114

5.5 Slow dynamics model . . . . . . . . . . . . . . . . . 116

5.6 Prediction: minimal model . . . . . . . . . . . . . . . . . . 117

5.7 Interpretation and Outlook . . . . . . . . . . . . . . . . . . 119

5.8 Methods . . . . . . . . . . . . . . . . . . . . . 120

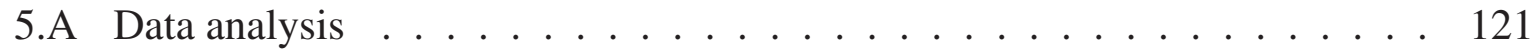

5.B Identification of the jamming point . . . . . . . . . . . . . . 121

5.C Isotropic cyclic over-compression . . . . . . . . . . . . . . . . . 122

5.D Effect of system size and initial configurations . . . . . . . . . . . . . 124

5.E Cyclic Shear results . . . . . . . . . . . . . . . . . . 125

5.F Percolation analysis . . . . . . . . . . . . . . . . 128

5.G Relaxation effects . . . . . . . . . . . . . . . . . 130

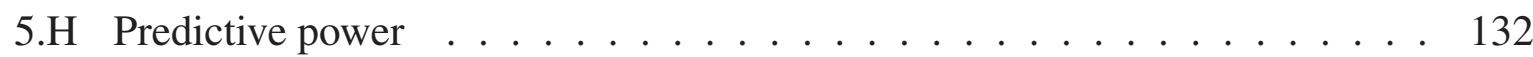

5.I How to measure $\phi_{J}$ from experiments $\ldots \ldots \ldots \ldots$

6 Conclusions and Recommendations 139

$\begin{array}{ll}\text { References } & 143\end{array}$

$\begin{array}{ll}\text { Curriculum vitae } & 155\end{array}$ 


\section{ChAPTER 1}

Introduction

\subsection{What are granular materials?}

Granular materials are apparently very simple: they are large assemblies of discrete macroscopic particles. In our day-to-day life, granular materials are regularly encountered. A general day starts with cereals and coffee beans in the morning, riding/driving on road or railway networks to office, eating M\&M's and hot chocolate (cocoa) in breaks, cooking meals in the evening with rice, salt peppers, spices, etc., all comprises of these materials, see Fig. 1.1. These are just few examples from our daily life where one can easily realize the usage and importance of granular materials. Processing, handling and storage of particle systems in the form of granular materials is widespread in all sectors of industry, and is beyond the scope of our imagination. They constitute over $75 \%$ of all raw material feedstock to process industries (chemical, pharmaceutical, building materials, food, power, textile, material, environmental protection or waste recycling industries, biotechnology, metallurgy, agriculture) as well as electronics. This includes plastic pellets, agricultural grains, coal and other minerals, pharmaceutical powders, sand, gravel, sugar and flour etc [156].

Despite its ubiquity and simplicity, the physics of granular media is poorly understood, posing an obstacle in industrial and geophysical applications. They present many challenges for innovation and fundamental science, to solve problems in areas as diverse as natural disasters and unsolved industrial material handling issues which incur extensive economic losses. Next some peculiar behavior of collective systems of granular materials is briefly presented and explained the need to study them. 


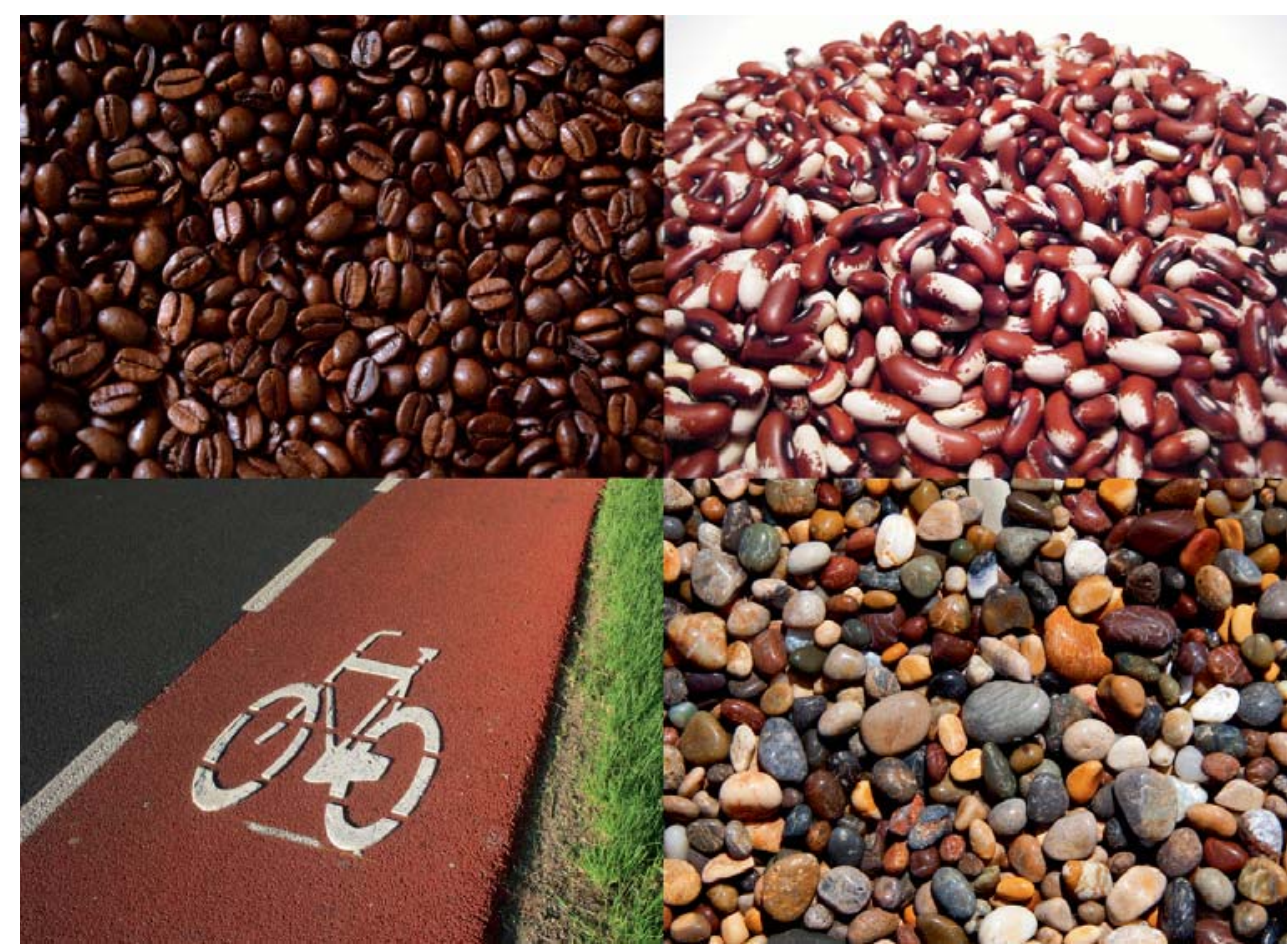

Figure 1.1: Few examples of granular matter in our daily life, taken from [1].

\subsection{Need to study granular materials}

The search for broad, general concepts that help to explain all mechanisms in collective systems of granular materials, is particularly appealing to physicists. Some of these mechanisms include inhomogeneity in systems, such as reflected by force-networks [162, 181, 185], over-population of weak/soft/slow mechanical oscillation modes [182], diverging correlation lengths and relaxation time-scales [23, 113, 150, 209], but also some universal behavior [32]. Other phenomena occur like shear-strain localization [153, 158, 200], anisotropic evolution of structure and stress [21, 38, 55, 84, 150, 158, 162, 182, 185, 209], clustering, shear-band formation, size segregation [168], arching [82], density waves, acoustic effects and pattern formation such as sand ripples and dunes [7] and oscillating mass flow rates [137].

Many industrial solid particle systems display unpredictable behavior and thus are difficult to handle, see Fig. 1.1. In an industrial survey, Ennis et al. [54] reported that $40 \%$ of the capacity of industrial plants is wasted because of granular solid problems. Merrow [138] found that the main factor causing long start-up delays in chemical plants is solids processing, especially the lack of reliable predictive models and simulations [156].

Attempts to model these systems with classical continuum theory and standard numerical methods and design tools cannot always be successful because they ignore the fact that particle systems consist of discrete objects. A promising interdisciplinary alternative is the 


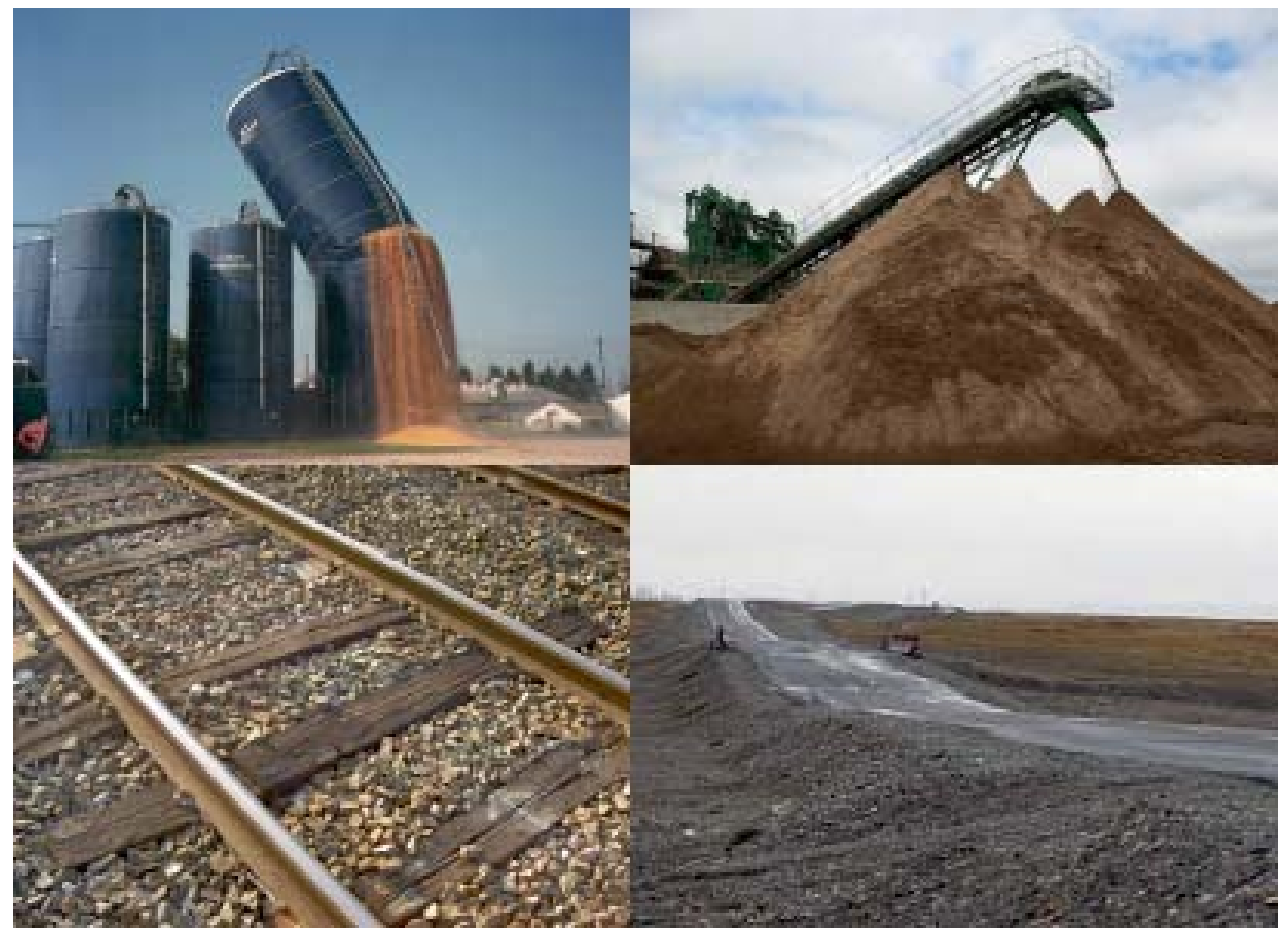

Figure 1.2: Some examples where the need to model granular materials is necessary (in clockwise direction) : Collapse of silo used for storing granular materials, Transportation of sand using conveyer belt, Granular ratcheting due to deformation in supportive granular rail bed in railway tracks, and on road networks, taken from [2].

multiscale approach, where the behavior at macroscale is linked to the kinematic of the particles at smaller scale. Both fundamental understanding and design/operation of unitprocesses and plants thus require a multiscale and multiphase approach, where the discrete nature of the particles is of utmost relevance and must not be ignored [191]. In such a framework, Discrete Element Method (DEM) becomes a perfect tool to gain insight into the microscopic evolution, as it follows in detail the motion and interaction of the single particles, as discussed next.

\subsection{Discrete Element Methods and "micro-macro transi- tion"}

A possibility to obtain information about the behavior of granular media is to perform controlled experiments. In this respect, particle simulations are relatively recent powerful tools that allow to track individual particles with complex interaction by solving Newton's laws of motion. The most prominent discrete approach is the Discrete Element Method (DEM) which was introduced and applied in the field of geotechnics [42] and was later taken up as a research tool. In recent decades, the Discrete Element Method (DEM) has been used and 
advanced extensively for scientific purposes and has increased rapidly our understanding of both macroscopic and microscopic behavior [79, 201].

Next, an essential question is: how to bridge the gap between the microscopic picture (kinetic theory or molecular dynamics simulations of fluids or particulate flows) and a macroscopic description on the level of a continuum theory. The former involves impulses/contact-forces and collisions/deformations, whereas the latter concerns tensorial quantities like the stress or the velocity gradient.

The approach towards the microscopic understanding of macroscopic particulate material behavior is the modeling of particles using DEM. The recently developed so-called micromacro transition procedures aim at a better understanding of the macroscopic fluid/powder flow behavior based on microscopic foundations. Besides the experimental verification of the simulation results [112], the formulation of constitutive relations in the framework of continuum theory is the great challenge.

\subsection{Continuum modeling}

When realistic numbers of particles with complex geometries are considered, DEM simulations are very slow and continuum models are more desirable. Continuum models consider grain assemblies in granular media as a continuum domain by assuming that the grains have infinitesimal size. The discontinuities of variables at the microscopic scale are disregarded and the mechanical behavior of the material is presented as a constitutive relation, which is usually based on continuum mechanics with phenomenological hypotheses. The bulk behavior of particulate materials depends on the behavior of their constituents (particles). To get an understanding of their behavior, laboratory element tests can be performed. While such macroscopic experiments are important in developing constitutive relations, they provide little information on the microscopic origin of the bulk flow behavior of these complex packings.

Micro-mechanical based constitutive models can be derived from DEM simulations [59, $123,194]$ due to the detailed insight on particle positions, orientations and velocities. Great progress has been achieved in recent years, however, there is no standard approach available $[27,58]$ on how to measure input parameters, and how to validate DEM simulation results.

Although micromechanically based constitutive laws are being developed for granular materials $[63,205]$, the discussion is still open on how macroscale state variables such as stress and strain measures can be related to microscale quantities [11, 14, 31, 51, 98] among others. In particular, recent works $[3,73,94,110,222]$ show that along with the macroscopic properties (stress and volume fraction) [56, 94, 192, 219], also the structure, as quantified by the fabric tensor $[120,146,178,222]$ plays a crucial role, as it characterizes, on average, the geometric arrangement of contacts. Many standard constitutive models, involving elasticity 
and/or plasticity have been applied to describe the incremental behavior of granular solids sometimes with success, but typically only in a limited range of parameters. In the majority of the models, the stress increment is related to the actual stress state of the granular system and its density. This is the case for hypoplasticity [64, 68, 94, 192], where a single non-linear tensorial equation relates the Jaumann stress-rate with strain-rate and stress tensors. Later versions [145] also involve an explicit relation with the structure of the contact network. Only a few theories, see e.g. [29, 43, 64, 142, 143, 189, 193, 208] and references therein, consider explicitly the influence of the micro-mechanical structure on the elastic stiffness, plastic flow-rule or non-coaxiality of stress and strain. In a similar fashion, the anisotropy model proposed in $[128,131]$ postulates the split of isotropic and deviatoric stress, strain and fabric and includes the microstructural anisotropy as a kinematic variable, assumed to be independent of stress, whose behavior is described by an evolution equation.

\subsection{Jamming in granular materials}

Granular matter can flow through a hopper when shaken or agitated, but jam (solidify) when the shaking intensity is lowered [207]. Jamming is the physical process by which some materials, such as granular materials, glasses, foams, and other complex fluids, become rigid with increasing density. The jamming transition has been proposed as a new type of phase transition, with similarities to a glass transition but very different from the formation of crystalline solids [22].

To gain better understanding of the jamming transition concept, one needs to access the evolution of both the structure and the contact forces near the jamming transition. Both illustrate the transition, e.g. with a strong force chain network percolating the full system, thus making the unstable packing permanently stable and rigid [21, 220]. Full access is possible experimentally only in two-dimensional (2D) model systems [21, 46, 77, 220], with little progress in 3D [25, 47, 92, 139].

It is often assumed that such materials jam at a certain solid (packing) fraction [13, 116, 200], i.e., they become mechanically stable with finite bulk- and shear-moduli [139, 150, 160]. However, the notion of an a-thermal jamming "point" was recently challenged by report on shear-jamming regime [21, 38, 149, 220] slightly below the traditional (isotropic) jamming point, whereby application of shear strains can jam these states. This suggests the existence of a broad range of $\phi_{J}$, even for a given material [12, 32, 117, 134, 148, 151-155, 198].

\subsection{Scope and Outline}

To gain more insight into the micro-structure of granular materials to understand their micromacro and jamming transition, numerical studies and simulations are performed on various quasi-static deformation experiments. Thus, this thesis is divided in chapters covering the 
micro-macro description of granular materials and considers many aspects such as jamming, polydispersity, and constitutive modeling. Here the contents of the chapters are outlined.

In Chapter 2, the micromechanical and macromechanical behavior of idealized granular assemblies are studied, comprising linearly elastic, frictionless, polydisperse spheres, in a periodic, triaxial box geometry, using DEM. The stress- and structure-anisotropy (bulk) responses to various deformation modes applied to this granular assemblies are analyzed, namely purely isotropic and deviatoric (volume conserving) and a mixed, uniaxial deformation mode. Initially, a guideline is formulated for calibrating a simplified theoretical model with DEM simulations of isotropic and deviatoric element tests and then to predict another element test with this calibrated model (parameters).

Later in Chapter 3, the micromechanical and macromechanical behavior of similar assemblies are analyzed, emphasizing the effect of polydispersity under the three deformation modes. A relation for the jamming volume fraction (and other parameters) is presented as function of the polydispersity and the deformation mode. It is confirmed that the concept of a single jamming point has to be rephrased to a "range" of values, dependent on the microstructure and history of the sample. In both Chapters 2 and 3, the calibration of the microscopic simulation results with a short review of an simplified anisotropy continuum model is presented, as introduced in [128], together with a prediction of an independent test, i.e. the uniaxial deformation mode

Next, in Chapter 4, a link between the elastic moduli (small strain stiffness) with the state variables of the polydisperse anisotropic material is established. Small perturbations are applied to various static equilibrium states that previously experienced different finite/large pure shear strains and the effect of volume fraction, stress state and microstructure (fabric tensor) on the elastic bulk response of the material is investigated. Finally, an anisotropic constitutive model is calibrated that is able to predict quantitatively the evolution of pressure, shear stress and deviatoric fabric for an independent cyclic pure shear tests. The effect of (uniform) polydispersity on the elastic moduli is also addressed to complete the discussion on full macroscopic description of polydisperse materials.

In Chapter 5, the nature and structural origin of both jamming and shear-jamming in threedimensions is explained and a quantitative model is proposed for how the jamming density changes with the sample's history. This explains both: how the packing efficiency increases logarithmically slow under gentle "tapping" or repeated compression, and the shear deformations that, in contrast, rapidly decrease the jamming point, which is the only necessary ingredient that explains shear-jamming. All this can be explained by a universal picture involving a multi-scale, fractal-type energy landscape. Finally, by modifying the anisotropy continuum model, adding a memory (history) dependent jamming point to it, its predictive power is presented to quantitatively explain the many real-world observations.

The thesis ends with conclusions and recommendations for future research in Chapter 6. 


\title{
CHAPTER 2
}

\section{Behavior of granular assemblies under different paths ${ }^{*}$}

\begin{abstract}
Stress- and structure-anisotropy (bulk) responses to various deformation modes are studied for dense packings of linearly elastic, frictionless, polydisperse spheres in the (periodic) triaxial box element test configuration. The major goal is to formulate guidelines for the procedure of how to calibrate a theoretical model with discrete particle simulations of selected element tests and then to predict another element test with this calibrated model.

Only the simplest possible particulate model-material is chosen as the basic reference example for all future studies that aim to quantitatively model more realistic frictional, cohesive powders. Seemingly unrealistic materials are used to exclude effects that are due to contact non-linearity, friction, and/or non-sphericity. This allows to unravel the peculiar interplay of micro-structural organization, i.e. fabric, with stress and strain.

Different elementary modes of deformation are isotropic, deviatoric (volume-conserving), and their superposition, e.g., a uniaxial compression test. (Other ring-shear or stresscontrolled (e.g. isobaric) element tests are referred to, but not studied here.) The
\end{abstract}

*. Based on O. I. Imole, N. Kumar, V. Magnanimo, and S. Luding. Hydrostatic and Shear Behavior of Frictionless Granular Assemblies Under Different Deformation Conditions. KONA Powder and Particle Journal, 30:84108,2013 . The contribution of the first two authors to this work were equal in relation to the data analysis and writing the manuscript. 
deformation modes used in this study are especially suited for the biaxial and triaxial box element test set-up and provide the foundations for powder flow in many other experimental devices. The qualitative phenomenology presented here is expected to be valid, even more clear and magnified, in the presence of non-linear contacts, friction, non-spherical particles and, possibly, even for strong attractive/adhesive forces.

The scalar (volumetric, isotropic) bulk properties, like the coordination number and the hydrostatic pressure, scale qualitatively differently with isotropic strain, but behave in a very similar fashion irrespective of the deformation path applied. The deviatoric stress response, i.e., the stress-anisotropy, is proportional to deviatoric strain, as well as being cross-coupled to the isotropic mode of deformation via the structural anisotropy; likewise, the evolution of pressure is coupled via the structural anisotropy to the deviatoric strain. Note that isotropic/uniaxial over-compression or pure shear slightly increase or reduce the jamming volume fraction, respectively. This observation allows to generalize the concept of "the" jamming volume fraction, below which the packing loses mechanical stability, from a single value to a "wide range”, as a consequence of the deformation-history of the granular material that is "stored/memorized" in the structural anisotropy.

The constitutive model with incremental evolution equations for stress and structural anisotropy takes the above-mentioned phenomena into account. Its material parameters are extracted from discrete element method (DEM) simulations of isotropic and deviatoric (pure shear) modes as volume fraction dependent parameters. Based on this calibration, the theory is able to predict qualitatively (and to some extent also quantitatively) both the stress and fabric evolution in the uniaxial, mixed mode during compression.

\subsection{Introduction and Background}

Dense granular materials are generally complex systems which show unique mechanical properties that are different from those of classical fluids or solids. Interesting phenomena like dilatancy, shear-band formation, history-dependence, jamming and yield stress - among others - have attracted significant scientific interest over the past decade. The bulk behavior of these materials depends on the behavior of their constituents (particles) interacting through contact forces. To get an understanding of the deformation behavior of these materials, various laboratory element tests can be performed $[140,172,176]$. Element tests are (ideally homogeneous) macroscopic tests in which the experimentalist can control the stress and/or strain path. Different element test experiments on packings of bulk solids have been realized in the biaxial box (see [141] and references therein) while other deformations modes, namely uniaxial and volume conserving shear have been reported in [170]. While such macroscopic experiments are important ingredients in developing constitutive relations, they provide little 
information on the microscopic origin of the bulk flow behavior of these complex packings.

The complexity of the packings becomes evident when they are compressed isotropically. In this case, the only macroscopic control parameters are volume fraction and pressure [69, 128]. At the microscopic level for isotropic samples, the micro-structure (contact network) is classified by the coordination number (i.e. the average number of contacts per particle) and the fraction of rattlers (i.e. fraction of particles that do not contribute to the mechanical stability of the packing) [69]. However, when the same material sample is subjected to shear deformation, not only does shear stress build up, but also the anisotropy of the contact network develops, as it relates to the creation and destruction of contacts and force chains $[5,161,205]$. For anisotropic samples, scalar quantities are not sufficient to fully represent the internal contact structure, but an extra tensorial quantity has to be introduced, namely the fabric tensor [66]. To gain more insight into the micro-structure of granular materials, numerical studies and simulations on various deformation experiments can be performed, see Refs. [195-197] and references therein.

In an attempt to classify different deformation modes, Luding et al. [128] listed four different deformation modes: (0) isotropic (direction-independent), (1) uniaxial, (2) deviatoric (volume conserving) and (3) biaxial/triaxial deformations. The former are purely straincontrolled, while the latter (3) is mixed strain-and-stress-controlled either with constant side stress [128] or constant pressure [131]. The isotropic and deviatoric modes 0 and 2 are pure modes, which both take especially simple forms. The uniaxial deformation test is a superposition of an isotropic and a deviatoric test, and represents the simplest element test experiment (oedometer, uniaxial test or lambda-meter) that activates both isotropic and shear deformation. The biaxial tests are more complex to realize and involve mixed stressand strain-control instead of completely prescribed strains as often applied in experiments $[141,217]$, since they are assumed to better represent deformation under realistic boundary conditions - namely the material can expand and form shear bands.

In this study, various deformation paths for assemblies of polydisperse packings of linearly elastic, non-frictional cohesionless particles are modeled using the DEM simulation approach. One goal is to study the evolution of pressure (isotropic stress) and deviatoric stress as functions of isotropic and deviatoric strain. Microscopic quantities like the coordination number, the fraction of rattlers, and the fabric tensor are reported for improved microscopic understanding. Furthermore, the extensive set of DEM simulations is used to calibrate the anisotropic constitutive model, as proposed in Refs. [128, 131]. After calibration through isotropic [69] and volume conserving pure shear simulations, the derived relations between the parameters and volume fraction are used to predict uniaxial deformations. Another goal is to improve the understanding of the macroscopic behavior of bulk particle systems and to guide further developments of new theoretical models that describe it.

The focus on these seemingly unrealistic materials allows exclusion of effects that are due to 
friction, other contact non-linearities and/or non-sphericity, with the goal of unravelling the interplay of micro-structural organization, fabric, stress and strain. This is the basis for the present research that aims at the quantitative modeling of these phenomena and effects for realistic frictional, cohesive powders. The deformation modes used in this study are especially suited for the biaxial box experimental element test set-up and provide the fundamental basis for the prediction of many other experimental devices. The qualitative phenomenology presented here is expected to be valid, even more clear and magnified, in the presence of friction and non-spherical particles, and possibly even for strong attractive forces.

This chapter is organized as follows: The simulation method and parameters used are presented in section 2.2, while the preparation and test procedures are introduced in section 2.3. Generalized averaging definitions for scalar and tensorial quantities are given in section 2.4 and the evolution of microscopic quantities is discussed in section 2.5. In section 2.6, the macroscopic quantities (isotropic and deviatoric) and their evolution are studied as functions of volume fraction and deviatoric (shear) strain for the different deformation modes. These results are used to obtain/calibrate the macroscopic model parameters. Section 2.7 is devoted to theory, where we relate the evolution of the micro-structural anisotropy to that of stress and strain, as proposed in Refs. [128, 131], to display the predictive quality of the calibrated model.

\subsection{Simulation method}

The Discrete Element Method (DEM) [42], was used to perform simulations in biaxial and triaxial geometries [50, 99, 120, 190], involving advanced contact models for fine powders [122], or general deformation modes, see Refs. [5, 195, 197] and references therein. However, since we restrict ourselves to the simplest deformation modes and the simplest contact model, and since DEM is otherwise a standard method, only the contact model parameters and a few relevant time-scales are briefly discussed - as well as the basic system parameters.

\subsubsection{Force model}

For the sake of simplicity, a linear visco-elastic contact model has been used to simulate for the normal component of force in this work and friction was set to zero (and hence neither tangential forces nor rotations are present). This normal contact force model, which takes into account excluded volume and dissipation, involves a linear repulsive and a linear dissipative force, given as

$$
\mathbf{f}^{n}=f^{n} \hat{\mathbf{n}}=(k \delta+\gamma \dot{\delta}) \hat{\mathbf{n}},
$$

where $k$ is the spring stiffness, $\gamma$ is the contact viscosity parameter and $\delta$ or $\dot{\delta}$ are the overlap or the relative velocity in the normal direction $\hat{\mathbf{n}}$. An artificial viscous background dissipation force $\mathbf{f}_{b}=-\gamma_{b} \mathbf{v}_{i}$ proportional to the moving velocity $\mathbf{v}_{i}$ of particle $i$ is added, resembling 
the damping due to a background medium, as e.g. a fluid. The background dissipation only leads to shortened relaxation times, reduced dynamical effects and consequently lower computational costs without a significant effect on the underlying physics of the process - as long as quasi-static situations are considered.

The results presented in this study can be seen as the fundamental case for more realistic material models, see e.g. Ref. [122] and references therein. The interesting, complex behavior and non-linearities cannot be due to the contact model but due to the collective bulk behavior of many particles, as will be shown below.

\subsubsection{Simulation Parameters and time-scales}

Typical simulation parameters for the $N=9261\left(=21^{3}\right)$ particles with average radius $\langle r\rangle=$ $1[\mathrm{~mm}]$ are density $\rho=2000\left[\mathrm{~kg} / \mathrm{m}^{3}\right]$, elastic stiffness $k=10^{8}\left[\mathrm{~kg} / \mathrm{s}^{2}\right]$ particle damping coefficient $\gamma=1[\mathrm{~kg} / \mathrm{s}]$, and background dissipation $\gamma_{b}=0.1[\mathrm{~kg} / \mathrm{s}]$. The polydispersity of the system is quantified by the width $\left(w=r_{\max } / r_{\min }=3\right)$ of a uniform distribution with a step function as defined in [69], where $r_{\max }=1.5[\mathrm{~mm}]$ and $r_{\min }=0.5[\mathrm{~mm}]$ are the radius of the biggest and smallest particles respectively. Note that the units are artificial; Ref. [122] provides an explanation of how they can be consistently rescaled to match quantitatively the values obtained from experiments (due to the simplicity of the contact model used).

A typical response time is the collision duration $t_{c}$. For a pair of particles with masses $m_{i}$ and $m_{j}, t_{c}=\pi / \sqrt{k / m_{i j}-\left(\gamma / 2 m_{i j}\right)^{2}}$, where $m_{i j}=m_{i} m_{j} /\left(m_{i}+m_{j}\right)$ is the reduced mass. The coefficient of restitution for the same pair of particle is expressed as $e=\exp \left(-\gamma t_{c} / 2 m_{i j}\right)$ and quantifies dissipation. The contact duration $t_{c}$ and restitution coefficient $e$ are dependent on the particle sizes and since our distribution is polydisperse, the fastest response time scale corresponding to the interaction between the smallest particle pair in the overall ensemble is $t_{c}=0.228[\mu \mathrm{s}]$ and $e$ is 0.804 . For two average particles, $t_{c}=0.643[\mu \mathrm{s}]$ and $e=0.926$. Thus, the dissipation time-scale for contacts between two average sized particles, $t_{e}=2 m_{i j} /(\gamma)=$ $8.37[\mu \mathrm{s}]$ is considerably larger than $t_{c}$ and the background damping time-scale $t_{b}=\langle m\rangle / \gamma_{b}=$ $83.7[\mu \mathrm{s}]$ is much larger again, so that the particle- and contact-related time-scales are well separated. The strain-rate related timescale is $t_{s}=1 / \dot{\epsilon}_{z z}=0.1898[\mathrm{~s}]$. As usual in DEM, the integration time-step was chosen to be about 50 times smaller than the shortest time-scale $t_{c}$ [122].

Our numerical 'experiments' are performed in a three-dimensional triaxial box with periodic boundaries on all sides. One advantage of this configuration is the possibility of realizing different deformation modes with a single experimental set-up and a direct control of stress and/or strain [50, 128]. The systems are ideally homogeneous, which is assumed, but not tested in this study. The periodic walls can be strain-controlled to move following a cosinusoidal law such that, for example, the position of the top wall as function of time $t$ is 


$$
z(t)=z_{f}+\frac{z_{0}-z_{f}}{2}(1+\cos 2 \pi f t) \text { with strain } \epsilon_{z z}(t)=1-\frac{z(t)}{z_{0}},
$$

where $z_{0}$ is the initial box length and $z_{f}$ is the box length at maximum strain, respectively, and $f=T^{-1}$ is the frequency. The co-sinusoidal law allows for a smooth start-up and finish of the motion so that shocks and inertia effects are reduced. The maximum deformation is reached after half a period $t=T / 2$, and the maximum strain-rate applied during the deformation at $T / 4$ and $3 T / 4$ is $\dot{\epsilon}_{z z}^{\max }=2 \pi f\left(z_{0}-z_{f}\right) /\left(2 z_{0}\right)=\pi f\left(z_{0}-z_{f}\right) / z_{0}$.

Different strain-control modes are possible, like homogeneous strain-rate control for each time-step, applied to all particles and the periodic walls, i.e., the system boundaries or swelling instead of isotropic compression, as well as pressure-control of the (virtual) walls. However, this is not discussed, since it had no effect for the simple model used here, and for quasi-static deformations applied. For more realistic contact models and large strain-rates, the modes of strain- or stress-control have to be re-visited and carefully studied.

\subsection{Preparation and test procedure}

In this section, we describe first the sample preparation procedure and then the method for implementing the isotropic, uniaxial and deviatoric element test simulations. For convenience, the tensorial definitions of the different modes will be based on their respective strain-rate tensors. When presenting the numerical results, we will use the true strain as defined in section 2.4.2.1.

\subsubsection{Initial Isotropic preparation}

Since careful, well-defined sample preparation is essential in any physical experiment to obtain reproducible results [56], the preparation consists of three elements: (i) randomization, (ii) isotropic compression, and (iii) relaxation, all equally important to achieve the initial configurations for the following analysis. (i) The initial configuration is such that spherical particles are randomly generated in a 3D box, with a low volume fraction and rather large random velocities, such that they have sufficient space and time to move and to randomize themselves. (ii) This granular gas is then isotropically compressed in order to approach a isotropic configuration, to a target volume fraction $v_{0}=0.640$, this is sightly below the jamming volume fraction $v_{c} \approx 0.665$, i.e., the transition point from fluid-like behavior to solid-like behavior $[132,133,149]$. (iii) This is followed by a relaxation period at constant volume fraction to allow the particles to fully dissipate their energy and to achieve a static configuration in mechanical equilibrium.

Isotropic compression (negative strain-rate in our convention) can now be used to prepare further initial configurations at volume fractions $v_{i}$, after further relaxation, so that we have 
a series of different initial isotropic configurations, achieved during loading and unloading, as displayed in Fig. 2.1. The compression phase can be considered as the isotropic element test [69]. It is realized by a simultaneous inward movement of all the periodic boundaries of the system, with strain-rate tensor

$$
\dot{\mathbf{E}}=\dot{\varepsilon}_{\mathrm{v}}\left(\begin{array}{ccc}
-1 & 0 & 0 \\
0 & -1 & 0 \\
0 & 0 & -1
\end{array}\right)
$$

where $\dot{\varepsilon}_{\mathrm{v}}(>0)$ is the rate amplitude applied to the walls until the target volume fraction is achieved.

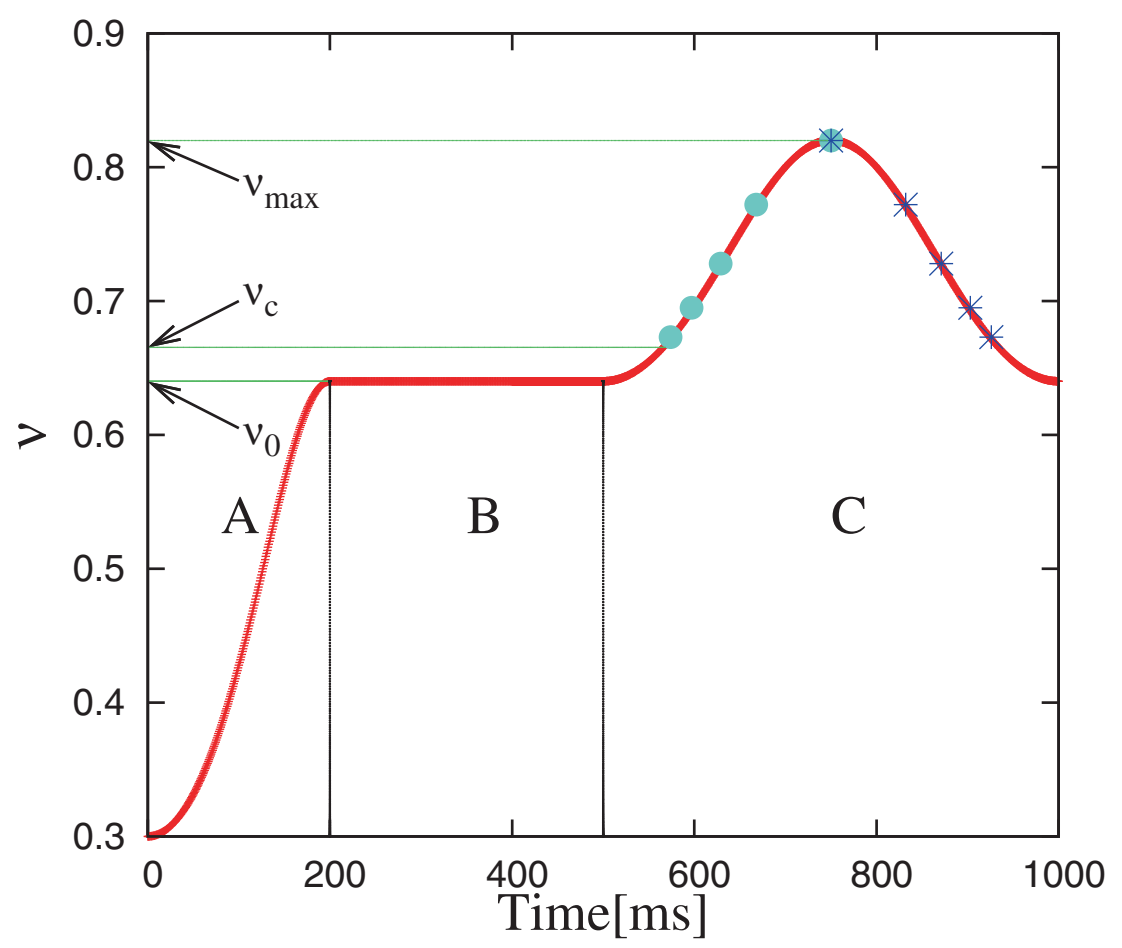

Figure 2.1: Evolution of volume fraction as a function of time. Region A represents the initial isotropic compression until the jamming volume fraction. B represents relaxation of the system and $\mathrm{C}$ represents the subsequent isotropic compression up to $v_{\max }=0.820$ and then decompression. Cyan dots represent some of the initial configurations, at different $v_{i}$, during the loading cycle and blue stars during the unloading cycle, which can be chosen for further study.

A schematic for implementing the isotropic, uniaxial and deviatoric deformation tests can be found in Ref. [84]. The procedure can be adapted for other non-volume conserving and/or stress-controlled modes (e.g., biaxial, triaxial and isobaric). One only has to use the same initial configuration and then decide which deformation mode to use, as shown in the figure under "other deformations". The corresponding schematic plots of deviatoric strain $\epsilon_{d}$ as a function of volumetric strain $\epsilon_{v}$ are shown below the respective modes. 


\subsubsection{Uniaxial}

Uniaxial compression is one of the element tests that can be initiated at the end of the "preparation", after sufficient relaxation indicated by the drop in potential energy due to particle overlaps to almost zero. The uniaxial compression mode in the triaxial box is achieved by a prescribed strain path in the $z$-direction, see Eq. 2.2, while the other boundaries $x$ and $y$ are non-mobile. During loading (compression) the volume fraction is increased, just as in the case of isotropic compression, from $v_{0}=0.64$ to a maximum volume fraction of $v_{\max }=0.820$ (as shown in region $\mathrm{C}$ of Fig. 2.1), and reverses back to the original volume fraction of $v_{0}$ during unloading. Uniaxial compression is defined by the strain-rate tensor

$$
\dot{\mathbf{E}}=\dot{\epsilon}_{\mathrm{u}}\left(\begin{array}{ccc}
0 & 0 & 0 \\
0 & 0 & 0 \\
0 & 0 & -1
\end{array}\right)
$$

where $\dot{\epsilon}_{\mathrm{u}}$ is the strain-rate (compression $>0$ and decompression/tension $<0$ ) amplitude applied in the uniaxial mode. The negative sign (convention) of $\dot{E}_{z z}$ corresponds to a reduction of length, so that tensile deformation is positive. Even though the strain is imposed only on the mobile "wall" in the $z$-direction, which leads to an increase of compressive stress on this wall during compression, the non-mobile walls also experience some stress increase due to the "push-back" stress transfer and rearrangement of the particles during loading, as discussed in more detail in the following sections. This is in agreement with theoretical expectations for materials with non-zero Poisson ratio. However, the stress on the passive walls is typically smaller than that of the mobile, active wall, as consistent with findings from laboratory element tests using the biaxial tester [141, 217] or the so-called $\lambda$-meter [108, 109].

\subsubsection{Deviatoric}

The preparation procedure, as described in section 2.3.1, provides different initial configurations with volume fractions $v_{i}$. For a deviatoric deformation element test, unless stated otherwise, the configurations are from the unloading part (represented by blue stars in Fig. 2.1 ), to test the dependence of quantities of interest on volume fraction, during volume conserving deviatoric (pure shear) deformations. The unloading branch is more reliable since it is much less sensitive to the protocol and rate of deformation during preparation [69]. Then, two different ways of deforming the system deviatorically are used, not to mention numberless superpositions of these. The deviatoric mode D2 has the strain-rate tensor

$$
\dot{\mathbf{E}}=\dot{\epsilon}_{\mathrm{D} 2}\left(\begin{array}{ccc}
1 & 0 & 0 \\
0 & 0 & 0 \\
0 & 0 & -1
\end{array}\right)
$$


where $\dot{\epsilon}_{\mathrm{D} 2}$ is the strain-rate (compression $>0$ ) amplitude applied to the wall with normal in $z$-direction. We use the nomenclature D2 since two walls are moving, while the third wall is stationary. The deviatoric mode D3 has the strain-rate tensor

$$
\dot{\mathbf{E}}=\dot{\epsilon}_{\mathrm{D} 3}\left(\begin{array}{ccc}
1 / 2 & 0 & 0 \\
0 & 1 / 2 & 0 \\
0 & 0 & -1
\end{array}\right)
$$

where $\dot{\epsilon}_{\mathrm{D} 3}$ is the $z$-direction strain-rate (compression $>0$ ) amplitude applied. In this case, D3 signifies that all the three walls are moving, with one wall twice as much (in opposite direction) as the other two, such that volume is conserved during deformation. Note that the D3 mode is uniquely similar in "shape" to the uniaxial mode, see Table 2.3 in the appendix, since in both cases two walls are controlled similarly. ${ }^{1}$

\subsection{Averaged quantities}

In this section, we present the general definitions of averaged microscopic and macroscopic quantities. The latter are quantities that are readily accessible from laboratory experiments, whereas the former are often impossible to measure in experiments but are easily available from discrete element simulations.

\subsubsection{Averaged microscopic quantities}

In this section, we define microscopic parameters including the coordination number, the fraction of rattlers, and the ratio of the kinetic and potential energy.

\subsubsection{Coordination number and fraction of rattlers}

In order to link the macroscopic load carried by the sample with the microscopic contact network, all particles that do not contribute to the force network - particles with exactly zero contacts - are excluded. In addition to these "rattlers" with zero contacts, there may be a few particles with some finite number of contacts, for some short time, which thus also do not contribute to the mechanical stability of the packing. These particles are called dynamic rattlers [69], since their contacts are transient: The repulsive contact forces will push them away from the mechanically stable backbone [69]. Frictionless particles with less than 4 contacts are rattlers, since they cannot be mechanically stable and hence do not

1. The more general, objective definition of deviatoric deformations is to use the orientation of the stresses (eigen-directions) in the deviatoric plane from the eigenvalues, as explored elsewhere [196], since this is beyond the scope of this study. Mode D2 is different in this respect and thus resembles more an independent mode, so that we plot by default the D2 results rather than the D3 ones. The mode D2, with shape factor $\zeta=0$, is on the one hand similar to the simple-shear situation, and on the other hand allows for simulation of the biaxial experiment (with two walls static, while four walls are moving [141, 217]). 
contribute to the contact network [69]. In this work, since tangential forces are neglected, rattlers can thus be identified by just counting their number of contacts. This leads to the following abbreviations and definitions for the coordination number (i.e. the average number of contacts per particle) and fraction of rattlers, which must be re-considered for systems with tangential and other forces or torques:

$$
\begin{aligned}
& N: \text { Total number of particles. } \\
& N_{4}:=N_{C \geq 4}: \text { Number of particles with at least } 4 \text { contacts. } \\
& M: \text { Total number of contacts } \\
& M_{4}:=M_{C \geq 4}: \text { Total number of contacts of particles with at least } 4 \text { contacts. } \\
& C^{r}:=\frac{M}{N} \quad: \text { Coordination number (simple definition). } \\
& C:=C^{m}=\frac{M_{4}}{N} \quad: \text { Coordination number (modified definition). } \\
& C^{*}:=\frac{M_{4}}{N_{4}}=\frac{C}{1-\phi_{r}} \quad: \text { Corrected coordination number. } \\
& \phi_{r}:=\frac{N-N_{4}}{N} \quad: \text { (Number) fraction of the rattlers. } \\
& v:=\frac{1}{V} \sum_{p \in N} V_{p}: \text { Volume fraction of the particles. }
\end{aligned}
$$

Some simulations results for the coordination numbers and the fraction of rattlers will be presented below, in subsection 2.5.1.

\subsubsection{Energy ratio and the Quasi-Static Criterion}

Above the jamming volume fraction $v_{c}$, in mechanically stable static situations, there exist permanent contacts between particles, hence the potential energy (which is also an indicator of the overlap between particles) is considerably larger than the kinetic energy (which has to be seen as a perturbation).

The ratio of kinetic energy and potential energy is shown in Fig. 2.2 for isotropic compression from $v_{1}=0.673$ to $v_{\max }=0.820$ and back. The first simulation, represented by the solid red line, was run for a simulation time $T=5000 \mu$ s and the second (much slower) simulation, represented by the green dashed line was run for $T=50000 \mu \mathrm{s}$. For these, the maximum strain-rates are $\dot{\epsilon}_{z z}^{\max }=52.68\left[\mathrm{~s}^{-1}\right]$ and $5.268\left[\mathrm{~s}^{-1}\right]$, respectively. During compression, with increasing volume fraction, the energy ratio generally decreases and slower deformation by a factor of 10 leads to more than 100 times smaller energy ratios with stronger fluctuations. Most sharp increase of the energy ratio represents re-organization events of several particles and are followed by an exponentially fast decrease (data not shown). The decrease is controlled by the interaction and dissipation time-scales and not by the shear rate; only due to the scaling of $t_{s}$, the decrease appears to be faster for the slower deformation. More explic- 
itly, the rate of decay depends on material parameters only and is of the order of $1 / t_{e}$. The low initial ratio of kinetic to potential energy $\left(E_{k} / E_{p}<0.001\right)$ indicates that the system is in the jammed regime and is almost in the quasi-static state. To ensure that the quasi-static criterion is fulfilled in the simulations performed for the various deformation modes, all the simulations are run at a very small strain-rate. In this way, dynamic effects are minimized and the system is as close as feasible to the quasi-static state. For many situations, it was tested that a slower deformation did not lead to considerably different results. For the majority of the data presented, we have $E_{k} / E_{p} \leq 10^{-3}$. Lower energy ratios can be obtained by performing simulations at even slower rates but the settings used are a compromise between computing time and reasonably slow deformations.

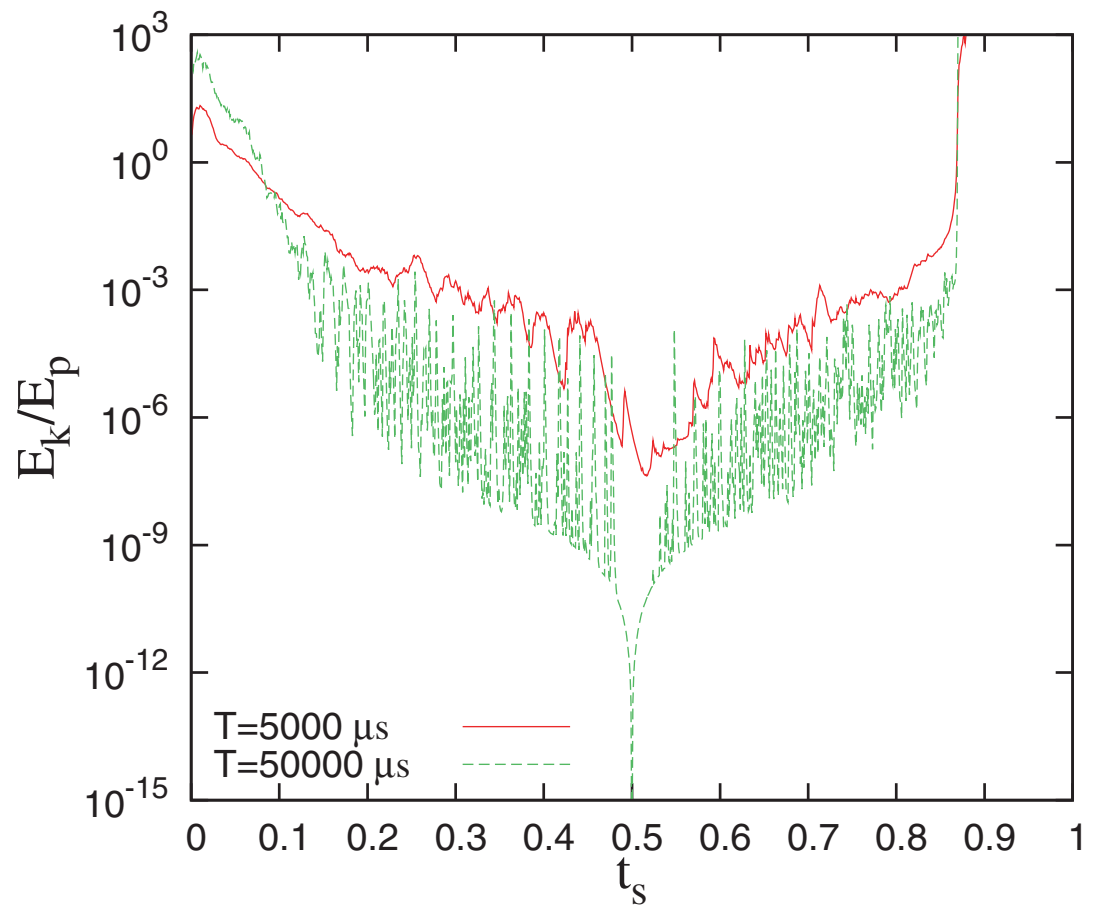

Figure 2.2: Comparison of the ratio of kinetic and potential energy in scaled time $\left(t_{s}=t / T\right)$ for two simulations, with different period of one compression-decompression cycle $T$, as given in the inset.

\subsubsection{Averaged macroscopic quantities}

Now the focus is on defining averaged macroscopic tensorial quantities - including strain, stress- and fabric (structure) tensors - that reveal interesting bulk features and provide information about the state of the packing due to its deformation. 


\subsubsection{Strain}

For any deformation, the isotropic part of the infinitesimal strain tensor $\varepsilon_{\mathrm{v}}$ is defined as:

$$
\varepsilon_{\mathrm{v}}=\dot{\varepsilon}_{\mathrm{v}} \mathrm{dt}=\frac{\epsilon_{x x}+\epsilon_{y y}+\epsilon_{z z}}{3}=\frac{1}{3} \operatorname{tr}(\mathbf{E})=\frac{1}{3} \operatorname{tr}(\dot{\mathbf{E}}) \mathrm{dt},
$$

where $\varepsilon_{\alpha \alpha}=\dot{\varepsilon}_{\alpha \alpha} \mathrm{dt}$ with $\alpha \alpha=x x, y y$ and $z z$ as the diagonal elements of the strain tensor $\mathbf{E}$ in the Cartesian $x, y, z$ reference system. The trace integral of $3 \varepsilon_{\mathrm{v}}$ denoted by $3 \varepsilon_{v}$, is the true or logarithmic strain, i.e., the volume change of the system, relative to the initial reference volume, $V_{0}$ [69].

Several definitions are available in literature $[83,196,223]$ to define the deviatoric magnitude of the strain. For the sake of simplicity, we use the following definition of the deviatoric strain to account for all active and inactive directions in a triaxial experiment, regardless of the deformation mode,

$$
\varepsilon_{\mathrm{dev}}=\sqrt{\frac{\left(\epsilon_{x x}-\epsilon_{y y}\right)^{2}+\left(\epsilon_{y y}-\epsilon_{z z}\right)^{2}+\left(\epsilon_{z z}-\epsilon_{x x}\right)^{2}}{2}},
$$

since, for our triaxial box, for all modes, the Cartesian coordinates resemble the eigensystem, with eigenvalues sorted according to magnitude $\epsilon_{\mathrm{d}}{ }^{(1)} \geq \epsilon_{\mathrm{d}}{ }^{(2)} \geq \epsilon_{\mathrm{d}}{ }^{(3)}$, which leaves the eigenvalue $\epsilon_{\mathrm{d}}{ }^{(1)}$ as the maximal tensile eigenvalue, with corresponding eigen-direction, and $\varepsilon_{\mathrm{dev}} \geq 0$ as the magnitude of the deviatoric strain. ${ }^{2}$ The description of the tensor is completed by either its third invariant or, equivalently, by the shape factor $\zeta$, as given in Table 2.3 in the appendix. Note that the values for $\zeta$ are during uniaxial loading, where compression is performed in the $z$-direction. The sorting will lead to different values, $\zeta=-1 / 2$, after the strain is reversed for both UNI and D3 modes.

\subsubsection{Stress}

From the simulations, one can determine the stress tensor (compressive stress is positive as convention) components:

$$
\sigma_{\alpha \beta}=\frac{1}{V}\left(\sum_{p \in V} m^{p} v_{\alpha}^{p} v_{\beta}^{p}-\sum_{c \in V} f_{\alpha}^{c} l_{\beta}^{c}\right),
$$

with particle $p$, mass $m^{p}$, velocity $v^{p}$, contact $c$, force $f^{c}$ and branch vector $l^{c}$, while Greek letters represent components $x, y$, and $z[123,124]$. The first sum is the kinetic energy tensor and the second involves the contact-force dyadic product with the branch vector. Averaging, smoothing or coarse graining [212] in the vicinity of the averaging volume, $V$, weighted

2. The objective definition of the deviatoric strain defines it in terms of the eigenvalues $\epsilon_{\mathrm{d}}{ }^{(1)}, \epsilon_{\mathrm{d}}{ }^{(2)}$ and $\epsilon_{\mathrm{d}}{ }^{(3)}$, of the (deviatoric) tensor. However, since the global strain is given by the wall motion, the two definitions are equivalent for triaxial element tests. 
according to the vicinity is not applied in this study, since averages are taken over the total volume. Furthermore, since the data in this study are quasi-static, the first term can be neglected.

The average isotropic stress (i.e. the hydrostatic pressure) is defined as:

$$
P=\frac{\sigma_{x x}+\sigma_{y y}+\sigma_{z z}}{3}=\frac{1}{3} \operatorname{tr}(\sigma),
$$

where $\sigma_{x x}, \sigma_{y y}$ and $\sigma_{z z}$ are the diagonal elements of the stress tensor in the $x, y$ and $z$ boxreference system and $\operatorname{tr}(\sigma)$ is its trace. The non-dimensional pressure [69] is defined as:

$$
p=\frac{2\langle r\rangle}{3 k} \operatorname{tr}(\sigma),
$$

where $\langle r\rangle$ is the mean radius of the spheres and $k$ is the contact stiffness defined in section 2.2 .

We define the deviatoric magnitude of stress (similar to Eq. (2.4) for deviatoric strain) as:

$$
\sigma_{\mathrm{dev}}=\sqrt{\frac{\left(\sigma_{x x}-\sigma_{y y}\right)^{2}+\left(\sigma_{y y}-\sigma_{z z}\right)^{2}+\left(\sigma_{z z}-\sigma_{x x}\right)^{2}}{2}},
$$

which is always positive by definition. The direction of the deviatoric stress is carried by its eigen-directions, where stress eigenvalues are sorted like strain eigenvalues according to their magnitude. Eqs. (2.4) and (2.8) can easily be generalized to account for shear reversal using a sign convention taken from the orientation of the corresponding eigenvectors, or from the shape-factor, however, this will not be detailed here for the sake of brevity.

It is noteworthy to add that the definitions of the deviatoric stress and strain tensors are proportional to the second invariants of these tensors, e.g., for stress: $\sigma_{\mathrm{dev}}=\sqrt{3 J_{2}}$, which makes our definition. identical to the von Mises yield criterion $[60,196]^{3}$

\subsubsection{Fabric (structure) tensor}

Besides the stress of a static packing of powders and grains, the next most important quantity of interest is the fabric/structure tensor. The expression for the components of the fabric tensor is:

$$
F_{\alpha \beta}=\left\langle F^{p}\right\rangle=\frac{1}{V} \sum_{p \in V} V^{p} \sum_{c=1}^{N} n_{\alpha}^{c} n_{\beta}^{c},
$$

where $V^{p}$ is the particle volume which lies inside the averaging volume $V$, and $n^{c}$ is the normal vector pointing from the center of particle $p$ to contact $c . F_{\alpha \beta}$ are thus the components

3. Different factors in the denominator of Eqs. (2.4) and (2.8) have been proposed in literature [83, 223] but they only result in a change in the maximum deviatoric value obtained. For consistency, we use the same factor $\sqrt{1 / 2}$ for deviatoric stress and strain and a similar definition for the deviatoric fabric, see the next subsection. 
of a symmetric rank two $3 \times 3$ tensor like the stress tensor. The isotropic fabric, $F_{\mathrm{v}}=\operatorname{tr}(\mathbf{F}) / 3$, quantifies the contact number density as studied in Ref. [69]. We assume that the structural anisotropy in the system is quantified (completely) by the anisotropy of fabric, i.e., the deviatoric fabric. To quantify it, we define a scalar similar to Eqs. (2.4) and (2.8) as:

$$
F_{\mathrm{dev}}=\sqrt{\frac{\left(F_{x x}-F_{y y}\right)^{2}+\left(F_{y y}-F_{z z}\right)^{2}+\left(F_{z z}-F_{x x}\right)^{2}}{2}},
$$

where $F_{x x}, F_{y y}$ and $F_{z z}$ are the three diagonal components of the fabric tensor. The fabric tensor practically has only diagonal components with non-diagonal elements very close to zero, so that its eigen system is close to the Cartesian, as confirmed by eigen system analysis.

\subsubsection{Conclusion}

Three macroscopic rank-two tensors were defined and will be related to microscopic quantities and each other in the following. The orientations of all the tensor eigenvectors show a tiny non-colinearity of stress, strain and fabric, which we neglect in the next sections, since we attribute it to natural statistical fluctuations. Furthermore, the shape factor defined for strain can also be analyzed for stress and fabric, as will be shown elsewhere.

\subsection{Evolution of micro-quantities}

In this section, we discuss the evolution of the microscopic quantities studied - including coordination number and fraction of rattlers - as function of volume fraction and deviatoric strain respectively, and compare these results for the different deformation modes.

\subsubsection{Coordination number and fraction of rattlers}

It has been observed [69] that under isotropic deformation, the corrected coordination number $C^{*}$ follows the power law

$$
C^{*}(v)=C_{0}+C_{1}\left(\frac{v}{v_{c}}-1\right)^{\alpha}
$$

where $C_{0}=6$ is the isostatic value of $C^{*}$ in the frictionless case. For the uniaxial unloading simulations, we obtain $C_{1} \approx 8.370, \alpha \approx 0.5998$ and $v_{c}^{\mathrm{UNI}} \approx 0.6625$ as best fit parameters.

In Fig. 2.3, the evolution of the simple, corrected and modified coordination numbers are compared as functions of volume fraction during uniaxial deformation (during one loading and unloading cycle). The compression and decompression branches are indicated by arrows pointing right and left, respectively. The contribution to the contact number originating from particles with $C=1,2$ or 3 is small - as compared to those with $C=0-$ since $C^{r}$ and $C^{m}$ are 


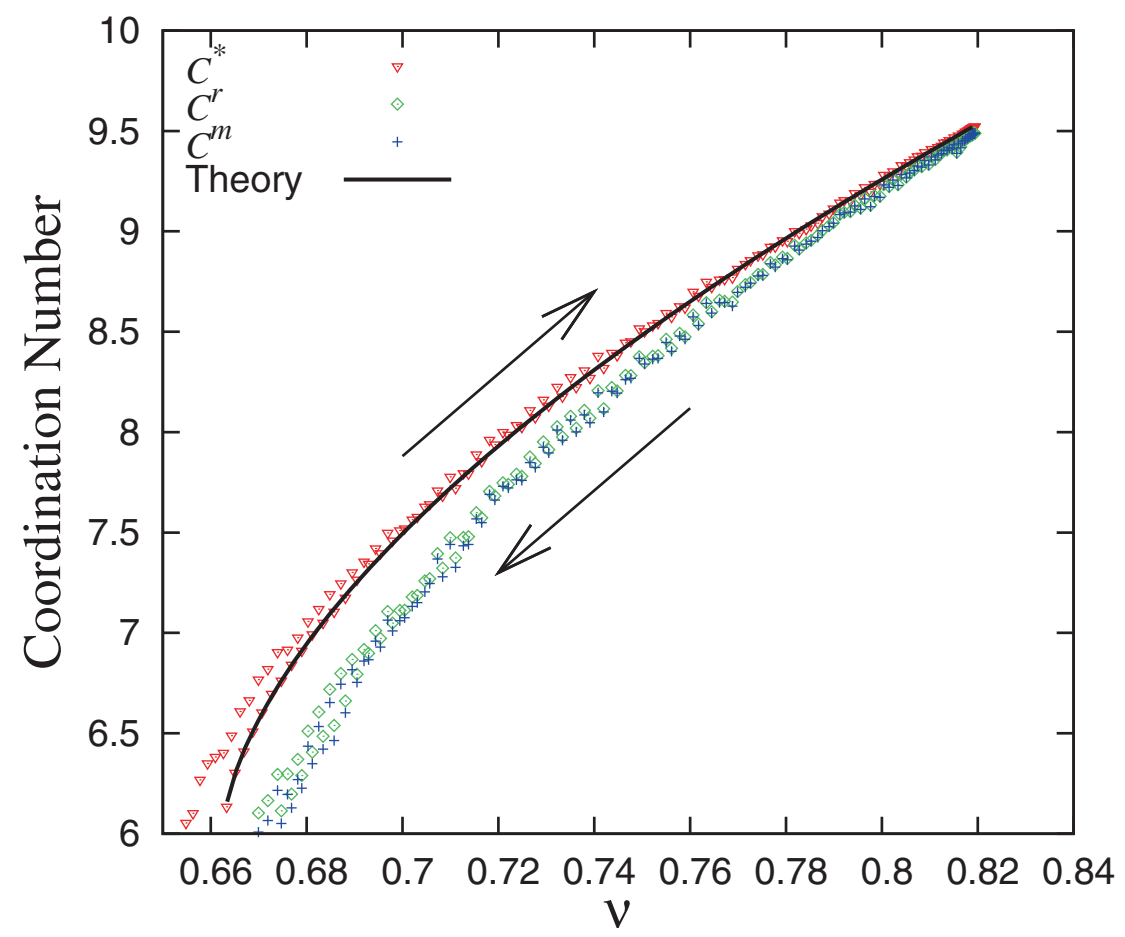

Figure 2.3: Comparison between coordination numbers using the simple ('+', blue), modified (' $\diamond$ ', green) and corrected (' $\boldsymbol{\nabla}$ ', red) definitions. Data are from a uniaxial compressiondecompression simulation starting from $v_{0}=0.64<v_{c} \approx 0.6625$. The solid black line represents Eq. (2.11), with parameters given in the text, very similar to those measured in Ref. [69], see Table 2.1.

very similar, but always smaller than $C^{*}$, due to the fraction of rattlers, as discussed below. The number of contacts per particle grows with increasing compression to a value of $C^{*} \approx$ 9.5 at maximum compression. During decompression, the contacts begin to open and the coordination number decreases and approaches the theoretical value $C_{0}=6$ at the critical jamming volume fraction after uniaxial de-compression $v_{c}^{\mathrm{UNI}} \approx 0.662$. Note that the $v_{c}^{\mathrm{UNI}}$ value is smaller than $v_{c}^{\text {ISO }} \approx 0.665$ reached after purely isotropic over-compression to the same maximal volume fraction. ${ }^{4}$ The coordination numbers are typically slightly larger in the loading branch than in the unloading branch, due to the previous over-compression.

In Fig. 2.4, we plot the corrected coordination number for deformation mode D2 as a function of the deviatoric strain for five different volume fractions. Two sets of data are presented for each volume fraction starting from different initial configurations, either from the loading or the unloading branch of the isotropic preparation simulation (cyan dots and blue stars in Fig. 2.1). Given initial states with volume fractions above the jamming volume fraction,

4. The value, $C_{0}=6$, is expected since it is the isostatic limit for frictionless systems in three dimensions [69], for which the number of constraints (contacts) is twice the number of degrees of freedom (dimension) - in average, per particle - so that the number of unknown forces matches exactly the number of equations. $\left(C_{0}\right.$ is different from the minimal number for a mechanically stable sphere $C_{\min }=4$ in $3 \mathrm{D}$ ). 


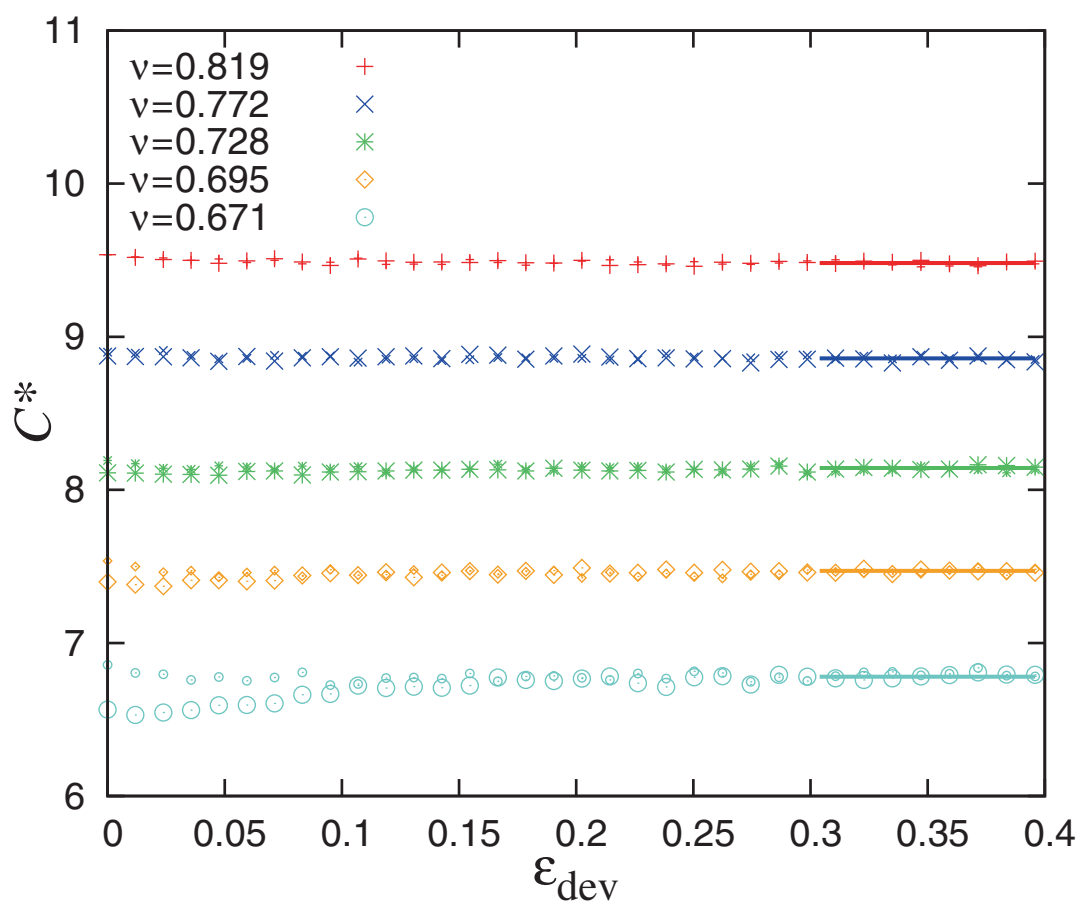

Figure 2.4: Evolution of coordination number with deviatoric strain for the D2 mode. Smaller symbols represent data with initial configuration from the loading branch of an isotropic simulation, while the larger symbols start from an initial configuration with the same volume fraction, but from the isotropic unloading branch. The horizontal line at the large strain of the dataset indicates an average after saturation at steady state.

and due to the volume conserving D2 mode, the value of the coordination number remains practically constant. Only for the lowest volume fractions, close to jamming, a slight increase (decrease) in $C^{*}$ can be seen, for initial states chosen from the unloading (loading) branch of the preparation step. However, both reach similar steady-state values after large strain, as indicated by the solid lines. Hence, for further analysis, unless otherwise stated, we will only present the steady-state values of micro- and macro-quantities from deviatoric modes D2 and D3.

The re-arrangement of the particles during shear thus does not lead to the creation (or destruction) of many contacts - on average. There is no evidence of a change in the average number of contacts after $10-15$ percent of strain. However, close to jamming, a clear dependence of $C^{*}$ on the initial state exists, which vanishes in steady state when one gets saturated values in micro- and macro-quantities, after large enough strain. For the same volume fraction, we evidence a range of $C_{o c}^{*} \leq C_{s}^{*} \approx C_{i c}^{*}$, where the subscripts refer to overcompressed, steady, and initially compressed states, respectively. The coordination number (or alternatively the contact number density, as related to the trace of the fabric tensor) is thus is a control parameter closely linked to the volume fraction that contains more information about the structure than $v$ (above the jamming volume fraction), see Refs. $[110,111]$ and 
references therein.

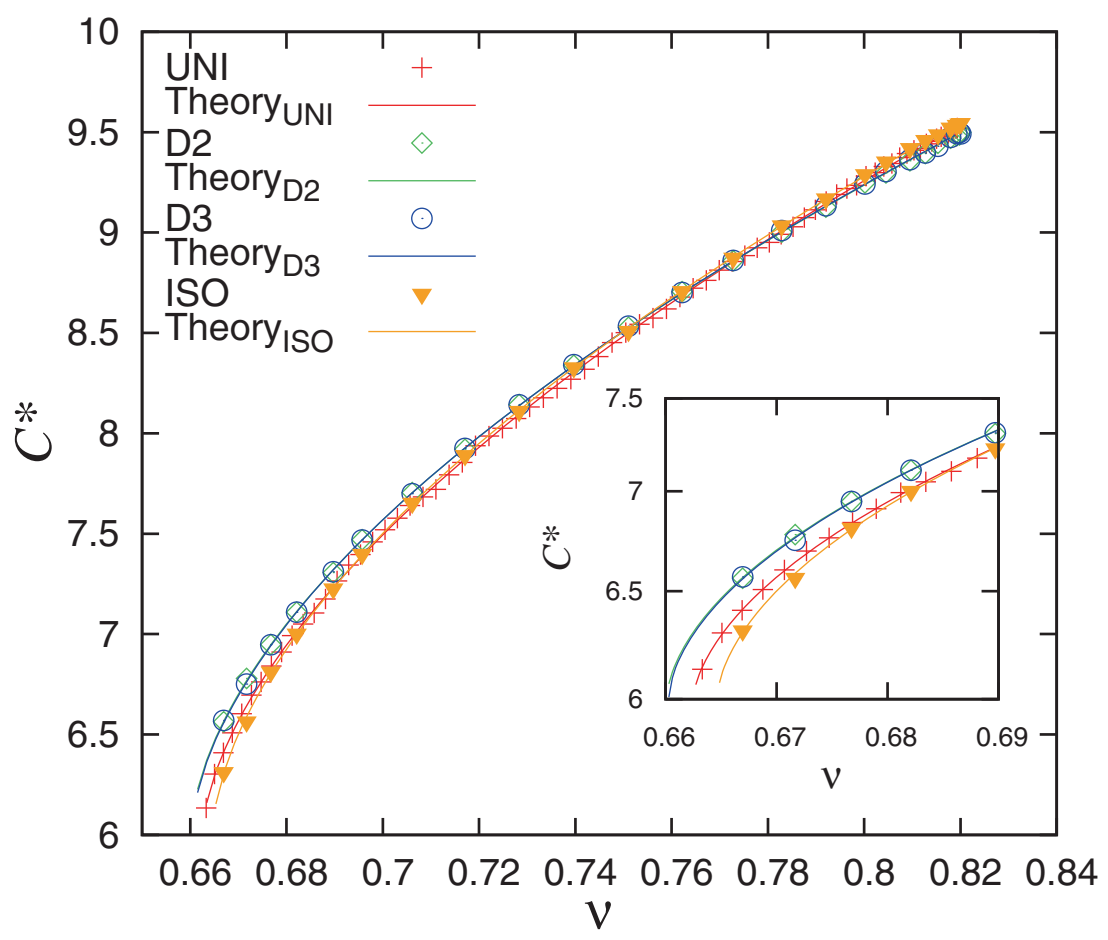

Figure 2.5: Evolution of the corrected coordination number as a function of volume fraction during unloading for all modes. The symbols represent the respective simulation data while the solid lines represent the analytical equation according to Eq. (2.11) with the respective values of $C_{0}, C_{1}, \alpha$ and $v_{c}$ shown in Table 2.1. Inset shows the corrected coordination number at lower volume fractions closer to jamming.

In Fig. 2.5, the corrected coordination number is shown as a function of volume fraction for the purely isotropic unloading, the uniaxial unloading, and the large strain deviatoric deformation datasets. Different symbols show the values of $C^{*}$ for the different deformation modes for various volume fractions. Interestingly, the power law for the coordination number, derived from isotropic data, describes well also the uniaxial and deviatoric data, with coefficients given in Table 2.1. This suggests that (for the cases considered), when particles are frictionless, the coordination number is independent on the deviatoric strain, in steady state, and the limit values can be approximated by Eq. (2.11), as proposed for simple isotropic deformation. The distinction between the modes at the small (isotropic) strain region is shown in the inset of Fig. 2.5. The mixed mode (uniaxial) is bounded on both sides by the pure modes, namely isotropic and deviatoric (D2 and D3 cannot be distinguished), indicating that the two pure modes are limit states or extrema for $C^{*}$. Alternatively, the range in $C^{*}$ values can be seen as caused by a range in $v_{c}$, with $v_{c}^{\mathrm{ISO}}>v_{c}^{\mathrm{UNI}}>v_{c}^{\mathrm{DEV}}$, which represent the maximal jamming volume fraction after previous (isotropic, strong) over-compression, the intermediate jamming volume fraction after (mixed mode) deformation, and the minimal jamming volume fraction after large deviatoric strain, respectively, with $v_{c}^{\text {ISO }} \approx 0.6646$ 


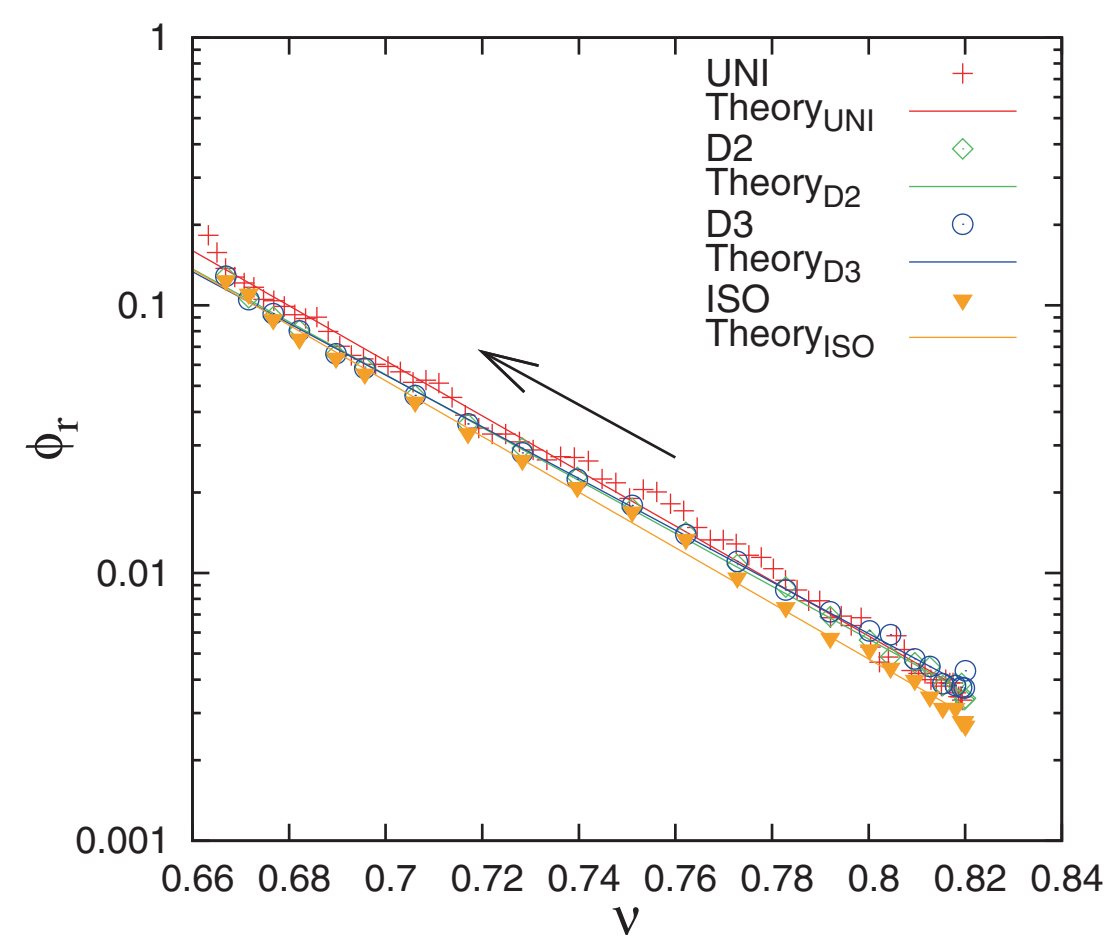

Figure 2.6: Evolution of the fraction of rattlers as a function of volume fraction during unloading for all modes. The symbols represent the respective simulation data. The solid lines are the analytical fits of Eq. (2.12) for each mode with the values of fit parameters $\phi_{c}$ and $\phi_{v}$ for each mode shown in Table 2.1. The arrow indicates the unloading direction.

and $v_{c}^{\mathrm{DEV}} \approx 0.6602$. In other words, deviatoric deformations reduce the jamming volume fraction of the packing, i.e, can disturb and dilate a dense (over-compressed) packing so that it becomes less efficiently packed. This is opposite to isotropic over-compression, where after unloading, the jamming volume fraction is higher, i.e., the system is more efficiently packed/structured. This behavior is qualitatively to be expected for frictional particles, however, this is to our knowledge the first time that this small but systematic range of jamming volume fractions is reported for frictionless packings - where the most relevant and only mechanism is structural reorganization, as will be discussed further in section 2.6.1.1.

As related interesting microscopic quantity, we recall the analytical expression for the fraction of rattlers proposed in Ref. [69]:

$$
\phi_{r}(v)=\phi_{c} \exp \left[-\phi_{v}\left(\frac{v}{v_{c}}-1\right)\right],
$$

where the best-fit parameters for the different deformation modes are given in Table 2.1, and $v_{c} \approx 0.6646$ is obtained from extrapolation of $C^{*}$ to the isostatic coordination number $C_{0}=6$. In Fig. 2.6, the evolution of the fraction of rattlers is plotted as a function of volume fraction for both isotropic and uniaxial unloading as well as for steady state deviatoric mode simulations. We then compare these with the prediction/fit (solid lines) from the exponential 


\begin{tabular}{cccc}
\hline \hline$C^{*}$ & $C_{1}$ & $\alpha$ & $v_{c}$ \\
\hline ISO [69] & $8 \pm 0.5$ & $0.58 \pm 0.05$ & $0.66 \pm 0.01$ \\
ISO & 8.2720 & 0.5814 & 0.6646 \\
UNI & 8.370 & 0.5998 & 0.6625 \\
D2 & 7.9219 & 0.5769 & 0.6601 \\
D3 & 7.9289 & 0.5764 & 0.6603 \\
\hline$\phi_{r}$ & $\phi_{c}$ & $\phi_{v}$ & \\
\hline ISO [69] & $0.13 \pm 0.03$ & $15 \pm 2$ & \\
ISO & 0.1216 & 15.8950 & \\
UNI & 0.1507 & 15.6835 & \\
D2 & 0.1363 & 15.0010 & \\
D3 & 0.1327 & 14.6813 & \\
\hline$p^{*}$ & $p_{0}$ & $\gamma_{p}$ & $v_{c}$ \\
\hline ISO [69] & 0.0418 & 0.110 & 0.666 \\
ISO & 0.04172 & 0.06228 & 0.6649 \\
UNI & 0.04006 & 0.03270 & 0.6619 \\
D2 & 0.03886 & 0.03219 & 0.6581 \\
D3 & 0.03899 & 0.02893 & 0.6583 \\
\hline
\end{tabular}

Table 2.1: Fit parameters for the analytical predictions of coordination number, fraction of rattlers, and pressure in Eqs. (2.11) with $C_{0}=6$, (2.12) and (2.14), respectively. For the $\phi_{r}$ fits, $v_{c}$ is used from the $C^{*}$ fits for the different deformation modes. The first rows of isotropic data are from Ref. [69], for various polydispersities and also during unloading, but for different over-compression.

decay equation, Eq. (2.12). Interestingly, in contrast to the coordination number, the fraction of rattlers displays strongest differences at the highest volume fraction $(v=0.82$ in Fig. 2.6), and it is lowest during isotropic unloading, as compared to the steady state deviatoric mode situations, and somewhat higher during uniaxial unloading. The difference between the modes is smallest close to jamming. For uniaxial simulations, at the end of unloading, close to $v_{c}^{\mathrm{UNI}}$, a considerable fraction (almost 20 percent) of the total number of particles are rattlers that do not contribute to the stability of the network. For higher volume fractions, a strong exponential decay is evidenced. ${ }^{5}$

5. The sharp jump observed in Ref. [69] at the jamming transition during unloading is not seen here because the system remains above the jammed state. Interestingly, the simulation data for the uniaxial and deviatoric mode all collapse close to the (isotropic) exponential prediction. 


\subsection{Evolution of macro-quantities}

In this section, we discuss results for the evolution of the macroscopic tensor quantities stress and fabric, as defined in section 2.4.2. For clarity, we split them in isotropic and deviatoric parts in subsections 2.6.1 and 2.6.2, respectively.

\subsubsection{Evolution of macro-quantities: Isotropic part}

Here, we briefly discuss the isotropic pressure and isotropic fabric, for different deformations modes, as functions of volume fraction and/or isotropic strain.

\subsubsection{Isotropic pressure}

In this section, the relation between pressure and volume fraction is studied. First, we consider the contact deformations, since the force is related to the contact overlap/deformation $\delta_{c}$, and stress is proportional to the force. The infinitesimal change $\mathrm{d}\langle\Delta\rangle_{c}=3 D \epsilon_{\mathrm{v}}$, of the normalized average overlap, $\Delta_{c}=\delta_{c} /\langle r\rangle$, can be related to the volumetric strain under the simplifying assumption of uniform, homogeneous deformation in the packing. As defined in subsection 2.4.2.1, $\epsilon_{\mathrm{v}}=\epsilon_{i i} / 3$ is the trace of the infinitesimal strain tensor increment, and $D \approx 0.425$ is a proportionality constant that depends on the particle size distribution and can be readily obtained from the average overlap and volume fraction (data not shown), see Eq. (2.13). The integral of $\epsilon_{\mathrm{V}}$, denoted by $\varepsilon_{v}$, is the true or logarithmic volume change of the system, relative to the reference volume $V_{\text {ref }}$. This is chosen, without loss of generality, at the critical jamming volume fraction $v_{\text {ref }}=v_{c}$, so that the average normalized overlap is [69]

$$
\langle\Delta\rangle_{c}=-D \int_{V_{0}}^{V} \epsilon_{\mathrm{v}}=-D \varepsilon_{\mathrm{v}}=D \ln \left(\frac{v}{v_{c}}\right)
$$

As in Eq. (2.7), see Refs. [69, 179] for details, the non-dimensional pressure is:

$$
p=\frac{2\langle r\rangle}{3 k} \operatorname{tr}(\sigma)=p_{0} \frac{v C}{v_{c}}\left(-\varepsilon_{\mathrm{v}}\right)\left[1-\gamma_{p}\left(-\varepsilon_{\mathrm{v}}\right)\right],
$$

and the scaled pressure is:

$$
p^{*}=\frac{p v_{c}}{v C}=p_{0}\left(-\varepsilon_{\mathrm{v}}\right)\left[1-\gamma_{p}\left(-\varepsilon_{\mathrm{v}}\right)\right]
$$

where $p_{0} \approx 0.04006, \gamma_{p} \approx 0.03270$, and the critical volume fraction $v_{c} \approx 0.6619$ are fit parameters to pressure for uniaxial unloading. Combining the quasi-static parts of Eqs. (2.5) and (2.14), leads to the proportionality $p \propto v C \Delta_{c}$, which makes the $p$ a measure for the average overlap relative to the average particle diameter, and $p^{*}$ scales all $p$ on the same reference volume fraction, i.e., the jamming volume fraction. 
Note that the critical volume fraction $v_{c} \approx 0.6625$ obtained from extrapolation of $C^{*}$ to the isostatic coordination number $C_{0}=6$ is very close to that obtained from Eq. (2.14). When fitting all modes with pressure, one confirms again that $v_{c}^{\mathrm{UNI}}$ falls in between the pure modes $\left(v_{c}^{\mathrm{ISO}}\right.$ and $\left.v_{c}^{\mathrm{DEV}}\right)$, with all fits quite consistent within each mode, as summarized in Table 2.1.

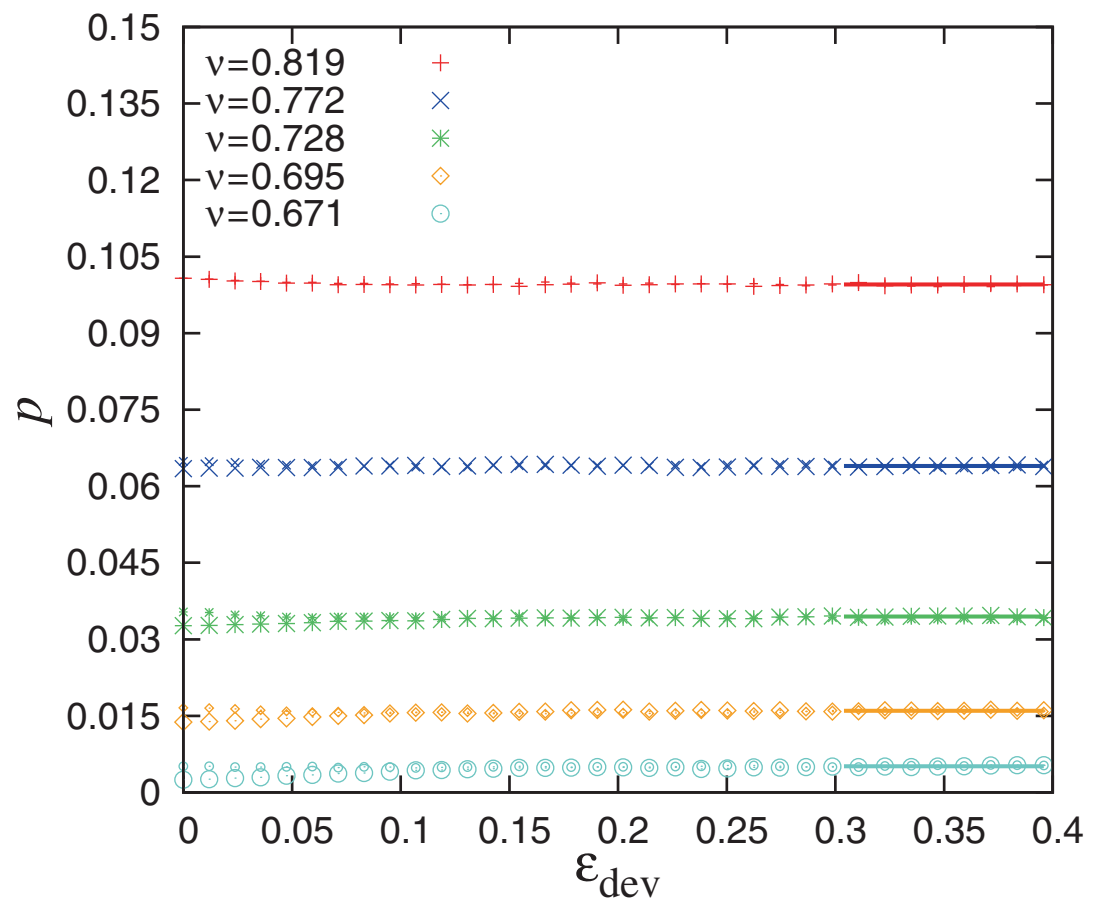

Figure 2.7: Evolution of (non-dimensional) pressure, Eq. (2.7), with deviatoric strain for the D2 deformation mode, at different initial volume fractions $v_{i}$. Small and large symbols represent simulations starting with initial isotropic configurations from the loading and unloading branch, respectively. The horizontal line at the large strain of the dataset indicates an average value of the pressure after saturation at steady state.

In Fig. 2.7, we plot the total (non-dimensional) pressure $p$ for deformation mode D2 as a function of the deviatoric strain for different volume fractions. Above the jamming volume fraction, the value of the pressure remains practically constant except for the lowest volume fractions close to jamming where a slight increase in $p$ can be seen when initial states are chosen from the unloading branch of isotropic modes (blue stars in Fig. 2.1), whereas a slight decrease in $p$ is observed for initial states chosen from the loading branch (cyan dots in Fig. 2.1). Independent of the initial configuration, pressure reaches the same steady state value at larger strains, very similar to the behavior of the coordination number.

Fig. 2.8 shows the total pressure as a function of the volume fraction for isotropic and uniaxial unloading. The deviatoric mode D2 and D3 data, are obtained after large deviatoric strain, as shown for D2 in Fig. 2.7. The pressure increases non-linearly during loading (data not shown) until the predetermined maximum volume fraction is reached [69], and also during unloading, the pressure decays non-linearly. Due to the linear contact model, 


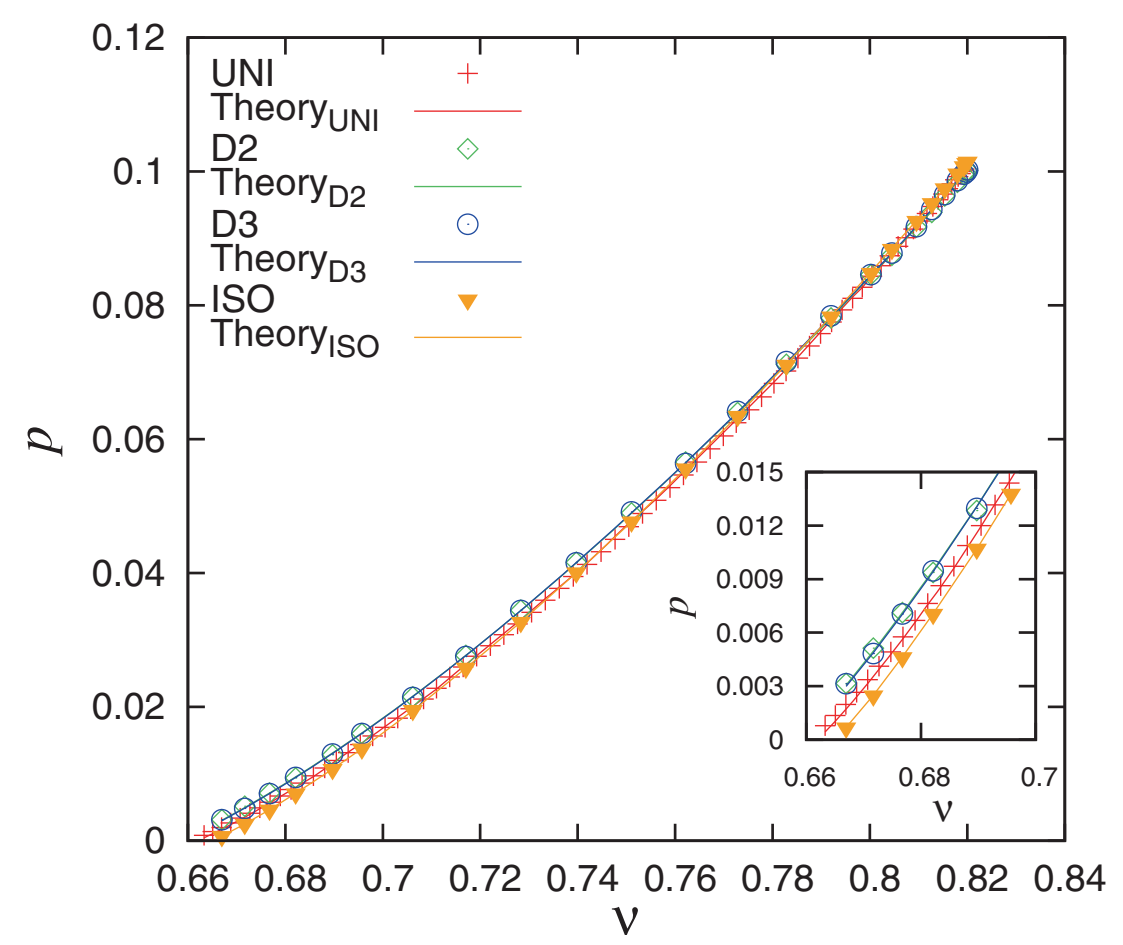

Figure 2.8: Total (non-dimensional) pressure, Eq. (2.14), plotted as function of volume fraction for the uniaxial and isotropic datasets during unloading, and for the D2/D3 deviatoric modes after large strain. The solid lines are the analytical fits of Eq. (2.14), for each mode, with parameters $p_{0}, \gamma_{p}$ and $v_{c}$ shown in Table 2.1 .

this can be directly related to contact number density, i.e., the isotropic fabric, which quantifies the isotropic, direction independent changes of structure due to re-arrangements and closing/opening of contacts.

In Fig. 2.9, we plot the scaled pressure defined in Eq. (2.15) against the volumetric strain from the same data as in Fig. 2.8. The three datasets almost collapse for small strain. For increasing volume fractions (larger $-\varepsilon_{\mathrm{v}}$ ) the isotropic mode scaled pressure is considerably larger than the uniaxial and the deviatoric datasets, where again the uniaxial data fall in between isotropic and deviatoric data sets. This is consistent with the fact that the uniaxial mode is a superposition of the purely isotropic and deviatoric deformation modes and resembles very much the behavior of $C^{*}$.

The dependence of pressure on isotropic strain can be interpreted in relation to sample history. The deviatoric modes (D2 or D3) lead to dilatancy and thus to the highest steady state pressure, with respectively lowest $v_{c}^{\mathrm{DEV}}$; the isotropic mode is strictly compressive, with the lowest pressure, after over-compression, during unloading, with respectively the highest $v_{c}^{\text {ISO }}$; and the uniaxial mode is mixed and thus interpolates between the two other modes. The apparent collapse of all scaled $p^{*}$ data at small strain, with similar pre-factors $p_{0} \approx 0.040$, is interesting since, irrespective of the applied deformation mode - purely isotropic, uniaxial, 


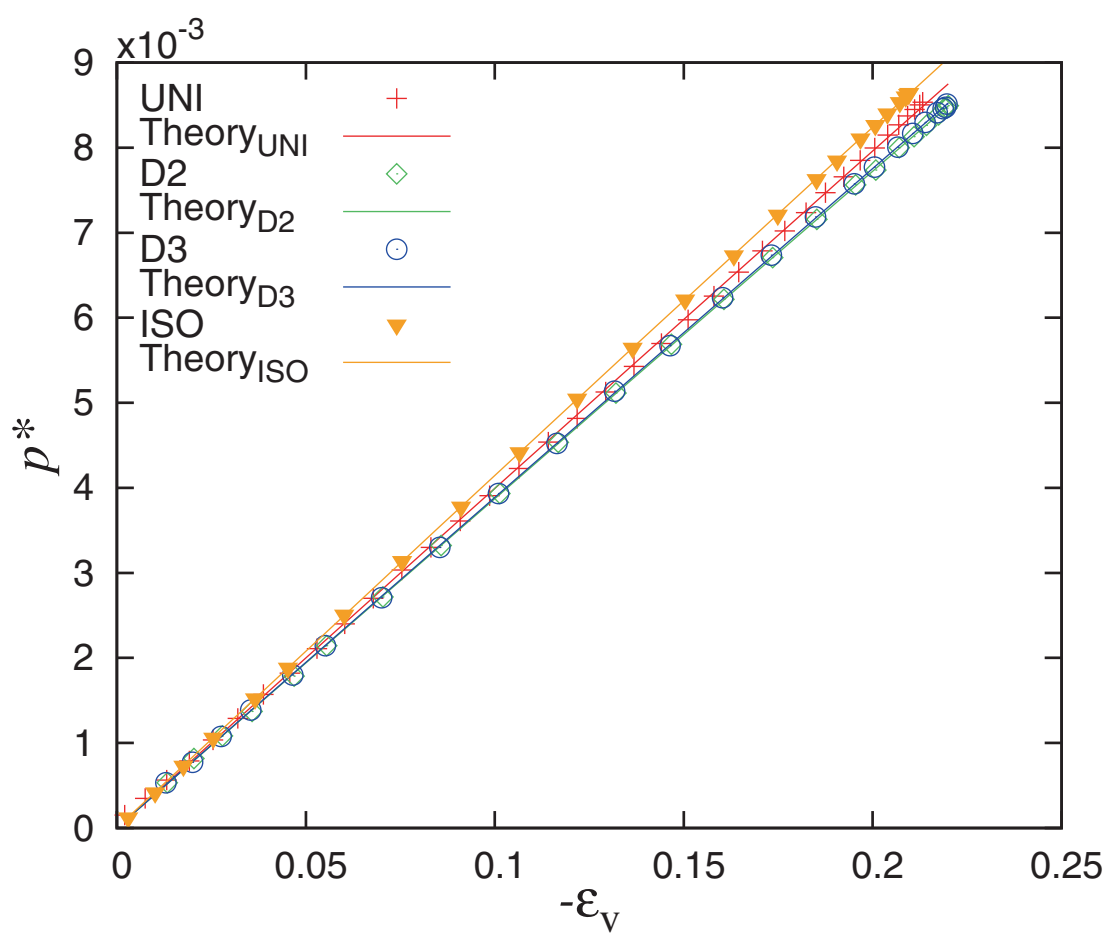

Figure 2.9: The scaled pressure plotted against the (negative) volumetric strain, for the same data as presented in Fig. 2.8. The solid lines are the prediction from Eq. (2.15), using the fits of $p$ and $C$ for each mode.

and D2 or D3 deviatoric, it boils down to a linear relation between $p^{*}$ and $-\varepsilon_{v}$ with a small quadratic correction - not showing small non-linear powers as proposed earlier, e.g. in Ref. [132]. The nonlinearity in $1-v / v_{c}$ is hidden in $v C$, which is actually proportional to the isotropic fabric.

\subsubsection{Isotropic fabric}

The random, isotropic orientation of the contact directions in space was studied in detail in Refs. [69, 179] and is referred to as the contact number density, with $\operatorname{tr}(\mathbf{F})=g_{3} v C$, where $g_{3}$ is of order unity and depends only on the size-distribution (for our case with $w=3$, one has $g_{3} \approx 1.22$ ). Note that $v C$ directly connects to the dimensionless pressure which, remarkably, hides the corrected coordination number and the fraction of rattlers in the relation $C=(1-$ $\left.\phi_{r}\right) C^{*}$, which fully determines $\operatorname{tr}(\mathbf{F})$.

\subsubsection{Evolution of macro-quantities: Deviatoric part}

In the following, we present the evolution of the deviatoric stress ratio (which can be seen as a measure of stress anisotropy) and also the evolution of the structural anisotropy, both as a function of the deviatoric strain. In this sub-section, we will mostly present the raw data 
from deviatoric and uniaxial simulations and their phenomenology, together with the fits of the former (D2 element tests) to calibrate the constitutive model. The constitutive model, as presented in Refs. [128, 131], is presented later in section 2.7, and is used to predict the stress and fabric response under uniaxial (UNI) loading. By doing this, we do not fit the uniaxial tests but qualitatively predict the evolution of stress and fabric.

\subsubsection{Deviatoric Stress}

The deviatoric stress ratio $\left(s_{\mathrm{dev}}=\sigma_{\mathrm{dev}} / P\right.$ ) quantifies the (stress) anisotropy [102]. The loading response of the deviatoric stress ratio for the deformation mode D2, as function of the deviatoric strain, is shown in Fig. 2.10a. Some exemplary results of this dimensionless stress ratio are shown for different volume fractions. The stress grows initially linearly with applied strain until an asymptote (of maximum stress anisotropy) is reached where it remains fairly constant. (The softening behavior after maximal $s_{\mathrm{dev}}$ is ignored in the following, for the sake of brevity, and will be addressed in future studies. The reached asymptote, is referred to as the deviatoric steady state and the initial increase and the approach are well fitted by the exponential relation proposed in Refs. [128, 131] for the biaxial box. Interestingly, the stress response observed from mode D3 (not shown) follows practically the same path as for mode D2, as discussed in section 2.7.

In Fig. 2.10b, uniaxial (compression) simulations are shown, beginning from different initial volume fractions, to a maximum volume fraction defined by similar strain amplitudes. Unlike the deviatoric modes discussed previously, the evolution of the deviatoric stress ratio during uniaxial compression leads to larger fluctuations that do not allow the clear observation of a possible increase or decrease in the saturation regime. This difference is because the uniaxial deformation mode is not a volume conserving mode with continuously increasing pressure in contrast, for example to a similar mode $\mathrm{D} 3$, where $\sigma_{z z}$ is increasing and $\sigma_{x x} \approx \sigma_{y y}$ are decreasing, such that the pressure remains (almost) constant. The corresponding solid lines in the plot represent the predictions of the constitutive relations in Eq. (2.17), with the parameters obtained from the deviatoric modes, D2 and D3, as explained in detail in section 2.7 .

Moreover, as the deviatoric strains increase from the uniaxial simulations for different volume fractions, the deviatoric stress ratios, $s_{\text {dev }}$ also increase (sometimes with a maximum). For smaller volume fractions the values are higher, similar to what we observe in Fig. 2.10a. It is astonishing that uniaxial deformation for different initial volume fractions, lead to convergence and collapse after $7.5 \%$ deviatoric strain. This feature of the uniaxial simulations is also captured by the anisotropy model in section 2.7.3. 


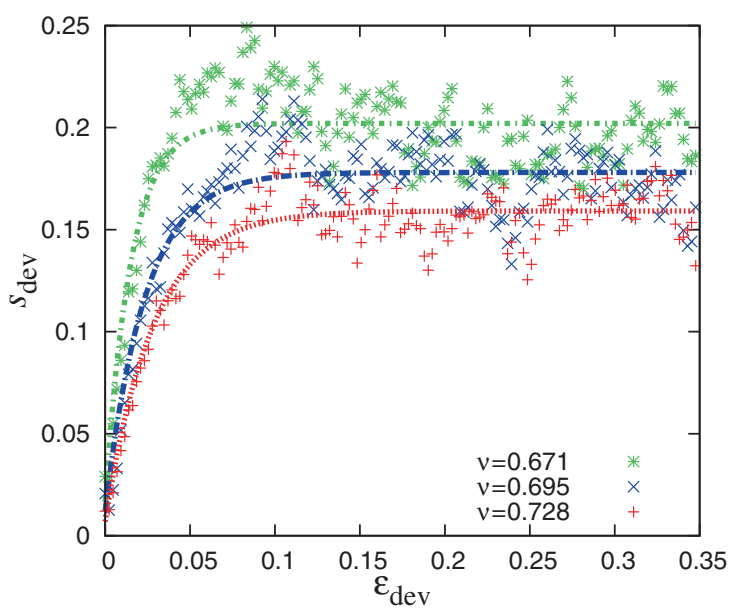

(a)

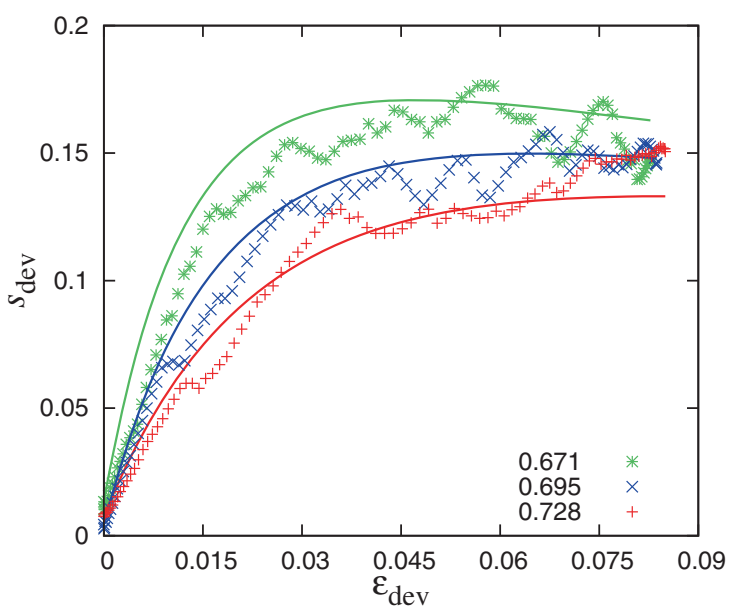

(b)

Figure 2.10: Deviatoric stress ratio $\left(s_{\mathrm{dev}}=\sigma_{\mathrm{dev}} / P\right)$ plotted against deviatoric strain from the (a) D2 deformation mode and (b) uniaxial compression mode data for initial volume fractions $v_{i}$ during unloading, from which the simulations were performed, as given in the inset. The symbols (' $*$ ', ' $\times$ ' and ' + ') are the simulation data while the solid lines through them represent a fit to the data using Eq. (2.17).

\subsubsection{Deviatoric fabric evolution}

The evolution of the deviatoric fabric, $F_{\mathrm{dev}}$ as a function of the deviatoric strain is shown in Fig. 2.11a for mode D2 simulations with three different volume fractions. It builds up from different random, small initial values to different maximum values. The deviatoric fabric builds up faster at lower volume fractions like the $G^{\text {oct }} / P$ ratio, where $G^{\text {oct }}$ is the octahedral shear modulus [15], and the maximal values also are larger for smaller volume fractions. The evolution of the deviatoric fabric for the D3 mode is not shown, since it is practically identical to the D2 mode, implying that the fabric evolution is pretty much insensitive to the deviatoric deformation protocol employed, as was observed before also for the stress ratio.

Fig. 2.11b shows the evolution of the deviatoric fabric for the uniaxial deformation mode from the same simulations as presented in section 2.6.2.1. The deviatoric fabric builds up as the deviatoric strain (and the volume fraction) increases. After a few percent of deviatoric strain, it begins to saturate even though a slight decreasing trend is seen towards the end of the simulation. The convergence of the deviatoric stress after large strain for different volume fractions, as seen in Fig. 2.10b, does not appear so clearly for the deviatoric fabric. The solid lines representing the theoretical prediction of the constitutive relation in Eq. (2.17) agree qualitatively well, but over-predict the deviatoric fabric for larger strains. Their analytical form and parameters involved will be discussed in detail in section 2.7 below. 


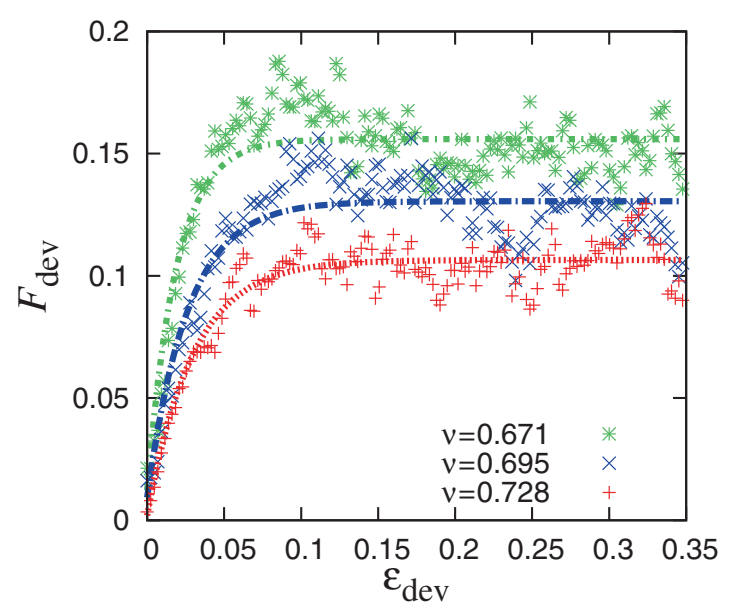

(a)

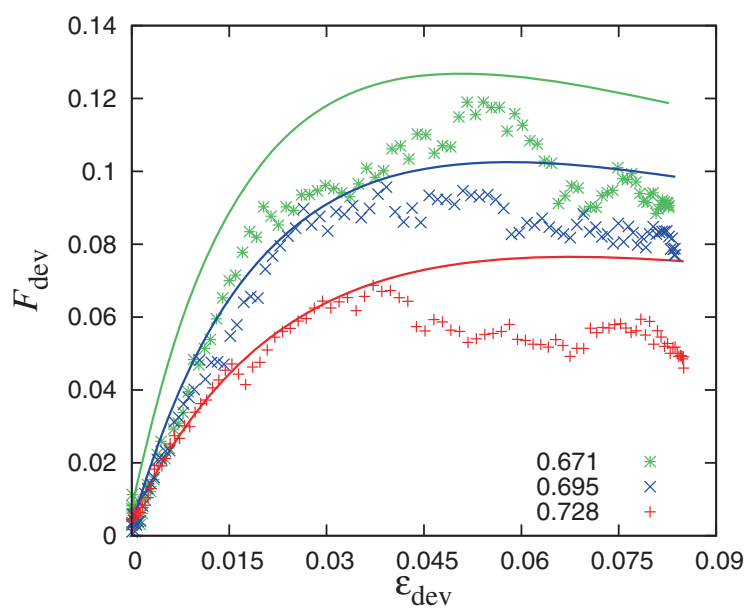

(b)

Figure 2.11: Deviatoric fabric plotted against deviatoric strain from the (a) D2 deformation simulations of Fig. 2.10a and (b) uniaxial deformation simulations in Fig. 2.10b. The symbols (' $*$ ', ' $x$ ' and ' + ') are the simulation data while the solid lines through them represent a fit to the data using Eq. (2.18).

\subsection{Theory: Macroscopic evolution equations}

Constitutive models are manifold and most standard models with wide application fields, like elasticity, elasto-plasticity, or fluid-/gas-models of various kinds, were applied also to granular flows - sometimes with success, but typically only in a very limited range of parameters and flow conditions; for overviews see e.g. Ref. [126, 140]. Only for rapid granular flows, the framework of kinetic theory is an established tool with quantitative predictive value but it is hardly applicable in dense, quasi-static and static situations [125]. Further models, like hyper- or hypo-elasticity, are complemented by hypo-plasticity and the so-called granular solid hydrodynamics, where the latter provide incremental evolution equations for the evolution of stress with strain, and involve limit-states [136], instead of a plastic yield surface as in plasticity theory, where a strict split between elastic and plastic behavior seems invalid in granular materials, see e.g. Ref. [5]. More advanced models involve so-called nonassociated / non co-axial flow rules, where some assumptions on relations between different tensors are released, see e.g. Ref. [196]. While most of these theories can be or have been extended to accommodate anisotropy of the micro-structure, only very few models account for an independent evolution of the microstructure as for example Refs. [128, 190], as found to be important in this study and many others.

In the following, we use the anisotropy constitutive model, as proposed in Refs. [128, 131], 
generalized for a $\mathcal{D}$-dimensional system:

$$
\begin{aligned}
\delta P & =\mathcal{D} B \delta \varepsilon_{\mathrm{v}}+A S \delta \varepsilon_{\mathrm{dev}} \\
\delta \sigma_{\mathrm{dev}} & =A \delta \varepsilon_{\mathrm{v}}+\mathcal{D} G^{\mathrm{oct}} S \delta \varepsilon_{\mathrm{dev}} \\
\delta A & =\beta_{A} \operatorname{sign}\left(\delta \varepsilon_{\mathrm{dev}}\right)\left(A^{\mathrm{max}}-A\right) \delta \varepsilon_{\mathrm{dev}}
\end{aligned}
$$

In its simplest form, the model involves only three elastic moduli: the classical bulk modulus $B$ [69], the octahedral shear modulus $G^{\text {oct }}$, and the new variable "anisotropy modulus" $A$, evolving independently of stress with deviatoric strain. Due to $A$, the model provides a cross coupling between the two types of stress and strain in the model, namely the hydrostatic and the shear (deviatoric) stresses react to both isotropic and deviatoric strains. $S=\left(1-s_{\mathrm{dev}} / s_{\mathrm{dev}}^{\max }\right)$ is an abbreviation for the stress isotropy with $s_{\mathrm{dev}}=\sigma_{\mathrm{dev}} / P$. The parameter $s_{\mathrm{dev}}^{\max }$ resembles the macroscopic friction and $\beta_{s}$ is the growth rate of $s_{\mathrm{dev}}$ with deviatoric strain $\varepsilon_{\mathrm{dev}}$. The parameter $A^{\max }$ in the evolution equation of $A$ represents the maximum anisotropy that can be reached at saturation, and $\beta_{A}$ determines how fast the asymptote is reached (growth rate). Both $A^{\mathrm{max}}$ and $\beta_{A}$ are model parameters for the anisotropy modulus and can be extracted from fits to the macroscopic, average simulation results. Note that the evolution of $A$ is assumed to be kinematic, i.e., not explicitly dependent on pressure, but there is a possible volume fraction dependence of $\beta_{A}$ and $A^{\text {max }}$, as detailed below.

In the following, we test the proposed model by extracting the model parameters from various volume conserving deviatoric simulations as a function of volume fraction $v$. The calibrated model is then used to predict the uniaxial deformation behavior (see the previous section). In short, it is based on the basic postulate that the independent evolution of stress and structure is possible. It comes together with some simplifying assumptions as:

(i) the new macroscopic field $A$ is proportional to the microscopic rank-two deviatoric fabric $F_{\mathrm{dev}}$, so that they have the same non-dimensional growth rates $\beta_{F}=\beta_{A}$;

(ii) both $A$ and $s_{\mathrm{dev}}$ - to lowest order, i.e., neglecting additional (missing) terms in Eqs. (2.16) - approach their limit states exponentially fast;

(iii) only one anisotropy modulus $A$ is sufficient (valid in $2 \mathrm{D}$, questionable in $3 \mathrm{D}$, possibly $A_{1}$ and $A_{2}$ ).

All this leads to Eqs. (2.17) and (2.18) below. (We use these two equations as empirical fit functions, since they are special cases of the complete constitutive model with anisotropy, and use the fit-result to predict one other solution of the (simplified) theory for another deformation mode.)

\subsubsection{Reduced theoretical model}

The reduced model consists of two evolution equations for the deviatoric stress ratio $s_{\mathrm{dev}}$, related to the mobilized macroscopic friction, and the deviatoric fabric $F_{\mathrm{dev}}$, based on DEM 
observations in 2D, see Ref. [119, 120]. For volume conserving pure shear, Fig. 2.11 show that $s_{\mathrm{dev}}$ and $F_{\mathrm{dev}}$ grow non-linearly until they approach exponentially a constant value at steady state, with fluctuations, where the material can be indefinitely sheared without further change. As discussed in Ref. [128], the coupled evolution equations (2.16) are (with above assumptions) consistent with $s_{\mathrm{dev}}$ approximated by:

$$
s_{\mathrm{dev}}=s_{\mathrm{dev}}^{\max }-\left(s_{\mathrm{dev}}^{\max }-s_{\mathrm{dev}}^{0}\right) e^{-\beta_{s} \varepsilon_{\mathrm{dev}}},
$$

where $s_{\mathrm{dev}}^{0}$ and $s_{\mathrm{dev}}^{\max }$ represent the initial and maximum values of $s_{\mathrm{dev}}$ and $\beta_{s}$ is its growth rate. Similarly, the deviatoric fabric is approximated by:

$$
F_{\mathrm{dev}}=F_{\mathrm{dev}}^{\mathrm{max}}-\left(F_{\mathrm{dev}}^{\mathrm{max}}-F_{\mathrm{dev}}^{0}\right) e^{-\beta_{F} \varepsilon_{\mathrm{dev}}}
$$

where $F_{\mathrm{dev}}^{0}$ and $F_{\mathrm{dev}}^{\mathrm{max}}$ represent the initial and maximum (saturation) values of the deviatoric fabric and $\beta_{F}$ is its rate of change. To study the variation of the parameters $s_{\mathrm{dev}}^{\max }, \beta_{s}, F_{\mathrm{dev}}^{0}$ and $F_{\operatorname{dev}}^{\max }$ with volume fraction $v$ during deviatoric deformation, we perform several isochoric simulations at different volume fractions $v_{i}$, and obtain the coefficients as shown in Figs. 2.12 and 2.13 from fits to Eqs. (2.17) and (2.18).

As final step, but not shown in this chapter, in order to relate the macroscopic anisotropy (modulus) $A$ to the evolution of the deviatoric fabric $F_{\mathrm{dev}}$, one can measure the elastic modulus $A$ directly. For this the sample is deformed a little (either isotropically or purely deviatoric) at various different stages along the (large strain) deviatoric paths for D2 and D3 deformation modes. Here, we only note that a linear relation is found such that:

$$
A \approx a_{0}^{*} F_{\mathrm{dev}} \frac{P v_{c}^{2}}{\left(v-v_{c}\right)} \approx \frac{a_{0} k}{2\langle r\rangle} F_{\mathrm{v}} F_{\mathrm{dev}},
$$

where $a_{0}=0.137$ is a combination of numerical constants including $g_{3}, p_{0}$.

\subsubsection{Fitting of deviatoric deformations}

For various deviatoric D2 and D3 simulations with different volume fraction, using Eq. (2.17), we obtain the variation of $s_{\mathrm{dev}}^{\max }$ and $\beta_{s}$ with $v$, Fig. 2.12. The factor $s_{\mathrm{dev}}^{\max }$ decreases with increasing volume fraction $v$ and a similar decreasing trend for $\beta_{s}$ is seen with some larger scatter. Both $s_{\mathrm{dev}}^{\max }$ and $\beta_{s}$ seem to saturate towards a finite limit for large volume fractions and these values can be extrapolated by the fitting procedure, described later in this section. The two parameters $s_{\mathrm{dev}}^{\max }$ and $\beta_{s}$, as obtained from the different D2 and D3 deformation modes are very similar. This is not astonishing: the same net deviatoric strain applied in the two modes leads to the same net deviatoric stress ratio response, even though the shape of deformation is different.

Figure 2.13(a) shows the variation of $F_{\text {dev }}^{\max }$ with volume fraction for the same simulations as in Fig. 2.12, where the two deviatoric deformation modes D2 and D3 almost collapse on 


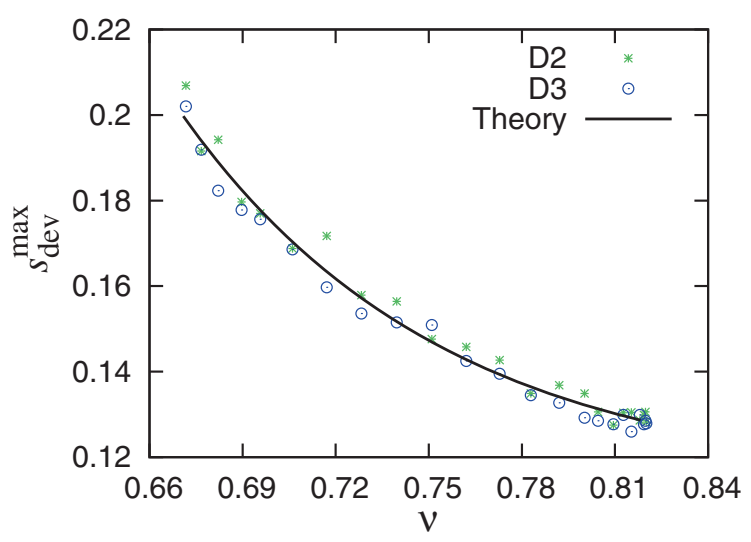

(a)

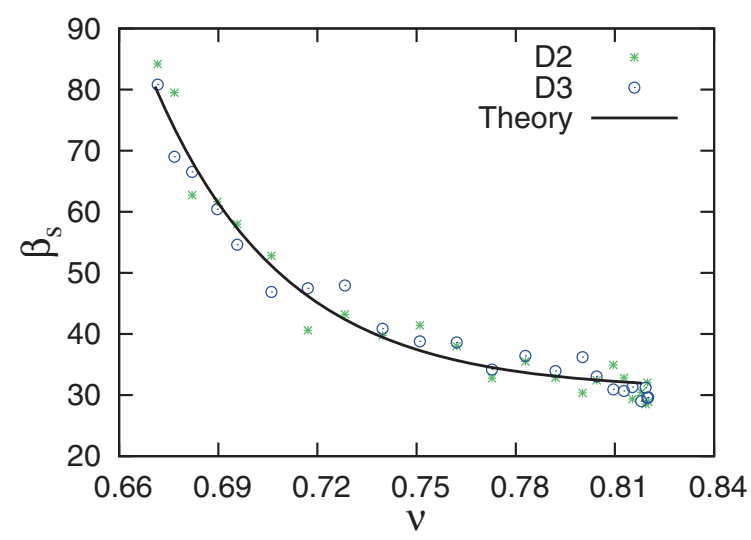

(b)

Figure 2.12: Comparison of evolution parameters from Eq. (2.17): the maximum normalized deviatoric stress $s_{\mathrm{dev}}^{\max }$ and the growth rate $\beta_{s}$ plotted against volume fraction $v$ for the D2 and D3 deviatoric modes. Each point represents a unique simulation; the green ' $*$ ' represent the D2 mode while the blue ' $\odot$ ' represent the D3 mode. The solid black line is the proposed analytical form in Eq. (2.20), with parameters given in Table 2.2.

each other. $F_{\mathrm{dev}}^{\max }$ decreases strongly with volume fraction $v$ for the two modes. For higher volume fractions the motion of spheres is more constrained by more contacts and hence the anisotropy developed in the system is smaller. Figure 2.13(b) shows a similar decreasing behavior of $\beta_{F}$ with volume fraction $v$, where stronger scatter is seen. The analytical fits of the normalized stress parameters $\left(s_{\mathrm{dev}}^{\max }\right.$ and $\beta_{A}$ ) are shown for comparison. A different behavior of the normalized stress and the deviatoric fabric with respect to both parameters (maximum saturation value and the evolution rate), proves that stress and fabric evolve independently with deviatoric strain [110], as is the basic postulate for the anisotropic constitutive model.

For the fit, we propose a generalized analytical relation for both the stress parameters $s_{\mathrm{dev}}^{\max }$, $\beta_{s}$ and the fabric parameters $F_{\mathrm{dev}}^{\max }, \beta_{F}$, obtained from various different volume conserving deviatoric D2/D3 simulations. The dependence of the parameters on volume fraction $v$, is well described by the general relation:

$$
Q=Q_{\max }+Q_{\mathrm{v}} e^{\left(-\alpha\left(\frac{v}{v_{c}}-1\right)\right)},
$$

where $Q_{\max }, Q_{\mathrm{v}}$ and $\alpha$ are the fitting parameters with values presented in Table 2.2, $v$ is the volume fraction and $v_{c} \approx 0.6653$ is chosen as the jamming volume fraction, see Table 2.1. For all four parameters, the $Q_{\max }$ values are the limit for large volume fractions, while $Q_{c}=Q_{\max }+Q_{\mathrm{v}}$ represents the limit at $v \rightarrow v_{c}$, and $\alpha$ is the rate of variation (decay) with the volume fraction increasing above $v_{c}$. We assume, as consistent with the data, that the structural anisotropy parameters $F_{\operatorname{dev}}^{\max }$ and $\beta_{F}$ tend to 0 as the volume fraction increases, therefore keeping $Q_{\max }=0$ in their fitting functions. Eq. (2.20) represents the solid black lines shown in Figs. 2.12 and 2.13, with coefficients given in Table 2.2. 


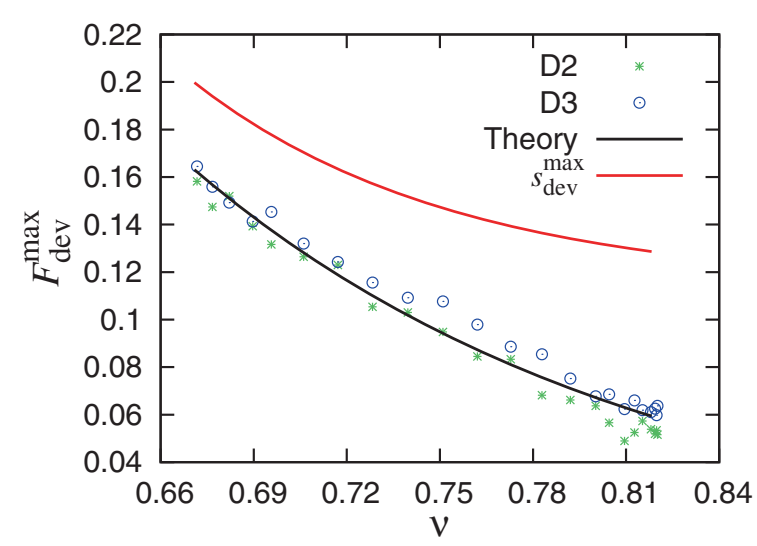

(a)

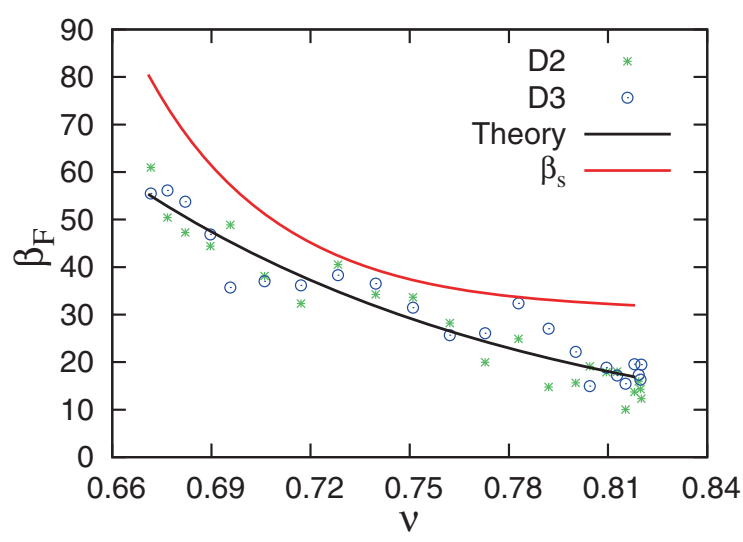

(b)

Figure 2.13: Comparison of evolution parameters from Eq. (2.18): the maximum anisotropy $F_{\text {dev }}^{\max }$ and the growth rate $\beta_{F}$ plotted against volume fraction $v$ for the $\mathrm{D} 2$ and D3 deviatoric modes. The solid black line is the proposed theory, Eq. (2.20), for $F_{\mathrm{dev}}^{\max }$ and $\beta_{F}$ respectively, while the red lines are the corresponding parameters $s_{\operatorname{dev}}^{\max }$ and $\beta_{s}$ from Eq. (2.17), in Fig. 2.12 .

\begin{tabular}{c|c|c|c} 
Evolution parameters & $Q_{a}$ & $Q_{c}$ & $\alpha$ \\
\hline$s_{\mathrm{dev}}^{\max }$ & 0.1137 & 0.09166 & 7.916 \\
\hline$\beta_{s}$ & 30.76 & 57.00 & 16.86 \\
\hline$F_{\mathrm{dev}}^{\max }$ & 0 & 0.1694 & 4.562 \\
\hline$\beta_{F}$ & 0 & 57.89 & 5.366
\end{tabular}

Table 2.2: Fitting coefficients for the parameters in Eqs. (2.17) and (2.18) with $v_{c}=0.6653$

\subsubsection{Prediction of uniaxial deformation}

We used the parameters determined from the deviatoric simulations presented in Table 2.2, to predict the behavior of uniaxial simulations in subsection 2.6.2, where the volume fraction is changing with deviatoric strain and hence dependence on $v$ is needed to properly describe the deformation path.

Fig. 2.10b shows the normalized deviatoric stress $s_{\mathrm{dev}}$ against deviatoric strain $\varepsilon_{\mathrm{dev}}$ for uniaxial deformations starting from three different volume fractions $(v=0.671,0.695$ and 0.728$)$, and compared with the predictions of Eq. (2.17) with coefficients $s_{\mathrm{dev}}^{\max }(v)$ and $\beta_{s}(v)$ taken from Eq. (2.20) and coefficients from Table 2.2. The proposed model, although in its simplified version, is able to properly capture the behavior of the material qualitatively, approaching exponentially a maximum value and then decreasing due to the volume fraction dependence of the parameters. Note that the softening present in some of the deviatoric DEM data, is on purpose not plugged into the model as a constraint, which renders the softening 
present in the uniaxial deformation data as a valuable prediction of the model. Furthermore, the convergence of $s_{\mathrm{dev}}$ for uniaxial loading simulations with different initial volume fraction at large strains, as discussed in section 2.6.2.1, is also well captured by the theoretical model with calibrated parameters from the deviatoric simulations, where this does not happen.

Figure $2.11 \mathrm{~b}$ shows the evolution of deviatoric fabric, $F_{\mathrm{dev}}$, with deviatoric strain, $\varepsilon_{\mathrm{dev}}$, for uniaxial deformations - as above - together with the predictions of Eqs. (2.18) and (2.20). The model is still able to qualitatively describe the behavior of the deviatoric fabric, but with order of $30 \%$ over-prediction. The complete coupled model needs to be used and possibly improved, is presented in Ref. [106].

\subsection{Conclusions and Outlook}

The discrete element method has been used to investigate the bulk response of polydisperse, linear, frictionless sphere packings in 3D to various deformation modes in terms of both their micro- and macroscopic response. Main goal was to present a procedure to calibrate a constitutive model with the DEM data and then use the same to predict another simulation (mode). The (overly) simple material (model) allows to focus on the collective/bulk response of the material to different types of strain, excluding complex effects due to normal or tangential non-linearities. Therefore, the present study has to be seen as a reference "lower-limit" and the procedure rather than the material is the main subject.

We focused on strain-controlled loading and unloading of isotropic, uniaxial and two deviatoric (pure shear) type deformation modes (D2 and D3). Experimentally most difficult to realize is isotropic deformation, while both uniaxial and deviatoric modes can be realized in various element tests where, however, often mixed strain- and stress-control is applied. Both micro-mechanical and coarse grained macroscopic properties of the assemblies are discussed and related to each other. The study covers a very wide range of isotropic, uniaxial and deviatoric deformation amplitudes and thus practically all volume fractions with mechanically stable packings - except for those very close to the jamming volume fraction and higher than about $10 \%$ contact deformation, above which DEM pair contact models are questionable.

\subsubsection{Microscopic quantities}

The microscopic coordination number $C$, defined as the ratio of the total number of contacts to the total number of particles, has been analyzed as function of volume fraction and deviatoric strain. By disregarding particles with less than four contacts (called rattlers), the corrected coordination number $C^{*}$ is well described by Eq. (2.11) for all deformation modes (since the particles are frictionless). For the uniform size-distribution used here, the fraction of rattlers shows an exponentially decaying trend towards higher volume fractions, very similar for all modes, see Eq. (2.12), and Table 2.1. These analytical relations provide $a$ 
prediction for the coordination number $C=\left(1-\phi_{r}\right) C^{*}$ that, notably, shows up in the macroscopic relations for both pressure and isotropic fabric, in combination with $v$, instead of $C^{*}$. (Note that $C^{*}$ is better accessible to theory, while $v C$ is related to the wave-propagation speed, which is experimentally accessible, while both are linked by the fraction of rattlers, which was already identified as a control parameter of utmost importance [21].)

A small but systematic difference in the $C^{*}$ and $\phi_{r}$ parameters appears for the different deformation modes. Most important, the jamming volume fraction $v_{c}$ is not a single, particular volume fraction, but we observe a range of jamming volume fractions dependent on the deformation modes, i.e., the "history" of the sample. Over-compression leads to an increase of $v_{c}$, i.e., to a better, more efficient packing. Subsequent deviatoric (pure shear) deformations slightly reduce the jamming volume fraction of such a previously over-compressed packing, causing it to become less efficiently packed. Note that more/less efficient packing is reflected by a large/small jamming volume fraction and, inversely, small/large coordination numbers. The effects described are more pronounced as the volume fraction becomes lower. For example, a slight increase in the fraction of rattlers due to deviatoric deformations is also reported, as consistent with the decrease in coordination number. Deviatoric modes (shear) can create more rattlers, while isotropic modes (compression) reduce the fraction of rattlers.

\subsubsection{Macroscopic quantities}

When focusing on macroscopic quantities, an important result from this study is that at small strains, the uniaxial, deviatoric and isotropic modes can be described by the same analytical pressure evolution Eq. (2.15), with parameters given in Table 2.1, evidenced by the collapse of the data from these deformation modes on each other when the scaled pressure is plotted as a linear function of the volumetric strain. This linearity is due to the scaling with the non-linear terms, $p^{*} \propto p /(v C)$ in particular. Thanks to the linear contact model used allows to conclude that the non-linear (quadratic) corrections are due to the structural rearrangements and non-affine deformations. A deviation of the scaled deviatoric and uniaxial from isotropic pressure data appears at larger strains, due to the build up of anisotropy in the system caused by deviatoric strain, obviously not present in the isotropic deformation mode. The good match of the data suggests an advantage of the "cheaper" uniaxial (and deviatoric) deformation modes over the experimentally difficult to realize isotropic deformation mode. (Three walls have to be moved simultaneously in the isotropic case, while a less complicated set-up is required for the other modes.)

The evolution of the deviatoric stress ratio (the deviatoric stress scaled with the isotropic pressure) as a function of the deviatoric strain is developing almost independently of the volume conserving deformation modes, when the deviatoric magnitude is defined in a similar fashion to the second deviatoric invariant [196], for all quantities studied. The deviatoric stress builds up with increasing deviatoric strain until a steady state is reached (where we 
do not focus on peak- and softening-behavior in this study, which is becoming more pronounced closer to the jamming volume fraction). Starting from isotropic initial configurations, we also show that the slope $\left(G^{\text {oct }} / P\right)$ of the normalized deviatoric stress as function of deviatoric strain decreases with increasing volume fraction, unlike the shear modulus $G^{\text {oct }}$, which increases with volume fraction. This indicates, that the pressure (and bulk modulus $B)$ are dependent on volume fraction "stronger" than the shear modulus.

From the macroscopic data, one observes that deviatoric and isotropic stresses and strains are cross-coupled by the structural anisotropy. The latter is quantified by the deviatoric fabric, which is proportional to the bulk-anisotropy modulus/moduli $A$, as relevant for the constitutive model. Cross-coupling means that - in the presence of structural anisotropy isotropic strain can cause deviatoric stress responses and deviatoric strain can cause isotropic stress responses (dilatancy or compactancy). The structural anisotropy behaves very similar to the deviatoric stress ratio with deviatoric strain, but has different response rates as the latter. The response rates of the anisotropy of both stress and structure with deviatoric strain are functions of volume fraction and, most important, different from each other.

\subsubsection{Constitutive model calibration}

As first step, the parameters of the simplest constitutive model that involves anisotropy, as proposed in Refs. [128, 131] for 2D, have been extracted from DEM data for calibration. From the isotropic deformation mode, one can extract the bulk-modulus $B$, as done in Ref. [69]. From the volume conserving D2 and D3 modes, by fitting the idealized evolution equations for shear stress in Eq. (2.17), the macroscopic friction, $s_{\mathrm{dev}}^{\max }$, and the deviatoric stress rate $\beta_{s}$ can be inferred as functions of the volume fraction, entering the shear modulus $G^{\text {oct }}$. Similarly, the fit of Eq. (2.18) provides a relation for the maximum structural anisotropy at steady state, $F_{\mathrm{dev}}^{\max }$, and the fabric rate $\beta_{F}$, as functions of volume fraction. A relation between the deviatoric fabric and the anisotropy modulus/moduli $A$, in the model is finally needed to close the system and allow to integrate the coupled evolution equations for stress and structure.

As second step, and major result, the constitutive model calibrated on deviatoric data was used to predict qualitatively (and to some extent also quantitatively) both the stress and fabric evolution under uniaxial deformation. This is very promising, since the basic qualitative features are caught by the model, even though it was used in a very idealized and short form, with only main new ingredient the single anisotropy modulus $A \propto F_{\mathrm{dev}}$. Several additional terms of assumed lesser magnitude are ignored and have to be added to complete the model, see Refs. [131, 190, 196] and references therein, in further research. (For example, an objective tensorial description of stress, strain and fabric, involves also the third tensor invariants. Alternatively/equivalently, these deviatoric tensors can be completely classified by their shape factors in their respective eigen-systems, which allows to distinguish all possible 
deformation and response modes in 3D.)

In this chapter, we have reviewed and presented new results for frictionless particles undergoing isotropic, uniaxial, and (pure) shear deformation. Since the particles are too idealized, they provide insufficient freedom for direct application to practical systems, where shape, friction, and other non-linearities are relevant. However, they form the basic reference study with details given on the calibration procedure that yields a constitutive model with satisfactory predictive quality. Therefore, the next steps in our research will involve more realistic contact models with friction, cohesion, and other physically meaningful material parameters. Only then, the validity of the analytical expressions, which predict well the phenomenology, and the observations for pressure as well as the scaling arguments presented for the deviatoric stress and fabric, can be tested for realistic frictional, cohesive systems.

\subsubsection{Outlook}

Laboratory element test experiments should also be performed with the biaxial box to validate the simulation results with realistic material properties. Macroscopic quantities that can be readily obtained experimentally - for example the pressure-volume fraction relation and the shear stress evolution with deviatoric (shear) strain - can then be compared with simulation data. Moreover, the work underlines the predictive power of constitutive models with anisotropy, see Refs. [128, 131, 190], that can be further tested, validated and extended with more advanced physical and numerical experiments.

Given the detailed insights from DEM, the (missing) terms and the parameters for the constitutive models can now be further analyzed to perform the rigorous micro-macro transition. Open questions are as follows:

(i) the validity of the $2 \mathrm{D}$ model in $3 \mathrm{D}$, related to missing terms and parameters,

(ii) the validity of global versus local coarse-graining, i.e., the scale of the micro-macro transition [101],

(iii) the microscopic (restructuring) and macroscopic (non-affine motions) origins of the peak- and softening phenomenology at the lower volume fractions, related to the (inhomogeneity of the packings,

(iv) the validity of the model predictions for strain-reversal and cyclic deformations, and

(v) the possible dependence of the moduli in the constitutive relations on other quantities (e.g., pressure) than the volume fraction, as focused on in this study.

For future application, the present calibration procedure should be checked also for other materials and applied to other element tests, among which there are (cylindrical) triaxial tests, ring-shear tests and also avalanche flow experiments like in a rotating drum, all of which are more widely available than the "academic" biaxial box. At the end the material properties and parameters should not depend on the element test chosen and the predictive value of the model(s) should be proven for more than only one validation test, be it another 
element test or a real-size or lab-scale process like, e.g., granular flow in a silo or during a landslide.

\section{A Shape-factors $\zeta$, for the different deformation modes}

\begin{tabular}{c||l|l|l}
\hline \hline Mode & $\begin{array}{l}\text { Strain-rate tensor } \\
\text { (main diagonal) }\end{array}$ & $\begin{array}{l}\text { Deviatoric strain- } \\
\text { rate (magnitude) }\end{array}$ & $\begin{array}{l}\text { Shape factor } \\
\zeta=\left(\epsilon_{\mathrm{d}}^{(2)} / \epsilon_{\mathrm{d}}^{(1)}\right)\end{array}$ \\
\hline ISO & $\dot{\varepsilon}_{\mathrm{v}}(-1,-1,-1)$ & $\dot{\varepsilon}_{\mathrm{dev}}=0$ & n.a. \\
UNI & $\dot{\epsilon}_{\mathrm{u}}(0,0,-1)$ & $\dot{\varepsilon}_{\mathrm{dev}}=\dot{\varepsilon}_{\mathrm{u}}=\dot{\epsilon}_{z z}$ & 1 \\
$\mathrm{D} 2$ & $\dot{\epsilon}_{\mathrm{D} 2}(1,0,-1)$ & $\dot{\varepsilon}_{\mathrm{dev}}=\sqrt{3} \dot{\epsilon}_{\mathrm{D} 2}$ & 0 \\
$\mathrm{D} 3$ & $\dot{\epsilon}_{\mathrm{D} 3}(1 / 2,1 / 2,-1)$ & $\dot{\varepsilon}_{\mathrm{dev}}=(3 / 2) \dot{\epsilon}_{\mathrm{D} 3}$ & 1 \\
\hline
\end{tabular}

Table 2.3: Summary of the deformation modes, and the deviatoric strain-rates $\dot{\varepsilon}_{\mathrm{dev}}$, as well as shape-factors, $\zeta$, for the different modes, in the respective tensor eigensystem, with eigenvalues $\epsilon_{\mathrm{d}}{ }^{(1)}$ and $\epsilon_{\mathrm{d}}{ }^{(2)}$ as defined in section 2.4.2.1. 


\title{
CHAPTER 3
}

\section{Effects of polydispersity on the behavior of granular assemblies*}

\begin{abstract}
The micromechanical and macromechanical behavior of idealized granular assemblies, made by linearly elastic, frictionless, polydisperse spheres, are studied in a periodic, triaxial box geometry, using the discrete element method. Emphasis is put on the effect of polydispersity under purely isotropic loading and unloading, deviatoric (volume conserving) pure shear, and uniaxial compression paths. We show that the isotropic quantities, namely scaled pressure, coordination number and fraction of rattlers, behave in a very similar fashion as functions of volume fraction, irrespective of the deformation mode applied, but show a systematic dependence on the deformation mode and polydispersity via the respective jamming volume fraction. We present a relation for the jamming volume fraction (and other parameters) as function of the polydispersity and the deformation mode. This confirms that the concept of a single jamming point has to be rephrased to a "wide range" of values, dependent on the microstructure and history of the sample. All this has not only effects for isotropic quantitities but also for the structural anisotropy and its interplay with the deviatoric stress. Therefore, a simplified constitutive model involving structural anisotropy and its evolution with strain is presented and calibrated using model parameters from the volume conserving deviatoric mode. Some of the basic parameters are found to
\end{abstract}

*. Based on N. Kumar, O. I. Imole, V. Magnanimo, and S. Luding. Effect of polydispersity on the micro-macro behavior of granular assemblies under different deformation paths. Particuology, (http://dx.doi.org/10.1016/j.partic.2013.07.011), 2013 
depend on the polydispersity of the sample through the jamming volume fraction, others do not. The predictive power of the calibrated model is confirmed by comparison with an independent test, namely the uniaxial compression. The important features of the uniaxial experiment are captured and a qualitative prediction for the evolution of stress and fabric is confirmed involving a "softening" regime in both stress and fabric - stronger for the latter - that was not prescribed into the model a-priori.

\subsection{Introduction and Background}

Granular materials are widely used as raw materials in various industries, including pharmaceutical, mining, chemical, agricultural, household products and food industries. In many of these industries, processes involving milling, segregation, fragmentation, agglomeration, filtration and sieving, among others are common and often lead to the generation of granular systems with large size ratios. The optimization of these systems are exceptionally challenging and often requires heuristic assumptions to be made. It is known, however that polydispersity influences the micro-mechanical behavior of granular systems. For example, the shear strength and packing fraction, which are important quantities in determining the stress state and response of granular assemblies have been shown to be influenced by the size ratio of the packing $[34,70,179]$.

On the other hand, the bulk macroscopic behavior of granular systems originates from the contact force network between their constituent particles. The contact force networks, even for systems with a uniform size distribution, are mostly inhomogeneous leading to many interesting phenomena [179]. In most recent studies involving the effects of polydispersity, emphasis has been placed on systems with narrow size distributions - ostensibly to limit the effects of long-range structural order - with the exception of a few cases where wider distributions have been reported [49, 147, 202, 203]. Additionally, a micromechanical description, which takes into account the discrete nature of granular systems, is necessary and must be linked to the continuum description, which involves the formulation of constitutive relations - for macroscopic fields. In recent years, several constitutive relations have been proposed in literature [66, 68, 96, 136, 190, 197], but only few take into account the anisotropy that develops when granular systems are subjected to shear deformation $[128,131,159]$ and no study, to our knowledge connects anisotropy and polydispersity.

When a granular assembly is subjected to shear deformation, a buildup of shear stress is observed [41, 130], along with an evolution of the structural anisotropy, which describes the creation and destruction of contacts $[4,5,9,76,104,159,161,175,205]$. In this sense, anisotropy represents a history-parameter for the granular assembly. For anisotropic samples, scalar quantities are not sufficient to fully represent the internal direction dependent contact structure; therefore an extra tensorial quantity has to be introduced, namely the fabric tensor [146]. To gain more insight into the microstructure of granular materials, nu- 
merical studies and simulations on various deformation experiments can be performed, see $[75,159,195-197]$

In this study, we perform parametric studies with the goal of understanding the effects of polydispersity on both microscopic and macroscopic responses of granular assemblies under isotropic, uniaxial and deviatoric deformation conditions. As scalar and tensorial microscopic quantities, we investigate the effects of polydispersity on coordination number, fraction of rattlers and the volumetric and deviatoric fabric. The volumetric part of fabric is the measure of the strength of contact network, while the deviatoric part is the measure of directionality of the contact network. On the macroscopic side, we consider the effects of polydispersity on the scaled pressure and the deviatoric stress. Another goal is to calibrate a constitutive model using parameters from deviatoric volume conserving pure shear simulations ${ }^{1}$ and test the predictive power of the calibrated model on an independent test, namely uniaxial compression test.

We propose an objective definition for deviatoric stress and deviatoric fabric for all possible deformation modes in a triaxial box and present findings on their behavior as a function of deviatoric strain. The parameters obtained from pure isotropic and deviatoric deformations are inserted into a constitutive model to predict uniaxial deformation.

This chapter is organized as follows: The simulation method and parameters used and the generalized averaging definitions for scalar and tensorial quantities are given in section 3.2. The preparation and test procedures are explained in section 3.3. Polydispersity is introduced in subsection 3.4.1 and its effect on the evolution of microscopic quantities, i.e. the non-scaled pressure, coordination number and the fraction of rattlers for the different deformation modes are discussed in subsection 3.4.2. In subsection 3.4.3, the macroscopic quantities (deviatoric stress and deviatoric fabric) and their evolution are studied as functions of polydispersity, volume fraction and deviatoric (shear) strain for the different deformation modes. These results are used to obtain/calibrate the macroscopic model parameters. Section 3.5 is devoted to theory, where we relate the evolution of the microstructural anisotropy to that of stress and strain, as proposed in $[128,131]$, to display the predictive quality of the calibrated model.

\subsection{Numerical simulation}

The Discrete Element Method (DEM) [42] has been used extensively in performing simulations in biaxial and triaxial geometries [50, 99, 121, 190] involving advanced contact models for fine powders [122], or general deformation modes, see [5, 195, 197] and refer-

1. In continuum mechanics, pure shear involves element distortion, whereas in this work, we use it to mean constant volume deviatoric loading, where the principal strain axes have the same orientation as they had in the undeformed state [177]. In this case there is no rotation of the strain principal axes and no distortion/rotation of the same due to deformation. 


\begin{tabular}{llll}
\hline \hline Parameter & Symbol & Value & S.I. Units \\
\hline \hline Time Unit & $t_{u}$ & 1 & $1 \mu \mathrm{s}$ \\
Length Unit & $l_{u}$ & 1 & $1 \mathrm{~mm}$ \\
Mass Unit & $m_{u}$ & 1 & $1 \mu \mathrm{g}$ \\
Number of Particles & $N$ & 9261 & {$[-]$} \\
Average radius & $\langle r\rangle$ & 1 & $1 \mathrm{~mm}$ \\
Polydispersity & $w=r_{\max } / r_{\min }$ & varied $[1-10]$ & {$[-]$} \\
Particle density & $\rho$ & 2000 & $2000\left[\mathrm{~kg} / \mathrm{m}^{3}\right]$ \\
Normal stiffness & $k$ & $10^{5}$ & $10^{8}\left[\mathrm{~kg} / \mathrm{s}^{2}\right]$ \\
Normal Viscosity & $\gamma$ & 1000 & $1[\mathrm{~kg} / \mathrm{s}]$ \\
Background viscosity & $\gamma_{b}$ & 100 & $0.1[\mathrm{~kg} / \mathrm{s}]$ \\
\hline
\end{tabular}

Table 3.1: Summary and numerical values of particle parameters used in the DEM simulations.

ences therein. In this work, however, we restrict ourselves to the simplest deformation tests - namely isotropic, uniaxial and deviatoric modes - and to the linear contact model without friction. A detailed description about the contact model used in the DEM simulations can be found in section 2.2 of Chapter 2. A short summary of the values of the parameters used in DEM simulations is shown in Table 3.1 .

\subsubsection{Microscopic Variables}

The simple definition of coordination number is $C=M / N$, where $M$ is the total number of contacts and $N=9261$ is the total number of particles in the system. If the overlap at a contact between two particles is greater than or equal to zero, i.e., for $\delta \geq 0$, the contact contributes to the force network. The corrected coordination number is $C^{*}=M_{4} / N_{4}$, where, $M_{4}$ is the total number of contacts of the $N_{4}$ particles with at least 4 contacts, and the rattler fraction is $\phi_{r}=\left(N-N_{4}\right) / N[69,84,103]$.

The total volume of particles is $\sum_{\mathcal{P}=1}^{N} V_{\mathcal{P}}=4 \pi N\left\langle r^{3}\right\rangle / 3$, where $\left\langle r^{3}\right\rangle / 3$ is the third moment of the size distribution discussed in detail in subsection 3.4.1 and volume fraction is defined as $v=(1 / V) \sum_{\mathcal{P}=1}^{N} V_{\mathcal{P}}$, where $V$ is the volume of the box. Note that for the calculation of the total volume of particles, the volume which should be subtracted from particle overlaps is neglected $[69,84,103]$. 


\subsubsection{Macroscopic variables}

Here, we focus on defining averaged macroscopic tensorial quantities - including strain, stress- and fabric (structure) tensors - that reveal interesting bulk features and provide information about the state of the packing due to its deformation.

For any deformation, the isotropic part of the infinitesimal strain tensor $\varepsilon_{\mathrm{v}}[69,84]$ is defined as:

$$
\varepsilon_{\mathrm{v}}=\dot{\varepsilon}_{\mathrm{v}} \mathrm{dt}=\frac{\epsilon_{x x}+\epsilon_{y y}+\epsilon_{z z}}{3}=\frac{1}{3} \operatorname{tr}(\mathbf{E})=\frac{1}{3} \operatorname{tr}(\dot{\mathbf{E}}) \mathrm{dt},
$$

where $\varepsilon_{\alpha \alpha}=\dot{\varepsilon}_{\alpha \alpha} \mathrm{dt}$ with $\alpha \alpha=x x, y y$ and $z z$ as the diagonal elements of the strain tensor $\mathbf{E}$ in the Cartesian $x, y, z$ reference system. The trace integral of $3 \varepsilon_{\mathrm{v}}$, denoted as the volumetric strain $\varepsilon_{v}$, i.e. the true or logarithmic strain, i.e., the volume change of the system, relative to the initial reference volume, $V_{0}$.

From the DEM simulations, one can determine the stress tensor as

$$
\boldsymbol{\sigma}=(1 / V) \sum_{c \in V} \mathbf{l}^{c} \otimes \mathbf{f}^{c}
$$

which is an average over the contacts in the volume $V$ of the dyadic products between the branch vector $\mathbf{l}^{c}$ and the contact force $\mathbf{f}^{c}$, where the contribution of the kinetic energy has been neglected $[84,120]$. The isotropic component of the stress is the pressure $P=\operatorname{tr}(\boldsymbol{\sigma}) / 3$.

Besides the stress, we will focus on the fabric tensor in order to characterize the geometry/structure of the static aggregate, defined as

$$
\mathbf{F}=(1 / V) \sum_{\mathcal{P} \in V} V^{\mathcal{P}} \sum_{c \in \mathcal{P}} \mathbf{n}^{c} \otimes \mathbf{n}^{c}
$$

where $V^{\mathcal{P}}$ is the particle volume for particle $\mathcal{P}$, which lies inside the averaging volume $V$, and $\mathbf{n}^{c}$ is the normal unit branch-vector pointing from center of particle $\mathcal{P}$ to contact $c$ [104, 120]. The average isotropic fabric is $F_{\mathrm{v}}=\operatorname{tr}(\mathbf{F})=g_{3} v C$, where $v$ and $C$ are, respectively, the volume fraction, the coordination number, and $g_{3}$ is a function of moments of the size distribution [69, 179], as explained in detail in subsection 3.4.1. We want to highlight that a different formulation for the fabric tensor considers simply the orientation of contacts as follows [146, 173]:

$$
\mathbf{F}^{s} \approx \frac{1}{N_{c}} \sum_{c \in N_{c}} \mathbf{n}^{c} \otimes \mathbf{n}^{c}
$$

where the equality holds for the monodisperse case. where $N_{c}$ is the total number of contacts. The relationship between Eq. (3.3) and Eq. (3.4) is:

$$
\mathbf{F}^{s}=\frac{\mathbf{F}}{g_{3} v C}=\frac{3 \mathbf{F}}{F_{\mathrm{v}}}
$$


In addition to the isotropic components, we use the following definition to quantify the magnitude of the deviatoric parts [104] of tensors $\mathbf{Q}$ (stress $\boldsymbol{\sigma}$, strain $\mathbf{E}$ or fabric $\mathbf{F}$ ) :

$$
Q_{\mathrm{dev}}=\operatorname{Fsgn}(\mathbf{Q}) \sqrt{\frac{\left(Q_{x x}-Q_{y y}\right)^{2}+\left(Q_{y y}-Q_{z z}\right)^{2}+\left(Q_{z z}-Q_{x x}\right)^{2}+6\left(Q_{x y}^{2}+Q_{y z}^{2}+Q_{z x}^{2}\right)}{2}},
$$

where $Q_{x x}, Q_{y y}$ and $Q_{z z}$ are the diagonal components, and $Q_{x y}, Q_{y z}$ and $Q_{z x}$ are the offdiagonal components of any symmetric tensor $\mathbf{Q}$. Fsgn $(\mathbf{Q})$ is the sign function with possible values as $+1,0$ and -1 , whose definition depends on the deformation path (see section 3.4.3). In the case of stress, Eq. (3.6) represents the ideal von Mises stress, $\sigma_{\mathrm{dev}}=\sqrt{3 J_{2}}$, related to the second deviatoric stress invariant $J_{2}$.

When a biaxial or triaxial compression is performed, such that the strain, stress and fabric stay almost coaxial with principal axes parallel to the initial reference system for these modes, the off-diagonal terms become negligible and the diagonal terms coincide with the eigen-values.

\subsection{Preparation and test procedure}

After the (common) initial isotropic preparation, the packing is deformed following three different procedures, namely isotropic, uniaxial and deviatoric element test modes, a detailed procedure can be found in section 2.3 of Chapter 2. For convenience, the definitions of the different modes will be based on their respective strain-rate tensors. Also note that the deformations applied to systems are always 'slow' enough to maintain the quasi-static regime and hence minimize the dynamical effects [75, 84].

\subsection{Polydispersity}

Most granular materials are highly polydisperse in nature. It is known that size polydispersity affects the mechanical behavior of granular systems (e.g., shear strength) as well as their space-filling properties (e.g., packing fraction) [70, 147], which are crucial in many engineering chemical processes like absorption, filtering, etc., see $[6,17,18]$ and references therein. Here we use samples with different degrees of polydispersity to study the effect of increasing polydispersity on the evolution of microscopic and macroscopic parameters during various deformation modes.

\subsubsection{Polydispersity}

We define polydispersity in terms of the width $w=r_{\max } / r_{\min }-$ where $r_{\max }$ and $r_{\min }$ represent the radius of the largest and smallest particles in the overall ensemble of a distribution 
uniform in size $[69,70]$ :

$$
f(r)=\frac{w+1}{2(w-1)\langle r\rangle} \Theta\left(\frac{2 w\langle r\rangle}{w+1}-r\right) \Theta\left(r-\frac{2\langle r\rangle}{w+1}\right),
$$

with the step function $\Theta(x \geq 0)=1$ and $\Theta(x<0)=0$. From the distribution of radius, one can calculate the parameter $g_{3}$ that describes the polydispersity of a 3D spherical system, and is defined [69] up to the $5^{\text {th }}$ moment as:

$$
g_{3} \approx \frac{1-B_{2}+C_{2}+\left(B_{2}-2 C_{2}\right) \frac{\left\langle r^{4}\right\rangle}{\langle r\rangle\left\langle r^{3}\right\rangle}+C_{2} \frac{\left\langle r^{5}\right\rangle}{\langle r\rangle^{2}\left\langle r^{3}\right\rangle}}{1+C_{2}\left[\frac{\left\langle r^{2}\right\rangle}{\langle r\rangle^{2}}-1\right]},
$$

where the constants $B_{2}=1.077$ and $C_{2}=0.2629$ are defined in [69] and $\left\langle r^{n}\right\rangle$ is the $n^{\text {th }}$ moment. Only for the monodisperse situation, one has the simplification $g_{3}=1$ from where it increases with polydispersity $w$ and saturates at high values about 1.627. Fig. 3.1 shows the cumulative distribution function (CDF) for the particle size (radius) $r$. Since the sample used have uniform size distribution, we notice a linear trend in CDF for radius range $r_{\min } \leq$ $r \leq r_{\max }$, and zero for rest.

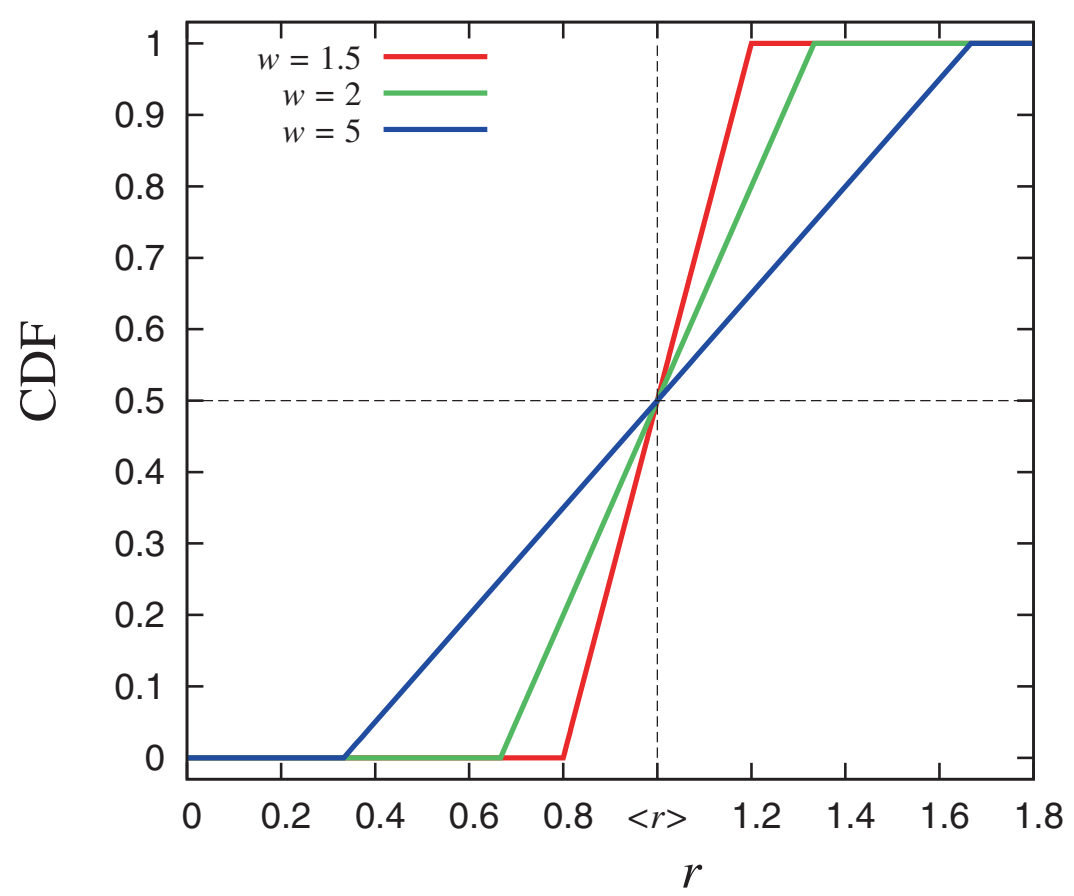

Figure 3.1: Cumulative distribution function (CDF) for radius $r$ for three systems with polydispersity $w=1.5, w=2$ and $w=5$ as shown in the legend, for the same samples as in Fig. 3.2. The horizontal and vertical dashed lines represent the CDF value of 0.5 and mean radius $\langle r\rangle=1$.

In order to study the effect of polydispersity on micro-macro behavior of granular assembly, we prepare ten different packings with polydispersity ranging from $w=1-10$. These pack- 


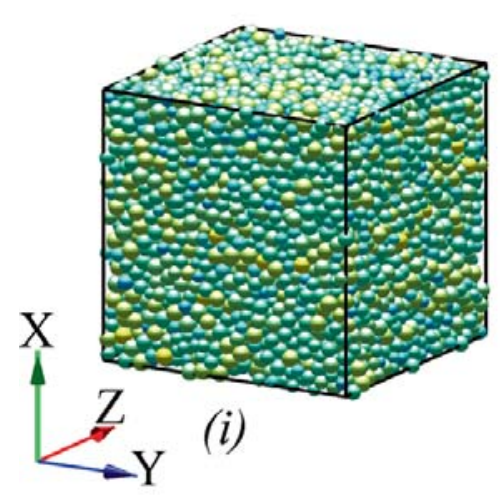

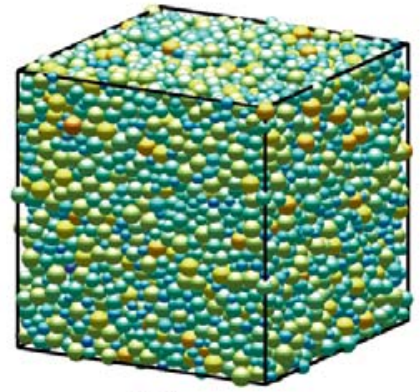

(ii)

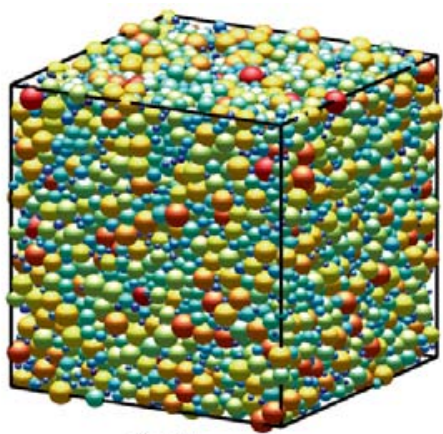

(iii)

Figure 3.2: Snapshots of three systems with polydispersity $(i) 1.5$, (ii) 2 and (iii) 5 respectively with the same volume fraction $v=0.82$. The color code indicates the contacts of the particles: (red: big contacts, blue: no contacts).

ings are deformed following the paths described in section 3.3). As an example, we show in Fig. 3.2 isotropic samples with $w=1.5,2$ and 5 for constant volume fraction $v=0.82$. Note that for the same volume fraction $v$, the volume of the box is higher for higher polydispersity, since $\left\langle r^{3}\right\rangle$ increases with $w$ for fixed $\langle r\rangle=1$. For higher polydispersity, particles of smaller size fill more efficiently in the pore space between larger particles. However, lower polydispersity in packings of granular materials is associated with alterations in the structural order $[147,202] .^{3}$

\subsubsection{Effect of polydispersity on isotropic quantities}

In the following, we will study the influence of polydispersity on scaled pressure, coordination number and fraction of rattlers, during the three deformation paths described above.

\subsubsection{Confining pressure}

Starting from Eq. (3.2), we define the non-dimensional pressure [69, 84] as

$$
p=\frac{2\langle r\rangle}{3 k} \operatorname{tr}(\sigma)
$$

with $\langle r\rangle$ the first radius moment (average radius) and $k$ the spring stiffness defined in section 3.2 , and the scaled pressure is:

$$
p^{*}=\frac{p v_{c}}{v C}=p_{0}\left(-\varepsilon_{\mathrm{v}}\right)\left[1-\gamma_{p}\left(-\varepsilon_{\mathrm{v}}\right)\right]
$$

where $p_{0}, \gamma_{p}$, and the critical volume fraction $v_{c}$ are fit parameters. The scaled pressure $p^{*}$ is independent of the polydispersity and is well represented by the linear relation $p^{*} \approx$

3. Note that results for a uniform radius distribution in polydispersity is presented. The results will be different if the distribution is different e.g., uniform surface or volume distribution. 
$p_{0}\left(-\varepsilon_{\mathrm{v}}\right)$ for small deformations. $p^{*}$ is proportional to the average deformation (overlap) of the particles at a given volume fraction.

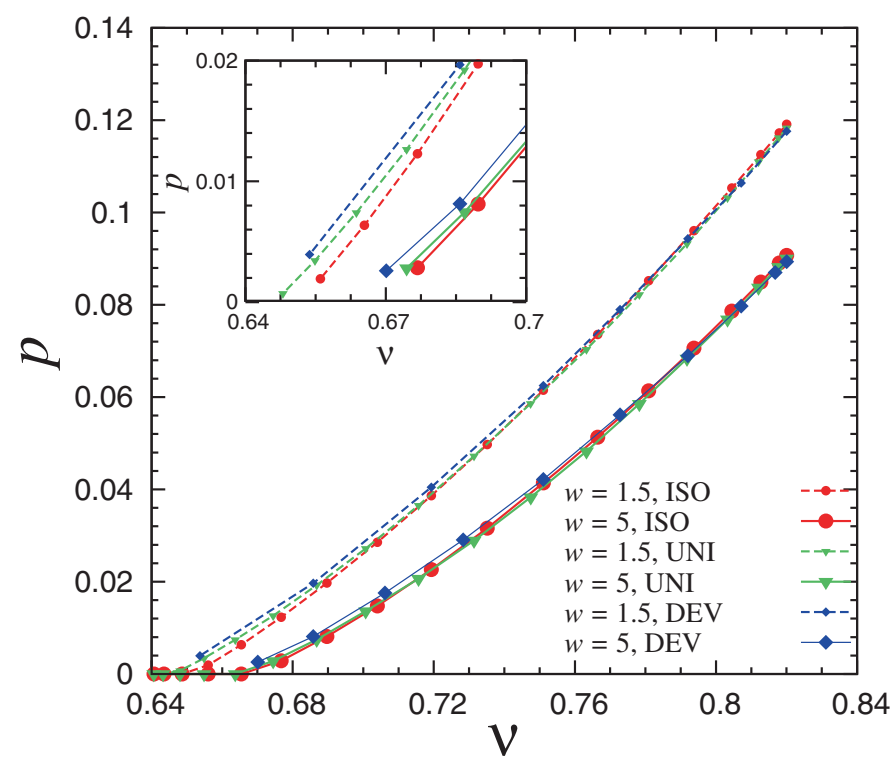

Figure 3.3: Evolution of non-dimensional pressure $p$ with volume fraction $v$ for the isotropic $(\bullet$, red), uniaxial $(\boldsymbol{\nabla}$, green) and deviatoric $(\checkmark$, blue) deformation modes, as shown in the legend. Small symbols represent $w=1.5$ and big symbols represent $w=5$. Inset is the zoomed-in area near the jamming volume fraction.

In Fig. 3.3, we plot the evolution of the non-dimensional pressure $p$ with volume fraction $v$ during isotropic, uniaxial and deviatoric deformation for polydispersities $w=1.5$ and 5 . Note that $p$ increases with $v$ starting from $v_{c}$, with slight differences related to different modes, as discussed in [84]. For a given volume fraction, we observe a decrease in the pressure with increasing polydispersity. A possible explanation for this feature is the distribution of the overlap $\delta(r) /\langle r\rangle$ as a function of the scaled particle radii $r_{\mathrm{sc}}$, as shown in Figs. 3.4a and $3.4 \mathrm{~b}$ for two volume fractions, $v=0.686$ and $v=0.82$. The particle radii are scaled such that $r_{\mathrm{sc}}=0$ and $r_{\mathrm{sc}}=1$ represent the smallest and largest particle in the configuration, respectively. A first observation is the unsurprising increase in the average overlap for all modes and polydispersities with increasing compression from $v=0.686$ in Fig. 3.4 a to $v=0.82$ in Fig. 3.4b, in agreement with Fig. 3.3. Based on this, and for linear contact model, we can claim that $P / k \propto \delta(r) /\langle r\rangle$, at least for small deformations. In addition, for two volume fractions shown, the overlap increases with increasing particle radii.

For both polydispersities, deviatoric deformation leads to the highest pressure, followed by the uniaxial and isotropic modes, respectively. This trend is clearly visible at lower volume fractions - as shown in the inset of Fig. 3.3, while for increasing volume fraction, the effect of the deformation mode reduces, as evident by the collapse of data in Fig. 3.4b. The agreement is confirmed by observing the average overlap $\langle\delta\rangle$ in Figs. 3.4a and 3.4b, with the data from uniaxial compression lying between the isotropic and deviatoric datasets. The trend observed 
in the evolution of the scaled pressure and distribution of the average overlaps are consistent with the fact that the isotropic and deviatoric modes are pure modes, while the uniaxial mode is a superposition of isotropic and deviatoric modes [128].

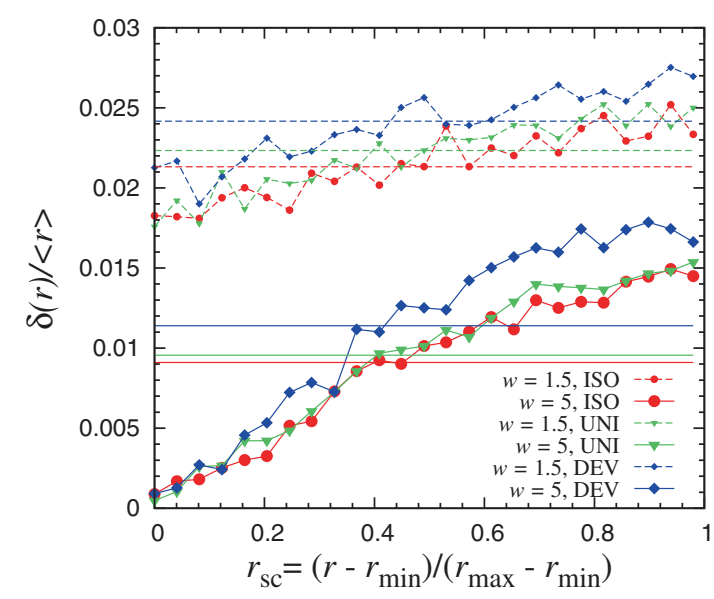

(a)

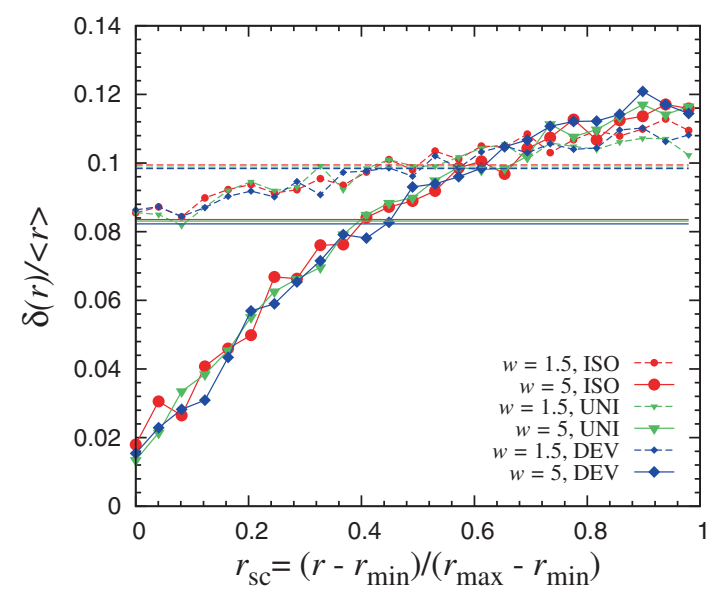

(b)

Figure 3.4: Average overlap $\delta$ per particle for a radius range scaled by average radius $\langle r\rangle$, plotted against a scaled radius axis $r_{\mathrm{sc}}=\left(r-r_{\min }\right) /\left(r_{\max }-r_{\min }\right)$ for the isotropic $(\bullet$, red $)$, uniaxial $(\boldsymbol{\nabla}$, green) and deviatoric $(\boldsymbol{\nabla}$, blue) deformation modes. Small symbols represent $w=1.5$ and big symbols represent $w=5$. Volume fractions are (a) $v=0.686$ (b) $v=0.82$. Solid and dashed horizontal lines are average overlap $\langle\delta\rangle$ in the system for the three modes for $w=1.5$ and $w=5$ respectively. Note that the $y$-axis range is different in the two plots.

Figs. 3.5 $(\mathrm{a}-\mathrm{c})$ show the effects of varying polydispersity on the scaled pressure in Eq. (3.10), where $p^{*}$ is plotted against volumetric strain $-\varepsilon_{v}$ for isotropic, uniaxial and deviatoric deformations. For a single deviatoric deformation the volume fraction is constant during the path and hence the pressure remains practically constant. In this work the data describing deviatoric mode will always refer to the values in the critical state, after large deformation (see [84] for more details), unless stated otherwise.

In the small strain region, for all deformation modes, the datasets collapse on each other. Only with increasing $-\varepsilon_{v}$, a small deviation of the simulation data is observed for the isotropic and deviatoric modes, due to the non-linear correction that shows up at large strain in Eq. (3.10). The analytical expression of the scaled pressure in Eq. (3.10) fits the simulation data well for all three deformation modes and polydispersity, in agreement with findings in $[69,70,84]$.

The collapse of data in $p^{*}$ for different polydispersity is explained by the fact that the relative distance from the jamming point $v_{c}$ is the factor that isolates the effect of pressure $p$ and the contact network $F_{\mathrm{v}} / g_{3}=v C$, leading to same $p^{*}$ for the same $-\varepsilon_{v}$, i.e., same scaled pressure for the same distance from the corresponding jamming point. The fit parameters for $p^{*}$ 


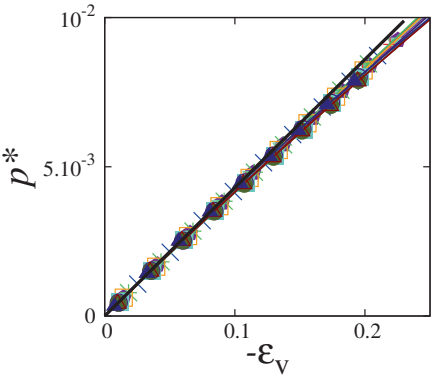

(a)

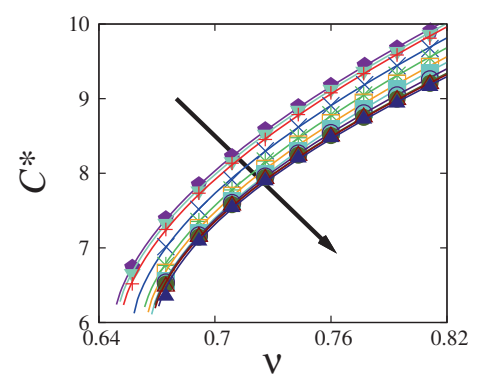

(d)

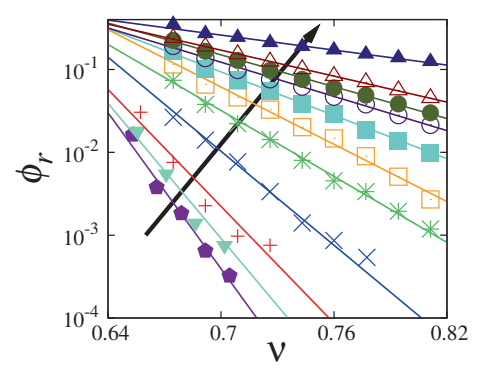

(g)

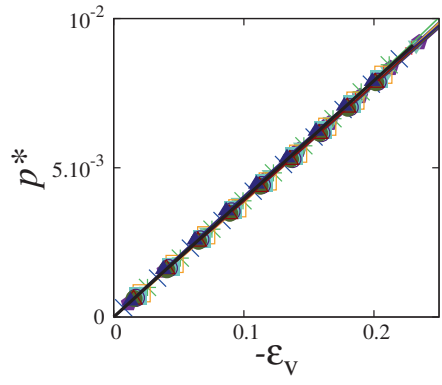

(b)

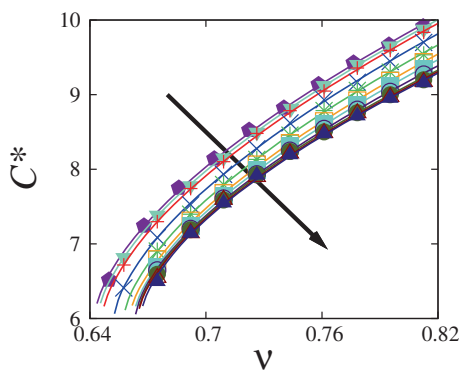

(e)

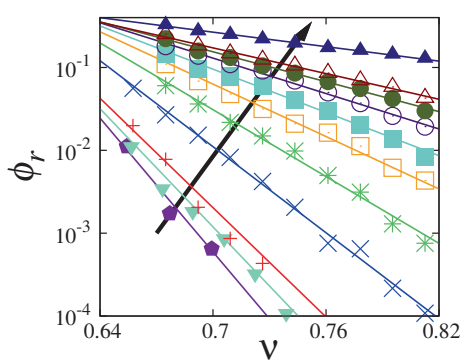

(h)

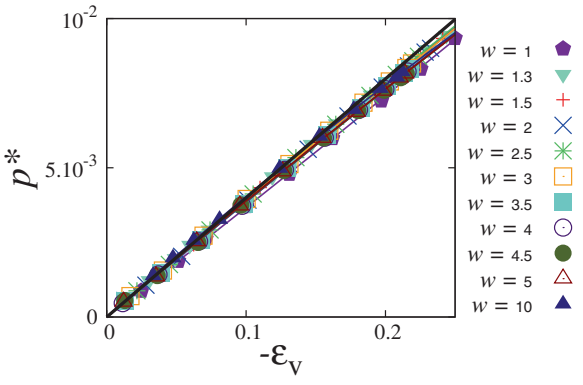

(c)

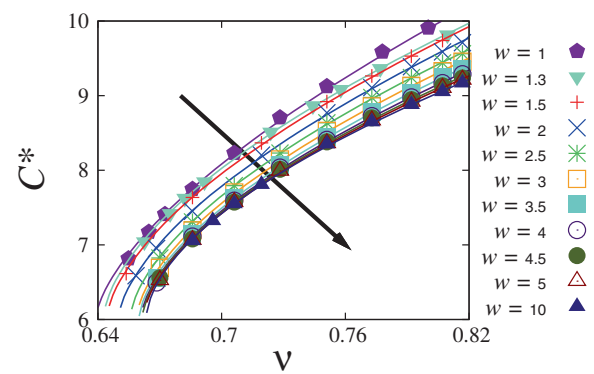

(f)

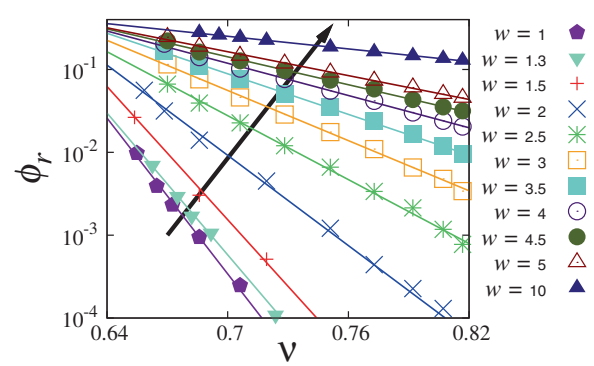

(i)

Figure 3.5: Effect of polydispersity $w$ on scaled pressure $p^{*}$, coordination number $C^{*}$, and fraction of rattlers $\phi_{r}$ for the three deformation modes namely, isotropic compression (first column), uniaxial compression (middle column) and deviatoric deformation (right column). The solid lines are the fits to the corresponding macroscopic properties using Eqs. (3.10), (3.12) with $C_{0}=6, \alpha=0.60$ for the three modes, and (3.13). The arrows indicate the increasing polydispersity. The solid black line in the $p^{*}$ plot is Eq. (3.10) without the nonlinear term. All the fit parameters are presented in Table 3.4 in the appendix.

are given in Fig. 3.6 (and Table 3.4 in the appendix). The parameter $p_{0}$ is fairly constant with increasing polydispersity, with $p_{0}$ values higher for the isotropic case and uniaxial and deviatoric $p_{0}$ being very close. This is in agreement with expectations, as in both uniaxial and deviatoric deformations, anisotropy develops along the path, shear leading to a reduction of jamming volume fraction, and the value of the non-dimensional pressure is increasing with respect to the (pure) isotropic case. All this leads to slightly reduced $v_{c}$ for the sheared 


\begin{tabular}{c|cc}
\hline Mode & $v_{c}^{0}$ & $v_{c}^{\infty}$ \\
\hline ISO & 0.6453 & 0.6710 \\
UNI & 0.6394 & 0.6675 \\
DEV & 0.6381 & 0.6647 \\
{$[147]$} & 0.65 & 0.6828 \\
\hline
\end{tabular}

Table 3.2: Summary of parameters $v_{c}^{0}$ and $v_{c}^{\infty}$ using Eq. (3.11) presented in Fig. 3.7, for the isotropic, uniaxial and deviatoric deformation modes.

mode, relative to the isotropic mode. The non-linear contribution from $\gamma_{p}$ fluctuates for smaller polydispersity and becomes significant for higher $w$.

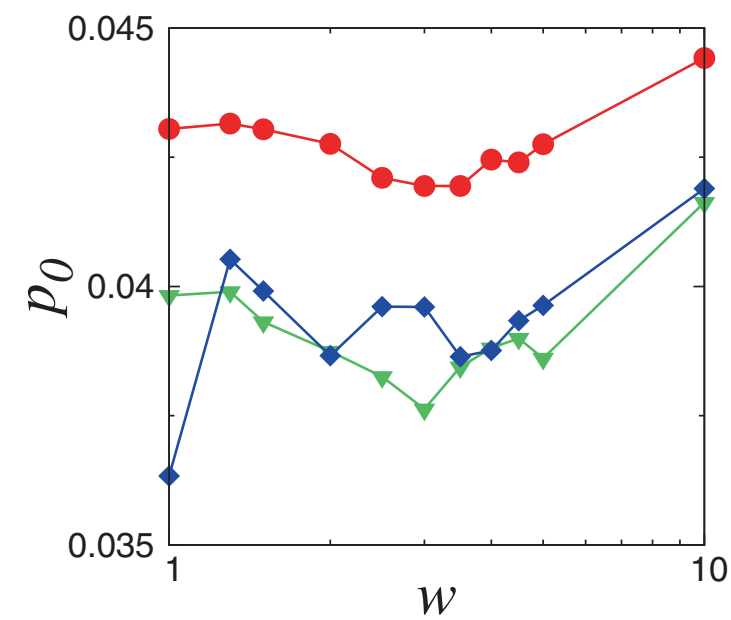

(a)

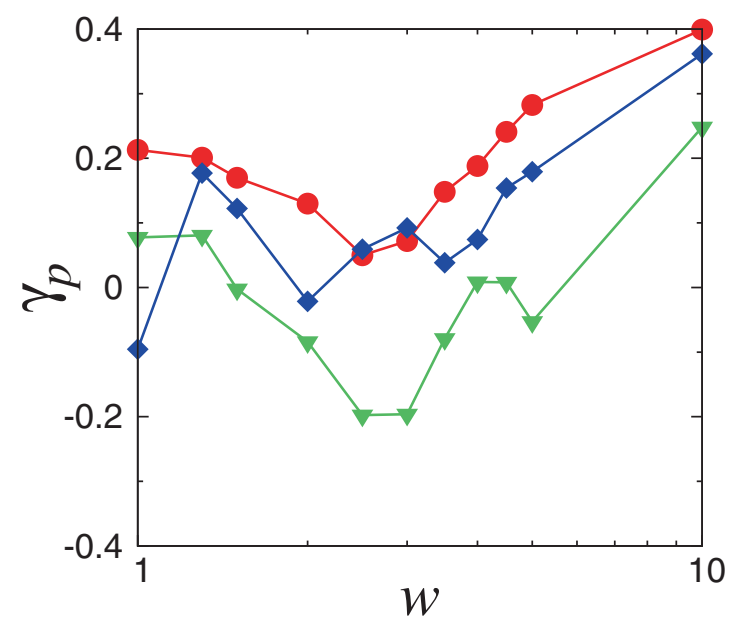

(b)

Figure 3.6: Comparison of the fit parameters (a) $p_{0}$ and (b) $\gamma_{p}$ with polydispersity $w$ for the analytical equations of scaled pressure $p^{*}$ using Eq. (3.10) for the isotropic (•, red), uniaxial $(\boldsymbol{\nabla}$, green) and deviatoric $(\boldsymbol{\nabla}$, blue) deformation modes. The fit parameters are presented in Table 3.4 in the appendix.

From the analysis of the pressure behavior by fitting Eq. (3.10), we can extract the dependence of the jamming volume fraction $v_{c}$ on the polydispersity $w$ and the deformation mode, as shown in Fig. 3.7. The jamming volume fraction increases with increasing polydispersity, with $v_{c}$ for the isotropic case giving highest values. The $v_{c}$ law for the 'mixed' uniaxial mode is bordered on both sides by the isotropic and deviatoric datasets. This is consistent with findings in [84] where $v_{c}^{\mathrm{ISO}}>v_{c}^{\mathrm{UNI}}>v_{c}^{\mathrm{DEV}}$. In this case a similar argument holds as mentioned for $p_{0}$, related to developing anisotropy during the over-compression, that explains the trend of the jamming point between isotropic, deviatoric and uniaxial. This confirms that the jam- 


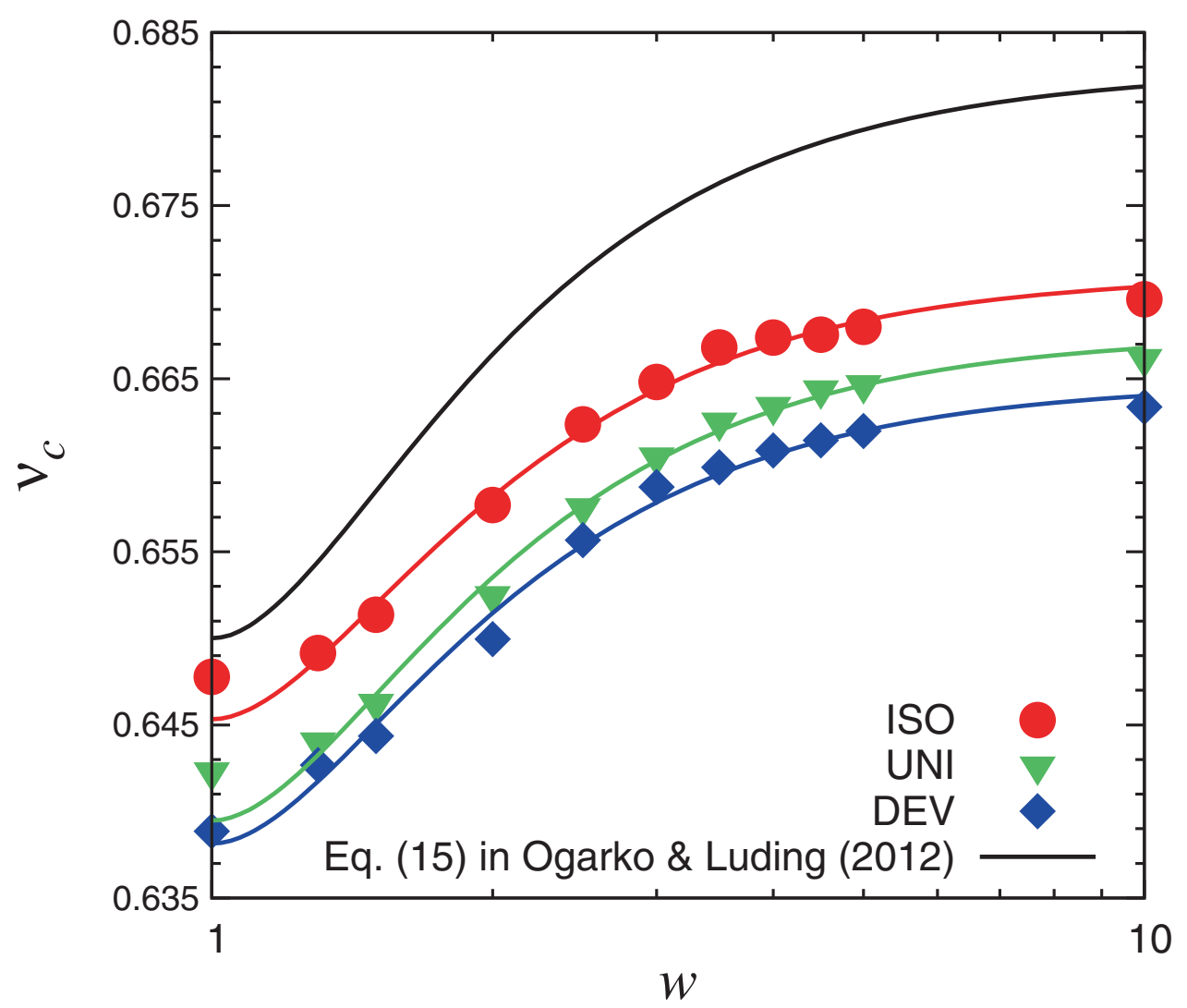

Figure 3.7: Evolution of jamming point $v_{c}$ with polydispersity $w$ for the deformation modes considered. Corresponding solid lines are the theoretical predictions for different modes using Eq. (3.11). Note that the fit is applied only to $w>1.2$, since local crystallization $[147,175]$ might happen at lower polydispersity causing $v_{c}$ values much higher than the disordered, random prediction.

ming volume fraction is not a single value but depends on the deformation history of the packing.

A theoretical prediction for the variation in $v_{c}$ under isotropic compression of polydisperse hard spheres is presented by [147]:

$$
v_{c}(w)=v_{c}^{\infty}-\left(v_{c}^{\infty}-v_{c}^{0}\right)\left(3 w^{-2}-2 w^{-3}\right),
$$

where $v_{c}^{0}$ and $v_{c}^{\infty}$ are the jamming volume fractions for $w=1$ and $w \rightarrow \infty$ respectively. We apply Eq. (3.11) to the three deformation modes, and in Fig. 3.7 we show the prediction using for hard spheres together with the $v_{c}$ values for the three modes, and the fitting curves, where the parameters $v_{c}^{0}$ and $v_{c}^{\infty}$ are presented in Table 3.2. Besides the quantitative disagreement due to the difference between hard and soft spheres, both systems show a very similar trend, the predictions working well for all the three modes. The simulations in [147], leading to Eq. (3.11), were carried out by very slow isotropic compression from the low density collisional regime, where the fluctuation velocities were not relaxed as done in this study. The strong 
kinetic energy fluctuations represent a type of 'tapping' that allows the system to relax to better packed configurations with larger $v_{c}$. The data in Fig. 3.7 from [147] thus represents an upper limit of optimal compaction, which is not reached by e.g. slow over-compression to $v_{\max }=0.82$. Eq. (3.11) can then capture the evolution of $v_{c}$ with polydispersity, irrespective of the deformation modes, when the fit parameters are properly defined. This interesting feature shows that $v_{c}$ acts as a state variable, able to describe the configuration of the assembly and thus represent its history, as also reflected by the overlaps, see Fig. 3.4.

\subsubsection{Coordination Number}

It has been shown in $[69,84]$ that under isotropic deformation, the corrected coordination number, $C^{*}$ follows the power law:

$$
C^{*}(v)=C_{0}+C_{1}\left(\frac{v}{v_{c}}-1\right)^{\alpha},
$$

where $C_{0}=6$ is the isostatic value in the frictionless case. $\alpha$ and $C_{1}$ are fit parameters, while we use $v_{c}$ from $p^{*}$ extrapolation analysis as input value, leading to one less fit parameter for $C^{*}$. We observe a very small variation (3\%) of $\alpha$ with polydispersity and deformation modes [84] but for simplicity set it to a fixed value of 0.60 in this work [159]. Only $C_{1}$ is then the residual free fit parameter.

In Figs. 3.5(d-f), we compare the evolution of the corrected coordination number $C^{*}$ as a function of volume fraction $v$ during isotropic, uniaxial and after deviatoric loading and show its dependence on polydispersity. The behavior is qualitatively similar for all the three deformation paths: contacts close and the coordination number increases with increasing volume fraction. Moreover, for the three modes, configurations with lower polydispersity result in a higher number of contacts per particle. The data are well fitted by Eq. (3.12) with the fit parameter $C_{1}$ as function of $w$ shown in Fig. 3.8a. A systematic decrease in $C_{1}$ is observed with increasing polydispersity. The $C_{1}$ values of the 'mixed' uniaxial mode lie between the isotropic and deviatoric dataset.

Further increase in the polydispersity beyond $w=4.5$ did not lead to a further change of $C_{1}$ - evidenced by the collapse of the $C^{*}$ lines on each other. This suggests that uniform size polydispersity influences the micromechanics only within a certain limit. For highly polydisperse packings $(w>5)$, the limit is approached because the critical volume fraction $v_{c}$ saturates (see Fig. 3.7).

In order to further investigate the behavior of the coordination number $C^{*}$ we study the distribution of contacts per particle radius fraction. In Figs. 3.9a and 3.9b, we plot the average number of contacts (excluding the rattlers) for a radius range, defined as $C^{*}(r)$, versus the scaled radius $r_{\mathrm{sc}}=\left(r-r_{\min }\right) /\left(r_{\max }-r_{\min }\right)$ for $v=0.686$ and $v=0.82$ for the three deformation modes. $C^{*}(r)$ increases with increasing $r_{\mathrm{sc}}$ for all the three modes, that is the number 


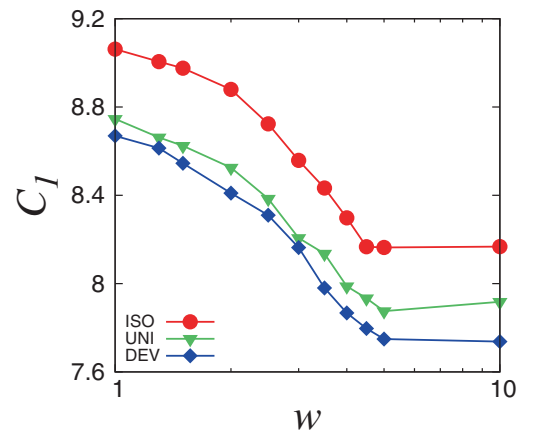

(a)

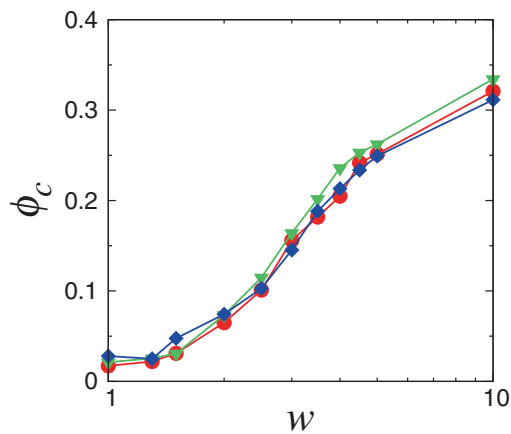

(b)

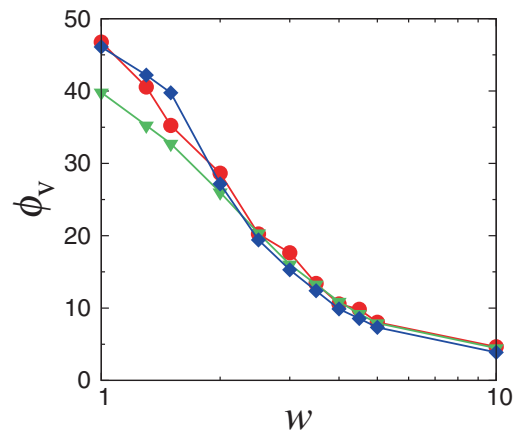

(c)

Figure 3.8: Comparison of the fit parameters for the analytical equations of coordination number $C^{*}$ and rattler fraction $\phi_{r}$ using Eqs. (3.12) with $C_{0}=6, \alpha=0.60$, and (3.13), respectively, for the isotropic $(\bullet$, red), uniaxial $(\boldsymbol{\nabla}$, green) and deviatoric $(\boldsymbol{\vee}$, blue) deformation modes. (a) Effect of polydispersity $w$ on coordination number $C^{*}$ fit parameters : $C_{1}$. $(\mathrm{b}-\mathrm{c})$ Effect of polydispersity $w$ on rattler fraction $\phi_{r}$ fit parameters : $\phi_{c}$ and $\phi_{\mathrm{v}}$. The fit parameters are presented in Table 3.4 in the appendix.

of contacts is bigger for bigger particles. This is expected because the bigger particles have larger surface area and thus can be in contact with more particles. A similar argument explains the relation between the particle coordination number $C^{*}(r)$ and size dependence on polydispersity: smaller $w$ leads to higher number of contacts for the smallest particles and to a weaker variation of $C^{*}(r)$ with $r_{\mathrm{sc}}$. The crossover radius $r_{\mathrm{sc}}$ where different systems have same $C^{*}(r)$ value, shifts towards the left for higher volume fractions. As expected, for higher volume fractions, $C^{*}(r)$ increases with $r_{\mathrm{sc}}$, as shown in Fig. 3.9b, since more contacts are formed as the volume of the box becomes smaller. Comparing the deformation modes, only very minimal differences appear, visible for low volume fraction, $v=0.686$, as shown in Fig. 3.9a and negligible for high volume fraction, $v=0.82$, as seen in Fig. 3.9b. The average values for isotropic deformation are smaller, larger for deviatoric, and the mixed uniaxial deformation mode lies in between the two [84].

\subsubsection{Fraction of rattlers}

The analytical expression for the fraction of rattlers is proposed in $[69,84]$ as

$$
\phi_{r}(v)=\phi_{c} \exp \left[-\phi_{\mathrm{v}}\left(\frac{v}{v_{c}}-1\right)\right]
$$

where $\phi_{c}$ and $\phi_{\mathrm{v}}$ are fit parameters, and $v_{c}$ is the jamming volume fraction inferred from Eq. (3.10) for the different deformation modes. We show the effect of polydispersity on the fraction of rattlers under isotropic, uniaxial and deviatoric deformation in Figs. 3.5 $(\mathrm{g}$ - i) and the fit parameters variation with $w$ in Figs. 3.8b and 3.8c. A first observation is that the fraction of rattlers decreases exponentially with increasing volume fraction [84] in 


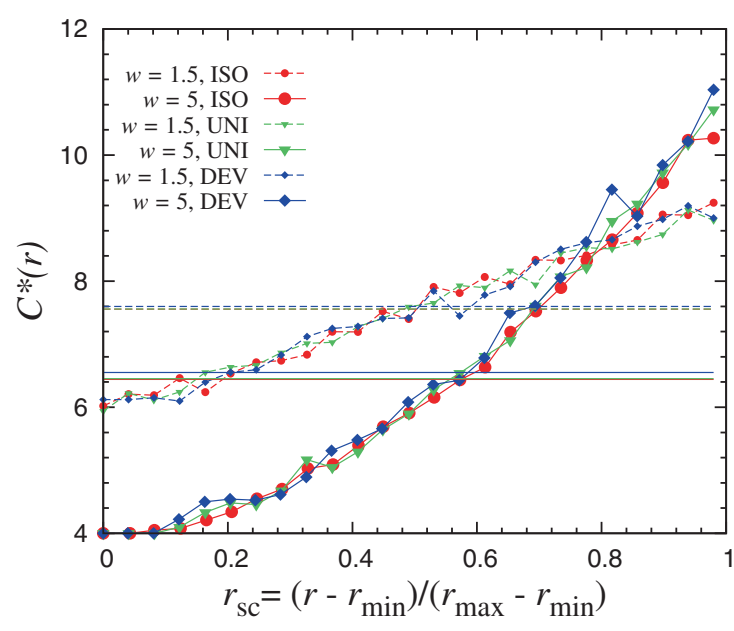

(a)

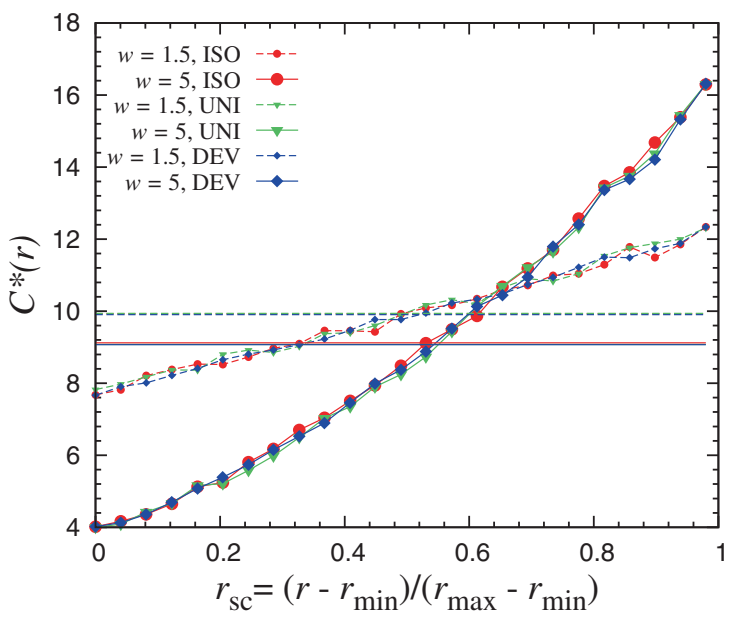

(b)

Figure 3.9: Average contacts per particle excluding the rattlers $C^{*}(r)$ for a radius range, plotted against a scaled radius $r_{\mathrm{sc}}=\left(r-r_{\min }\right) /\left(r_{\max }-r_{\min }\right)$ for the isotropic $(\bullet$, red $)$, uniaxial $(\boldsymbol{\nabla}$, green) and deviatoric $(\checkmark$, blue) deformation modes. Small symbols represent $w=1.5$ and big symbols represent $w=5$. Volume fractions are (a) $v=0.686$, and (b) $v=0.82$. Solid and dashed horizontal lines are the average coordination numbers $\left\langle C^{*}(r)\right\rangle=C^{*}$ in the system for the three modes, for $w=1.5$ and $w=5$ respectively. The $y$-axis range is different in the two plots.

agreement with Eq. (3.13). Furthermore, the increase of polydispersity leads to an increase of the fraction of rattlers in the system. This is not surprising since the volume occupied by finer/smaller particles is smaller in highly polydisperse systems. Contacts of these smaller particles are transient since they have more freedom to move within the system (for this size distribution - not in general). In some cases, they may become 'caged' between larger particles without having sufficient (four or more) contacts with their neighbors. This leads to a drop in the coordination number and an increase in the fraction of rattlers.

Also interesting is the evolution of the parameters of Eq. (3.13): $\phi_{c}$ (initial point) and $\phi_{\mathrm{v}}$ which represents the slope. A systematic increase in $\phi_{c}$ with increasing polydispersity is observed, whereas the slope $\phi_{\mathrm{v}}$ decreases with increasing polydispersity. This indicates that even though the fraction of rattlers in highly polydisperse systems is higher, the rate at which rattlers are lost in these systems during compression decreases with $w$. This again is related with the 'cage' argument, as very small particles are caged by big particles and need a high compression degree to gain new contacts with respect to medium sized particles (see Figs. 3.9a and 3.9b). Interestingly, both parameters $\phi_{c}$ and $\phi_{\mathrm{v}}$, as presented in Fig. 3.8, are seemingly unaffected by the deformation mode, stating that the history of the sample can be fully represented by $v_{c}$, when the rattler fraction is analyzed.

Finally, we plot in Fig. 3.10 the evolution of the isotropic fabric $F_{\mathrm{v}}=g_{3} v C$ versus volume 


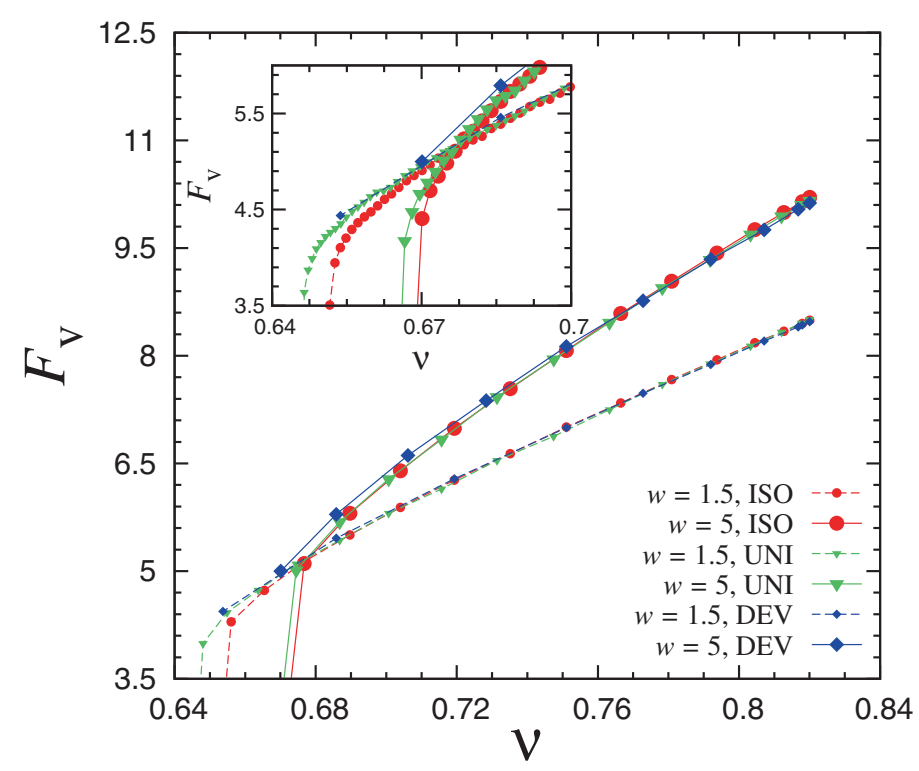

Figure 3.10: Evolution of isotropic fabric $F_{\mathrm{v}}$ with volume fraction $v$ for the isotropic $(\bullet$, red), uniaxial ( $\boldsymbol{\nabla}$, green) and deviatoric $(\checkmark$, blue) deformation modes as shown in the legend. Small symbols represent $w=1.5$ and big symbols represent $w=5$. Inset is the zoomed-in area near the jamming volume fractions.

fraction during isotropic compression. $F_{\mathrm{v}}$ increases with volume fraction and polydispersity $w$ and shows a trend opposite with respect to the corrected coordination number $C^{*}$ in Figs. $3.5(\mathrm{~d}-\mathrm{f})$. This can be explained by observing the rattlers: when particles with less than four contacts are included in the calculation of $C$, the values of $F_{\mathrm{v}}$ grow with increasing $w$ and $v$. For both polydispersities, near the jamming volume fraction, deviatoric deformation has the highest $F_{\mathrm{v}}$, isotropic deformation has the lowest and the mixed uniaxial mode is in between the two - as shown in the inset of Fig. 3.10. This variations disappear for large volume fractions. However, the differences between the three modes for $F_{\mathrm{v}}$ are smaller compared to the isotropic part of stress $p$, as it is related to small differences in the average contact number per particle, as shown in Figs. 3.9a and 3.9b.

\subsubsection{Effect of polydispersity on deviatoric quantities}

In this section, we present the effects of polydispersity on the evolution of deviatoric stress and deviatoric fabric during uniaxial and deviatoric deformation paths. The former, a macroscopic property quantifies the stress anisotropy [84], while the latter is a microscopic property related to the structural anisotropy of the contact network. Here, we focus on the simulation results for the uniaxial and deviatoric deformation datasets (since the deviatoric quantities are only fluctuating around zero for the isotropic mode). Later in section 3.5, we will use the information obtained from the above mentioned quantities to calibrate a constitutive model, using isotropic information and the deviatoric test. In the end, we will test the predictive power of the calibrated constitutive model on an independent uniaxial compression 
test.

\subsubsection{Deviatoric stress}

In Fig. 3.11a, we plot the deviatoric stress ratio $\left(s_{\mathrm{dev}}=\sigma_{\mathrm{dev}} / P\right)$ as a function of deviatoric strain $\varepsilon_{\mathrm{dev}}$ during deviatoric deformation for packings with three different polydispersities. The volume fraction $v$ is 0.751 in all cases, and stays constant during the numeric experiments. The deviatoric stress grows initially with rate $\beta_{s}$ from random initial values (note the small random initial anisotropy present in each sample) until an asymptote, $s_{\mathrm{dev}}^{\max }$ at steady state is reached, where it remains fairly constant, in agreement to what is reported in $[41,84,104,118]$. The steady state value increases with polydispersity (the highly fluctuating values are in the range $0.11 \pm 0.02,0.12 \pm 0.03$ and $0.15 \pm 0.035$ for $w=1.5,2$ and 5 , respectively). Surprisingly, while the deviatoric stress $\sigma_{\mathrm{dev}}$ is practically unaffected by $w$, the pressure $P$ decreases with increasing polydispersity (see Fig. 3.3), leading to the dependence of the ratio $\sigma_{\mathrm{dev}} / P$ on $w$ as observed. On the other hand, the slope $\beta_{s}$, proportional to the shear stiffness (scaled by pressure) of the initial isotropic configurations, is a function of the isotropic fabric $F_{\mathrm{v}}$, as shown in the inset of Fig. 3.11a. The relation between isotropic fabric and polydispersity, extensively discussed in [69] and reported in Fig. 3.10, makes $\beta_{s}$ a decreasing function of $w$.

Furthermore, in Fig. 3.12a we plot the deviatoric stress as a function of deviatoric strain during uniaxial compression, for packings with different polydispersity $w=1.5,2$ and 5 . The uniaxial test starts from initial volume fraction $v_{i}=0.72$ (the same value used for the previous deviatoric simulations), and reaches the maximum volume fraction $v_{\max }=0.82$. As for the deviatoric simulations, higher polydispersity leads to higher $s_{\mathrm{dev}}^{\max }$ at steady state also for the uniaxial deformations. The same argument about the dependence of pressure on polydispersity holds and explains the behavior in Fig. 3.12a. We observe larger fluctuations for the uniaxial deformation mode with respect to the deviatoric one, with averages and errors $s_{\mathrm{dev}}^{\max } \approx 0.10 \pm 0.025,0.11 \pm 0.035$ and $0.15 \pm 0.04$ for $w=1.5,2$ and 5 , respectively. We relate the increasing fluctuations to the non-conserved volume [84] and more "violent" rearrangements. Note that different sign conventions are used in Eq. (3.6) to calculate the deviatoric stress for deviatoric and uniaxial simulations, since the definition of the sign function Fsgn depends on the deformation mode, as discussed in section 3.2, i.e. the strain eigen-system. Since the latter is parallel to $x, y, z$, the sign function for uniaxial compression (negative strain components versus positive stress and fabric) is

$$
\operatorname{Fsgn}(\mathbf{Q})=\operatorname{sgn}\left(Q_{z z}-0.5\left(Q_{x x}+Q_{y y}\right)\right)
$$

where the $z$-wall is moving and the $x$ - and $y$-walls are not. For deviatoric deformation

$$
\operatorname{Fsgn}(\mathbf{Q})=\operatorname{sgn}\left(Q_{y y}-Q_{x x}\right),
$$

with $x$-wall expanding, $y$ - compressing and a non-mobile $z$-wall. The sign convention explains the random initial values for the same initial packings in Figs. 3.11a and 3.12a. 


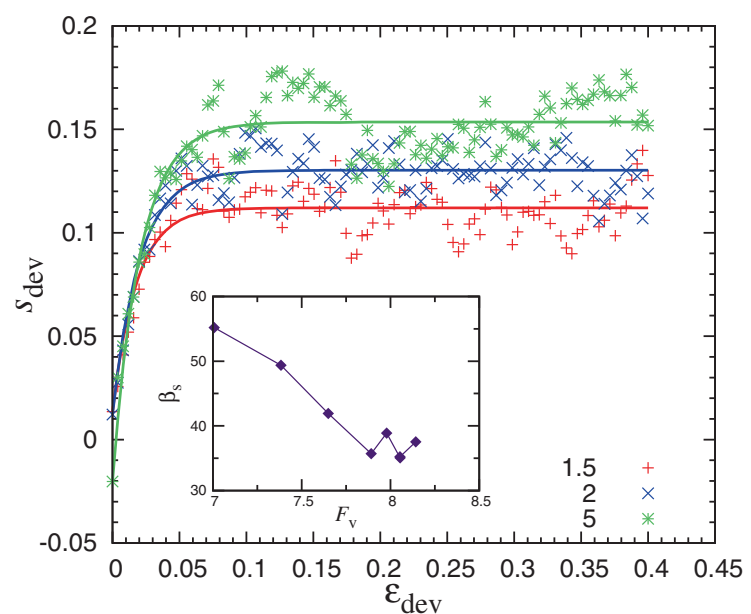

(a)

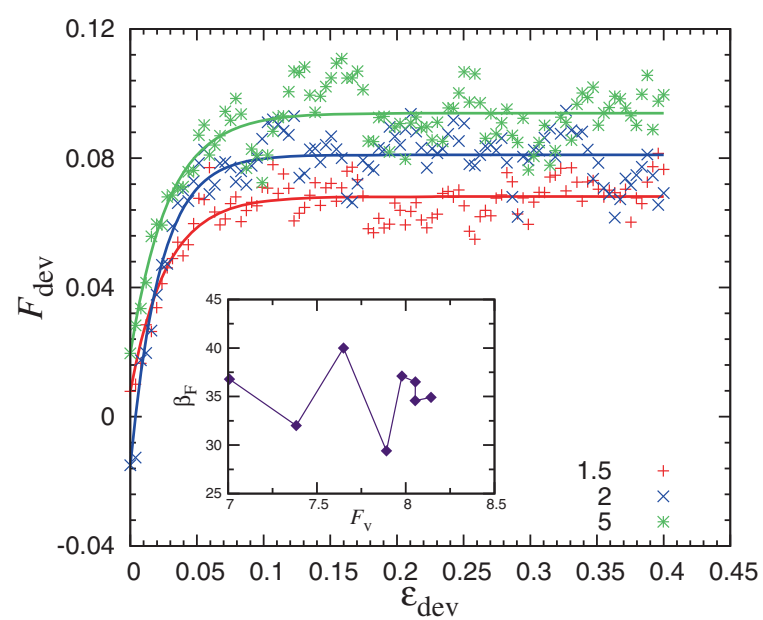

(b)

Figure 3.11: (a) Deviatoric stress ratio $s_{\mathrm{dev}}=\sigma_{\mathrm{dev}} / P$ plotted against deviatoric strain from the deviatoric (volume conserving) mode for three polydispersities $w=1.5,2$ and 5 as shown in the legend. The data points are the simulation results while the solid lines through them represent fits to the data using Eq. (3.15). The volume fraction is $v=0.75$. (b) Deviatoric fabric $F_{\text {dev }}$ plotted against deviatoric strain for the same cases as in (a). The data points are simulation results while the solid lines through them are fits to the data using Eq. (3.16). The corresponding inset shows the behavior of growth rates $\beta_{s}$ and $\beta_{F}$ with isotropic fabric $F_{\mathrm{V}}$ for different $w$.

\subsubsection{Effect on deviatoric Fabric}

The evolution of the deviatoric fabric, $F_{\mathrm{dev}}$ as a function of the deviatoric strain $\varepsilon_{\mathrm{dev}}$ is shown in Fig. 3.11b for the same deviatoric simulations as above. $F_{\mathrm{dev}}$ builds up from different random (small) initial values with rate $\beta_{F}$ to different saturation values $F_{\mathrm{dev}}^{\max }$. Interestingly, the slope $\beta_{F}$ seems to be constant (besides large fluctuations), irrespective of different polydispersity of the initial configurations. This is surprising, as the initial samples have different contact network density $F_{\mathrm{v}}$, due to polydispersity, and the incremental response of fabric for isotropic samples is known to depend on the isotropic value $F_{\mathrm{v}}$ as state variable [104]. The critical value $F_{\mathrm{dev}}^{\max }$ shows a different trend from $\beta_{F}$, but similar to $s_{\mathrm{dev}}^{\max }$ as it increases with polydispersity $w$. This is reasonable, when we think of the kinematics at small scale: particles with a large difference in size have more freedom to rearrange and modify the contact network during compression. The behavior of $F_{\mathrm{dev}}^{\max }$ is consistent with the decrease of $C^{*}$ in Figs. 3.5(e-f), as a lower coordination number is usually associated with a higher anisotropy.

In order to further investigate the anisotropic behavior of the material, we study the deviatoric fabric $F_{\mathrm{dev}}(r)$ per particle radius for the volume conserving deviatoric tests focusing on the large shear strain configurations, i.e. $\varepsilon_{\mathrm{dev}}=0.40$. To account only for the orientation but 


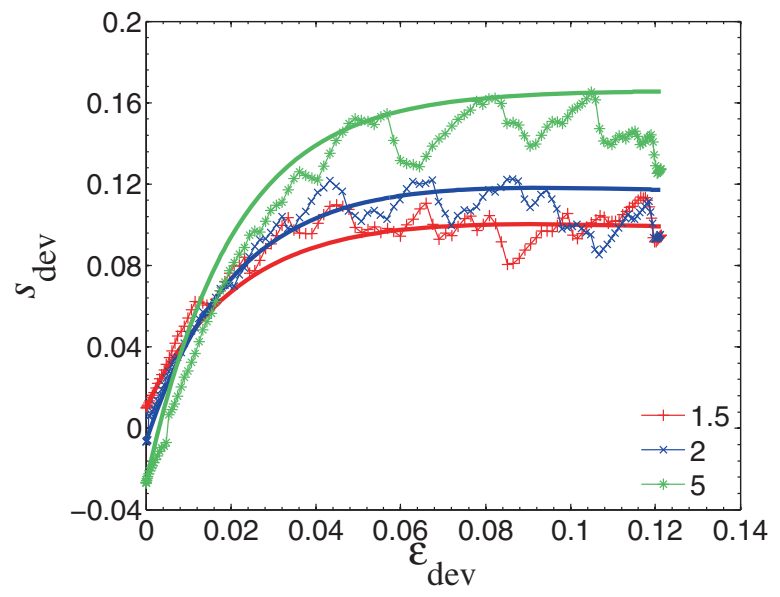

(a)

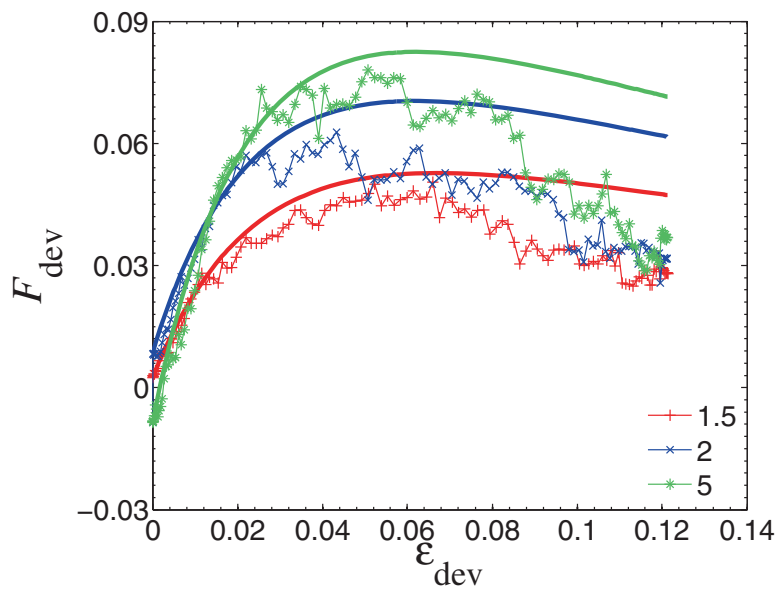

(b)

Figure 3.12: (a) Deviatoric stress ratio $s_{\mathrm{dev}}=\sigma_{\mathrm{dev}} / P$ plotted against deviatoric strain from the uniaxial mode for three polydispersities $w=1.5,2$ and 5 as shown in the inset. The data points are the simulation results while the solid lines through them represent a prediction to the data using Eq. (3.15). The starting volume fraction is $v_{i}=0.72$ and the maximum volume fraction is $v=0.82$ for all the cases of polydispersity. (b) Deviatoric fabric $F_{\mathrm{dev}}$ plotted against deviatoric strain for the same cases as in (a). The data points are simulation results while the solid lines through them are the prediction using Eq. (3.16).

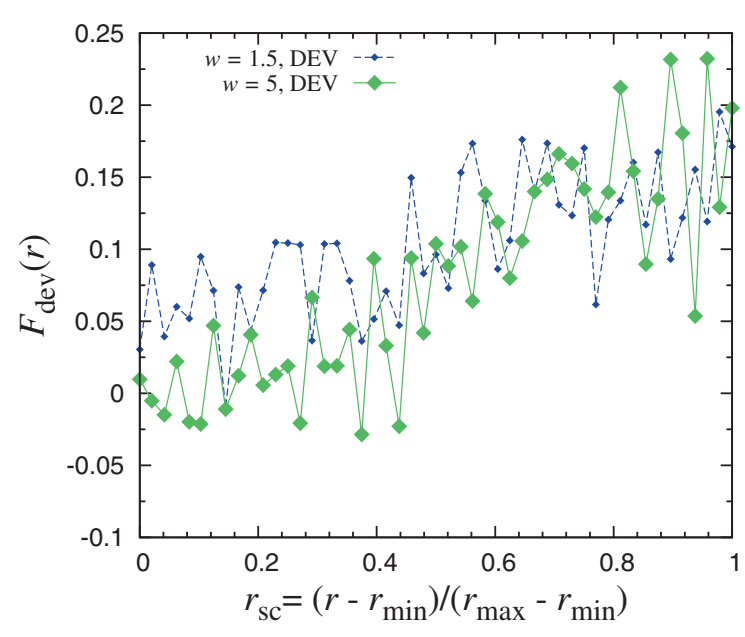

(a)

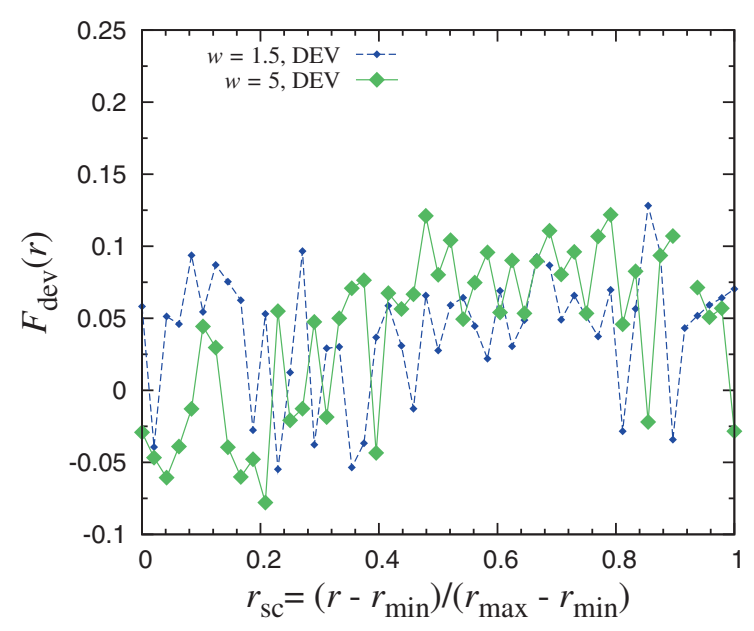

(b)

Figure 3.13: Deviatoric fabric per particle radius fraction $F_{\mathrm{dev}}(r)$, plotted against a scaled radius $r_{\mathrm{sc}}=\left(r-r_{\min }\right) /\left(r_{\max }-r_{\min }\right)$ for the deviatoric deformation mode, after large shear strain $\varepsilon_{\mathrm{dev}}=0.40$. Small symbols represent $w=1.5$ and big symbols represent $w=5$. Volume fractions are (a) $v=0.686$, and (b) $v=0.82$. 
not for the particle radii, we modify Eq. (3.3) such that during the binning process, the particle volume is not taken into account and to make this quantity bin independent. First, we calculate the $F_{\mathrm{dev}}$ for every particle in the system and then to calculate the overall $F_{\mathrm{dev}}(r)$ of a bin, the $F_{\mathrm{dev}}$ is scaled by the ratio of total volume of particle in that bin divided by the total particle volume in the system. In Figs. 3.13a and 3.13b, we plot $F_{\mathrm{dev}}(r)$ versus the scaled radius $r_{\mathrm{sc}}=\left(r-r_{\min }\right) /\left(r_{\max }-r_{\min }\right)$ for $v=0.686$ and $v=0.82 . F_{\mathrm{dev}}(r)$ increases with increasing $r_{\mathrm{sc}}$, meaning that the bigger particles form a sub-network, whose orientation follows the applied shear strain. These are the particles that belong to the force chains [161] and carry the majority of the applied load. On the other hand, $F_{\mathrm{dev}}(r)$ is small for small $r_{\mathrm{sc}}$, as the small particles arrange randomly, i.e. isotropically and 'caged' in the voids among the bigger particles, as already mentioned in section 3.4.2.2. However, the differences in $F_{\mathrm{dev}}(r)$ due to polydispersity (at high $r_{\mathrm{sc}}$ ) disappears in case of dense sample, as the volume of the box becomes smaller, and the particles have less freedom to align with the applied deformation leading to a smaller $F_{\mathrm{dev}}(r)$ when compared with a loose system (within the rather large fluctuations).

The evolution of the deviatoric fabric under uniaxial deformation is presented in Figure $3.12 \mathrm{~b}$ for different polydispersity. In a similar fashion to deviatoric stress ratio, $F_{\mathrm{dev}}$ builds up from different (random, but small) initial values and reaches different maxima for different polydispersity, with $w=5$ showing the highest peak, while the slope $\beta_{F}$ stays unaffected by $w$. For larger strain, the structural anisotropy decreases rapidly towards zero (data not shown). This indicates that more new contacts are created in the axial direction compared to the perpendicular isotropic plane at the beginning of the loading path. At higher deviatoric strain [85], the fabric behavior becomes opposite and now more new contacts are created in the horizontal direction rather then in the vertical one, where most available neighbors already have come into contact. The 'softening' in deviatoric fabric does not correspond to any decrease in deviatoric stress that grows monotonically until saturation is reached (see Fig. 3.12a). The origin of this interesting feature in the uniaxial simulation, where stress and fabric show non-colinearity, and the strain eigen-system is prescribed by the wall motion, will be presented elsewhere.

\subsection{Calibration of the continuum model and prediction}

In this section, we will present the microscopic simulation results with a short review of an anisotropy continuum model as introduced in [128]. We will calibrate the free parameters in the model as function of polydispersity $w$ and volume fraction $v$, using the isotropic and purely deviatoric deformation experiments. Finally, using the model, a prediction of an independent test, i.e. the uniaxial deformation mode will be presented. 


\subsubsection{Reduced theoretical model}

Most standard constitutive models with wide application fields, like elasticity, elasto-plasticity, or fluid-/gas-models of various kinds, were applied also to granular flows - sometimes with success, but typically only in a very limited range of parameters and flow conditions; for overviews see $[16,52,70,84,88,128,140,194]$. While most of these theories can be and some have been extended to accommodate anisotropy of the microstructure, only very few models account for an independent evolution of the microstructure as for example $[67,128,190]$.

We use the constitutive model, as proposed in [128], generalized for a $\mathcal{D}$-dimensional system:

$$
\begin{aligned}
\delta P & =\mathcal{D} B \delta \varepsilon_{\mathrm{v}}+A S \delta \varepsilon_{\mathrm{dev}} \\
\delta \sigma_{\mathrm{dev}} & =\mathcal{D} A \delta \varepsilon_{\mathrm{v}}+G^{\mathrm{oct}} S \delta \varepsilon_{\mathrm{dev}} \\
\delta A & =\beta_{A} \operatorname{sign}\left(\delta \varepsilon_{\mathrm{dev}}\right)\left(A^{\mathrm{max}}-A\right) \delta \varepsilon_{\mathrm{dev}}
\end{aligned}
$$

The model involves three moduli, namely, the classical bulk modulus $B$ [69], the octahedral shear modulus $G^{\text {oct }}$, and the "anisotropy modulus" $A$. Due to the modulus $A$, the model provides a cross coupling between the two types of stress and strain in the model, namely the hydrostatic and the shear (deviatoric) stresses react to both isotropic and deviatoric strains. $S=\left(1-s_{\mathrm{dev}} / s_{\mathrm{dev}}^{\max }\right)$ is an abbreviation for the stress isotropy with the stress ratio $s_{\mathrm{dev}}$ already introduced in section 3.4.3. The parameter $s_{\mathrm{dev}}^{\max }$ resembles the macroscopic friction (depending on our definition, $s_{\mathrm{dev}}=3 q=3 \sin \varphi$, where $q$ is the shear stress ratio and $\varphi$ is the internal friction angle as in [10] and others) while $\beta_{s}$ is the growth rate of $s_{\mathrm{dev}}$. The parameter $A^{\mathrm{max}}$ in the evolution equation of $A$ represents the maximum anisotropy that can be reached at saturation, and $\beta_{A}=\beta_{F}$ determines how fast the asymptote is reached (growth rate) when a material is subjected to deviatoric strain $\varepsilon_{\operatorname{dev}}$ [84]. Both $A^{\max }$ and $\beta_{A}$ are model parameters and can be extracted from fits to the macroscopic simulation results. In a nutshell, the anisotropy model is based on the basic postulate that an independent evolution of stress and structure is possible and the macroscopic modulus $A$ accounts for the deviatoric deformation history, being proportional to the microscopic rank-two deviatoric fabric $F_{\mathrm{dev}}$. More detailed explanations about the constitutive model and its parameters can be found in $[84,128,131]$.

The reduced model, with some simplifying assumptions as introduced in [84, 118, 121], reduces to only two independent evolution equations for the deviatoric stress ratio $s_{\mathrm{dev}}$, and the deviatoric fabric $F_{\mathrm{dev}}$, where the former is given by:

$$
s_{\mathrm{dev}}=s_{\mathrm{dev}}^{\max }-\left(s_{\mathrm{dev}}^{\max }-s_{\mathrm{dev}}^{0}\right) e^{-\beta_{s} \varepsilon_{\mathrm{dev}}}
$$

where $s_{\mathrm{dev}}^{0}$ and $s_{\mathrm{dev}}^{\max }$ represent the initial and maximum values of $s_{\mathrm{dev}}$ and $\beta_{s}$ is its growth 
rate. Similarly, the deviatoric fabric is approximated by:

$$
F_{\mathrm{dev}}=F_{\mathrm{dev}}^{\max }-\left(F_{\mathrm{dev}}^{\max }-F_{\mathrm{dev}}^{0}\right) e^{-\beta_{F} \varepsilon_{\mathrm{dev}}},
$$

where $F_{\mathrm{dev}}^{0}$ and $F_{\mathrm{dev}}^{\max }$ represent the initial and maximum (saturation) values of the deviatoric fabric, and $\beta_{F}$ is its rate of change.

\subsubsection{Calibration for polydisperse samples}

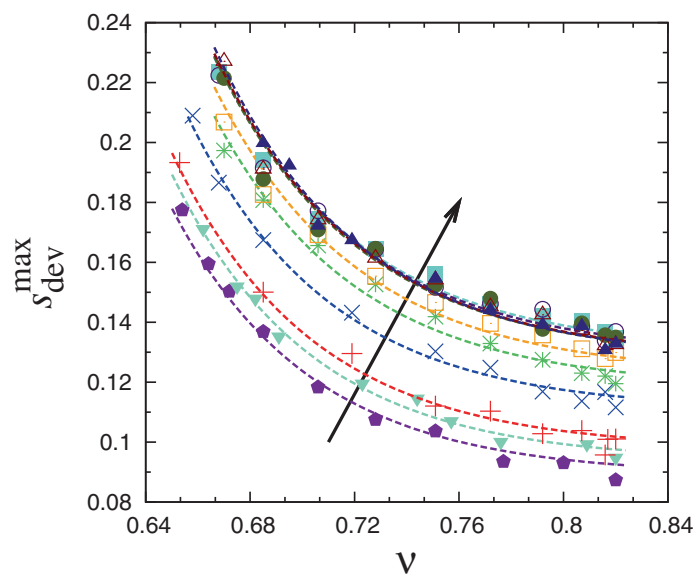

(a)

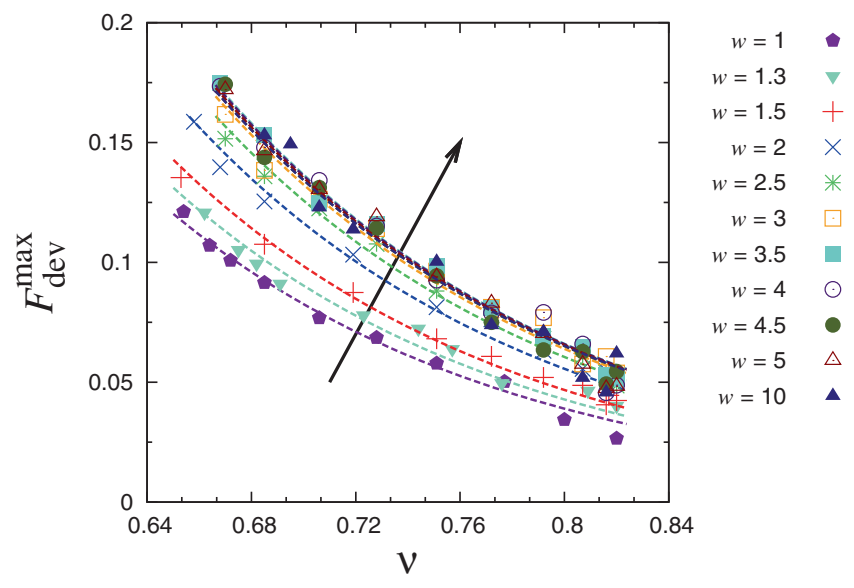

(b)

Figure 3.14: Comparison of evolution parameters for normalized deviatoric stress $s_{\mathrm{dev}}$ and $F_{\text {dev }}$ with polydispersity $w$ for the deviatoric deformation mode. (a) The maximum normalized deviatoric stress $s_{\mathrm{dev}}^{\max }$ plotted against volume fraction $v$. (b) The maximum deviatoric fabric $F_{\mathrm{dev}}^{\max }$ plotted against volume fraction $v$. The arrow indicates the increasing $w$. The corresponding dashed lines are the fit using Eq. (3.17).

In the following, we use these two equations as empirical fit functions, since they are special cases of the complete constitutive model with anisotropy, to deduce the model parameters as functions of volume fraction $v$ from various volume conserving deviatoric simulations [84]. In particular, the influence of polydispersity $w$ on the fitting parameters is studied. As an example, the deviatoric data for $w=1.5,2$ and 5 are fitted using Eqs. (3.15) and (3.16) and the four parameters $s_{\mathrm{dev}}^{\max }, \beta_{s}, F_{\mathrm{dev}}^{\max }$ and $\beta_{F}$ are extracted. The procedure is applied to the full set of polydisperse packings with many different $v$ (not shown).

Figs. 3.14a and 3.14b show the variation of $s_{\mathrm{dev}}^{\max }$ and $F_{\mathrm{dev}}^{\max }$ respectively with $v$, for different $w$. Both $s_{\mathrm{dev}}^{\max }$ and $F_{\mathrm{dev}}^{\max }$ decreases with increasing volume fraction $v$ and saturate towards a finite limit for large volume fractions. This is because for higher volume fractions, the motion of spheres is more constrained by more contacts and hence the anisotropy developed during the deformation is smaller. Also with increasing polydispersity, the steady state values of $s_{\mathrm{dev}}^{\max }$ and $F_{\mathrm{dev}}^{\mathrm{max}}$ increase, as explained in detail in section 3.4.3. 


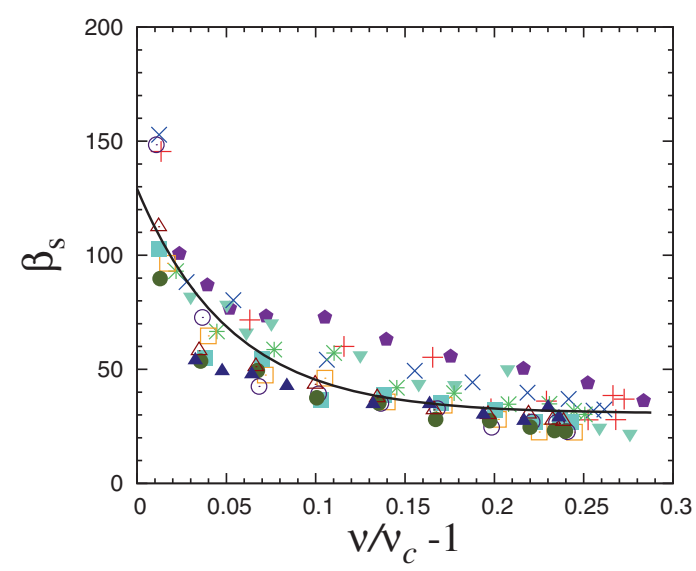

(a)

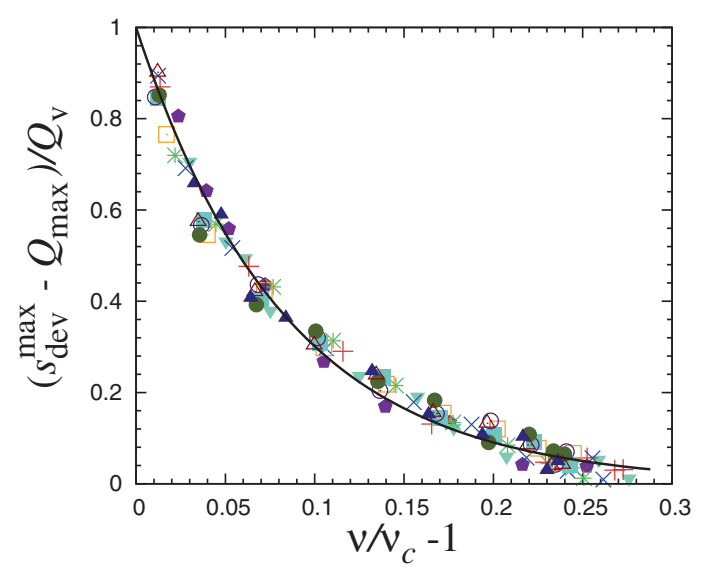

(c)

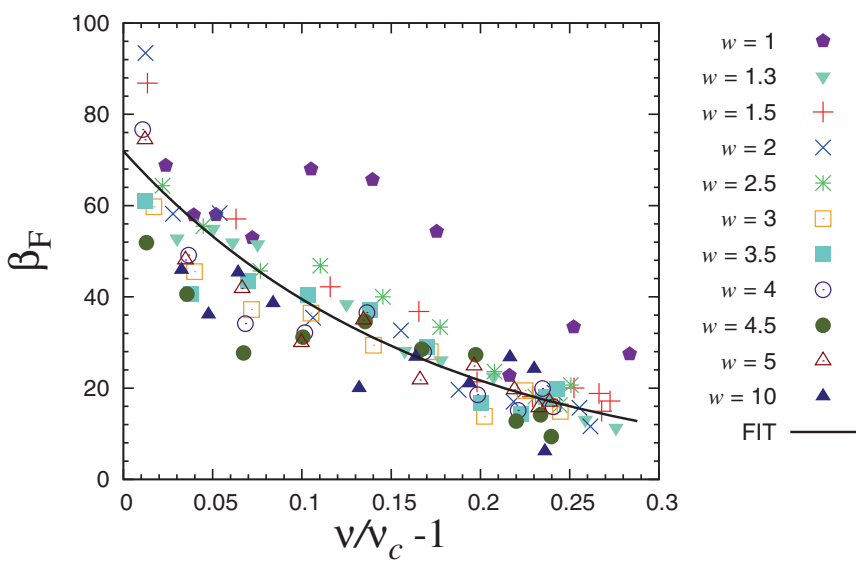

(b)

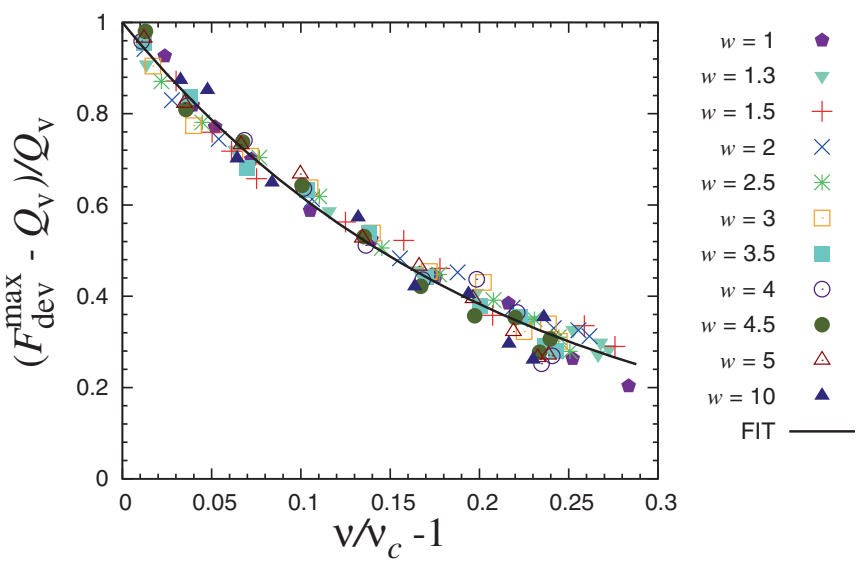

(d)

Figure 3.15: The growth rates (a) $\beta_{s}$ of $s_{\mathrm{dev}}$ and (b) $\beta_{F}$ of $F_{\mathrm{dev}}$ plotted against scaled volume fraction, $\left(v / v_{c}-1\right)$. Scaled (c) $s_{\mathrm{dev}}^{\max }$ and (d) $F_{\mathrm{dev}}^{\max }$ with components $Q_{\max }$ and $Q_{\mathrm{v}}$ (see Table 3.3) plotted against scaled volume fraction, $\left(v / v_{c}-1\right)$. The corresponding solid lines are the scaled parameters using Eq. (3.17) with data taken from Table 3.3.

Figs. $3.15 \mathrm{a}$ and $3.15 \mathrm{~b}$ show the variation of $\beta_{s}$ and $\beta_{F}$ respectively with $v$ relative to the jamming volume fraction, i.e. $v / v_{c}-1$, for different $w$ from the same deviatoric simulations as above. A decreasing trend is seen for $\beta_{s}$ versus $v / v_{c}-1$, with larger scatter when compared with $s_{\mathrm{dev}}^{\max }$. With increasing polydispersity, the trend in the growth rate $\beta_{s}$ with polydispersity $w$ is minimal (as shown in the inset of Fig. 3.11a), so we neglect this variation in this work. A similar decreasing trend in $\beta_{F}$ with $v / \nu_{c}-1$ is seen, while besides fluctuations, $\beta_{F}$ is weakly dependent on $w$. In Figs. 3.14 and 3.15, we also report the values of the four parameters for the monodisperse packing, $w=1$. We note that when $\beta_{s}$ and $\beta_{F}$ are plotted in Figs. 3.15a and $3.15 \mathrm{~b}$, the data for $w=1$ show anomalously large values. This is probably due to partial, local crystallization [175] present in the monodisperse case. 


\begin{tabular}{|c|c|c|c|c|c|c|c|c|c|c|c|}
\hline & & \multicolumn{3}{|c|}{$s_{\mathrm{dev}}^{\max }$} & \multicolumn{2}{|l|}{$F_{\mathrm{dev}}^{\max }$} & \multicolumn{3}{|c|}{$\beta_{s}$} & \multicolumn{2}{|l|}{$\beta_{F}$} \\
\hline$w$ & $v_{c}$ & $Q_{\max }$ & $Q_{\mathrm{v}}$ & $\alpha$ & $Q_{\mathrm{v}}$ & $\alpha$ & $Q_{\max }$ & $Q_{\mathrm{v}}$ & $\alpha$ & $Q_{\mathrm{v}}$ & $\alpha$ \\
\hline 1.0 & 0.6389 & 0.0888 & & & 0.1308 & & - & - & - & - & - \\
\hline 1.3 & 0.6427 & 0.0935 & \multirow{10}{*}{0.11} & \multirow{10}{*}{12} & 0.1386 & \multirow{10}{*}{4.8} & \multirow{10}{*}{31} & \multirow{10}{*}{116} & \multirow{10}{*}{22} & \multirow{10}{*}{72} & \multirow{10}{*}{6} \\
\hline 1.5 & 0.6444 & 0.0976 & & & 0.1491 & & & & & & \\
\hline 2.0 & 0.6500 & 0.1106 & & & 0.1684 & & & & & & \\
\hline 2.5 & 0.6557 & 0.1164 & & & 0.1741 & & & & & & \\
\hline 3.0 & 0.6587 & 0.1226 & & & 0.1789 & & & & & & \\
\hline 3.5 & 0.6599 & 0.1303 & & & 0.1830 & & & & & & \\
\hline 4.0 & 0.6609 & 0.1292 & & & 0.1810 & & & & & & \\
\hline 4.5 & 0.6614 & 0.1278 & & & 0.1777 & & & & & & \\
\hline 5.0 & 0.6620 & 0.1279 & & & 0.1782 & & & & & & \\
\hline 10.0 & 0.6634 & 0.1273 & & & 0.1751 & & & & & & \\
\hline
\end{tabular}

Table 3.3: Fitting coefficients for the parameters in Eqs. (3.15) and (3.16),using Eq. (3.17)) with $v_{c}(w)$, extracted from Table 3.4 using the deviatoric deformation mode, for various $w$.

A clear difference between the fit parameters of deviatoric stress and deviatoric fabric, namely the steady values $s_{\mathrm{dev}}^{\max }, F_{\mathrm{dev}}^{\max }$ (Figs. 3.14a and 3.14b), and the growth rates $\beta_{s}$ and $\beta_{F}$ (Figs. 3.15a and 3.15b) can be seen. This confirms that stress and fabric indeed evolve independently with deviatoric strain [84], as is the basic postulate for the anisotropy constitutive model.

We propose a generalized analytical relation to fit the stress parameters $s_{\mathrm{dev}}^{\max }, \beta_{s}$ and the fabric parameters $F_{\mathrm{dev}}^{\max }, \beta_{F}$, obtained from various different volume conserving deviatoric simulations. Their dependence on volume fraction $v$ (see. [84], for $w=3$ ), is well described by the general relation:

$$
Q=Q_{\max }(w)+Q_{\mathrm{v}}(w) \exp \left(-\alpha(w)\left(\frac{v}{v_{c}(w)}-1\right)\right),
$$

where $Q_{\max }(w), Q_{\mathrm{v}}(w)$ and $\alpha(w)$ are the fitting parameters with values presented in Table 3.3, $v$ is the volume fraction and $v_{c}(w)$ is the jamming volume fraction for the deviatoric deformation mode dependent on polydispersity $w$ (see Fig. 3.7). For all four parameters, $Q_{\max }(w)$ is the limit value for large volume fraction, $Q_{c}=Q_{\max }(w)+Q_{\mathrm{v}}(w)$ represents the limit at $v \rightarrow v_{c}(w)$, and $\alpha(w)$ is the rate of variation (decay) with the volume fraction.

Here we study and discuss the four cases separately. (1) For $s_{\text {dev }}^{\max }$, from Fig. 3.14a the variation of $Q_{\max }$ with $w$ is systematic and the curves are parallel. Hence $Q_{\mathrm{v}}$ and $\alpha$ can be 
considered independent of $w$. When the curves in $s_{\mathrm{dev}}^{\max }$ are scaled with the respective $Q_{\max }$ by $\left(s_{\mathrm{dev}}^{\max }-Q_{\max }(w)\right) / Q_{\mathrm{v}}$, this leads to a collapse, as shown in Fig. 3.15c. (2) For $\beta_{s}$, in this work, we neglect its weak variation with $w$ and assume constant values for the fit parameters $Q_{\max }, Q_{\mathrm{v}}$ and $\alpha$. When looking at the structural anisotropy, we assume, as consistent with the data, that both (3) $F_{\operatorname{dev}}^{\max }$ and (4) $\beta_{F}$ tend to 0 as the volume fraction increases, therefore we set $Q_{\max }=0$ in their fitting functions. We observe in Fig. 3.14b, that the variation of $Q_{\mathrm{v}}$ with $w$ is systematic for (3) $F_{\mathrm{dev}}^{\max }$. When $F_{\mathrm{dev}}^{\max }$ is scaled with $Q_{\mathrm{v}}$ by $F_{\mathrm{dev}}^{\max } / Q_{\mathrm{v}}(w)$, the data collapse as shown in Fig. 3.15d. Since the curves have the same trend $\alpha$ is set constant independent of $w$. As reported in the inset of Fig. 3.11b, (4) $\beta_{F}$ is independent of the initial configuration, that is $w$, and we set constant $Q_{\mathrm{v}}$ and $\alpha$ in this case. Interestingly, we can reduce Eq. (3.17) in a very compact form by expressing the two $w$-dependent parameters $Q_{\max }(w)$ for (1) $s_{\mathrm{dev}}^{\max }$, and $Q_{\mathrm{v}}(w)$ for (3) $F_{\mathrm{dev}}^{\max }$ as functions only of $v_{c}=v_{c}(w)$ :

$$
\begin{array}{rlrl}
s_{\operatorname{dev}}^{\max }(v, w) & =Q_{\max }\left(v_{c}\right)+Q_{\mathrm{v}} \exp \left(-\alpha\left(v / v_{c}-1\right)\right), \\
\beta_{s}(v, w) & =Q_{\max }+Q_{\mathrm{v}} \exp \left(-\alpha\left(v / v_{c}-1\right)\right), \\
F_{\operatorname{dev}}^{\max }(v, w) & = & Q_{\mathrm{v}}\left(v_{c}\right) \exp \left(-\alpha\left(v / v_{c}-1\right)\right), \\
\beta_{F}(v, w) & = & Q_{\mathrm{v}} \exp \left(-\alpha\left(v / v_{c}-1\right)\right),
\end{array}
$$

with $Q_{\max }=-1+1.7 v_{c}$ for (1) $s_{\mathrm{dev}}^{\max }$ and $Q_{\mathrm{v}}=-0.9+1.6 v_{c}$ for (3) $F_{\mathrm{dev}}^{\max }$. Using these two equations, everything in Eq. (3.18) can be expressed as either constant, or as function of $v_{c}$, that become a unique state variable able to describe the history of the material due to its deformation mode. Using these equations, together with $v_{c}$ data from Table 3.2, and constant parameters from Table 3.3, we can describe the variation in the parameters $s_{\mathrm{dev}}^{\max }, \beta_{s}, F_{\mathrm{dev}}^{\max }$ and $\beta_{F}$ with volume fraction $v$ and polydispersity $w$, and use them to predict the behavior during uniaxial deformation.

\subsubsection{Prediction of uniaxial deformation for polydisperse samples}

Figure $3.12 \mathrm{a}$ shows the deviatoric stress ratio $s_{\mathrm{dev}}$ plotted against deviatoric strain $\varepsilon_{\mathrm{dev}}$ for uniaxial deformations, compared with the predictions of Eq. (3.15) with coefficients $s_{\mathrm{dev}}^{\max }(v, w)$ and $\beta_{s}(v, w)$ taken from Eqs. (3.18a) and (3.18b). The proposed model, although in its simplified version, is able to properly capture the behavior of the material qualitatively, $s_{\text {dev }}$ approaching exponentially a maximum value and then decreasing due to the volume fraction and polydispersity dependence of the parameters.

Figure $3.12 \mathrm{~b}$ shows the evolution of deviatoric fabric, $F_{\mathrm{dev}}$, with deviatoric strain, $\varepsilon_{\mathrm{dev}}$, for uniaxial deformations - as above - together with the predictions of Eq. (3.16), with parameters taken from Eqs. (3.18c) and (3.18d). The model is still able to qualitatively describe the behavior of the deviatoric fabric, but with order of $30 \%$ over-prediction for large strain. Note that the softening present in some of the deviatoric DEM data, is on purpose not plugged into the model as a constraint, which renders the weak softening present in some of the uniaxial 
data as a valuable prediction of the model. For better understanding, the complete coupled model needs to be used and possibly improved, as will be presented elsewhere.

\subsection{Summary and Outlook}

We use the discrete element method to investigate the behavior of three-dimensional frictionless granular assemblies characterized by different polydispersities and subjected to various deformation paths. In particular isotropic loading/unloading, deviatoric (pure) shear, and uniaxial compression are studied.

The main goal is to analyze and understand the reciprocal influence of polydispersity and deformation history on the response of the material, where the structural/bulk effects are highlighted by using the simplest linear visco-elastic contact model. The evolution of the scaled pressure as a function of volumetric strain (relative to the jamming volume fraction $v_{c}$ ) is well described by an analytical (linear, to very good approximation) scaling equation (3.10). This shows that the isotropic fabric is proportional to the isotropic stress - when proper parameters depending slightly on the deformation mode are included. Notably, only the jamming volume fraction, among the fit parameters for the pressure, describes the role of both polydispersity and deformation history on the material behavior. As reported earlier in [84], the isotropic jamming volume fraction $v_{c}$ is not a single value for a particular system configuration but it is strongly dependent on the deformation mode and history of the packing. Moreover, $v_{c}$ increases with polydispersity, following the behavior described in [147], with the isotropic and deviatoric tests giving the highest and lowest values, respectively, while the uniaxial dataset lies in between. On the contrary, the shear jamming volume fraction, slightly below the isotropic jamming volume fraction, has been confirmed as a lower limit value in recent studies, independent of the deformation path [21]. The detailed simulations by [147], using hard instead of soft spheres, represent lower or upper bounds to $v_{c}$ if they are carried out extremely fast or slow, respectively. However, the relation between these distinct results has to be further studied elsewhere.

When the micromechanics is analyzed, the coordination number decreases with polydispersity, while the fraction of rattlers displays an opposite trend, increasing with $w$. In these cases, the evolution of the state variables can be predicted by using the evolution equations from [69], with parameters dependent on the polydispersity of the packing, while the laws for the critical volume fraction $v_{c}(w)$ as extrapolated from the pressure behavior are used. Interestingly, the free fit parameters are not affected by the deformation modes in the case of the micromechanical quantities, that is they are fully described by the evolution of the critical volume fraction, acting as history variable for the sample.

The behavior of polydisperse systems are predicted by [147], to depend on the moments of their size distribution, after the rattlers are excluded. Since for larger $w$, the moments 
(scaled by $\langle r\rangle$ ) do not change much above $w \approx 3-4$, which explains the saturation of many quantitities - for the uniform size distribution used in this work.

During deviatoric and uniaxial deformations, both deviatoric stress ratio and deviatoric fabric evolve with the deviatoric strain, reaching saturation values that increase with polydispersity. The initial growth rate of stress, $\beta_{s}$, weakly depends on polydispersity, due to the relation between the shear stiffness of isotropic samples and the volumetric fabric $F_{\mathrm{v}}(w)$. On the other hand the growth rate of deviatoric fabric $\beta_{F}$ is fairly independent of polydispersity (besides fluctuations), showing that the incremental response of the granular deviatoric fabric is not directly related to its isotropic state $F_{\mathrm{v}}$.

The DEM data of the volume conserving deviatoric tests are used to calibrate a simple constitutive model that involves anisotropy as proposed in 2D by [128, 131]. The four parameters that characterize the model $s_{\mathrm{dev}}^{\max }, \beta_{s} F_{\mathrm{dev}}^{\max }$ and $\beta_{F}$ are expressed as functions of volume fraction and polydispersity. They show a very similar behavior decreasing with an exponential law from a maximum value at the jamming volume fraction to a saturation minimum. Also in this case, where only two parameters are depending on $w$ and thus $v_{c}(w)$, the dependence on polydispersity can be fully described through the established variation of the jamming volume fraction $v_{c}(w)$ with $w$.

As final step, the constitutive model calibrated on deviatoric data is used to predict both stress and fabric evolution under uniaxial deformation - with very good qualitative success and within $70-80 \%$ quantitative agreement. The prediction of the uniaxial test shows promising perspectives for future research. The basic qualitative features are captured by the model, even though it is used in a very idealized and short form, with the single anisotropy modulus. In the future, the coupled equations have to be solved and additional formulations/terms that relate anisotropy (possibly a second anisotropy modulus) with the deviatoric fabric will also be investigated. Moreover, it would be interesting to look deeper into different distributions of polydispersity like constant volume fraction, or log-normal distributions. 


\section{A Table of parameters}

\begin{tabular}{|c|c|c|c|c|c|c|}
\hline$w$ & $v_{c}$ & $p_{0}$ & $\gamma_{p}$ & $C_{1}$ & $\phi_{c}$ & $\phi_{\mathrm{v}}$ \\
\hline \multicolumn{7}{|c|}{ ISO } \\
\hline 1.0 & 0.6478 & 0.0430 & 0.2131 & 9.0622 & 0.0171 & 46.7722 \\
\hline 1.3 & 0.6491 & 0.0432 & 0.2010 & 9.0053 & 0.0220 & 40.5552 \\
\hline 1.5 & 0.6514 & 0.0430 & 0.1698 & 8.9759 & 0.0309 & 35.2452 \\
\hline 2.0 & 0.6577 & 0.0428 & 0.1299 & 8.8795 & 0.0650 & 28.6337 \\
\hline 2.5 & 0.6624 & 0.0421 & 0.0499 & 8.7233 & 0.1010 & 20.2312 \\
\hline 3.0 & 0.6648 & 0.0419 & 0.0720 & 8.5585 & 0.1559 & 17.6338 \\
\hline 3.5 & 0.6668 & 0.0419 & 0.1481 & 8.4082 & 0.1818 & 13.4036 \\
\hline 4.0 & 0.6674 & 0.0425 & 0.1882 & 8.2977 & 0.2049 & 10.5633 \\
\hline 4.5 & 0.6675 & 0.0424 & 0.2409 & 8.1672 & 0.2417 & 9.8332 \\
\hline 5.0 & 0.6680 & 0.0428 & 0.2825 & 8.1636 & 0.2513 & 8.0380 \\
\hline 10.0 & 0.6696 & 0.0444 & 0.3992 & 8.1674 & 0.3210 & 4.6514 \\
\hline \multicolumn{7}{|c|}{ UNI } \\
\hline 1.0 & 0.6423 & 0.0398 & 0.0776 & 8.7464 & 0.0212 & 39.8092 \\
\hline 1.3 & 0.6440 & 0.0399 & 0.0808 & 8.6618 & 0.0254 & 35.2456 \\
\hline 1.5 & 0.6463 & 0.0393 & -0.0025 & 8.6241 & 0.0309 & 32.7265 \\
\hline 2.0 & 0.6525 & 0.0387 & -0.0840 & 8.5253 & 0.0734 & 26.0018 \\
\hline 2.5 & 0.6576 & 0.0383 & -0.1974 & 8.3847 & 0.1148 & 20.3461 \\
\hline 3.0 & 0.6605 & 0.0376 & -0.1962 & 8.2066 & 0.1640 & 16.0260 \\
\hline 3.5 & 0.6625 & 0.0384 & -0.0793 & 8.1357 & 0.2018 & 13.2581 \\
\hline 4.0 & 0.6634 & 0.0388 & 0.0086 & 7.9881 & 0.2359 & 10.8769 \\
\hline 4.5 & 0.6644 & 0.0390 & 0.0081 & 7.9333 & 0.2531 & 9.2102 \\
\hline 5.0 & 0.6647 & 0.0386 & -0.0527 & 7.8750 & 0.2622 & 7.9085 \\
\hline 10.0 & 0.6662 & 0.0416 & 0.2482 & 7.9177 & 0.3342 & 4.4610 \\
\hline
\end{tabular}

Table 3.4: Summary of parameters used in Eqs. (3.10), (3.12) with $C_{0}=6, \alpha=0.60$ for the three modes, and (3.13) with polydispersity $w$. 


\begin{tabular}{|c|c|c|c|c|c|c|}
\hline$w$ & $v_{c}$ & $p_{0}$ & $\gamma_{p}$ & $C_{1}$ & $\phi_{c}$ & $\phi_{\mathrm{v}}$ \\
\hline \multicolumn{7}{|c|}{ DEV } \\
\hline 1.0 & 0.6389 & 0.0363 & -0.0954 & 8.6689 & 0.0281 & 46.0916 \\
\hline 1.3 & 0.6427 & 0.0405 & 0.1771 & 8.6137 & 0.0249 & 42.2059 \\
\hline 1.5 & 0.6444 & 0.0399 & 0.1223 & 8.5451 & 0.0476 & 39.7536 \\
\hline 2.0 & 0.6500 & 0.0387 & -0.0215 & 8.4097 & 0.0744 & 27.1618 \\
\hline 2.5 & 0.6557 & 0.0396 & 0.0594 & 8.3101 & 0.1028 & 19.4110 \\
\hline 3.0 & 0.6587 & 0.0396 & 0.0924 & 8.1634 & 0.1453 & 15.2955 \\
\hline 3.5 & 0.6599 & 0.0386 & 0.0382 & 7.9801 & 0.1881 & 12.3952 \\
\hline 4.0 & 0.6609 & 0.0388 & 0.0744 & 7.8672 & 0.2131 & 9.8732 \\
\hline 4.5 & 0.6614 & 0.0393 & 0.1539 & 7.7965 & 0.2336 & 8.5445 \\
\hline 5.0 & 0.6620 & 0.0396 & 0.1793 & 7.4895 & 0.2492 & 7.3233 \\
\hline 10.0 & 0.6634 & 0.0419 & 0.3617 & 7.7373 & 0.3114 & 3.8805 \\
\hline
\end{tabular}

Table 3.4 continued summarizing the parameters for the deviatoric deformation mode. 


\title{
CHAPTER 4
}

\section{Constitutive model with anisotropy for granular materials*}

\begin{abstract}
Physical experiments characterize the elastic response of granular materials in terms of macroscopic variables, namely volume fraction and stress, while the microstructure is not known and thus neglected. Here, by means of numerical simulations, we analyze dense, frictionless, granular assemblies with the final goal to relate the elastic moduli to the fabric state, i.e., the micro-structural averaged contact network.

The particle samples are first isotropically compressed and later quasi-statically sheared under constant volume (undrained conditions). From various static configurations at different shear strains, now infinitesimal strain step are applied to "measure" the effective elastic response, while plasticity in the sample develops as soon as contact rearrangements happen. Because of the anisotropy induced by shear, volumetric and deviatoric stresses and strains are cross-coupled via two anisotropy moduli. Besides the expected dependence of the bulk modulus on the isotropic fabric, we find that the ratio of anisotropy moduli and bulk modulus scales with the deviatoric fabric. Interestingly, the shear modulus of the material depends also on the actual stress state, along with the contact configuration.
\end{abstract}

Finally, a constitutive model based on incremental evolution equations for stress and

*. Based on N. Kumar, S. Luding, and V. Magnanimo. Macroscopic model with anisotropy based on micromacro informations. Acta Mechanica, Accepted, 2014 
fabric is introduced. By knowing the dependence of the stiffness tensor (elastic moduli) on the microstructure, the theory, as calibrated during the shear deformation, is able to predict with good agreement the evolution of pressure, shear stress and deviatoric fabric for an independent undrained cyclic shear test.

\subsection{Introduction}

Granular materials behave differently from usual solids or fluids and show peculiar mechanical properties like dilatancy, history dependence, ratcheting and anisotropy $[69,70,73,84$, $103,104,145,178,190,193,222]$. The behavior of these materials is highly non-linear and involves plasticity even at very small strain due to rearrangements of the elementary particles $[20,45,65]$. The concept of an initial purely elastic regime (small strain) for granular assemblies is an issue still under debate in the mechanical and geotechnical communities. On the other hand, approaches that neglect the effect of elastic stored energy, i.e., where all the work done by the internal forces is dissipated, are also questionable. Features visible in experiments, like wave propagation, can hardly be described without elastic response. In a general picture, both the deformations at contacts and the irrecoverable rearrangements of the grains sum up to the total strain. The former represents the elastic, reversible contribution to the behavior of the material. That is, for very small strain the response of a finite granular system in static equilibrium can be assumed to be linearly elastic [57, 107, 144, 178], as long as no irreversible rearrangements take place.

Despite these arguments and the long-standing debate, basic features of the physics of granular elasticity are currently unresolved, such as the determination of a proper set of state variables to describe the average moduli. Physical experiments carried out on sand and glass beads show that wave propagation in the aggregate depends upon the stress state and the volume fraction [19, 56, 87, 94, 107, 211, 215]. Recent works [3, 73, 94, 110, 222] show that along with the macroscopic properties (stress and volume fraction) [56, 94, 219], the structure, quantified by the fabric tensor [36, 120, 146, 178, 222] plays also a crucial role, as it characterizes, on average, the geometric arrangement of contacts. In particular, when the material is sheared, anisotropy in the contact network develops, due to the opening and closing of contacts, restructuring, and the creation and destruction of force-chains. The anisotropic state is at the origin of interesting observations on wave propagation in sheared granular media. The mechanical behavior of anisotropic soils is a topic of current interest for both experimental and theoretical investigations. As one example, extensive experimental studies of anisotropy have been carried out on laboratory-prepared (by careful 'raining' or bedding) sand specimens $[52,216]$. These and other studies show that the sample deformation characteristics depends highly on the orientation of the bedding plane with respect to the principal stress and fabric axes.

Most standard constitutive models, involving elasticity and/or plasticity have been applied 
to describe the incremental behavior of (an)isotropic granular solids - sometimes with success, but typically only in a limited range of parameters. In the majority of the models, the stiffness is related to the actual stress state of the granular system and its density. This is the case for hypoplasticity [68, 94], where a single non-linear tensorial equation relates the Jaumann stress-rate with strain-rate and stress tensors. Only a few theories after the pioneering work by Cowin [40], see e.g. [29, 30, 43, 142, 143, 190, 193] and references therein, consider explicitly the influence of the micro-mechanic structure on the elastic stiffness, plastic flow-rule or noncoaxiality of stress and strain. The evolution of microstructure due to deformation is an essential part of a constitutive model for granular matter because it contains the information how different paths have affected the mechanical state of the system. In this sense, fabric is a tensorial history variable. When included in the formulation, the effect of structure is often described by a fixed fabric tensor normal to the bedding plane of deposited sands [43, 114, 193, 213]. Recently Li \& Dafalias [115] have proposed a new framework (rather than a specific constitutive model) by reconsidering the classical steady state theory by Roscoe et al. [169], with a fabric tensor evolving towards a properly defined steady state value. This is supported by experimental [216] and extensive numerical works $[73,84,120,195,222]$. In a similar fashion, the anisotropy model proposed in $[128,131]$ postulates the split of isotropic and deviatoric stress, strain and fabric and includes the microstructure as a variable, whose behavior is described by an evolution equation independent of stress. Refs. [84, 103] predicts uniaxial simulation results under this assumption (independent evolution of stress and structure), where the simplified model well captures the qualitative behavior.

In this work we use the Discrete Element Method (DEM) to study granular assemblies made of polydisperse frictionless particles and focus on their elastic behavior. By isolating elasticity we aim to distinguish the kinematics at the microscale that lead to either macroscopic elasticity or plasticity. We analyze the role of microstructure, stress state and volume fraction on the evolution of the elastic moduli, with the goal of characterizing all of them in terms of a unique, limited set of variables. In order to calculate the stiffness tensor, we apply small-strain probes to various equilibrium states along a volume conserving (undrained) shear deformation path. In the case of a finite assembly of particles, in simulations, a finite elastic regime can always be detected and the elastic stiffnesses can be measured by means of an actual, very small, strain perturbation [130]. The purpose is to improve the understanding of elasticity in particle systems and to guide further developments for new constitutive models. As an example, the relation between moduli and fabric here is used in the anisotropic constitutive model, as proposed in $[128,131]$, to predict the macroscopic behavior during a more general deformation path, involving also strain reversal.

This chapter is organized as follows: The simulation method and parameters used and the averaging definitions for scalar and tensorial quantities are given in section 4.2. The preparation test procedures, and the results from the deviatoric simulation are explained in section 4.3. Section 4.4 is devoted to the measurement of elastic moduli by means of small isotropic 
and deviatoric perturbations. There we present the evolution of the moduli with strain and link them to the state variables. Finally, section 4.5 is devoted to theory, where we relate the evolution of the microstructural anisotropy to that of stress and strain, as proposed in Refs. $[128,131]$. This displays the predictive quality of the model, calibrated only for isochoric, uni-directional shear, when applied to an independent, cyclic shear test.

\subsection{Numerical simulation}

The Discrete Element Method (DEM) [42] has been used extensively in performing simulations in biaxial and triaxial geometries [50, 99, 121, 190] involving advanced contact models for fine powders [122], or general deformation modes, see [5, 195, 197] and references therein. In this work, however, we restrict ourselves to the simplest deformation tests - namely isotropic, uniaxial and deviatoric modes - and to the linear contact model without friction. A detailed description about the contact model used in the DEM simulations can be found in section 2.2 of Chapter 2.

The standard simulation parameters are, $N=9261\left(=21^{3}\right)$ particles with average radius $\langle r\rangle=1[\mathrm{~mm}]$, density $\rho=2000\left[\mathrm{~kg} / \mathrm{m}^{3}\right]$, elastic stiffness $k=10^{8}\left[\mathrm{~kg} / \mathrm{s}^{2}\right]$, particle damping coefficient $\gamma=1[\mathrm{~kg} / \mathrm{s}]$, background dissipation $\gamma_{b}=0.1[\mathrm{~kg} / \mathrm{s}]$. Note that the polydispersity of the system is quantified by the width $\left(w=r_{\max } / r_{\min }=3\right)$ of a uniform size distribution [69], where $r_{\max }$ and $r_{\min }$ are the radii of the biggest and smallest particles respectively.

The average time scale when two averaged size particles (with $r_{\mathrm{avg}}=\langle r\rangle=1$ ) with mass $m_{\mathrm{avg}}=\rho\left(4 \pi r_{\mathrm{avg}}^{3} / 3\right)=8.377[\mu \mathrm{g}]$ interact is given as $t_{c, \mathrm{avg}}=\pi / \sqrt{k / m_{\mathrm{avg}}^{\prime}-\left(\gamma /\left(2 m_{\mathrm{avg}}^{\prime}\right)\right)^{2}}$ $=0.6431[\mu \mathrm{s}]$, where $m_{\mathrm{avg}}^{\prime}=m_{\mathrm{avg}} / 2$ is the reduced mass, with restitution coefficient $e_{\mathrm{avg}}=$ $\exp \left(-\gamma t_{c, \text { avg }} /\left(2 m_{\text {avg }}^{\prime}\right)\right)=0.926$. The fastest response time scale in the system is determined when two smallest particle with mass $m_{\text {small }}=\rho\left(4 \pi r_{\min }^{3} / 3\right)=1.047[\mu \mathrm{g}]$ interact, and is given as $t_{c, \text { small }}=\pi / \sqrt{k / m_{\text {small }}^{\prime}-\left(\gamma /\left(2 m_{\text {small }}^{\prime}\right)\right)^{2}}=0.2279[\mu \mathrm{s}]$, where $m_{\text {small }}^{\prime}=m_{\text {small }} / 2$ is the reduced mass, with restitution coefficient $e_{\text {small }}=\exp \left(-\gamma t_{c \text {,small }} /\left(2 m_{\text {small }}^{\prime}\right)\right)=0.804$.

\subsubsection{Coordination number and fraction of rattlers}

The classical definition of coordination number is $C=M / N$, where $M$ is the total number of contacts and $N=9261$ is the total number of particles. The corrected coordination number is $C^{*}=M_{4} / N_{4}$, where, $M_{4}$ is the total number of contacts of the $N_{4}$ particles with at least 4 contacts $[69,84,103]$. . Moreover, we introduce here the reduced number of contacts $M_{4}^{p}$, where contacts related to rattlers are excluded twice, as they do not contribute to the stability of both the rattler and the particle in contact with it. Hence, $M_{4}^{p}=M_{4}-M_{1}-M_{2}-M_{3}=$ $M-2\left(M_{1}+M_{2}+M_{3}\right)$, where $M_{1}, M_{2}$ and $M_{3}$ are total number of contacts of particles with only 1, 2 and 3 contacts respectively. This leads to a modification in corrected coordination 
number is $C_{p}^{*}=M_{4}^{p} / N_{4}$. The fraction of rattlers is $\phi_{r}=\left(N-N_{4}\right) / N$, hence, $C=C^{*}\left(1-\phi_{r}\right)$. The total volume of particles is $\sum_{\mathcal{P}=1}^{N} V_{\mathcal{P}}=4 \pi N\left\langle r^{3}\right\rangle$, where $\left\langle r^{3}\right\rangle / 3$ is the third moment of the size distribution $[69,103]$ and volume fraction is defined as $v=(1 / V) \sum_{\mathcal{P}=1}^{N} V_{\mathcal{P}}$, where $V$ is the volume of the periodic system.

\subsubsection{Macroscopic (tensorial) quantities}

Here, we focus on defining averaged tensorial macroscopic quantities - including strain-, stress- and fabric (structure) tensors - that provide information about the state of the packing and reveal interesting bulk features.

By speaking about the strain-rate tensor $\mathbf{E}$, we refer to the external strain that we apply to the sample. The isotropic part of the infinitesimal strain tensor $\varepsilon_{\mathrm{v}}[69,84,103]$ is defined as:

$$
\delta \varepsilon_{\mathrm{v}}=-\dot{\varepsilon}_{\mathrm{v}} \mathrm{dt}=-\frac{\delta \varepsilon_{x x}+\delta \varepsilon_{y y}+\delta \varepsilon_{z z}}{3}=-\frac{1}{3} \operatorname{tr}(\delta \mathbf{E})=-\frac{1}{3} \operatorname{tr}(\dot{\mathbf{E}}) \mathrm{dt},
$$

where $\varepsilon_{\alpha \alpha}=\dot{\varepsilon}_{\alpha \alpha} \mathrm{dt}$ with $\alpha \alpha=x x, y y$ and $z z$ as the diagonal components of the tensor in the Cartesian $x-y-z$ reference system. The trace integral of $3 \varepsilon_{\mathrm{v}}$ is denoted as the volumetric strain $\varepsilon_{v}$, the true or logarithmic strain, i.e., the volume change of the system, relative to the initial reference volume, $V_{0}$.

On the other hand, from DEM simulations, one can measure the 'static' stress in the system [35] as

$$
\boldsymbol{\sigma}=(1 / V) \sum_{c \in V} \mathbf{l}^{c} \otimes \mathbf{f}^{c}
$$

averaged over all the contacts in the volume $V$ of the dyadic products between the contact force $\mathbf{f}^{c}$ and the branch vector $\mathbf{l}^{c}$, where the contribution of the kinetic fluctuation energy has been neglected $[84,120]$. The isotropic component of the stress is the pressure $P=\operatorname{tr}(\boldsymbol{\sigma}) / 3$.

In order to characterize the geometry/structure of the static aggregate at microscopic level, we will measure the fabric tensor, defined as

$$
\mathbf{F}=\frac{1}{V} \sum_{\mathcal{P} \in V} V^{\mathcal{P}} \sum_{c \in \mathcal{P}} \mathbf{n}^{c} \otimes \mathbf{n}^{c}
$$

where $V^{\mathcal{P}}$ is the particle volume for particle $\mathcal{P}$, which lies inside the averaging volume $V$, and $\mathbf{n}^{c}$ is the normal unit branch-vector pointing from center of particle $\mathcal{P}$ to contact $c$ [104, 120, 219]. We want to highlight that a different, convention for the fabric tensor involves only the orientation of contacts as follows [146, 173, 222]:

$$
\mathbf{F}^{o}=\frac{1}{N_{c}} \sum_{c \in N_{c}} \mathbf{n}^{c} \otimes \mathbf{n}^{c}
$$


where $N_{c}$ is the total number of contacts in the system. An approximated relationship between Eqs. (4.3) and (4.4) can be derived as:

$$
\mathbf{F}^{o} \approx \frac{3 \mathbf{F}}{\operatorname{tr}(\mathbf{F})}
$$

with $\operatorname{tr}\left(\mathbf{F}^{o}\right)=1$. This relation is exactly equal for monodisperse assemblies but largely deviates for assemblies with high polydispersity (discussed in section 4.3). The difference also becomes more significant when the jamming volume fraction $[133,210]$ is approached, as the fraction of rattlers increases significantly, since Eq. (4.3) includes the volume of the particle as weighting factor in the definition (or the volume fraction of the sample). In the following, when not explicitly stated, we will refer to Eq. (4.3), since we combine the effects of volume fraction and number/orientation of contacts, both relevant quantities when the elastic moduli are considered [69].

In a large volume with a given distribution of particle radii, the relation between the isotropic fabric, i.e., the trace of $\mathbf{F}$ is proportional to the volume fraction $v$ and the coordination number $C$, as given in Refs. [69, 84, 103] as

$$
F_{\mathrm{v}}=\operatorname{tr}(\mathbf{F})=g_{3} v C=g_{3} v C^{*}\left(1-\phi_{r}\right),
$$

where $C, C^{*}$ and $\phi_{r}$ have been introduced in section 4.2 .1 and $g_{3} \approx 1.22$ for polydispersity $w=3$, being only a weighted, non-dimensional moments of the size distribution $[69,103$, 179].

\subsubsection{Isotropic and Deviatoric tensor parts}

We choose here to describe each symmetric second order tensor $\mathbf{Q}$, in terms of its isotropic part (first invariant) and the second $J_{2}$ and third $J_{3}$ invariants of the deviator:

$$
J_{2}=\frac{1}{2}\left[\left(Q_{1}^{D}\right)^{2}+\left(Q_{2}^{D}\right)^{2}+\left(Q_{3}^{D}\right)^{2}\right]
$$

and

$$
J_{3}=\operatorname{det}\left(\mathbf{Q}^{D}\right)=Q_{1}^{D} Q_{2}^{D} Q_{3}^{D},
$$

with $Q_{1}^{D}, Q_{2}^{D}$ and $Q_{3}^{D}$ eigenvalues of the deviatoric tensor $\mathbf{Q}^{D}=\mathbf{Q}-(\operatorname{tr}(\mathbf{Q}) / 3) \mathbf{I}$. We use the following definition to describe with a single scalar quantity the deviatoric part $[103,104]$ of Q:

$Q_{\mathrm{dev}}=\operatorname{Fsgn}(\mathbf{Q}) \sqrt{\frac{\left(Q_{x x}-Q_{y y}\right)^{2}+\left(Q_{y y}-Q_{z z}\right)^{2}+\left(Q_{z z}-Q_{z z}\right)^{2}+6\left(Q_{x y}^{2}+Q_{y z}^{2}+Q_{z x}^{2}\right)}{2}}$,

where $Q_{x x}, Q_{y y}$ and $Q_{z z}$ are its diagonal, and $Q_{x y}, Q_{y z}$ and $Q_{z x}$ its off-diagonal components. $\operatorname{Fsgn}(\mathbf{Q})$ is the sign function dependent on the deformation path. For the deviatoric deformations, as introduced in section 4.3 , we will use

$$
\operatorname{Fsgn}(\mathbf{Q})=\operatorname{sgn}\left(Q_{y y}-Q_{x x}\right)
$$


with $x$-wall expanding, $y$-wall compressing and $z$-wall non-mobile [103]. Eq. (4.7) relates the scalar representation to the second invariant of the deviatoric tensor through the relation $Q_{\mathrm{dev}}=\sqrt{3 J_{2}}$, where the deviators $\varepsilon_{\mathrm{dev}}, \sigma_{\mathrm{dev}}$ and $F_{\mathrm{dev}}$ refer to strain $\mathbf{E}$, stress $\boldsymbol{\sigma}$ and fabric $\mathbf{F}$, respectively. We want to point out here that, during a deformation, the response of stress $\boldsymbol{\sigma}$ and fabric $\mathbf{F}$ is opposite in sign to applied strain $\mathbf{E}$. Unless mentioned explicitly, we will be using a sign convention for strain (isotropic $\delta \varepsilon_{\mathrm{v}}=-1 / 3 \operatorname{tr}(\delta \mathbf{E})$ and deviatoric $\delta \varepsilon_{\mathrm{dev}}=$ $\left.-\delta E_{\mathrm{dev}}\right)$, such that consistently a positive strain leads to a positive stress and fabric response. Finally we note that in this work, we will use $k^{*}=k /(2\langle r\rangle)$ to non-dimensionalize pressure $P$ and deviatoric stress $\sigma_{\mathrm{dev}}$ to give $P^{*}$ and $\sigma_{\mathrm{dev}}^{*}$ respectively. Note that we will be referring deviatoric stress as shear stress in this study. ${ }^{1}$

\subsection{Volume conserving (undrained) biaxial shear test}

After the initial isotropic preparation, the packing is deformed following the deviatoric element test mode, a detailed procedure can be found in section 2.3.3 of Chapter 2. Starting from various $v_{i}$ chosen from the unloading branch $[84,103]$, the samples are then sheared keeping the total volume constant, that is with a strain-rate tensor

$$
\dot{\mathbf{E}}=\dot{\varepsilon}_{\mathrm{dev}}\left[\begin{array}{ccc}
-1 & 0 & 0 \\
0 & 1 & 0 \\
0 & 0 & 0
\end{array}\right]
$$

where $\dot{\varepsilon}_{\mathrm{dev}}=28.39\left[\mathrm{~s}^{-1}\right]$ is the strain-rate (compression $>0$ ) amplitude applied to the moving $x$ - and $y$-walls, while the third $z$-wall is stationary. Our shear test, where the total volume is conserved during deformation, resembles the undrained test typical in geotechnical practice [222]. The chosen deviatoric path is on the one hand similar to the pure-shear situation, and on the other hand allows for simulation of the biaxial element test $[141,166]$ (with two walls static, while four walls are moving, in contrast to the more difficult isotropic compression, where all the six walls are moving). Pure shear is here used to identify constant volume deviatoric loading with principal strain axis keeping the same orientation as the geometry (cuboidal) of the system for the whole experiment. In this case, there is no rotation (vorticity) of the principal strain (rate) axis and no distortion/rotation of the sample due to shear deformation. Different types of volume conserving deviatoric deformations can be applied to shear the system, but very similar behavior has been observed [84], in terms of shear stress.

1. It is important to point out that the rattlers are excluded in defining the (corrected) coordination number $C^{*}$. However dynamic rattler particles with $1 \leq M_{p} \leq 3$ contacts are included in the definitions of fabric and stress. We verified that during shear deformation, the maximum contribution in deviatoric stress due to rattlers is $0.03 \%$, while in the case of deviatoric fabric the contribution rises to $0.5 \%$. This is not surprising since only contacting particles contribute to the definitions of both stress and fabric and dynamic rattlers have a smaller weight for stress than for fabric, see Eq. (4.3). Note also that the number of rattlers decreases with increasing size of the particles [103]. 


\subsubsection{Evolution of stress}

The evolution of non-dimensional pressure $P^{*}$ with deviatoric strain $\varepsilon_{\mathrm{dev}}$ is presented in Fig. 4.1a during undrained shear tests for some exemplary volume fraction. For frictionless systems analyzed here, only a slight variation of the pressure is observed at the beginning of the test, due to the development of anisotropy in the sample, after which $P^{*}$ remains constant. ${ }^{3}$ Both the (small) initial pressure change and the final saturation value vary with the vicinity of $v$ to the jamming volume fraction $v_{c}$. Interestingly, depending on the volume fraction, some of the samples show increase of the pressure (dilatancy) with respect to the initial value and some other decrease (compactancy), as shown in Fig. 4.1. This supports the idea of a certain threshold value $v_{d}^{p}=0.79$, as shown in Fig. $4.2 \mathrm{a}$, for the behavior of the system similarly to the switch between volumetric dilation and contraction visible in triaxial tests.

The evolution of the (non-dimensional) shear stress $\sigma_{\mathrm{dev}}^{*}$ during shear, as function of the deviatoric strain $\varepsilon_{\mathrm{dev}}$, is shown in Fig. 4.1b, for the same simulations as in Fig. 4.1a. The stress grows with applied strain until an asymptote (of maximum stress anisotropy) is reached where it remains fairly constant - with slight fluctuations around the maximum $\sigma_{\mathrm{dev}}^{*}[36]$. The growth rate and the asymptote of $\sigma_{\mathrm{dev}}^{*}$, both increase with $v$.

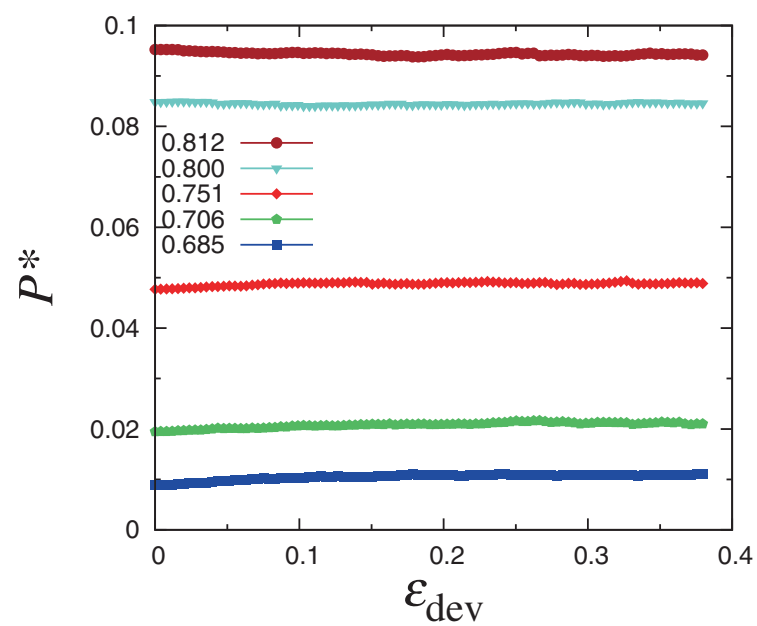

(a)

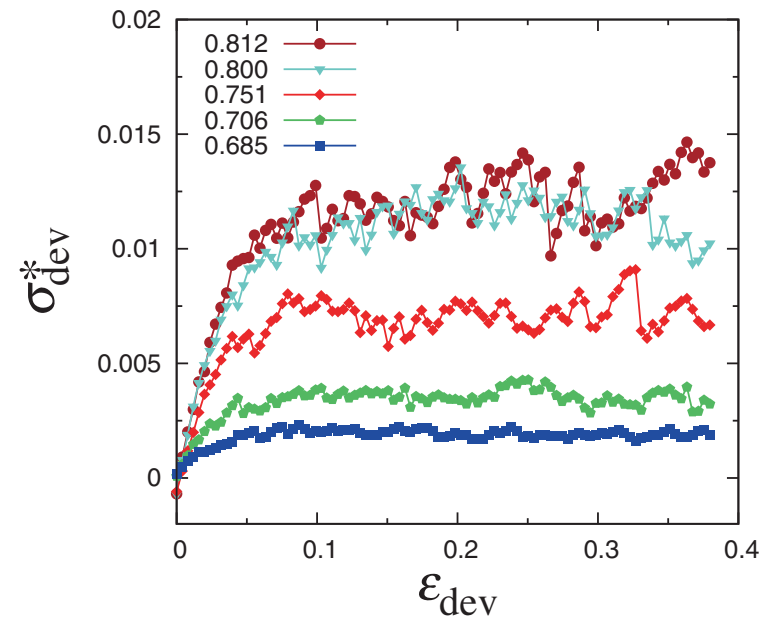

(b)

Figure 4.1: Evolution of non-dimensional (a) pressure $P^{*}$ and (b) shear stress $\sigma_{\mathrm{dev}}^{*}$ along the main strain path for the pure shear deformation mode for five different volume fractions, as given in the inset.

3. We observe a much more pronounced change in pressure when friction is included in the calculation, in agreement with other studies, see e.g. [73]. These data are not shown here and are subject of ongoing research. 


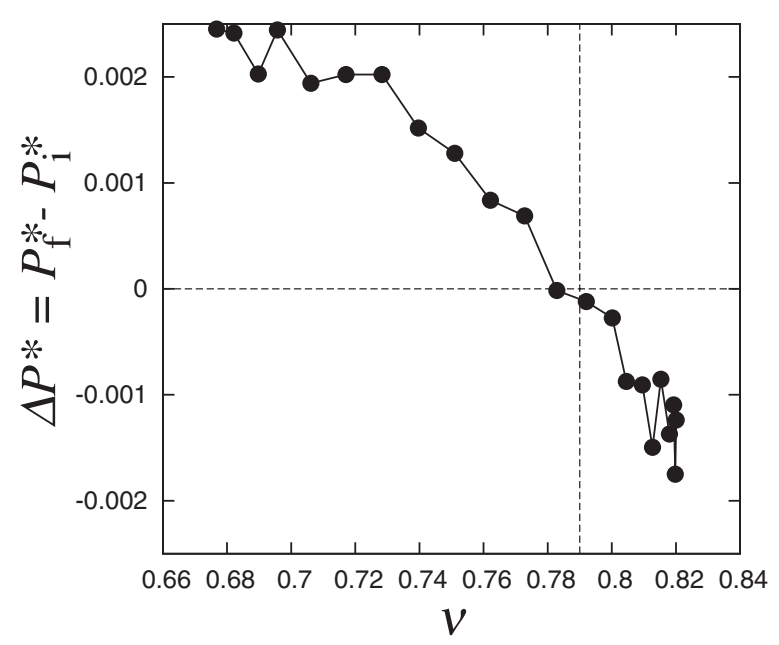

(a)

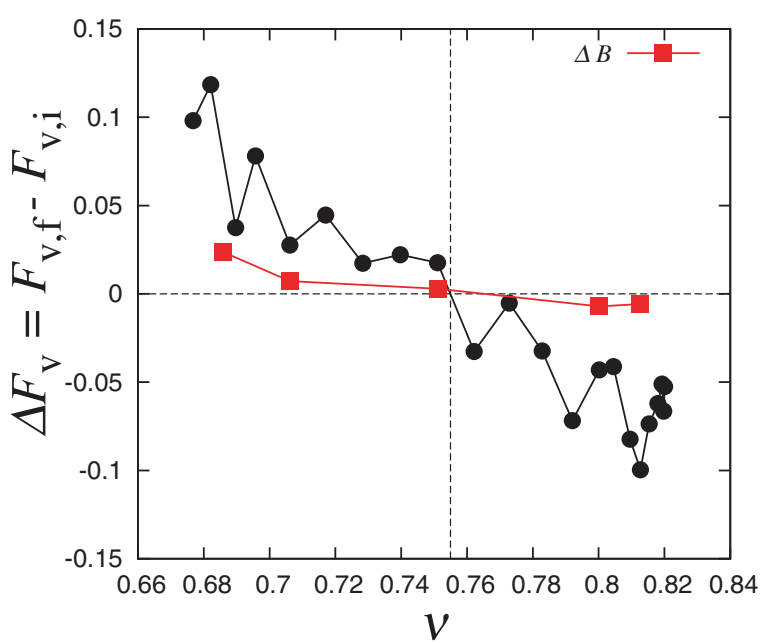

(b)

Figure 4.2: Difference between the final and initial values in (a) non-dimensional pressure $P^{*}$ and (b) isotropic fabric $F_{\mathrm{v}}$ for the pure shear deformation mode for different volume fractions. Red ' $\boldsymbol{\square}$ ' represents the change in bulk modulus, as derived in section 4.4.3. Dashed lines in the plots represent the crossover when these quantities change sign.

\subsubsection{Evolution of fabric}

Complementary to stress, in this subsection we study the evolution of the microstructure in the sample during the volume conserving shear test. Fig. 4.3a shows that the trace of the fabric tensor $F_{\mathrm{v}}$ behaves in a very similar fashion as $P^{*}$, with a slight increase/decrease at the beginning, followed by saturation stage, with magnitude increasing continuously with $v$. Fig. 4.2b shows that the difference between the initial value of $F_{\mathrm{v}}$ and its saturation value, changes sign when a certain volume fraction, $v_{d}^{F}=0.755$, is reached, which is different from $v_{d}^{p}$ for $P^{*}$.

As in Eq. (4.6), $F_{\mathrm{v}}$ is proportional to the product of volume fraction $v$ (that remains unchanged during deviatoric deformations) and coordination number $C$, that varies only little for sheared frictionless systems [84]. Note that as $C=C^{*}\left(1-\phi_{r}\right)$, knowing the (empirical) relations of $C^{*}$ and $\phi_{r}$ with volume fraction, as presented in Refs. [84, 103], we can fully describe the isotropic fabric state. In this study, we assume $F_{\mathrm{v}}$ to stay constant during the shear test; as will be used later in section 4.5 for the prediction of a cyclic shear test. However, the small changes in $F_{\mathrm{v}}$ or $P^{*}$ can be explained be a (small) change in the jamming volume fraction [105].

The evolution of the deviatoric fabric, $F_{\mathrm{dev}}$, as function of the deviatoric strain is shown in Fig. 4.3b during shear for five different volume fractions. It builds up from different random small initial values (due to the initial anisotropy in the sample that develops during prepa- 
ration) to different maximum values. The deviatoric fabric builds up faster at lower volume fractions and the maximal values are higher for smaller volume fractions, qualitatively opposite to the evolution of $\sigma_{\mathrm{dev}}^{*}[36]$.

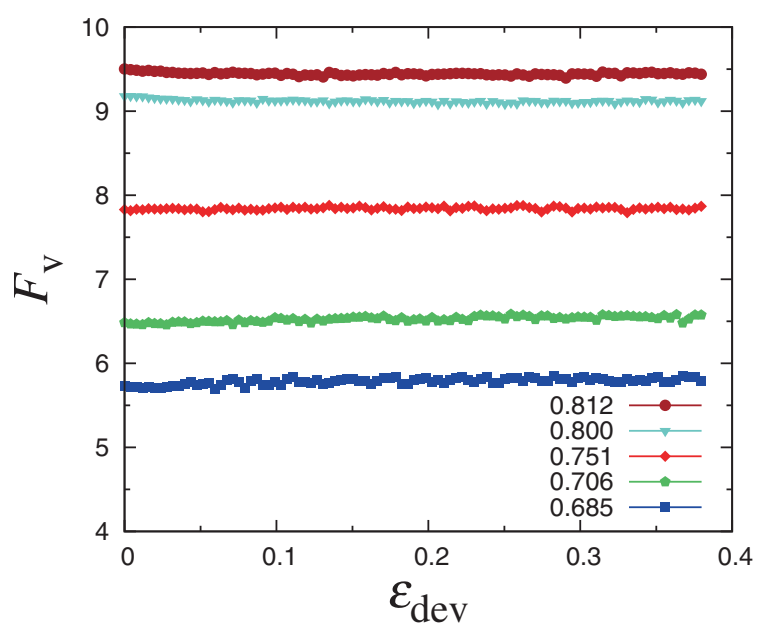

(a)

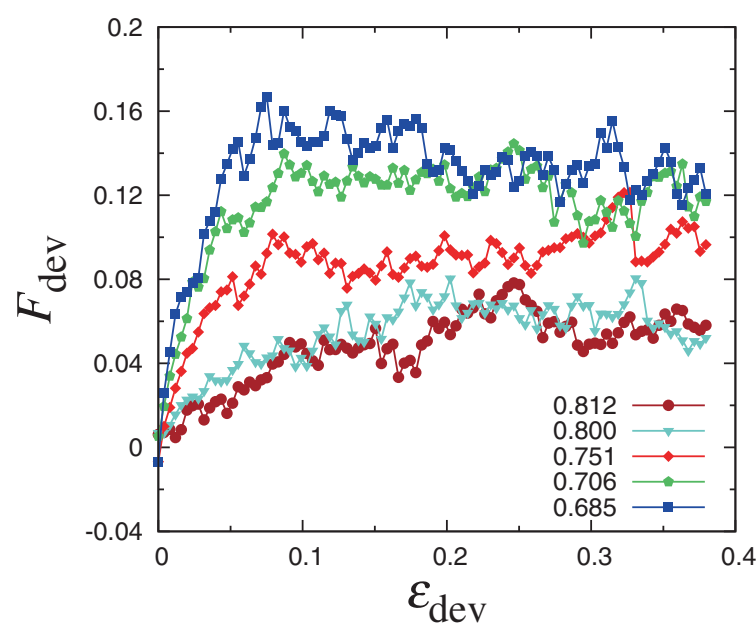

(b)

Figure 4.3: Evolution of (a) isotropic fabric $F_{\mathrm{v}}$ and (b) deviatoric fabric $F_{\mathrm{dev}}$ along the main strain path for the pure shear deformation mode for five different volume fractions, as given in the inset.

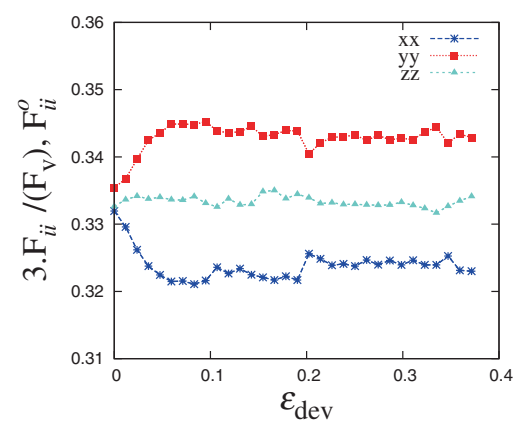

(a)

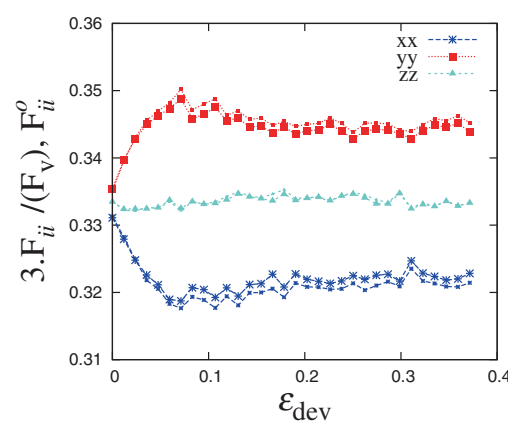

(b)

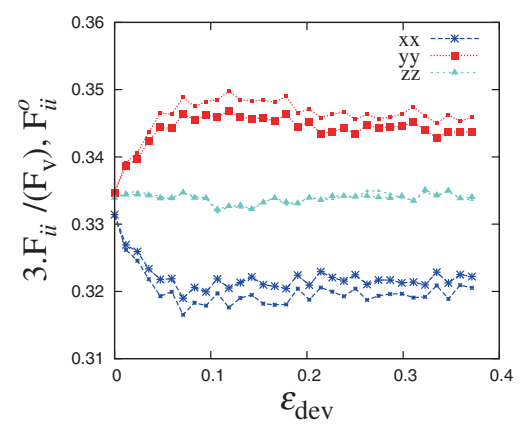

(c)

Figure 4.4: Evolution of the eigen-values of the fabric tensors (directions shown in the inset), during shear deformation at volume fraction $v=0.685$, for the fabric definition defined in Eq. (4.4) (smaller symbols) and the relation presented in Eq. (4.5) (large symbols), for three cases of polydispersity (a) $w=1$, i.e., monodisperse (b) $w=2$ and (c) $w=3$ (present work).

As mentioned in section 4.2.2 the validity of Eq. (4.5), that relates the two different definitions of fabric depends on polydispersity. In order to check the relation, in Fig. 4.4 the evolution of the three eigenvalues of the fabric tensor is plotted, for both definitions, Eqs. (4.3) and (4.4), during the volume conserving shear test, for three different values of polydispersity $w=1,2$ and 3 . For each polydispersity, the chosen low volume fraction $v=0.685$ 
considered here is close to the corresponding jamming points, that depend on $w$, as shown in [103]. The difference between the definitions of fabric becomes higher for higher polydispersity $w=3$, as in Eq. (4.4) the contribution of each particle is weighted to its surface area of the particles, whereas in Eq. (4.3) it is weighted by its volume. For the monodisperse case, the relation is exact, as can be seen in Fig. 4.4a. the differences are considerable for $w=2$ and $w=3$, for compressive and tensile direction, while the non-mobile direction is not affected. We would like to point out that the difference of the two fabrics will be smaller for denser systems.

\subsection{Elastic moduli}

In this section, we first describe the numerical procedure to measure the elastic moduli of the anisotropic aggregate, and later we analyze the elastic data and their relation to the stress and fabric state variables.

\subsubsection{Numerical probes}

In a general framework, we can describe the constitutive behavior of an anisotropic material incrementally as

$$
\left[\begin{array}{c}
\delta P^{*} \\
\delta \sigma_{\mathrm{dev}}^{*}
\end{array}\right]=\left[\begin{array}{cc}
B & A_{1} \\
A_{2} & G^{\mathrm{oct}}
\end{array}\right]\left[\begin{array}{c}
3 \delta \varepsilon_{\mathrm{v}} \\
\delta \varepsilon_{\mathrm{dev}}
\end{array}\right]
$$

where the isotropic and deviatoric components of stress have been isolated and are expressed as functions of $\varepsilon_{\mathrm{v}}$ and $\varepsilon_{\mathrm{dev}}$ via a non-dimensional matrix of moduli (by multiplying the moduli with $k^{*}$, the real stiffnesses can be extracted). $B$ is the classical bulk modulus, and $G^{\text {oct }}$ the octahedral shear modulus. The anisotropy moduli $A_{1}$ and $A_{2}$ provide a cross coupling between the two parts (isotropic and deviatoric) of stress and strain increments. In contrast to the symmetry of the classical stiffness matrix [71], we do not expect the matrix in Eq. (4.9) to be symmetric. This is because the stress and strain vectors have not been chosen in such a way that the product of the stress with the strain increment gives the input work increment [71]. However, we have chosen this kind of representation, since advantages are obtained by investigating the elasticity of a granular material (e.g. soil), not through its resistance to direct stresses expressed by Young's modulus and Poisson's ratio, but rather in terms of stress-response to changes in volume and shape. ${ }^{4}$

To study the evolution of the effective moduli during shear, we choose different initial states (forty) as shown in Fig. 4.5, and apply sufficient relaxation, so that the granular assemblies

4. A third stress, as related to the third invariant, is missing to complete the picture, however, we skip this quantity since its magnitude and changes are always small, for the few example cases we tested it explicitly. 


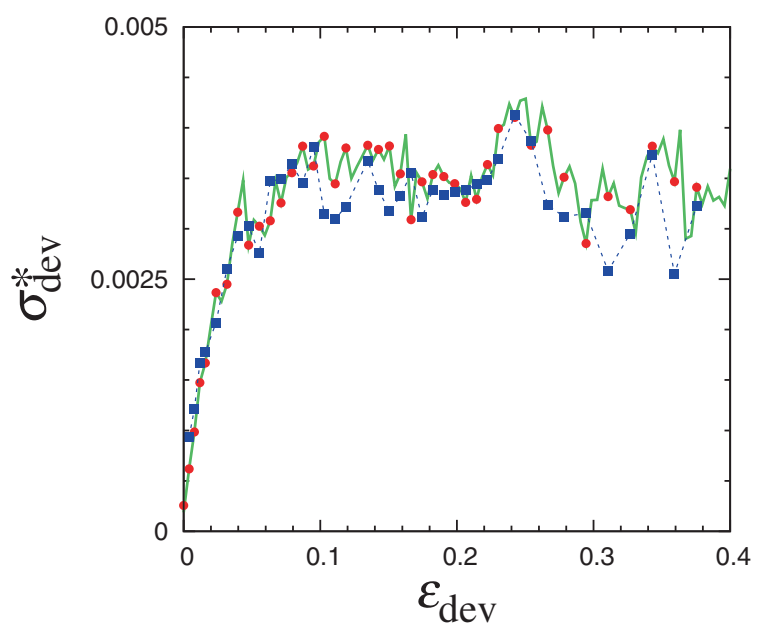

(a)

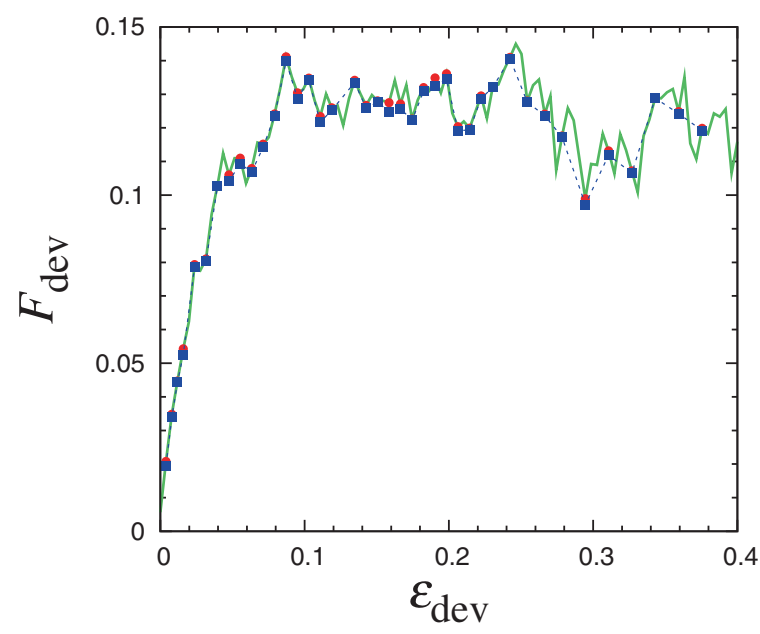

(b)

Figure 4.5: (a) Evolution of non-dimensional shear stress $\sigma_{\mathrm{dev}}^{*}$ (b) $F_{\mathrm{dev}}$, along the main strain path $\varepsilon_{\mathrm{dev}}$ for the pure shear deformation mode, for volume fraction $v=0.706$. The red ' $\bullet$ ' symbols in $(\mathrm{a}-\mathrm{b})$ are the chosen states, which first relaxed (blue ' $\boldsymbol{\square}$ ' symbols in (a) and (b)) and then used as initial configurations for the purely isotropic $3 \delta \varepsilon_{\mathrm{v}}$ and purely deviatoric $\delta \varepsilon_{\mathrm{dev}}$ perturbations.

dissipate the kinetic energy they had during the original shearing path, even though it was very small. Note that, when the states along the shear path are relaxed, the drop in $\sigma_{\mathrm{dev}}^{*}$ is higher than in $F_{\mathrm{dev}}$, as seen in Fig. 4.5. This is because the contact network remains intact leading to no change in $F_{\mathrm{dev}}$, while some of the particle overlaps decrease due to dissipation, leading to drop in $\sigma_{\mathrm{dev}}^{*}$. Then we perform a small strain perturbation to these relaxed anisotropic states, i.e., we probe the samples, and measure the incremental stress response $[104,130]$. Finally, the elastic moduli are calculated as the ratio between the measured increment in stress and the applied strain. We can obtain all the different moduli in Eq. (4.9), by applying an incremental pure volumetric or pure deviatoric strain, measuring the incremental volumetric or shear stress response after, sufficient relaxation to achieve mechanical equilibrium:

$$
\begin{gathered}
B=\left.\frac{\delta P^{*}}{3 \delta \varepsilon_{\mathrm{v}}}\right|_{\delta \varepsilon_{\mathrm{dev}}=0} \quad, \quad A_{1}=\left.\frac{\delta P^{*}}{\delta \varepsilon_{\mathrm{dev}}}\right|_{\delta \varepsilon_{\mathrm{v}}=0}, \\
A_{2}=\left.\frac{\delta \sigma_{\mathrm{dev}}^{*}}{3 \delta \varepsilon_{\mathrm{v}}}\right|_{\delta \varepsilon_{\mathrm{dev}}=0}, \quad G^{\mathrm{oct}}=\left.\frac{\delta \sigma_{\mathrm{dev}}^{*}}{\delta \varepsilon_{\mathrm{dev}}}\right|_{\delta \varepsilon_{\mathrm{v}}=0} .
\end{gathered}
$$

Since the numerical probe experiments are carried out with zero contact friction, we are measuring the resistance of the frictionless material [104], where only normal forces are involved. The first big question concerns the amplitude of the applied perturbation to get the elastic response $[26,61,180]$. 


\subsubsection{How small is small?}

Here, we will discuss the smallness of perturbations amplitude applied to measure the elastic linear response in stress of the granular material and for larger amplitudes the plastic regimes.

\subsubsection{Effect of isotropic perturbations $3 \delta \varepsilon_{\mathrm{V}}$}

Figs. 4.6 (column 1 and 2) show the changes in non-dimensional pressure $\delta P^{*}$, nondimensional shear stress $\delta \sigma_{\mathrm{dev}}^{*}$, isotropic fabric $\delta F_{\mathrm{v}}$ and deviatoric fabric $\delta F_{\mathrm{dev}}$ for different amplitudes of the isotropic perturbation $3 \delta \varepsilon_{\mathrm{v}}$, applied to a relaxed state that has been sheared until $\varepsilon_{\mathrm{dev}}=0.008$ (nearly isotropic configuration: column 1 ) and $\varepsilon_{\mathrm{dev}}=0.38$ (steady state configuration: column 2). Main plots are in log-scale, while the insets are zooms for the small strain region in linear scale. The linear elastic response is also plotted (red solid curve) in the whole strain range, as derived from the incremental behavior for small amplitude, to give an idea of the deviations after large strains.

$\delta P^{*}$ initially increases linearly and smoothly with $3 \delta \varepsilon_{\mathrm{v}}$, in agreement with the linear elasticity prediction. Also the difference between the two initial states (near isotropic and steady state as shown in Figs. 4.6a and 4.6b, respectively) is minimal, meaning that the bulk modulus $B$ (slope of $\delta P^{*}$ with $3 \delta \varepsilon_{\mathrm{v}}$ in the elastic regime) is almost constant and is related to volume fraction or the isotropic part of the contact network tensor, $F_{\mathrm{v}}$, which is mostly unchanged during the shear deformation, as discussed in section 4.4.3. $\delta \sigma_{\mathrm{dev}}^{*}$ behaves similar as $\delta P^{*}$ for small strain, but shows several sharp drops for large strain. These correspond to sudden drops in the coordination number $\delta C^{*}$ (see Fig. 4.7(a-b)), due to rearrangements in the system during the probe. For the nearly isotropic state (Fig. 4.6e), the ratio of $\delta \sigma_{\mathrm{dev}}^{*}$ with $3 \delta \varepsilon_{\mathrm{v}}$ in the linear elasticity regime, i.e. $A_{2}$, is small when compared with the steady state (Fig. 4.6f). This clearly tells that $A_{2}$ evolves during the shear deformation for a given volume fraction, and can be linked with the deviatoric fabric $F_{\mathrm{dev}}$.

$\delta F_{\mathrm{v}}$ increases with $3 \delta \varepsilon_{\mathrm{v}}$, with more fluctuations compared to $\delta P^{*}$, for both states considered here, $\varepsilon_{\mathrm{dev}}=0.008$ (nearly isotropic state, Fig. 4.6i) and $\varepsilon_{\mathrm{dev}}=0.38$ (steady state, Fig. 4.6j), and for every volume fraction (data shown only for $v=0.706$ ). Moreover, the prediction using Eq. (4.6) for $F_{\mathrm{v}}$, matches the dataset very well. $\delta F_{\mathrm{dev}}$ with $3 \delta \varepsilon_{\mathrm{v}}$ stays zero, until the first rearrangement in structure occurs (see Figs. $4.7(\mathrm{c}-\mathrm{d})$ ). After this $\delta F_{\mathrm{dev}}$ starts to decrease with applied $3 \delta \varepsilon_{\mathrm{v}}$, faster in the steady state (Fig. 4.7a) than in the near isotropic state, see Fig. 4.7b. We note here that, when an incremental volumetric strain $\left(3 \delta \varepsilon_{\mathrm{v}}<10^{-6}\right)$ is applied, the system moves from a volume-conserving to a new non-volume-conserving deformation path. As this system is anisotropic, this leads to a decrease $(<0)$ in deviatoric fabric $F_{\text {dev }}$ (opposite to the increase $(>0)$ in deviatoric stress Figs. 4.6e and 4.6f), higher in the steady state (Fig. 4.7b) than in the nearly isotropic state (Fig. 4.7a), meaning that the distance between the volume conserving and non-volume conserving configurations increases with $\varepsilon_{\mathrm{dev}}$. 
nearly isotropic steady state nearly isotropic steady state

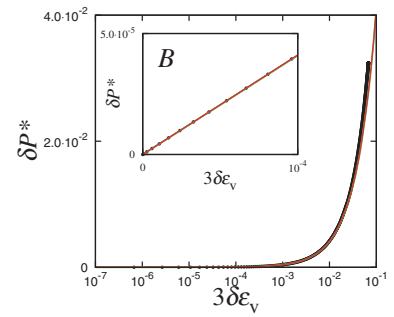

(a)

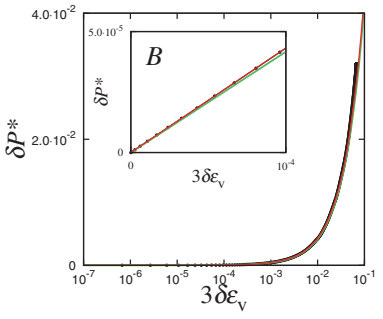

(b)

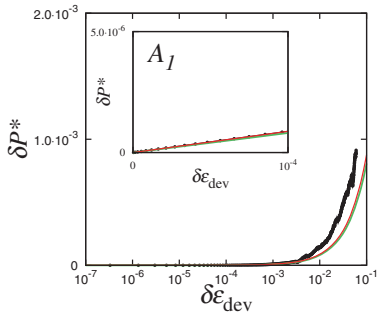

(c)

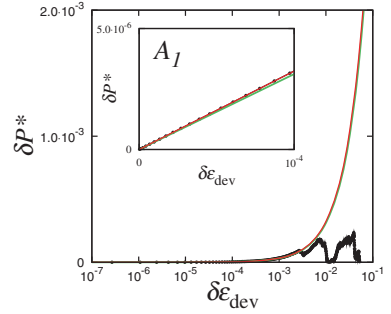

(d)

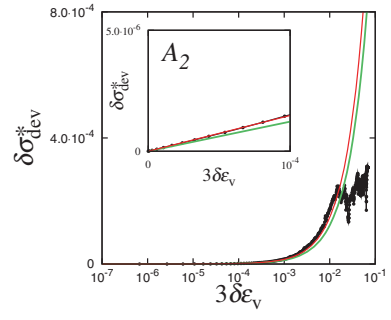

(e)

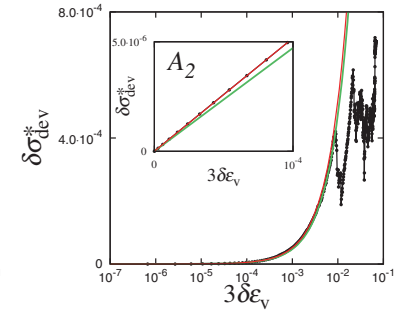

(f)

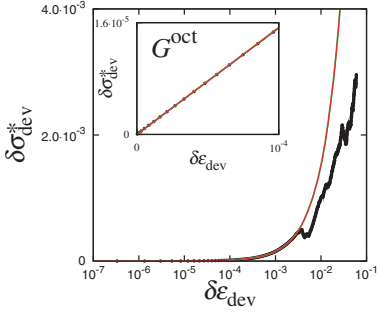

(g)

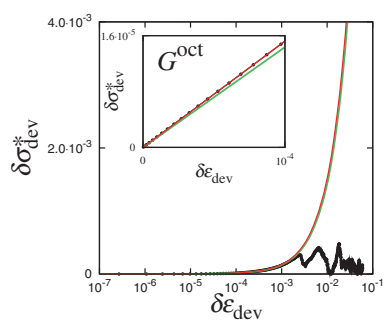

(h)

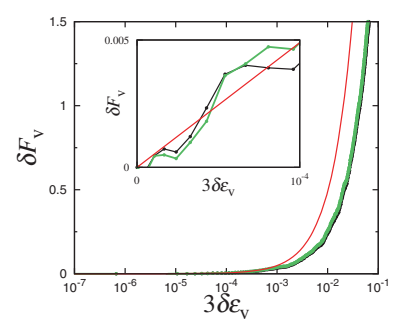

(i)

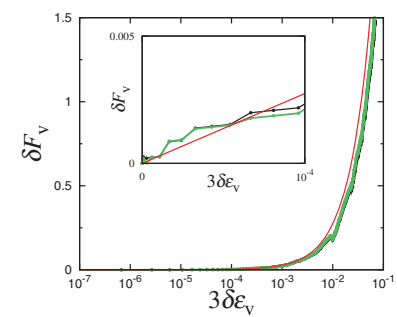

(j)

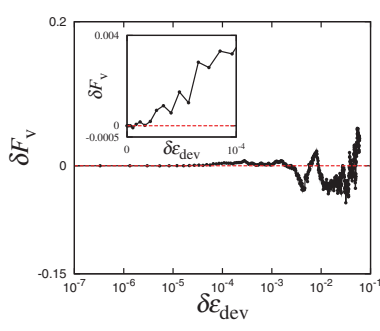

(k)

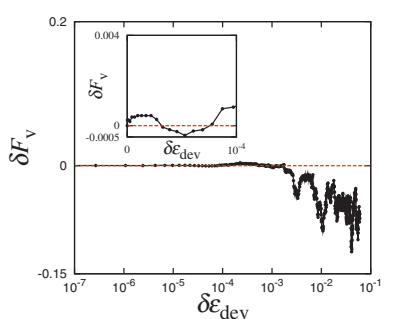

(1)

Figure 4.6: (Rows) Evolution of change in non-dimensional pressure $\delta P^{*}$, non-dimensional shear stress $\delta \sigma_{\mathrm{dev}}^{*}$, isotropic fabric $\delta F_{\mathrm{v}}$ and deviatoric fabric $\delta F_{\mathrm{dev}}$ for different amplitudes. Column 1 and 2 represent purely isotropic while column 3 and 4 represent deviatoric perturbation experiments. The perturbation is applied to the state corresponding to $\varepsilon_{\mathrm{dev}}=0.008$ (nearly isotropic configuration: column 1 and 3) and $\varepsilon_{\mathrm{dev}}=0.38$ (steady state configuration: column 2 and 4 ) of the main deviatoric experiment with volume fraction $v=0.706$. Note that the $x$-axis is log-scale, with inset plots in linear scale. The red line passing through the dataset in (a-j) represents a linear fit in the elastic regime for $3 \delta \varepsilon_{\mathrm{v}} ; \delta \varepsilon_{\mathrm{dev}}<10^{-4}$. The analytical predictions for the elastic range from our results section 4.4.3 in Eqs. (4.11)-(4.15) are plotted as green line in $(\mathrm{a}-\mathrm{h})$. The green line in (i) and (j) represents $F_{\mathrm{V}}=g_{3} v C$ calculated using Eq. (4.6), when subtracted from its initial value. The dashed horizontal line in $(\mathrm{k})-(\mathrm{p})$ represents zero. The green line in $(\mathrm{m})$ and $(\mathrm{n})$ represent the evolution of change in deviatoric fabric $\delta F_{\text {dev }}$ in critical state using parameters from Table 3 of Ref. [84], with the assumption that the new state after volumetric deformation is also in critical state. The green line in (o) and (p) represents Eq. (18) from Ref. [84] when subtracted from its initial value $F_{\mathrm{dev}}^{0}=0.03$ for (o) and $F_{\mathrm{dev}}^{0}=0.113$ for $(\mathrm{p})$, with the growth rate $\beta_{F}=39$ and $F_{\mathrm{dev}}^{\max }=0.12$. 
nearly isotropic

steady state

nearly isotropic

steady state

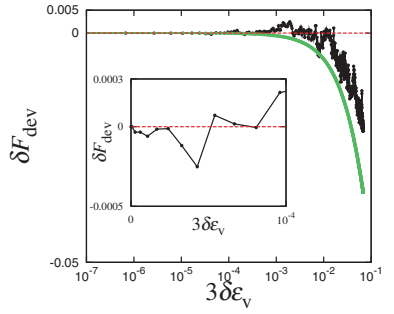

(a)

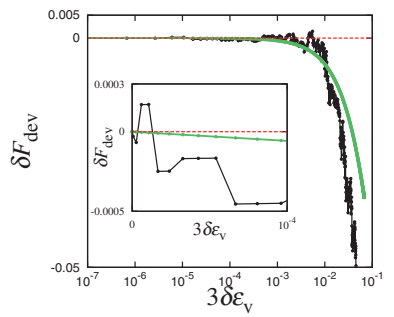

(b)

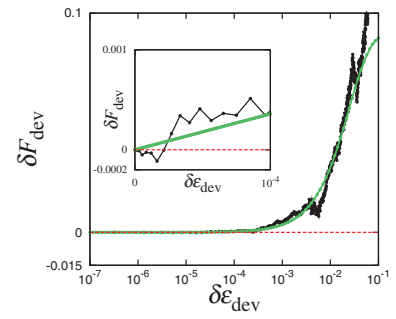

(c)

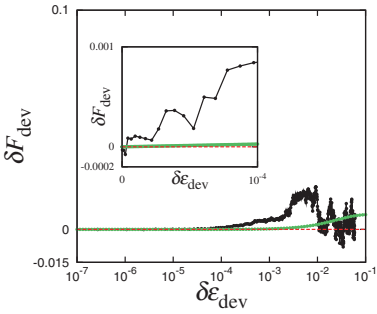

(d)

Fig. 4.6 continued summarizing the parameters for the deviatoric deformation mode.

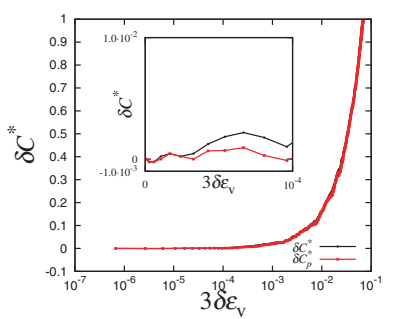

(a)

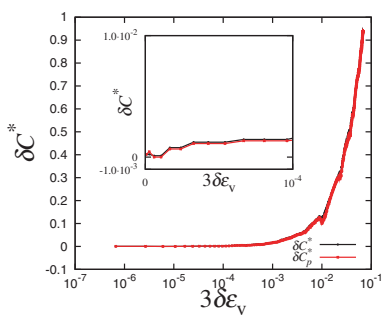

(b)

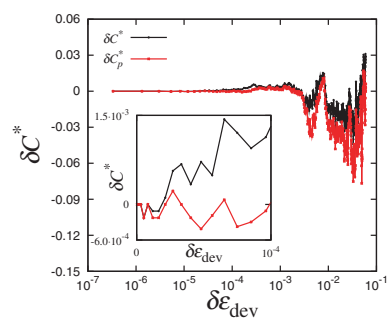

(c)

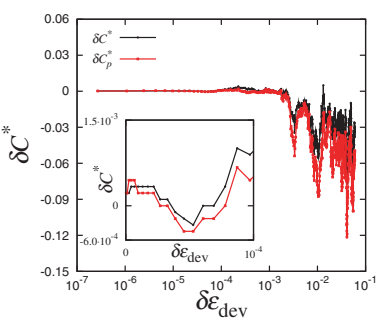

(d)

Figure 4.7: Evolution of change in the coordination number $\delta C^{*}=\delta\left(M_{4} / N_{4}\right)$ (black ' $\bullet$ ' curve) and the modified coordination number $\delta C_{p}^{*}=\delta\left(M_{4}^{p} / N_{4}\right)$ (red '*' curve), defined in section 4.2, during purely $(\mathrm{a}-\mathrm{b})$ isotropic and $(\mathrm{c}-\mathrm{d})$ deviatoric perturbation experiments (corresponding plots as in Fig. 4.6). The perturbation is applied to the state corresponding to $\varepsilon_{\mathrm{dev}}=0.008$ (nearly isotropic configuration: (a) and (c)) and $\varepsilon_{\mathrm{dev}}=0.38$ (steady state configuration: (b) and (d)) of the main deviatoric experiment with volume fraction $v=$ 0.706 . Note that the $x$-axis is on log-scale, with inset plots in linear scale.

Hence, during isotropic compression (increasing $3 \delta \varepsilon_{\mathrm{v}}$ of a pre-sheared (anisotropic) state, both the pressure $P^{*}$ and shear stress $\sigma_{\mathrm{dev}}^{*}$ increase, with pressure increasing much faster leading to a decrease in deviatoric stress ratio $s_{\mathrm{dev}}$. The deviatoric fabric $F_{\mathrm{dev}}$ also decreases with isotropic compression of a pre-sheared state, and the decrease is initially faster than the exponential decay of $F_{\mathrm{dev}}$ (see section 4.5 below) with volume fraction $v$, as seen in Fig. 4.7b. This decrease in $F_{\mathrm{dev}}$ is slower (for large strain) than the exponential decay of $F_{\mathrm{dev}}$, as also seen in Fig. 4.7a. These observations are consistent with the findings of Imole $e t$ al. [84], where the authors noticed a decreasing steady state deviatoric fabric and deviatoric stress ratio with the increasing volume fraction, or $\varepsilon_{\mathrm{v}}$.

\subsubsection{Effect of deviatoric perturbations $\delta \varepsilon_{\mathrm{dev}}$}

Figs. 4.6(column 3 and 4) show the changes in same quantities as before for different amplitudes of the deviatoric perturbation $\delta \varepsilon_{\mathrm{dev}}$, applied to a relaxed state that has been sheared 


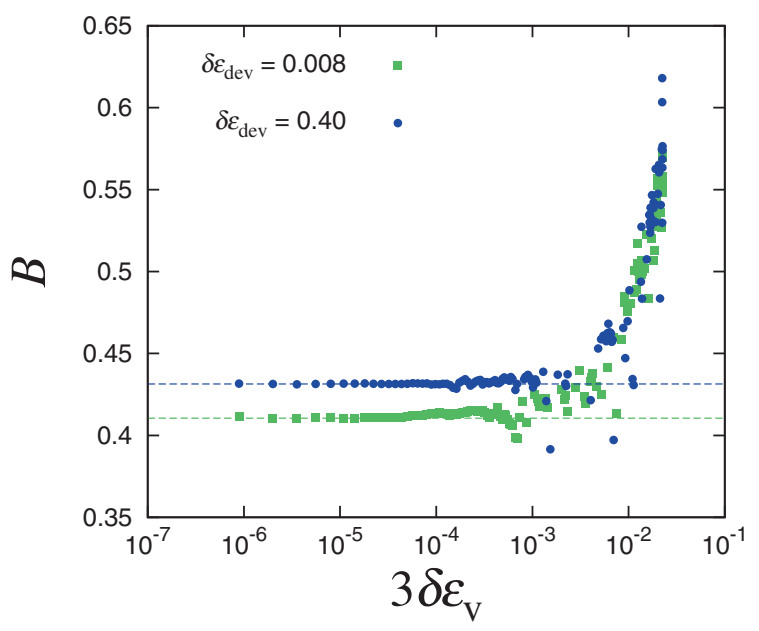

(a)

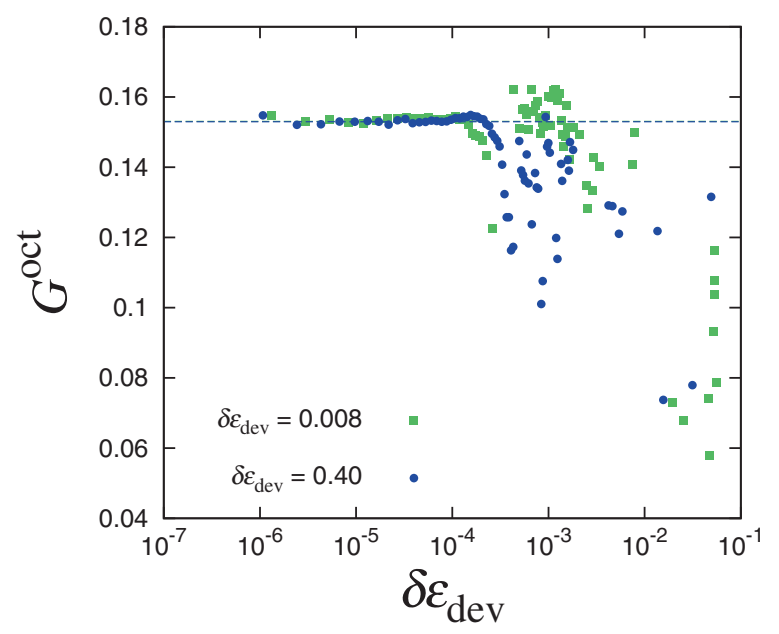

(b)

Figure 4.8: Evolution of (a) bulk modulus $B$ and (b) octahedral shear modulus $G^{\text {oct }}$ with the respective applied isotropic $3 \delta \varepsilon_{\mathrm{v}}$ and deviatoric $\delta \varepsilon_{\mathrm{dev}}$ strain amplitudes for a state corresponding to $\varepsilon_{\mathrm{dev}}=0.008$ (nearly isotropic configuration: green ' $\square$ ') and $\varepsilon_{\mathrm{dev}}=0.38$ (steady state configuration: blue ' $\bullet$ ') of the main deviatoric experiment with volume fraction $v=0.706$. Corresponding dashed horizontal lines represents the initial values of $B$ and $G^{\text {oct }}$.

until $\varepsilon_{\mathrm{dev}}=0.008$ (nearly isotropic configuration: column 3) and $\varepsilon_{\mathrm{dev}}=0.38$ (steady state configuration: column 4).

$\delta P^{*}$ increases linearly with $\delta \varepsilon_{\mathrm{dev}}$ (the slope in the elastic regime is $A_{1}$ ), with $A_{1}$ being much smaller for the nearly isotropic state (Fig. 4.6c) than for the steady state (Fig. 4.6d). This shows that $A_{1}$ evolves during shear deformation, like $A_{2}$, for a given volume fraction, and can also be linked with the deviatoric fabric $F_{\mathrm{dev}}$. Note that the value of $A_{2}$ is higher (about 1.5 times) than that of $A_{1}$. Also, after large deformation, both states show drops in $\delta P^{*}$, which can be linked to the particle rearrangements at large deformation (see Fig. 4.7(c-d)). A non-linear, irregular behavior shows up for $\delta \varepsilon_{\mathrm{dev}}>10^{-4}$, with $\delta P^{*}$ being positive in the case of a loose sample and negative for dense samples (data not shown), in agreement with the observations in Fig. 4.2a. $\delta \sigma_{\mathrm{dev}}^{*}$ also increases linearly with $\delta \varepsilon_{\mathrm{dev}}$ (the ratio in the elastic regime is $G^{\text {oct }}$ ), with $G^{\text {oct }}$ slightly higher for the near isotropic state (Fig. $4.6 \mathrm{~g}$ ) than for the steady state (Fig. 4.6h). Actually, $G^{\text {oct }}$ decreases a little and saturates from the isotropic to the anisotropic state; this effect is more pronounced for states with higher volume fraction (see Fig. 4.13b). Again, similar to $\delta P^{*}, \delta \sigma_{\mathrm{dev}}^{*}$ show drops after large deformations, which can be linked to the particle rearrangements at large deformation (see Fig. 4.7(c-d)) In the steady state, the incremental stresses ( $\delta P^{*}$ and $\delta \sigma_{\mathrm{dev}}^{*}$ ) increase linearly for small strain, as the relaxed configuration on which the perturbation is applied is in a smaller stress state with respect to the main path, see Fig. 4.5a. After the first elastic response, $\delta P^{*}$ and $\delta \sigma_{\mathrm{dev}}^{*}$ fluctuate around zero for larger amplitudes, as seen in Figs. 4.6d and 4.6h, respectively, as no change in stress is expected with increasing deviatoric strain in the steady state. 
$\delta F_{\mathrm{v}}$ stays mostly zero when small $\delta \varepsilon_{\mathrm{dev}}$ is applied for both the near isotropic and steady state configurations (Figs. $4.6 \mathrm{k}$ and 4.61 ). With increasing strain amplitude, $\delta F_{\mathrm{v}}$ increases in the case of a loose sample close to the isotropic state (Fig. 4.6k), and decreases for denser samples (data not shown), in agreement with the behavior in Fig. 4.2b. This tells that indeed there is an evolution of $F_{\mathrm{v}}$ with $\varepsilon_{\mathrm{dev}}$, but it is much smaller compared to that of $F_{\mathrm{v}}$ with $\varepsilon_{\mathrm{v}}$, hence this relation is neglected in the present work. In Fig. $4.7 \mathrm{c}, \delta F_{\mathrm{dev}}$ for the nearly isotropic state, stays zero for $\delta \varepsilon_{\mathrm{dev}}<10^{-4}$, when no rearrangements happen and the behavior is elastic, while it reaches a positive finite value for larger amplitude (the slope of the curve in Fig. 4.3b). This finite value increases with increasing anisotropy (or deviatoric strain state) until it reaches zero in the steady state, where no variation of deviatoric fabric is expected with further applied deviatoric strain (see Fig. 4.7d). When compared to the theoretical predictions from Ref. [84], the simulation data for $F_{\mathrm{dev}}$ match with the predictions, where $F_{\mathrm{dev}}$ increases due to shear for the near isotropic state, and does not change for the steady state simulation. In the steady state, the incremental volumetric fabric $\delta F_{\mathrm{v}}$ in Fig. 4.61 behaves in a similar fashion as $\delta F_{\mathrm{dev}}$, and both behave similar to the stress-increments.

\subsubsection{Discussion and comparison}

Much bigger drops appear in the deviatoric response when the isotropic perturbation is applied. Vice-versa, the fluctuations/drops are much larger in pressure rather than in shear stress, when we deal with deviatoric perturbations. Since we are interested in measuring the purely elastic response of the material, we take care that no rearrangements happen in the system during the numerical probe, that is $3 \delta \varepsilon_{\mathrm{v}}$ and $\delta \varepsilon_{\mathrm{dev}}$ are applied only up to $10^{-5}$ (with very slow wall movement rate $\sim 10^{-6}$,i.e., smaller than for the main large shear strain preparation experiment). It is worthwhile to mention here that we tested our method by applying strain perturbations in opposite directions i.e., $3 \delta \varepsilon_{\mathrm{v}}$ and $-3 \delta \varepsilon_{\mathrm{v}}, \delta \varepsilon_{\mathrm{dev}}$ and $-\delta \varepsilon_{\mathrm{dev}}$. This did not lead to any difference in the elastic response, as long as we stay in the limit of elastic perturbations.

We test the rearrangements argument in Fig. 4.8, by plotting the calculated bulk modulus $B$ and octahedral shear modulus $G^{\text {oct }}$ against the amplitude of the applied isotropic $3 \delta \varepsilon_{\mathrm{v}}$ and deviatoric $\delta \varepsilon_{\mathrm{dev}}$ strain, respectively, for states at $\varepsilon_{\mathrm{dev}}=0.008,0.38$ (nearly isotropic and steady state configurations, respectively) of the main deviatoric experiment with volume fraction $v=0.706$. Both $B$ and $G^{\text {oct }}$ stay practically constant for small amplitudes $\left(3 \delta \varepsilon_{\mathrm{v}} ; \delta \varepsilon_{\mathrm{dev}}<10^{-4}\right)$ and we can assume the regime to be linear elastic [36]. At $3 \delta \varepsilon_{\mathrm{v}} \simeq 10^{-4}$, the first change in the number of contacts happens (Fig. 4.7(a-b)) and $B$ starts to increases non-linearly. Similarly, when $\varepsilon_{\mathrm{dev}} \simeq 10^{-4}$, the first change in the number of contacts happens (Fig. 4.7(c-d)) and $G^{\text {oct }}$ starts to decay. It is interesting to notice that for both $B$ and $G^{\text {oct }}$, the elastic regime shrinks when the volume fraction reduces, going towards the jamming volume fraction (data not shown). A similar modulus can be plotted for fabric as $\delta F_{\mathrm{dev}} / \delta \varepsilon_{\mathrm{dev}}$ that, due to the finite size of the system, is identically zero, until the first rearrangement occurs. 


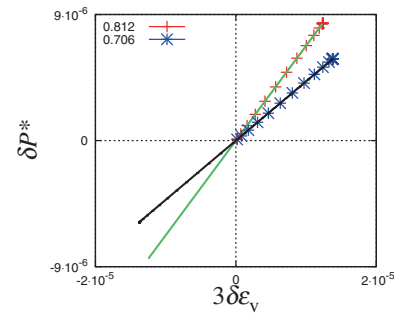

(a)

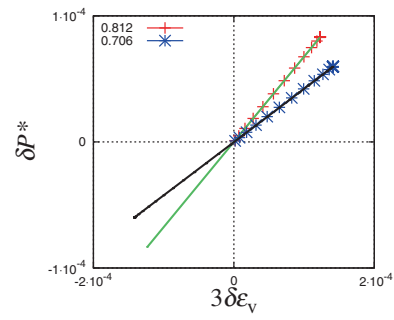

(e)

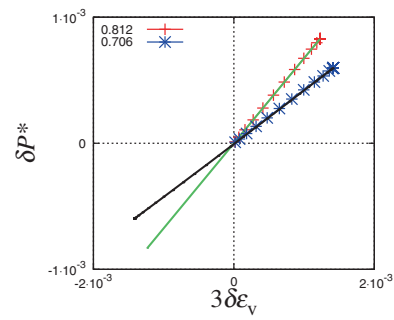

(i)

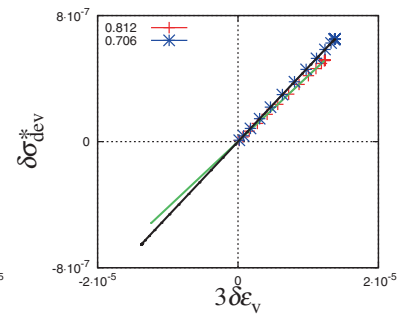

(b)

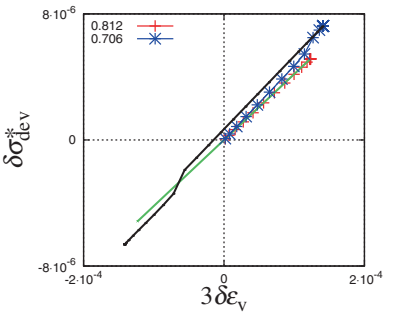

(f)

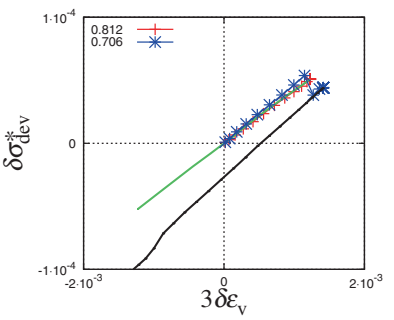

(j)

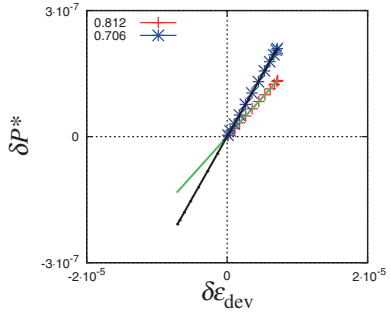

(c)

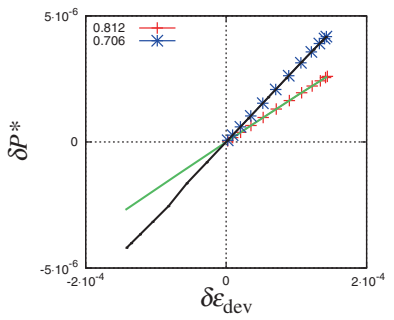

(g)

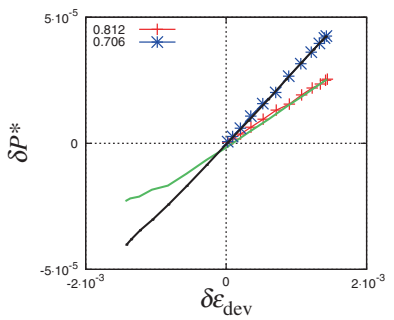

(k)

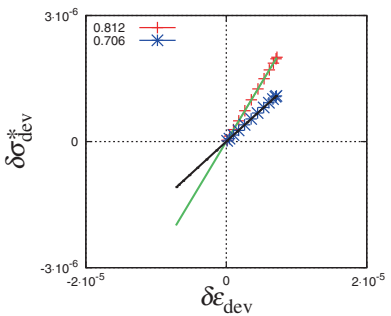

(d)

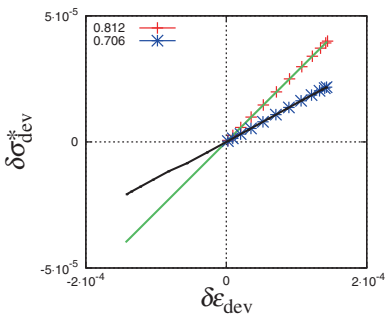

(h)

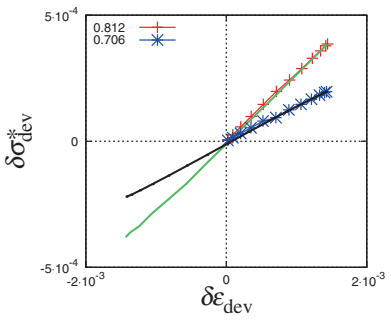

(1)

Figure 4.9: Evolution of non-dimensional pressure $P^{*}$, non-dimensional shear stress $\sigma_{\mathrm{dev}}^{*}$ during small $(\mathrm{a}-\mathrm{d})$, medium $(\mathrm{e}-\mathrm{h})$, and large $(\mathrm{i}-\mathrm{l})$ perturbations in the loading (symbols) and then unloading (solid lines) direction. Red '+' represents loading and the green line represents unloading for $v=0.812$. Similarly, blue '*' represents loading and the black line represents unloading for $v=0.706$. The deformation is applied to the state corresponding to $\varepsilon_{\mathrm{dev}}=0.38$ (steady state configuration) of the main deviatoric experiment.

We further check the elasticity of the probe by reversing the incremental strain. We plot the stress responses to volumetric/deviatoric strain in Fig. 4.9 and compare loading and unloading probes for different volume fractions and amplitudes. Looking at Figs. 4.6, 4.7 and 4.9 together, three regimes seem to appear when perturbations are applied. The first one for very small strain $\left(<5.10^{-6}\right)$, due to the finite size of the system, is characterized by no opening and closing of contacts, and shows perfect reversibility of the data, i.e., elasticity in Figs. 4.9(a-d). The second regime in Figs. 4.9(e-h) shows some weakly irreversible behavior, but only for the smallest volume fraction and a mixed perturbation mode, see the sample at $v=0.706$ in Fig. 4.9f; we associate this behavior to minor contact changes, as visible in Figs. 4.6 and 4.7, but no large scale rearrangements occur. Finally, the third regime, for perturbations two orders of magnitude higher, a residual strain after reversal shows up for both 


\begin{tabular}{c|c}
\hline \hline Modulus & Fit parameter \\
\hline Bulk modulus $B$ & $p_{0}=0.0425, \gamma_{p} \approx 0.2, v_{c}=0.658$ \\
First anisotropy modulus $A_{1}$ & $a_{\mathrm{I}}=0.66 \pm 0.01$ \\
Second anisotropy modulus $A_{2}$ & $a_{\mathrm{II}}=1 \pm 0.02$ \\
Octahedral shear modulus $G^{\mathrm{oct}}$ & $g_{\mathrm{I}}=86 \pm 3$
\end{tabular}

Table 4.1: Summary of fit parameters extracted from the small perturbation results in Eqs. (4.11), (4.12), (4.13), and (4.15).

volume fractions and all types of perturbations, see Figs. 4.9(i-1), proving also that plasticity is much more pronounced in the deviatoric modes than in isotropic ones. We claim that small drops are related to local (weak, almost reversible) re-structuring, while in the last case, the whole system (or big portion of it) is involved in the collapse of the structure, with a more pronounced effect for samples close to the jamming volume fraction [93, 129].

For granular materials, the strain cannot be split in elastic and plastic contributions by "trivially" referring to the residual deformation like in classical solids: as soon as we are out of the elastic range, rearrangements happen during loading and (even though less probably) during unloading, and most likely no original particle position is recovered. Finally, we note that the results shown here are valid for finite-size systems; for much larger (real) samples of much smaller particles, we expect the first elastic regime to reduce to much smaller strains. The boundary between the second and third regime is an issue for further research [171].

\subsubsection{Evolution of the moduli}

Using the four packings at different $v_{i}$, we next determine which variables affect the incremental response of the aggregates at different deviatoric shear strains. In order to understand the role of the microstructure, i.e., the fabric tensor $\mathbf{F}$, the volumetric and deviatoric components, $F_{\mathrm{v}}$ and $F_{\mathrm{dev}}$, are considered as state variables; we postulate that the incremental response of the granular material can be uniquely predicted, once its fabric state (along with the stress state) is known, irrespective of the path that the system experienced to reach that state.

\subsubsection{Bulk modulus $B$}

In Fig. 4.10a, we plot the incremental non-dimensional pressure $\delta P^{*}$ against the amplitude of the applied isotropic perturbation $3 \delta \varepsilon_{\mathrm{v}}$ for one volume fraction, $v=0.706$, and various initial anisotropic configurations. The slope of each line is the bulk modulus in that state. It 


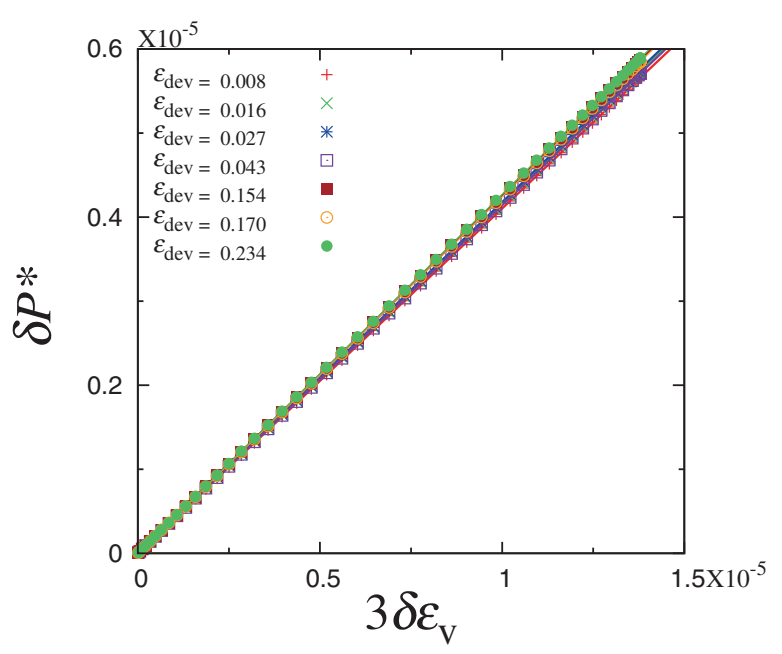

(a)

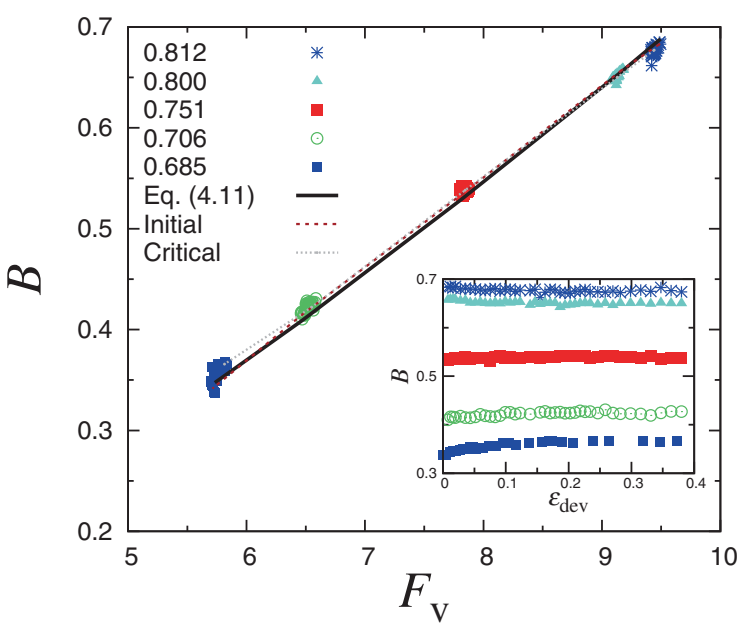

(b)

Figure 4.10: (a) Evolution of change in non-dimensional pressure $\delta P^{*}$ during purely isotropic perturbations $3 \delta \varepsilon_{\mathrm{v}}$ for different states for volume fraction $v=0.706$ along the main path as shown in the inset. (b) Evolution of the bulk modulus $B$ as scaled with isotropic fabric $F_{\mathrm{v}}$ for five different volume fraction as shown in the inset. The solid line passing through the data represents Eq. (4.11). The dashed lines represent the initial and steady state data, as given in the legend.

practically remains unchanged for different states and suggests that $B$ is constant for a given volume fraction.

In Fig. 4.10b, we plot the variation of the bulk modulus $B$, with the isotropic fabric $F_{\mathrm{v}}$ for packings with different volume fractions $v_{i}$. $B$ increases systematically when the five different reference configurations are compared, and it is related to the value of $F_{\mathrm{v}}$ at a given $v_{i}[69,104,178]$. As expected $B$ is a purely volumetric quantity and varies with changes in the isotropic contact network, while the contact orientation anisotropy, $F_{\mathrm{dev}}$, which changes during the main deviatoric deformation path (see Fig. 4.3b) does not affect it much. The inset in Fig. 4.10b shows that the elastic bulk modulus $B$ remains almost constant with the applied shear during the main experiment [104], behaving qualitatively similar to pressure $P^{*}$ and isotropic fabric $F_{\mathrm{v}}$, see Figs. $4.1 \mathrm{a}$ and $4.3 \mathrm{a}$ respectively. In agreement with observations on the volumetric fabric in section 4.3.2, also $B$ shows a slight increase/decrease in the first part of the deviatoric path, more pronounced for loose samples, as clearly seen in Fig. 4.2b. The relation between bulk modulus and fabric was given in Ref. [69] as:

$$
B=\left.\frac{\delta P^{*}}{3 \delta \varepsilon_{\mathrm{v}}}\right|_{\delta \varepsilon_{\mathrm{dev}}=0}=\frac{p_{0} F_{\mathrm{v}}}{g_{3} v_{c}}\left[1-2 \gamma_{p}\left(-\varepsilon_{\mathrm{v}}\right)+\left(-\varepsilon_{\mathrm{v}}\right)\left(1-\gamma_{p}\left(-\varepsilon_{\mathrm{v}}\right)\right) \frac{\partial \ln F_{\mathrm{v}}}{\partial\left(-\varepsilon_{\mathrm{v}}\right)}\right],
$$

where $p_{0}, \gamma_{p}$ and the jamming volume fraction $v_{c}$ are fit parameters presented in Table 4.1. ${ }^{5}$

5. Note that $v_{c}$ for the same particulate system was reported as 0.66 for isotropic deformation in Ref. [69], 
$g_{3} \approx 1.22$ is dependent on the particle size distribution as presented in Refs. [69, 84, 103], see section 4.2. At a known volume fraction, the above relation only requires isotropic fabric $F_{\mathrm{v}}=g_{3} v C=g_{3} v C^{*}\left(1-\phi_{r}\right)$, where the empirical relations for $C^{*}$ and $\phi_{r}$ with volume fraction $v$ are taken from Refs. [84, 103], see section 4.2. The numerical data show good agreement with the theoretical prediction presented in [69] and reported in Fig. 4.10b. The minimum $F_{\mathrm{v}}$ is obtained at the jamming volume fraction, with $v_{c}=0.658, C^{*}=6$, and $\phi_{r}=\phi_{c}=0.13$, leading to $F_{\mathrm{v}}^{\min }=4.2$. At the jamming transition, the bulk modulus $B$ is finite with value $B^{\mathrm{min}}=0.22$. At the jamming transition, $B$ has a discontinuity, having a value zero below $v_{c}[44,86,139,150,153,157,160,218]$.

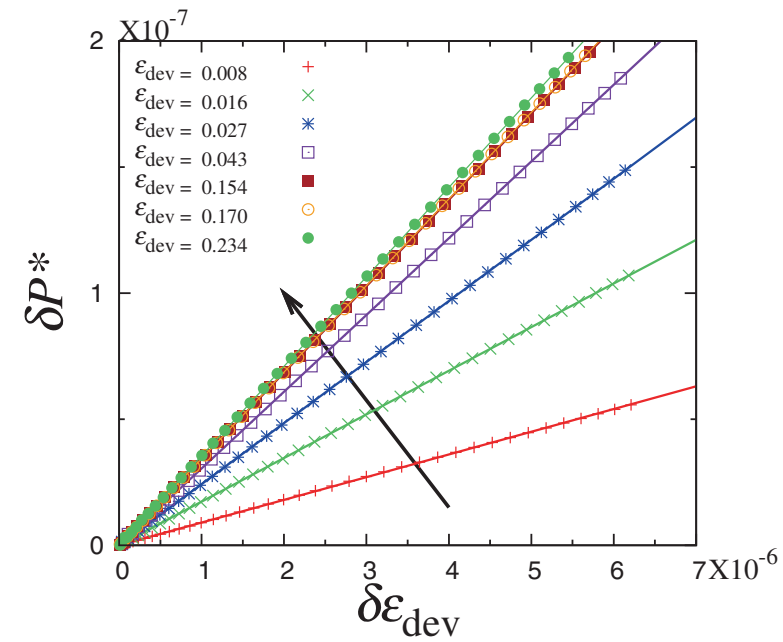

(a)

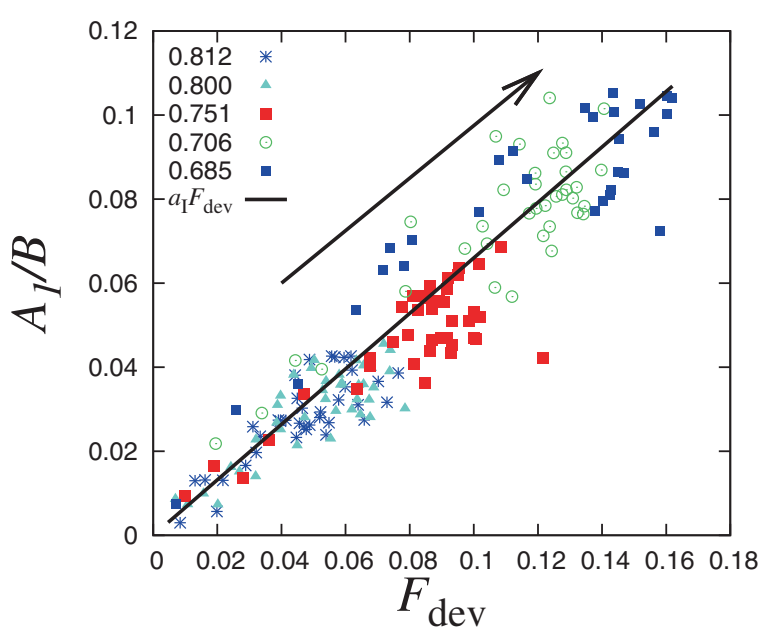

(b)

Figure 4.11: (a) Evolution of change in non-dimensional pressure $\delta P^{*}$ during purely deviatoric perturbations $\delta \varepsilon_{\mathrm{dev}}$ for different states for volume fraction $v=0.706$ along the main path as shown in the inset. The arrow indicates the direction of increasing strain states during main deviatoric experiments. (b) Evolution of the ratio of first anisotropy modulus with bulk modulus $A_{1} / B$ as function of the deviatoric fabric $F_{\mathrm{dev}}$ for five different volume fractions as shown in the inset. The solid line passing through the data represents Eq. (4.12) divided by $B$.

\subsubsection{Anisotropy moduli $A_{1}$ and $A_{2}$}

In Fig. 4.11a, we plot the non-dimensional pressure increment $\delta P^{*}$ against the amplitude of the deformation, when the material is subjected to small deviatoric perturbations $\delta \varepsilon_{\mathrm{dev}}$, to measure the first anisotropy modulus $A_{1}$ as defined in Eq. (4.10), in given anisotropic configurations, as in Fig. 4.10a. Since the material is in an anisotropic state, an increment in

as 0.6646 for isotropic and 0.658 for shear deformation in Ref. [84]. We use a similar $v_{c}=0.658$ here, which, however, is dependent on history of the sample and on the deformation mode. The small deviations of $B$ from Eq. (4.11) can be attributed to a (small) variation of $v_{c}[106]$. 


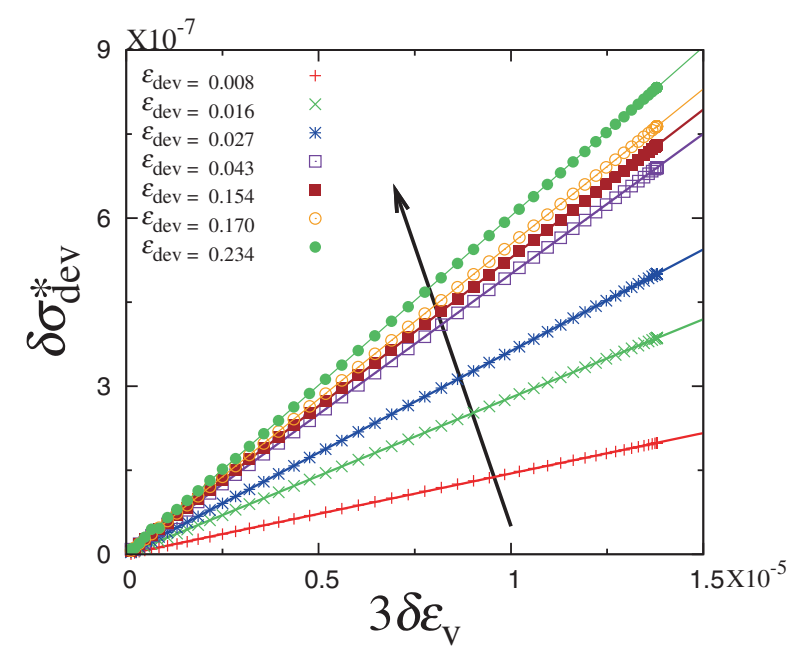

(a)

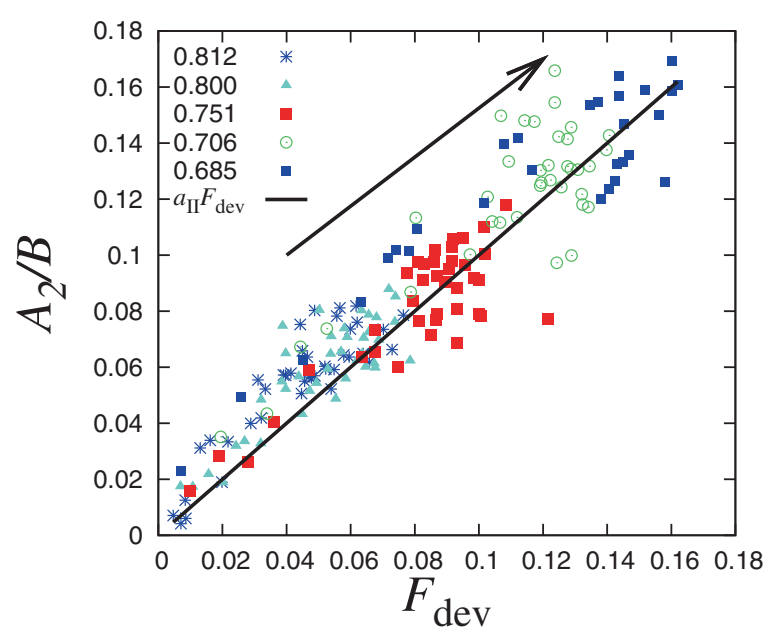

(b)

Figure 4.12: (a) Evolution of change in non-dimensional shear stress $\delta \sigma_{\mathrm{dev}}^{*}$ during purely isotropic perturbations $3 \delta \varepsilon_{\mathrm{v}}$ for different states for volume fraction $v=0.706$ along the main path as shown in the inset. The arrow indicates the direction of increasing strains during main deviatoric experiments. (b) Evolution of the ratio of second anisotropy modulus with bulk modulus $A_{2} / B$ as scaled with the deviatoric fabric $F_{\mathrm{dev}}$ for five different volume fraction as shown in the inset. The solid line passing through the data represents Eq. (4.13) divided by $B$.

deviatoric strain leads to a change in volumetric stress, along with shear stress. The slope of the curves, $A_{1}$, increases with the amount of shear strain the system has experienced, going from small values in the initial isotropic configuration, to an asymptotic limit.

We are interested in the ratio $A_{1} / B$. In this ratio, the dependence of isotropic fabric $F_{\mathrm{v}}$ cancels out, all that remains is a pure dependence on $F_{\mathrm{dev}}$. In Fig. $4.11 \mathrm{~b}$, we plot the variation of $A_{1} / B$, with $F_{\mathrm{dev}}$ for packings with different volume fractions $v_{i}$ as shown in the inset. Besides the fluctuations, the data collapse on a unique curve irrespective of volume fraction and pressure, that is, once a state has been achieved, a measurement of the overall anisotropy modulus is associated with a unique $F_{\mathrm{dev}}$. An increasing trend of $A_{1} / B$ with the fabric factor shows up. As the deviatoric fabric decreases with volume fraction (see Fig. 4.3b), this leads to lower values of the scaled anisotropy modulus for denser systems. In conclusion, we have a linear relation between for the first anisotropy modulus $A_{1}$ :

$$
A_{1}=\left.\frac{\delta P^{*}}{\delta \varepsilon_{\mathrm{dev}}}\right|_{\delta \varepsilon_{\mathrm{v}}=0}=a_{\mathrm{I}} B F_{\mathrm{dev}}
$$

where $B$ is the bulk modulus, $F_{\mathrm{dev}}$ is the deviatoric part of fabric, and $a_{\mathrm{I}} \approx 0.66$ is a fit parameter presented in Table 4.1.

In Fig. 4.12a we plot the stress response of the material $\delta \sigma_{\mathrm{dev}}^{*}$ to isotropic perturbation 
$3 \delta \varepsilon_{\mathrm{v}}$, for the same anisotropic initial configurations as in Fig. 4.10a, to measure the second anisotropy modulus $A_{2}$ as defined in Eq. (4.10). Similarly to $A_{1}$, the slope of the elastic curves, i.e., $A_{2}$, increases with the previous shear strain the system has felt, starting form zero until an asymptotic limit is reached. Again we can relate $A_{2}$ as:

$$
A_{2}=\left.\frac{\delta \sigma_{\mathrm{dev}}^{*}}{3 \delta \varepsilon_{\mathrm{v}}}\right|_{\delta \varepsilon_{\mathrm{dev}}=0}=a_{\mathrm{II}} B F_{\mathrm{dev}}
$$

with $a_{\mathrm{II}} \approx 1$ a fit parameter different form $a_{\mathrm{I}}$ (see Table 4.1). In Fig. 4.12b, we plot the variation of $A_{2} / B$, with $F_{\mathrm{dev}}$ for different volume fractions $v_{i}$ as shown in the inset, where besides the fluctuations, a collapse of data close to Eq. (4.13) is seen. ${ }^{6}$ It is important to note that $A_{2}$ is $\sim 1.5$ times greater than $A_{1}$, confirming the non-symmetry of the reduced stiffness matrix (see section 4.4). This tells that two anisotropy moduli are needed to characterize the volumetric/deviatoric behavior of a three dimensional granular system subjected to a generic (not axial-symmetric) deformation path, in contrast to the single anisotropic modulus $A$ [104], proposed for the 2D case $[128,131]$ and axial-symmetric compression [71].

\subsubsection{Octahedral shear modulus $G^{\text {oct }}$}

In Fig. 4.13a, we plot the shear stress response $\delta \sigma_{\mathrm{dev}}^{*}$ of the material when the initial configurations in Fig. 4.10a are subjected to a small deviatoric perturbation $\delta \varepsilon_{\mathrm{dev}}$. The octahedral shear modulus $G^{\text {oct }}$ is then measured, as defined in Eq. (4.10). The slope of the curves for different initial configurations slightly decreases with the deviatoric state of the system, and saturates for high deformation $\varepsilon_{\mathrm{dev}}$, when the steady state is reached (see Figs. $4.1 \mathrm{~b}$ and $4.3 \mathrm{~b}$ ). That is, the elastic shear resistance of the material decreases when the shear increases for both low and high volume fractions [36]. Interestingly, we find that in this case the deviatoric microstructure alone is not able to capture the variation of the modulus along the shear path, but both stress $\boldsymbol{\sigma}$ and fabric $\mathbf{F}$ seem to influence the incremental shear response, in agreement with findings in [222].

Fig. 4.13 b shows the variation of $G^{\text {oct }}$ against shear strain $\varepsilon_{\text {dev }}$. $G^{\text {oct }}$ starts from a finite value in the initial configuration, related to the isotropic contact network, and slightly decreases with increasing strain, with different rates for different volume fractions. The behavior of $G^{\text {oct }}$ differs from that observed for the bulk modulus in the inset of Fig. 4.10b, as no transition between initial decrease/increase is observed, meaning that a factor other than $F_{\mathrm{v}}$ influences the change of $G^{\text {oct }}$ during the deviatoric path. Similarly to what done for $A_{1}$ and $A_{2}$, we look at the ratio of the shear modulus with the bulk modulus $G^{\text {oct }} / B$ plotted against the isotropic fabric $F_{\mathrm{V}}$ in Fig. 4.13c. The ratio increases with increasing $F_{\mathrm{v}}$, with higher values in the initial state than in the steady state (data are averaged over shear strain $\varepsilon_{\mathrm{dev}} \leq 0.008$ to get

6. A large data scatter is present in both Figs. $4.11 \mathrm{~b}$ and $4.12 \mathrm{~b}$, which increases for increasing deviatoric fabric $F_{\mathrm{dev}}$. This is possibly due to factors that may contribute to the evolution of the anisotropy moduli that are not considered in the present work. 


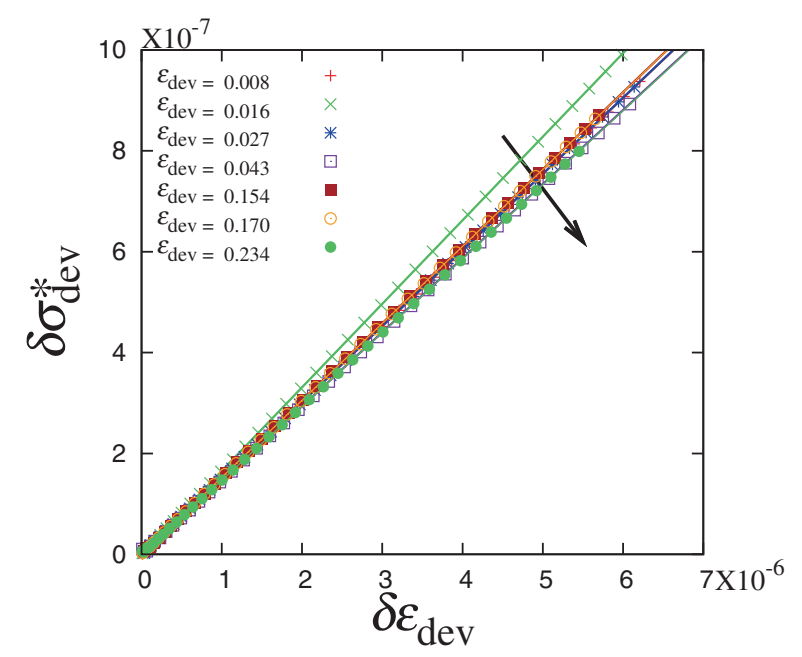

(a)

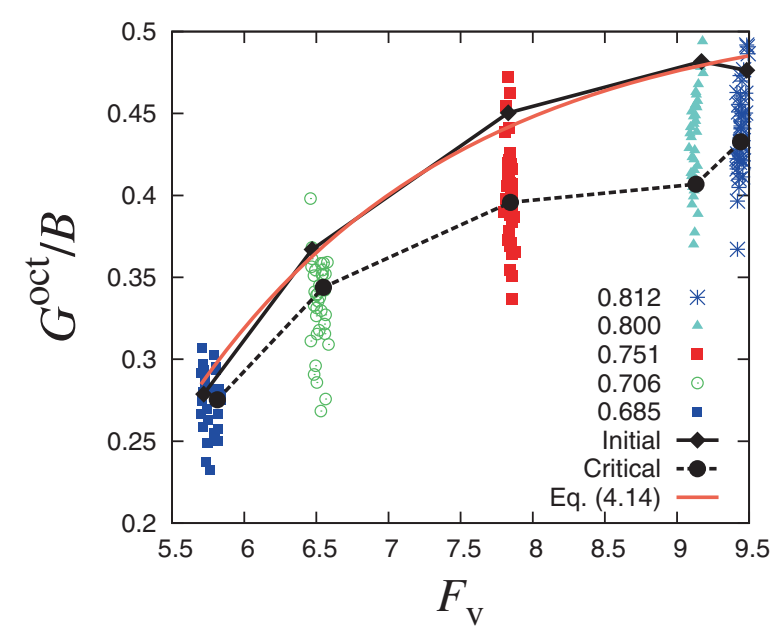

(c)

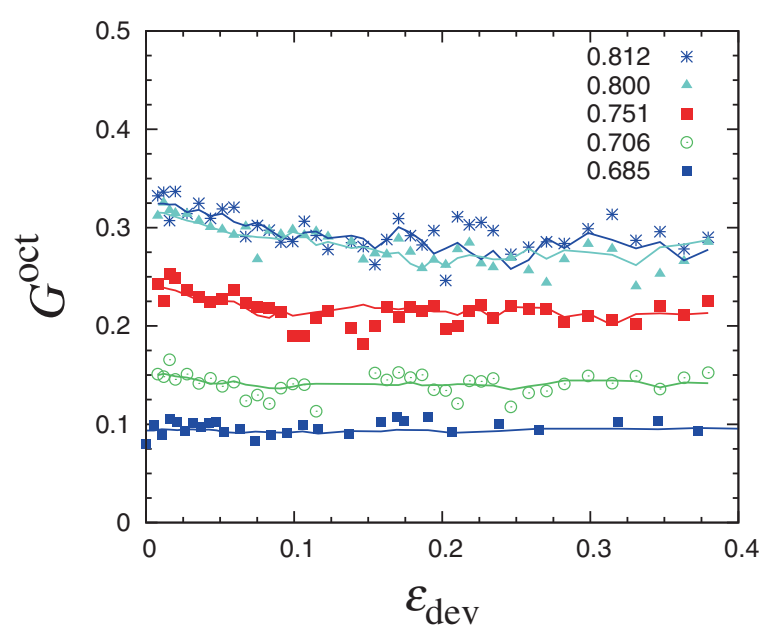

(b)

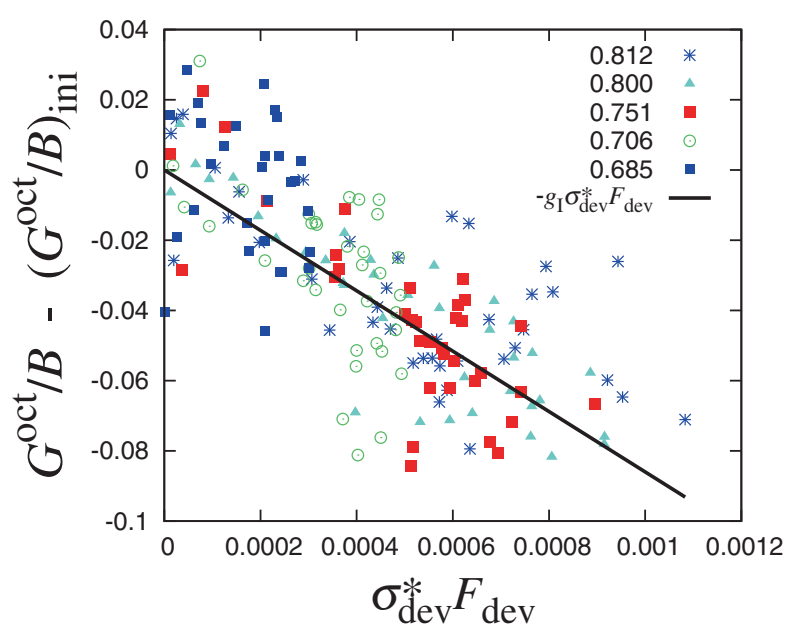

(d)

Figure 4.13: (a) Evolution of change in shear stress $\delta \sigma_{\mathrm{dev}}^{*}$ during purely deviatoric perturbations $\delta \varepsilon_{\mathrm{dev}}$ for different states, with volume fraction $v=0.706$, along the main path as shown in the inset. (b) Evolution of octahedral shear modulus $G^{\text {oct }}$ along the main deviatoric path $\varepsilon_{\mathrm{dev}}$ for five different volume fractions as shown in the inset. The corresponding lines passing through the data represents Eq. (4.15). (c) Evolution of ratio of octahedral shear modulus and bulk modulus, i.e., $G^{\text {oct }} / B$ with isotropic fabric $F_{\mathrm{v}}$, together with the averaged values at the initial (near isotropic state averaged over shear strain $\varepsilon_{\mathrm{dev}} \leq 0.008$ ) and the steady state (averaged dataset in the steady state), as given in the legend. Note that the difference between initial and steady state increases with denser systems. The solid orange line passing through the isotropic dataset represents Eq. (4.14). (d) Evolution of the ratio of octahedral shear modulus and bulk modulus when its initial value, i.e., $G^{\text {oct }} / B-\left(G^{\text {oct }} / B\right)_{\text {ini }}$ is subtracted, plotted using Eq. (4.15), for five different volume fractions as shown in the inset. 
the initial value and in the steady state to get the final one). The isotropic ratio $\left(G^{\text {oct }} / B\right)_{\text {ini }}$ increases with $F_{\mathrm{v}}$, following the power law:

$$
\left(G^{\mathrm{oct}} / B\right)_{\mathrm{ini}}=\left(G^{\mathrm{oct}} / B\right)_{\max }\left[1-\exp \left(\frac{F_{\mathrm{v}}-F_{\mathrm{v}}^{\mathrm{min}}}{F_{\mathrm{v}}^{\alpha}}\right)\right],
$$

where $\left(G^{\text {oct }} / B\right)_{\max } \sim 0.51$ represents the maximum value of ratio $G^{\text {oct }} / B$ for large $F_{\mathrm{v}}$ (or volume fraction), $F_{\mathrm{v}}$ min $\sim 4.2$ volumetric fabric at the jamming transition, presented in section 4.4.3.1, $F_{\mathrm{v}}^{\alpha} \sim 1.9$ is the rate of growth of $\left(G^{\text {oct }} / B\right)_{\text {ini }}$, when the numerical data is extrapolated to the jamming transition, where $\left(G^{\text {oct }} / B\right)_{\text {ini }}=0$. This is in agreement with previous studies that find the upper limit of 0.5 for the ratio between the shear and bulk moduli [53, 99, $130,186]$. In the limit of high $F_{\mathrm{v}}$, the granular assembly becomes highly coordinated and practically follows the affine approximation that predicts a constant value for the ratio $G^{\text {oct }} / B$ [206]. Here, a qualitatively similar behavior is observed for the values in the steady state, approaching a saturation ratio lower than the isotropic one.

Next, in Fig. 4.13d, we subtract the initial value $\left(G^{\text {oct }} / B\right)_{\text {ini }}$ from $G^{\text {oct }} / B$ and assume that $F_{\mathrm{V}}$ does not change during the deviatoric deformation. Hence, we relate the decrease of $G^{\text {oct }}$ to the deviatoric components of stress and fabric via:

$$
G^{\mathrm{oct}}=\left.\frac{\delta \sigma_{\mathrm{dev}}^{*}}{\delta \varepsilon_{\mathrm{dev}}}\right|_{\delta \varepsilon_{\mathrm{v}}=0}=B\left[\left(\frac{G^{\mathrm{oct}}}{B}\right)_{\mathrm{ini}}-g_{\mathrm{I}} \sigma_{\mathrm{dev}}^{*} F_{\mathrm{dev}}\right] .
$$

where $\sigma_{\mathrm{dev}}^{*}$ is the non-dimensional shear stress, $F_{\mathrm{dev}}$ is the deviatoric fabric and $g_{\mathrm{I}} \approx 86$ is a fit parameter reported in Table 4.1. Two contributions of the fabric to the shear stiffness can be recognized - isotropic and deviatoric. The overall contribution is multiplicative proportional to $B$, due to the isotropic contact network, changing very little with deviatoric strain. In the bracket, the first term gives the resistance of the material in the initial isotropic configuration, whereas the second part only depends on the deviatoric (state) variables and characterizes the evolution of the shear modulus with deviatoric strain. That is, given the initial isotropic configuration, the corresponding $G^{\text {oct }}$ is known [48, 130, 206]; on the other hand, the deviation from isotropic to anisotropic network of such configuration uniquely defines the reduction in the shear stiffness. The joint invariant of deviatoric stress and fabric $\sigma_{\mathrm{dev}}^{*} F_{\mathrm{dev}}$ as proposed in $[195,222]$, able to capture the evolution of the ratio of the elastic moduli along the whole undrained path, not only in the steady state, as seen in Fig. $4.13 \mathrm{~d} .{ }^{7}$ No more relation with volumetric quantities needs to be considered, as the evolution of $\sigma_{\mathrm{dev}}^{*} F_{\mathrm{dev}}$ depends on the volume fraction of the sample $v_{i}$.

Note that when $G^{\text {oct }}$ is plotted against Eq. (4.15) in Fig. 4.13c, a deviation from the fitting law is observed for each volume fraction, showing that extra correction terms might be needed for a more accurate description. This is neglected in this preliminary work. It is interesting to

7. Such a split between isotropic and deviatoric fabric influence applies to this specific deformation path, where the volume is conserved. Additional terms may enter when non volume-conserving deformation paths are considered. A very similar behavior is observed when the definition in Eq. (4.4) is employed for the deviatoric fabric. 
point out that the isotropic fabric has different effects in case of the anisotropy moduli $A_{1}, A_{2}$ and $G^{\text {oct }}$, as in the former two cases $F_{\mathrm{v}}$, through $B$, is multiplied to $F_{\mathrm{dev}}$ and contributes to the growth of the moduli from zero to the asymptotic values, while in the latter case $F_{\mathrm{V}}$ defines mostly the initial values of $G^{\text {oct }}$ via the bulk modulus, but does not affect the further decrease.

In the next section, we use the evolution equation for the fabric as predicted from Eq. (4.6), and the relations between the elastic moduli and the stress and fabric, to predict an independent deformation experiment, namely the cyclic shear deformation, i.e., reverse shear after a large deviatoric strain.

\subsection{Prediction of undrained cyclic shear test}

In this section, the constitutive model is presented, involving the elastic moduli measured and calibrated in section 4.4, and the plastic response of the material under large strain. The model is then used to predict the material response under cyclic shear, involving reversal.

\subsubsection{Calibration: Constitutive Model with Anisotropy}

We introduce here a constitutive model as proposed in Refs. [84, 97, 103, 128, 131], extended to three dimensions, that takes into account the evolution of fabric, independently of stress:

$$
\begin{aligned}
\delta P^{*} & =B 3 \delta \varepsilon_{\mathrm{v}}+A_{1} S_{\sigma} \delta \varepsilon_{\mathrm{dev}} \\
\delta \sigma_{\mathrm{dev}}^{*} & =A_{2} 3 \delta \varepsilon_{\mathrm{v}}+G^{\mathrm{oct}} S_{\sigma} \delta \varepsilon_{\mathrm{dev}} \\
\delta F_{\mathrm{dev}} & =\beta_{F} \operatorname{sign}\left(\delta \varepsilon_{\mathrm{dev}}\right) F_{\mathrm{dev}}^{\max } S_{F} \delta \varepsilon_{\mathrm{dev}}
\end{aligned}
$$

In its simplest form, the model involves only four moduli $B, A_{1}, A_{2}$ and $G^{\text {oct }}$, defined in the previous section in Eqs. (4.11) - (4.15). Due to $A_{1}$ and $A_{2}$, the model provides a cross coupling between the two types of stress and strain in the model, namely the isotropic stress $P^{*}$ and shear stress $\sigma_{\mathrm{dev}}^{*}$ reacting to both isotropic $\left(\varepsilon_{\mathrm{v}}\right)$ and deviatoric $\left(\varepsilon_{\mathrm{dev}}\right)$ strains. $F_{\mathrm{dev}}$ evolves differently from stress in two respects, first there is no (or very little, see Fig. 4.9) crosscoupling and, second, the rate of change with deviatoric strain can be (and in many cases is) different than the respective rate for the shear stress evolution. Note that an additional term (modification) is needed for the incremental evolution of $\delta F_{\mathrm{dev}}$ in Eqs. (4.16), due to the observations from Fig. $4.7 \mathrm{~b}$, where $F_{\mathrm{dev}}$ changes when isotropic deformation $\varepsilon_{\mathrm{v}}$ is applied. This term is more significant in the anisotropic state, than in the nearly isotropic state. However, this is neglected in the present work, and is a concern of future investigations, when arbitrary combinations of isotropic and deviatoric strain are applied.

$S_{\sigma}=S / S_{\sigma}^{I}$, with $S=\left(1-s_{\mathrm{dev}} / s_{\mathrm{dev}}^{\max }\right)$ is a measure of the stress isotropy with normalized shear stress ratio $s_{\mathrm{dev}}=\sigma_{\mathrm{dev}}^{*} / P^{*}$, and $S_{\sigma}^{I}$ is the initial stress isotropy at the start of a new 
deformation direction and/or after relaxation. $1-S_{\sigma}$ is the measure for the probability of plastic events. Similarly, $S_{F}=\left(1-F_{\mathrm{dev}} / F_{\mathrm{dev}}^{\max }\right) / S_{F}^{I}$ is the fabric isotropy, and $S_{F}^{I}$ is the initial fabric isotropy at the start of a new deformation direction and/or after relaxation. $s_{\mathrm{dev}}^{\max }$ and $F_{\mathrm{dev}}^{\mathrm{max}}$ represent the maximum (saturation) values of normalized shear stress ratio $s_{\mathrm{dev}}$ and deviatoric fabric $F_{\mathrm{dev}}$ respectively, and $\beta_{F}$ is the rate of change in $F_{\mathrm{dev}}$ at smaller strains (as in Fig. 4.3b).

It is worthwhile to point out that the definitions of $S_{\sigma}$ and $S_{F}$ are different to those used in Refs. $[128,131]$, due to the scaling by the initial reference value. Both $S_{\sigma}$ and $S_{F}$ can take values between 0 and 1 , in contrast to only $S=S_{\sigma}\left(S_{F}=1\right)$ introduced in [131], that takes values ranging from 0 to 2 . Due to $S_{\sigma}$ and $S_{F}$, the incremental response of the material is purely elastic, after relaxation or at strain reversal, with the elastic moduli evolving, as given by Eqs. (4.11) - (4.15), as functions of the stress and structure states. No extra plastic dissipation, related to the approach to the steady state, affects the reversal stiffness. Due to $S_{\sigma}$ and $S_{F}$, the incremental response of the material in the large-strain steady state $(S=0)$ becomes elastic $(S=1)$, just when the strain is reverted or after relaxation. Due to the dependence of the elastic moduli on the stress/fabric state, the model involves non-linear elasticity in its present form, while plasticity due to rearrangements is entirely associated to $S_{\sigma}$. On the other hand, the equation that describes the evolution of fabric is "purely plastic", as there is no change in fabric $\left(\delta F_{\mathrm{v}}=0\right)$, in the elastic regime, when no rearrangements happen. ${ }^{8}$

Now, we can predict an independent experiment, by using Eqs. (4.16), and the relations for the four moduli $B, A_{1}, A_{2}$ and $G^{\text {oct }}$ with microscopic quantities given by Eqs. (4.11) (4.15) with the numerical scaling factors from Table 4.1 (starting from $B$, we can calculate the other moduli using the ratio). Moreover, three other parameters $s_{\mathrm{dev}}^{\max }, F_{\mathrm{dev}}^{\max }$ and $\beta_{F}$ are needed to fully solve the coupled Eqs. (4.16). The dependence of $s_{\operatorname{dev}}^{\max }, F_{\mathrm{dev}}^{\max }$ and $\beta_{F}$ on volume fraction $v$, is well described by the exponential decay relation proposed in Refs. $[84,103]$, with constant values as given in Fig. 4.14 are used, as the volume is conserved in during the cyclic shear test, discussed next.

\subsubsection{Prediction: (Undrained) cyclic shear test}

We choose an initial isotropic configuration, with volume fraction $v=0.711$ and apply deviatoric (volume conserving) shear for one cycle: loading, unloading and final re-loading,

8. We want to point out here the difference between the non-linear elasticity built up along the main deviatoric path and the incremental elasticity, related to the small perturbations. Lets select two states A-B along the deviatoric path as indicated by points in Fig. 4.5, the incremental measured elastic response (moduli) is different between states $\mathrm{A}$ and $\mathrm{B}$ as it depends on stress and fabric, that is the stiffness matrix in Eq. (4.9), varies non-linearly with $\varepsilon_{\mathrm{dev}}$. On the other hand, when the incremental strain $\delta \varepsilon_{\mathrm{dev}}$ is applied to each state (e.g., A or B), the incremental response is linearly elastic (by definition of incremental) and becomes plastic for high $\delta \varepsilon_{\mathrm{dev}}$, as rearrangements happen and the moduli in that given state go from elastic to plastic. 
to recover the initial box configuration. Fig. 4.14 shows the evolution of pressure $P^{*}$, shear stress $\sigma_{\mathrm{dev}}^{*}$, shear stress ratio $s_{\mathrm{dev}}$ and deviatoric fabric $F_{\mathrm{dev}}$ with deviatoric shear strain $\varepsilon_{\mathrm{dev}}$ for one cycle, compared with the prediction using Eqs. (4.16). Since the initial configuration is isotropic, the shear stress $\sigma_{\mathrm{dev}}^{*}$ and $F_{\mathrm{dev}}$ start from zero and approach saturation values (with fluctuations) at large strains. During reversal, both drop with a soft response from their respective saturation value and decrease with unloading strain, crossing their zero values at different strain levels, and finally reach their steady state with negative signs. This supports the need of independent descriptions for the evolution of stress and fabric. Finally, re-loading is applied to reach the initial box configuration. The qualitative behavior of pressure $P^{*}$ is similar in simulations and model, going from a finite initial value to saturation with much less pronounced variations, since the deformation path is volume conserving. It is also interesting that the final state after the complete cycle, which corresponds to the initial box configuration, is highly anisotropic (non-zero stress $\sigma_{\mathrm{dev}}^{*}$ and deviatoric fabric $F_{\mathrm{dev}}$ ).

Both, the shear stress $\sigma_{\mathrm{dev}}^{*}$ and deviatoric fabric $F_{\mathrm{dev}}$, as well as their soft responses during strain reversal are well predicted by the model. $P^{*}$ increases during loading $\varepsilon_{\mathrm{dev}}$ by $\sim 9 \%$ and saturates at large strains. After reversal, $P^{*}$ drops because of opening and release of contacts and then increases again with unloading strain. Although $P^{*}$ is not quantitatively predicted by Eqs. (4.16), the qualitative behavior is captured by the model, which requires a correction as proposed by Krijgsman and Luding [97]. The concept of a history dependent jamming point, introduced by Kumar et al. [105], is capable of capturing the behavior of $P^{*}$ quantitatively, however, this goes beyond the scope of this study.

Eqs. (4.16) provide a complete set of equations able to fully describe the constitutive behavior of a granular assembly, in terms of stress and fabric. Once the initial state and the deformation path are defined, the evolution of isotropic fabric can be determined (using the coordination number and the fraction of rattlers) along the deformation path. The knowledge of isotropic and deviatoric fabric and the incremental relations in Eqs. (4.16) allow for the definitions of the moduli at each incremental step. Given also the probabilities for the plastic events $\left(1-S_{\sigma}\right.$ and $\left.1-S_{F}\right)$, the coupled system can be solved. That is, the characterization of the initial state is the information needed to fully describe the behavior of the material along a general deformation path, defined in terms of strain, since the incremental evolution equations for both stress and structure are given.

In the case of granular materials, the concept of a (homogeneous) material point in a continuum model is debated and many studies have been devoted to the introduction of a length scale in the constitutive model, starting from the Cosserat brothers, see $[39,135]$ among others. Here we limit ourselves and state that a finite-size system is always needed, in order to calibrate any continuum model. That is, any model interpretation works only between the upper/lower bounds of infinite system and particle scale. When a finite-size system is considered an elastic range can always be detected, such that rearrangements happen (see section 4.4.2) with negligible(tiny) probability for very small strain, and an elasto-plastic frame- 


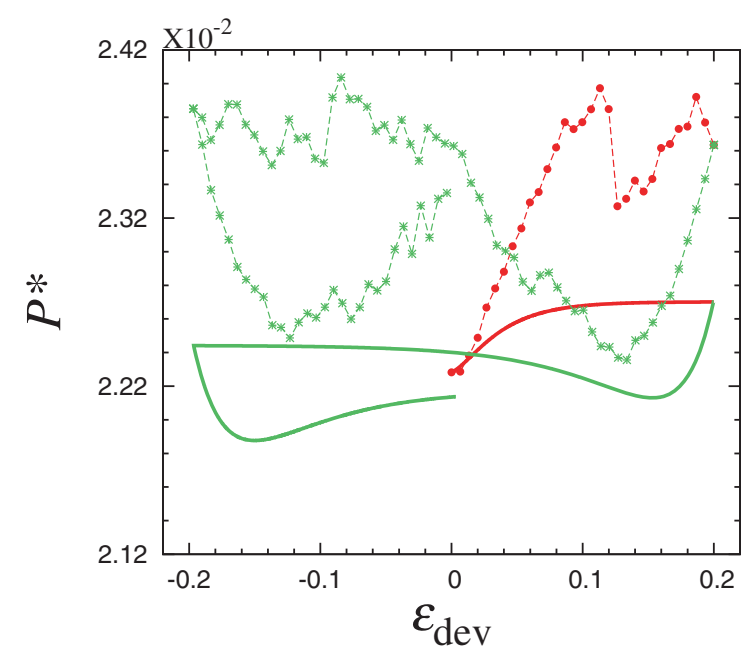

(a)

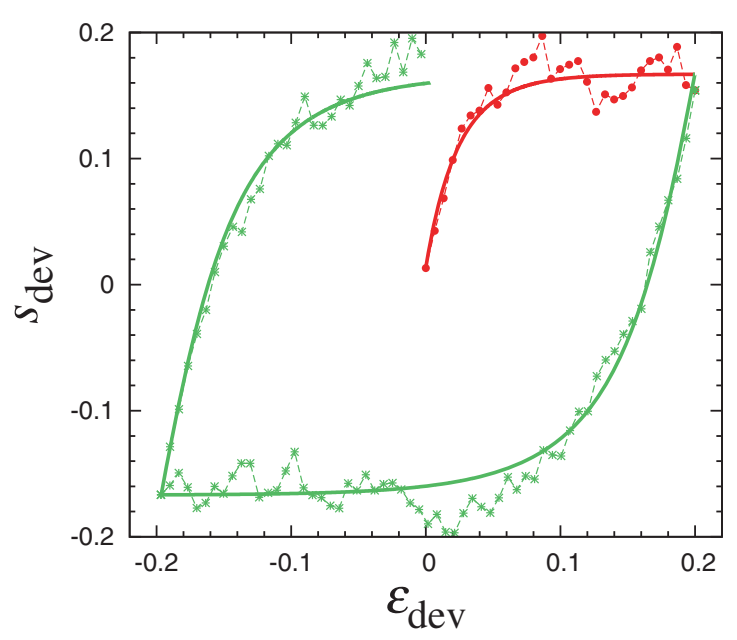

(c)

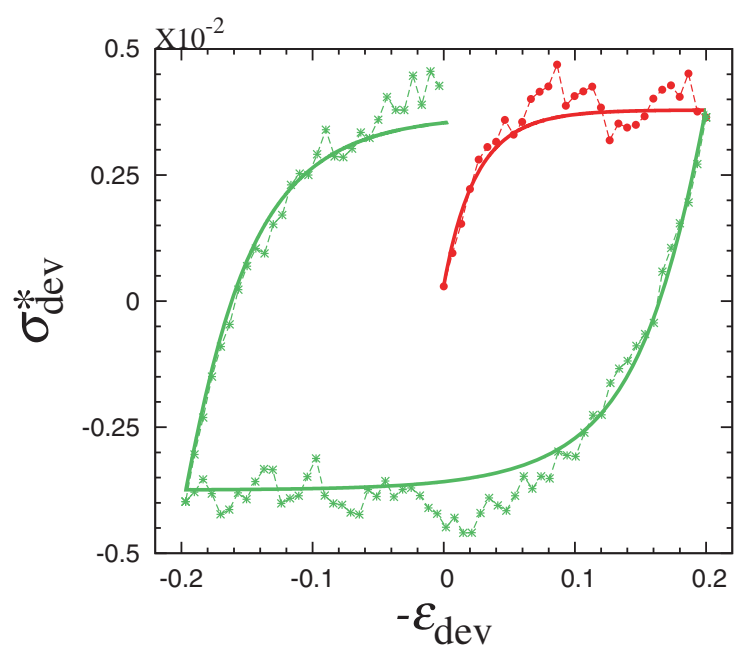

(b)

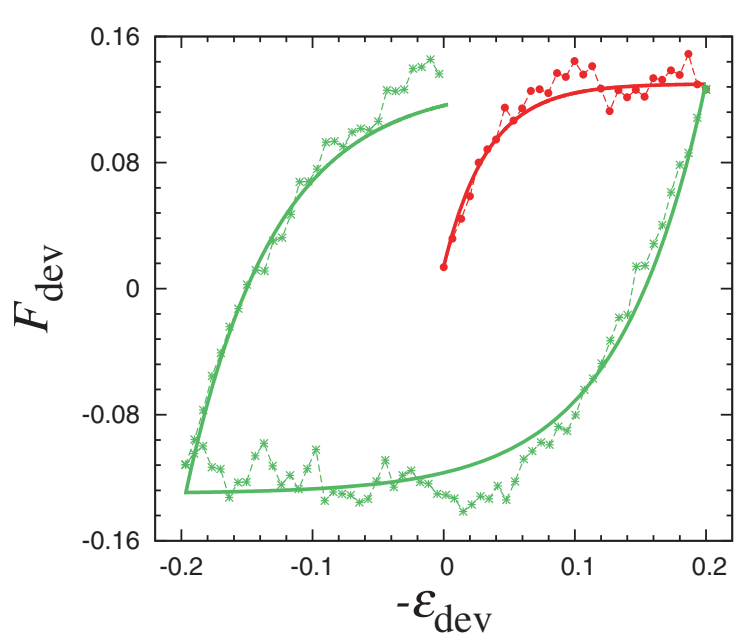

(d)

Figure 4.14: Evolution of (a) pressure $P^{*}$, (b) stress $\sigma_{\mathrm{dev}}^{*}$, (c) normalized stress $s_{\mathrm{dev}}$, and (d) deviatoric fabric $F_{\mathrm{dev}}$ with shear strain $\varepsilon_{\mathrm{dev}}$ during cyclic shear at constant volume $v=0.711$, starting from an initial isotropic configuration. The values of $s_{\mathrm{dev}}^{\max }, F_{\mathrm{dev}}^{\max }$ and $\beta_{F}$ for $v=0.711$ are $0.167,0.124$ and 40.04 respectively, taken directly from the relations proposed in Refs. $[84,103]$. The red ' $\bullet$ ' data points are the DEM simulation data over which the calibration of moduli was done, while the green ' $*$ ' data points represents unloading (reversal) and re-loading. The solid line is the prediction of the DEM observations using Eqs. (4.16).

work could then make sense. Here, we introduce a local rate-type model in Eqs. (4.16), and identify elasticity as the unique initial, static, configuration, from which the (incrementally irreversible) evolution of stress and structure follows. Our choice is to reduce elasticity to a "punctual range", as plastic deformations (which include irreversible opening/closing of contacts by large scale rearrangements) will dominate for large deformations. Dynamics and kinetic fluctuations, leading to relaxation, are not considered here, but also needs to be taken 
into account, see e.g., [90].

\subsection{Summary and Outlook}

In a triaxial box, the four elastic moduli that describe the incremental, elastic constitutive behavior of an anisotropic granular material in terms of volumetric/deviatoric components, namely the bulk modulus $B$, the two anisotropic moduli $A_{1}$ and $A_{2}$ and the octahedral shear modulus $G^{\text {oct }}$, can be measured by applying small strain perturbations to relaxed states that previously experienced a large strain, volume conserving (undrained) shear path. A connection between the macroscopic elastic response and the micromechanics is established, by considering both the stress and fabric tensors, $\boldsymbol{\sigma}$ and $\mathbf{F}$, respectively. While the bulk modulus $B$ depends on the isotropic contact network $F_{\mathrm{v}}$, the deviatoric component of the fabric tensor $F_{\mathrm{dev}}$ is the fundamental state variables needed to properly model the ratios between the (cross-coupling) anisotropic and bulk moduli, i.e., $A / B \propto F_{\mathrm{dev}}$. Hence the two anisotropy moduli $A_{1}$ and $A_{2}$ are related to both deviatoric and isotropic fabric, as the whole contact network determines how the system will react to a perturbation. Surprisingly, when the shear resistance $G^{\text {oct }}$ is considered, both the contact network and the deviatoric stress determine the incremental behavior of the assembly. When the initial response is subtracted, the residual ratio $G^{\text {oct }} / B-\left(G^{\text {oct }} / B\right)_{\text {ini }}$ scales with the deviatoric state of the system, through the product $\sigma_{\mathrm{dev}}^{*} F_{\mathrm{dev}}$. For strain amplitude larger than $10^{-4}$, rearrangements in the sample take place and the behavior deviates from elastic (reversible). The effect of increasing amplitude of isotropic/deviatoric strain perturbations on isotropic/deviatoric stress and fabric is investigated, in the case of nearly isotropic and steady state. For very small strain, the initial (linear) elastic regime, visible in the stress response, is associated to zero change in fabric. For higher strain amplitude applied to nearly isotropic state, plasticity comes in play, and the incremental stress-strain relation deviates from linear as soon as the contact network changes. In the case of steady state, deviatoric strain can only induce fluctuations around the saturation state for both stress and fabric, while large volumetric strain brings the volume-conserved sample on a different strain path.

In the limit of large strain, the tangential moduli of the stress-strain and fabric-strain curves (see Fig. 4.5) are recovered. The relation between particle rearrangements and macro-scale plasticity is a present object of investigation, as well as the transition between local/global plastic regimes.

As further step, a simple constitutive model is introduced that involves anisotropy, as proposed in Refs. [128, 131]. The non-linear elastic behavior is established and the irreversible/ plastic contribution is introduced via empirical probabilities for plastic events, that require more research and theoretical support. The dependence of the model parameters on volume fraction and polydispersity has been analyzed in previous extensive work [84, 103]. Here, by using the new relations for the elastic moduli, we are able to integrate the increments at 
each state along a generic deformation path. Hence we can predict the evolution of pressure, shear stress and fabric for large strain, and also at and after reversal. The method is first calibrated and then applied to a volume conserving (undrained) shear cycle. When the prediction is compared with numerical simulations, quantitative agreement is found for the deviatoric field variables. The most notable feature of soft but different reversal responses of shear stress and fabric are well captured; the pressure response amplitude is underestimated by the present model.

This study concerns a seemingly unrealistic material of spheres without friction and interacting with linear contact forces to exclude effects that are due to contact non-linearity, friction and/or non-sphericity. This allows to unravel the peculiar interplay of stress with microstructure. However, the work should be extended to more realistic cases involving particle shape, friction, and non-linear contact behavior. We expect that friction will not completely change qualitatively the observed relations between stiffness and fabric state, but possibly will add new effects to be explored in the future; the deviatoric fabric and moduli are expected to change quantitatively when tangential forces are included. On the other hand, non-linearity at contacts will introduce an extra pressure-dependence for the moduli, as already shown by many authors (see e.g. [29, 48, 130, 206] in the case of Hertzian interactions). Speculating about the effects of shape goes beyond the scope of this study. A similar analysis is already in progress to check the influence of polydispersity on the relation between elastic stiffness and microstructure, as polydispersity strongly affects the contact network, the structure, and the orientation of contacts [69, 70, 103], see Appendix for more details.

Future work will focus on the extension of our small perturbation approach to elastoplasticity, by using concepts like e.g. the Gudehus response envelope [72, 136]. Other theoretical approaches involve ideas proposed by Einav [52], or by Jiang and Liu [90], for which our results can provide a microscopically based calibration of parameters, but details are not discussed here. The information obtained for the pure elastic range can then be used to decouple the plastic contribution, associated with rearrangements, and to study the flow rule. The validation of the present analysis with experimental data is another important goal. Nevertheless the issue of measuring fabric from laboratory experiments is far from solved, even though big advances have been made in recent years using photoelasticity, and microtomography CT-scans $[21,74,91,188]$. A partial validation is anyway possible when measuring the residual dependence of the elastic response from variables other than stress and porosity [56], by means of acoustic measurements [94]. The behavior after more than one cycle deserves further investigation, from both simulational and theoretical points of view, to detect features like creep, liquefaction and ratcheting, analyzed in preliminary works [131] with constant elastic moduli and for many cycles [105]. Finally, a general tensor formulation that allows for highly different orientations of strain rate, stress and fabric is an open issue but can be inspired by the works of Thornton [195] and Zhao \& Guo [222]. 


\section{A Elastic moduli for polydisperse granular materials}

In this appendix, effect of polydispersity (first on the samples with uniform distribution) will be analyzed, to relate the elastic stiffness with the state variables of the anisotropic material, in order to predict the constitutive behavior along a generic deformation path. Following the methodology shown in Kumar et al. [106], by means of numerical simulation, we apply small perturbations to various equilibrium, relaxed states that previously experienced different pure shear strains and investigate the effect of volume fraction, stress state and microstructure (fabric tensor) on the elastic response of the polydisperse material. Due to the parameter $g_{3}$ that describes the polydispersity of a 3D spherical system, and the jamming point $v_{c}$, we eliminate the effect of polydispersity on the elastic moduli when relating with state variables. A short summary and numerical values of particle parameters used in the DEM simulations is presented in Table 3.1. The sample preparation, relaxation and test procedure is followed the same way as mentioned in section 4.4.2.

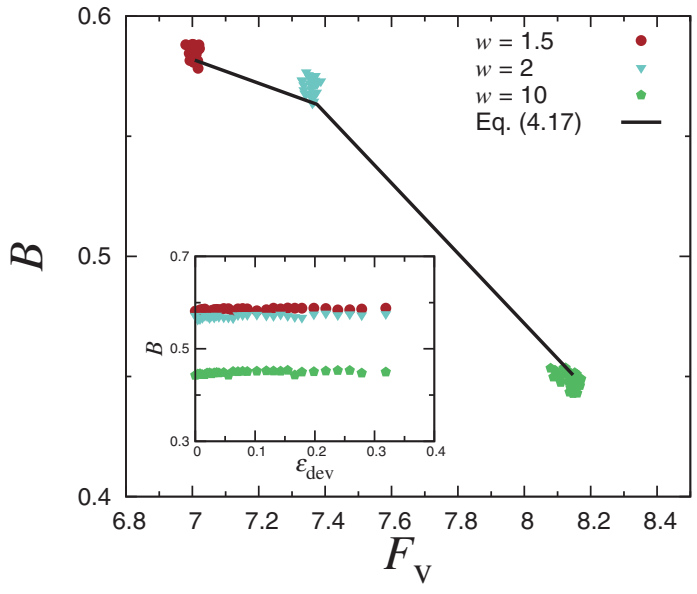

(a)

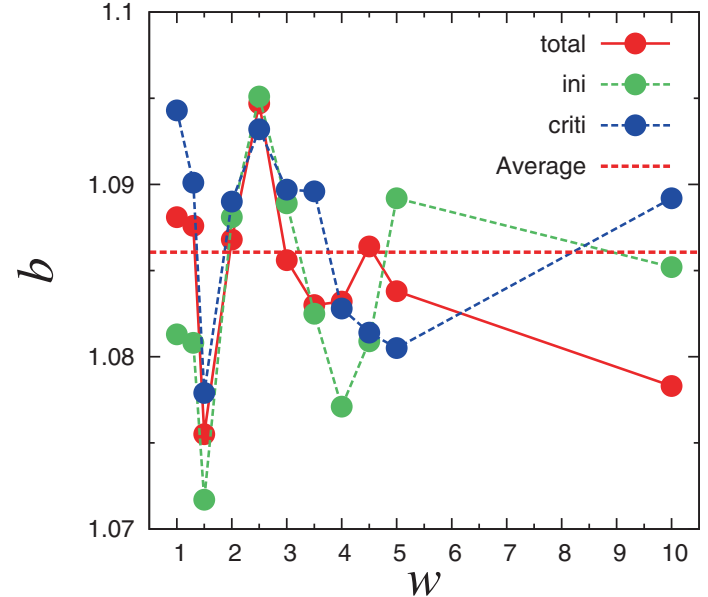

(b)

Figure 4.15: (a) Evolution of the bulk modulus $B$ as scaled with isotropic fabric $F_{\mathrm{v}}$ for states with the same volume fraction $v=0.751$ and different polydispersity as shown in the inset. Solid line passing through the data represents Eq. (4.17). (b) Coefficient $b$ from Eq. (4.17) as function of polydispersity $w$ for fitting in the initial near isotropic, final steady state and the total range as shown in the inset. The horizontal line represents the average of data for the total range.

In Fig. 4.15a, we plot the variation of the bulk modulus $B$, with the isotropic fabric $F_{\mathrm{v}}$ for packings with different polydispersity $w$. The bulk modulus decreases systematically with polydispersity $w$. The relation from Ref. $[69,104]$ is:

$B=\left.\frac{\delta P^{*}}{3 \delta \varepsilon_{\mathrm{v}}}\right|_{\delta \varepsilon_{\mathrm{dev}}=0}=b \frac{p_{0} F_{\mathrm{v}}}{g_{3}(w) v_{c}(w)}\left[1-2 \gamma_{p}\left(-\varepsilon_{\mathrm{v}}\right)+\left(-\varepsilon_{\mathrm{v}}\right)\left(1-\gamma_{p}\left(-\varepsilon_{\mathrm{v}}\right)\right) \frac{\partial \ln F_{\mathrm{v}}}{\partial\left(-\varepsilon_{\mathrm{v}}\right)}\right]$,

where $p_{0}=0.0396$ for the deviatoric mode is taken from Ref. [84, 103]. Jamming point 


\begin{tabular}{c|c}
\hline \hline Modulus & Fit parameter \\
\hline Bulk modulus $B$ & $\langle b\rangle=1.08$ and $p_{0}=0.0425$ \\
First anisotropy modulus $A_{1}$ & $\left\langle a_{\mathrm{I}}\right\rangle=0.62$ \\
Second anisotropy modulus $A_{2}$ & $\left\langle a_{\mathrm{II}}\right\rangle=1.06$ \\
Octahedral shear modulus $G^{\text {oct }}$ & $\left\langle g_{\mathrm{I}}\right\rangle=110$
\end{tabular}

Table 4.2: Summary of parameters extracted from the small perturbation results in Eqs. (4.17), (4.18), (4.19), and (4.20).

$v_{c}(w)$ and parameter $g_{3}(w)$ are dependent on polydispersity $w$ and for the uniform size distribution they are taken from Eq. (3.11) and Eq. (3.8) respectively. $b$ is a numerical value that remains almost constant 1.08 for different polydispersity $w$, as shown in Fig. $4.15 \mathrm{~b}$, and the average value is presented in Table 4.2. At a known volume fraction, the above relation only requires isotropic fabric $F_{\mathrm{v}}=g_{3} v C=g_{3} v C^{*}\left(1-\phi_{r}\right)$, where the empirical relations for $C^{*}$ and $\phi_{r}$ with volume fraction $v$ and polydispersity $w$ are taken from Ref. [103]. The numerical data show good agreement with the theoretical prediction presented in [69] and reported in Fig. 4.10b.

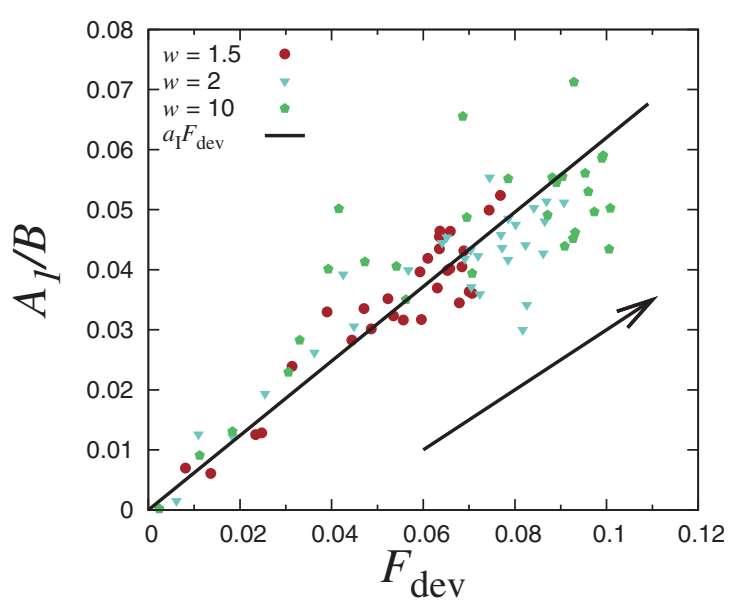

(a)

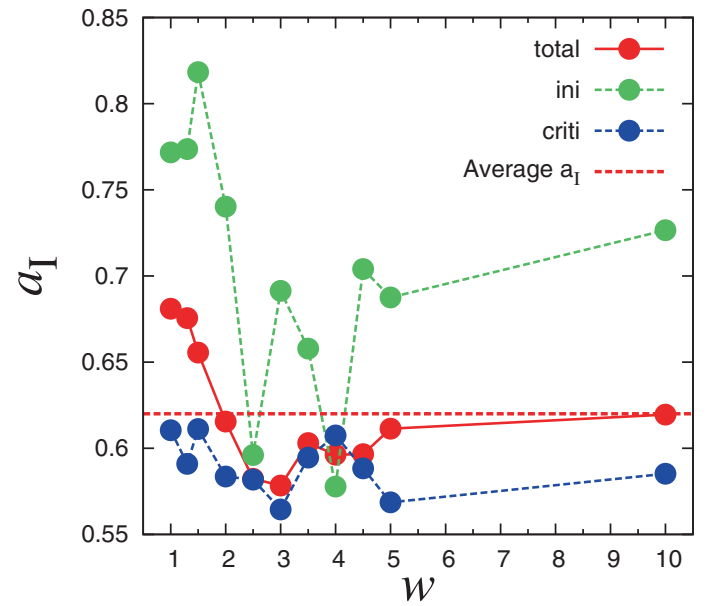

(b)

Figure 4.16: (a) Evolution of $A_{1} / B$ as scaled with isotropic fabric $F_{\text {dev }}$ for states with the same volume fraction $v=0.751$ and different polydispersity as shown in the inset. Solid line passing through the data represents Eq. (4.18). Arrow indicates the direction of increasing

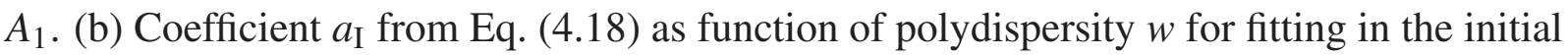
near isotropic, final steady state and the total range as shown in the inset. The horizontal line represents the average of data for the total range. 


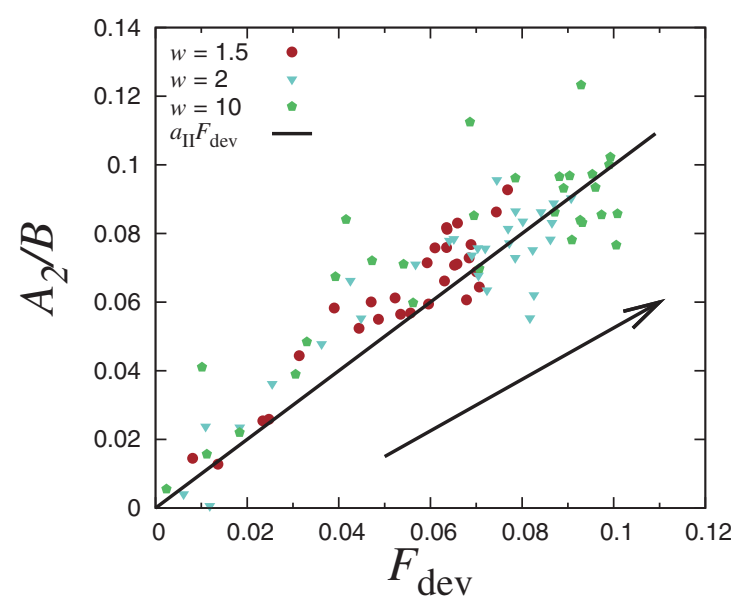

(a)

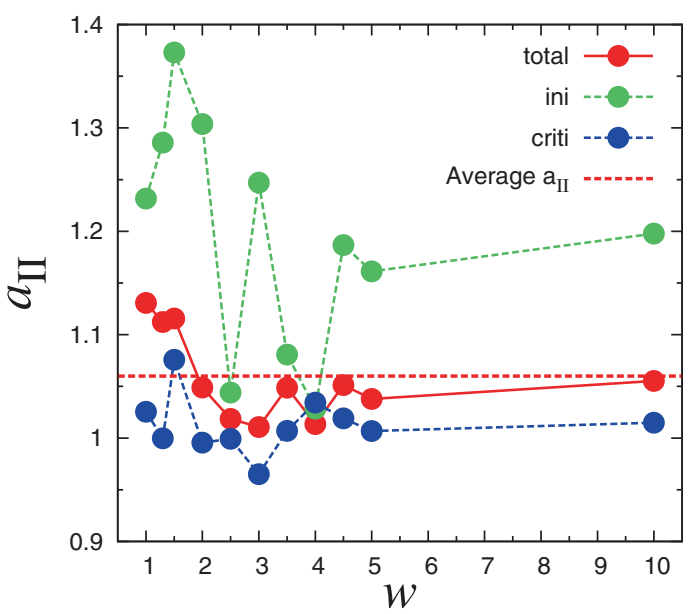

(b)

Figure 4.17: (a) Evolution of $A_{2} / B$ as scaled with isotropic fabric $F_{\text {dev }}$ for states with the same volume fraction $v=0.751$ and different polydispersity as shown in the inset. Solid line passing through the data represents Eq. (4.19). Arrow indicates the direction of increasing $A_{2}$. (b) Coefficient $a_{\mathrm{II}}$ from Eq. (4.19) as function of polydispersity $w$ for fitting in the initial near isotropic, final steady state and the total range. The horizontal line represents the average of data for the total range.

In Fig. 4.16a, we plot the variation of $A_{1} / B$, with the deviatoric fabric $F_{\mathrm{dev}}$ for packings with different polydispersity $w$. Again, an increasing trend of $A_{1} / B$ with the fabric factor shows up for different $w$, and besides the fluctuations, the data collapse on a unique curve irrespective of volume fraction, pressure and polydispersity. In conclusion, we have a linear relation between for the first anisotropy modulus $A_{1}$ :

$$
A_{1}=\left.\frac{\delta P^{*}}{\delta \varepsilon_{\mathrm{dev}}}\right|_{\delta \varepsilon_{\mathrm{v}}=0}=a_{\mathrm{I}} B F_{\mathrm{dev}}
$$

where $B$ is the bulk modulus, $F_{\mathrm{dev}}$ is the deviatoric part of fabric. $a_{\mathrm{I}}$ is a numerical value that remains almost constant 0.62 for different polydispersity $w$, as shown in Fig. 4.16b, and the average value is presented in Table 4.2.

In Fig. 4.17a, we plot the variation of the bulk modulus $A_{2} / B$, with the deviatoric fabric $F_{\mathrm{dev}}$ for packings with different polydispersity $w$. Again, an increasing trend of $A_{2} / B$ with the fabric factor shows up for different $w$, and besides the fluctuations, the data collapse on a unique curve irrespective of volume fraction, pressure and polydispersity. Hence we can relate $A_{2}$ as:

$$
A_{2}=\left.\frac{\delta \sigma_{\mathrm{dev}}^{*}}{3 \delta \varepsilon_{\mathrm{v}}}\right|_{\delta \varepsilon_{\mathrm{dev}}=0}=a_{\mathrm{II}} B F_{\mathrm{dev}},
$$

with $a_{\mathrm{II}} \approx 1.06$ a fit parameter different from $a_{\mathrm{I}} \approx 0.62$, as shown in Fig. $4.17 \mathrm{~b}$, and the average value is presented in Table 4.2. 


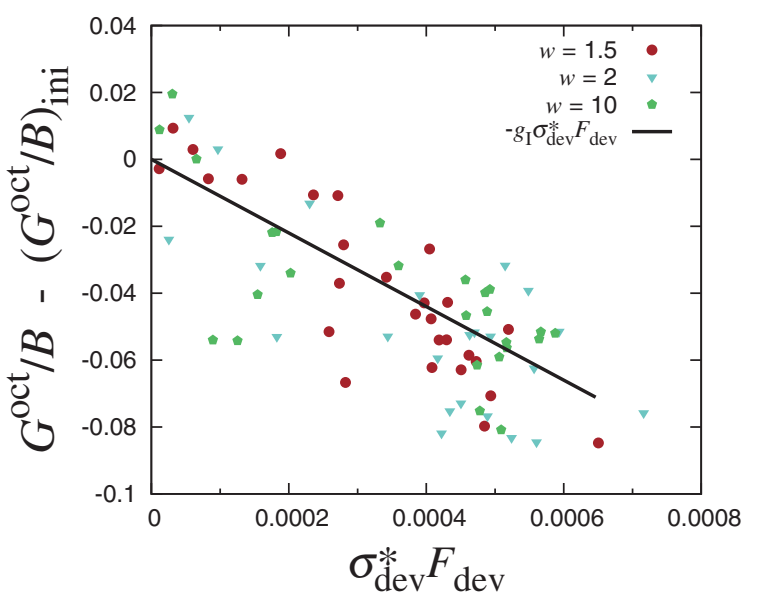

(a)

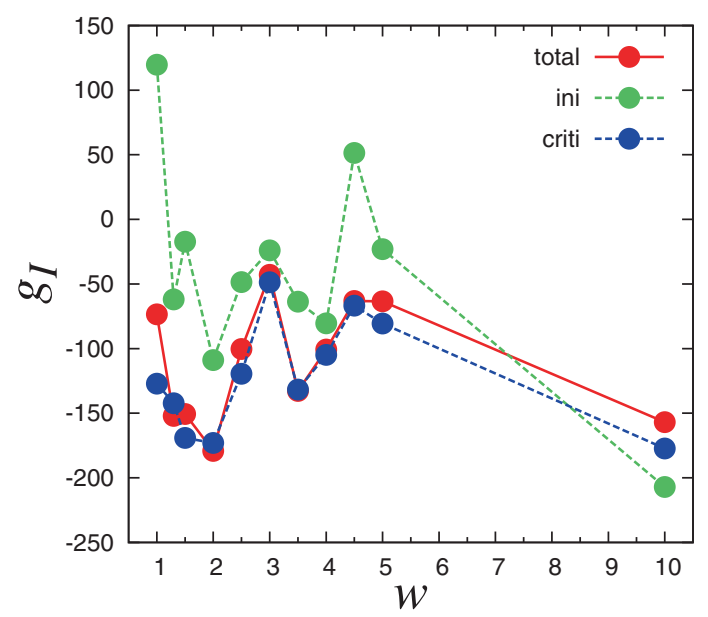

(b)

Figure 4.18: (a) Evolution of the ratio of octahedral shear modulus and bulk modulus when its initial value, i.e., $G^{\text {oct }} / B-\left(G^{\text {oct }} / B\right)_{\text {ini }}$ is subtracted, plotted using Eq. (4.20), for states with the same volume fraction $v=0.751$ and different polydispersity $w$ as shown in the inset.

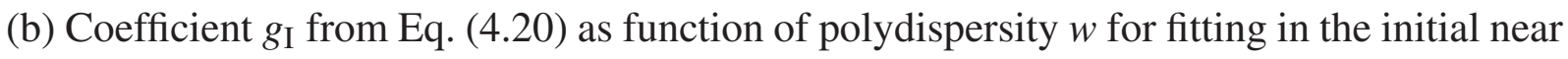
isotropic, final steady state and the total range as shown in the inset. The horizontal line represents the average of data for the total range.

Next, in Fig. 4.18a, we subtract the initial value $\left(G^{\text {oct }} / B\right)_{\text {ini }}$ from $G^{\text {oct }} / B$ and assume that $F_{\mathrm{V}}$ does not change during the deviatoric deformation. Hence, we relate the decrease of $G^{\text {oct }}$ to the deviatoric components of stress and fabric via:

$$
G^{\mathrm{oct}}=\left.\frac{\delta \sigma_{\mathrm{dev}}^{*}}{\delta \varepsilon_{\mathrm{dev}}}\right|_{\delta \varepsilon_{\mathrm{v}}=0}=B\left[\left(\frac{G^{\mathrm{oct}}}{B}\right)_{\mathrm{ini}}-g_{\mathrm{I}} \sigma_{\mathrm{dev}}^{*} F_{\mathrm{dev}}\right] .
$$

where $\sigma_{\mathrm{dev}}^{*}$ is the shear stress, $F_{\mathrm{dev}}$ is the deviatoric fabric and $g_{\mathrm{I}} \approx 110$ is a fit parameter reported in Table 4.2. Two contributions of the fabric to the shear stiffness can be recognized isotropic and deviatoric. The overall contribution is a multiplicative proportional to $B$, due to the isotropic contact network, changing very little with deviatoric strain. In the bracket, the first term gives the resistance of the material in the initial isotropic configuration, whereas the second part only depends on the deviatoric (state) variables and characterizes the evolution of the shear modulus with deviatoric strain. That is, given the initial isotropic configuration, the corresponding $G^{\text {oct }}$ is known $[48,130,206]$; on the other hand, the deviation from isotropic to anisotropic network of such configuration uniquely defines the reduction in the shear stiffness. No more relation with volumetric quantities needs to be considered, as the evolution of $\sigma_{\mathrm{dev}}^{*} F_{\mathrm{dev}}$ depends on the volume fraction of the sample $v_{i}$ and polydispersity $w$. Note that the fit parameter $g_{\mathrm{I}} \approx 110$ fluctuates most when compared to the other elastic moduli. 


\title{
CHAPTER 5
}

\section{Memory of jamming and shear-jamming *}

\begin{abstract}
Understanding the jamming of soft matter as e.g. granular materials, found both in nature and industry, is a challenging fundamental question. Material behaves like a fluid below a certain volume (solid) fraction, known as jamming density or "point", but like a solid or glass above. Various contentious effects and phenomena are reported near this point, most of which are due to changes in the (efficiency of) the packing structure, but a unified picture and model of the dynamics, statistics and rheology of granular materials near jamming is still lacking. Based on the study of three-dimensional soft, frictionless, polydisperse spheres, for the first time, a quantitative model is proposed for how the jamming density changes, using simple isotropic and shear deformation tests. This explains/includes all the previous observations (i.e., that the packing efficiency can increase logarithmically slow under gentle "tapping" or repeated compression), but also includes shear deformations that, in contrast, rapidly decrease the jamming point, which is the only necessary ingredient that explains shear-jamming. All this can be explained by a universal picture involving a multi-scale, fractal-type energy landscape. A key benefit of our work is that the change of the jamming density can be cast into a very simple model, that shall play a significant role in altering constitutive continuum models by adding a memory (history) dependent jamming point, to quantitatively explain the many real-world observations and applications [103].
\end{abstract}

*. Based on N. Kumar and S. Luding. Memory of jamming and shear-jamming. Submitted, 2014 


\subsection{Introduction and Background}

Granular materials are a special case of soft-matter as also foams, colloidal systems, glasses, or emulsions [47, 199]. Granular matter can flow through a hopper or an hour-glass when shaken, but jam (solidify) when the shaking intensity is lowered [207]. These materials jam above a "certain" volume fraction, i.e., the jamming point $[13,21,28,116,117,132,150$, $153,160,181-183,187,200,209,218]$, and become mechanically stable with finite bulkand shear-moduli $[44,86,139,150,153,157,160,218]$. Around the jamming transition, these systems display considerable inhomogeneity, such as reflected by over-population of weak/soft/slow mechanical oscillation modes [182], force-networks [162, 181, 185], diverging correlation lengths and relaxation time-scales [23, 113, 150, 209], and some universal behaviors [154]. Related to jamming, but at all densities, other phenomena occur, like shear-strain localization [153, 158, 174, 200], anisotropic evolution of structure and stress $[21,38,84,150,158,162,174,182,185,209]$, and force chain inhomogeneity [21, 28]. To gain a better understanding of the jamming transition concept, one needs to consider both the structure (positions and contacts) and magnitude of the contact forces. Both of these sets of data illustrate and reflect the transition, e.g., with a strong force chain network percolating the full system and thus making unstable packings permanent, stable and rigid $[21,28,210,220]$.

For many years, scientists and researchers have considered the jamming transition in granular materials to occur at a particular volume fraction, $\phi_{J}$ [24]. In contrast, over the last decade, numerous experiments and computer simulations have suggested the existence of a broad range of $\phi_{J}$, even for a given material. It was shown that the critical density for the jamming transition depends on the preparation protocol [12, 32, 117, 134, 148, 151-155, 198], and that this state-variable can be used to describe and scale macroscopic properties of the system [86]. For example, rheological studies have shown that $\phi_{J}$ decreases with increasing compression rate $[8,134,204,218]$ (or with increasing growth rate of the particles), with the critical scaling by the distance from the jamming point $\left(\phi-\phi_{J}\right)$ being universal and independent of $\phi_{J}[32,33,132,154,221]$ Recently, the notion of an a-thermal isotropic jamming "point" was challenged due to its protocol dependence, suggesting the extension of the jamming point, to become a J-segment [37, 38, 204]. Furthermore, it was shown experimentally, that for a tapped, unjammed frictional 2D systems, shear can jam the system (known as "shear-jamming"), with force chain networks percolating throughout the system, making the assemblies jammed, rigid and stable [21, 163, 210, 220], all highlighting a memory that makes the structure dependent on history $H$. But to the best of our knowledge, quantitative characterization of the varying/moving/changing transition points, based on $H$, remains a major open challenge.

Here, we consider frictionless sphere assemblies in a periodic system, which can help to elegantly probe the behavior of disordered bulk granular matter, allowing to focus on the 
structure, without being disturbed by other non-linearities [21, 77] (e.g. friction, cohesion, walls). For frictionless assemblies, it is often assumed that the influence of memory is of little importance, maybe even negligible. However, we demonstrate its relevance and quantitatively explore its structural origin in systems where the structure and its re-arrangements are the only possible mechanisms leading to the range of jamming points.

In this study, we probe the jamming transition concept by two pure deformation modes: isotropic compression and deviatoric pure shear (volume conserving), which allow us to combine the $J$-segment concept with a history dependent jamming density. Assuming that all other deformations can be superimposed by these two pure modes, we coalesce the two concepts of isotropic and shear induced jamming, and provide the unified model picture, involving a multi-scale, fractal-type energy landscape [100, 117, 214]; in general, deformation (or the preparation procedure) modify the landscape and its population; considering only changes of the population already allows to establish new configurations and to predict their evolution. The observations of different $\phi_{J}$ of a single material require an alternative interpretation of the classical "jamming diagram" [116].

Our results will provide a unified picture, including some answers to the open questions from literature: $(i)$ What happens to the shear-jamming regime in 3D and is friction important to observe it? - as posed by Bi et al. [21]; (ii) What lies in between the jammed and flowing (unjammed) regime? - as posed by Coniglio et al. [37]; (iii) Is there an absolute minimum jamming density? - as posed by Coniglio et al. [37]; (iv) What protocols can generate jammed states?- as posed by Torquato et al. [198]. Eventually, accepting the fact that the jamming density is changing with deformation history, significant improvement of continuum models is expected, e.g., for anisotropic models [84], GSH rate type models [89], or continuum models with a length scale [78]. For this purpose we provide a simple (usable) analytical model that can be used for modifications or generalization of continuum models. Only allowing $\phi_{J}(H)$ to be history dependent, as key modification, explains the multitude of reported observations and can be applied for real-world applications in e.g. electronic industry related novel materials [164].

\subsection{Cyclic isotropic over-compression}

Many different isotropic jamming points can be found in real systems and - as shown here - also for the simplest model material in 3D. We define a jamming "point" as the volume fraction, where the pressure on the unloading branch of an isotropic deformation cycle drops to zero (a cycle means loading and unloading to an unstressed state, (see Appendix Fig. 5.5a). From a relaxed, stress free initial state with volume fraction, $\phi_{t}=0.64<\phi_{J}$, we compress it isotropically to a maximum volume fraction, $\phi_{i}^{\max }$, and decompress back to $\phi_{t}$, and repeat this for $M=100$ cycles. Fig. 5.1a shows the evolution of the isotropic jamming points ${ }^{M} \phi_{J, i}$, which increase with increasing $M$ and with over-compression $\phi_{i}^{\max }$; for subsequent cycles 


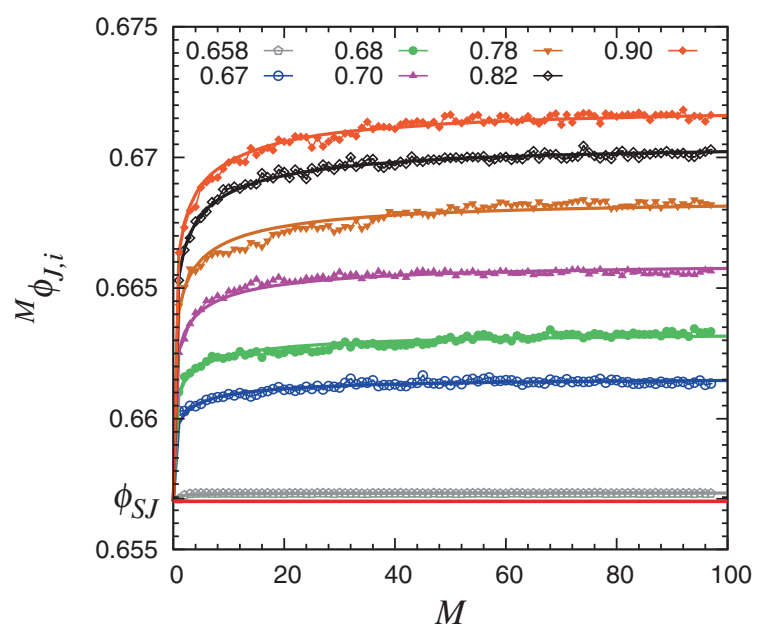

(a)

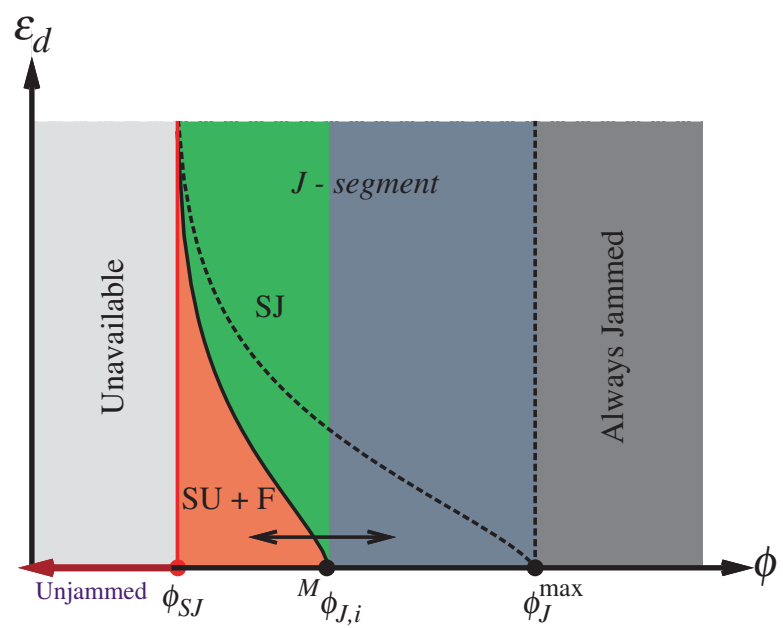

(b)

Figure 5.1: Jamming phase diagram taking into account the changing isotropic jamming points. (a) Evolution of isotropic jamming points ${ }^{M} \phi_{J, i}$ after performing $M$ isotropic compression-decompression cycles up to different maximum volume fractions $\phi_{i}^{\max }$, as given in the inset. With increasing $\phi_{i}^{\max }$, the range of the established jamming points ${ }^{M} \phi_{J, i}=\phi_{J}\left(M, \phi_{i}^{\max }\right)$ increases. The minimum (lower bound) of all ${ }^{M} \phi_{J, i}$ is defined as the shear-jamming limit point, $\phi_{S J}=0.6567$. The solid lines through the data are universal fits to a stretched exponential $[95,165,167]$ with only one single variable parameter $\phi_{J}^{\max }$, i.e., the upper limit jamming point for $M \rightarrow \infty$, which depends on $\phi_{i}^{\max }$ (Appendix 5.C). (b) Schematic jamming phase diagram in volume fraction $\phi-$ strain $\varepsilon_{d}$ space, which includes the shear unjammed (SU), fragile (F) and shear-jammed (SJ) states. Below $\phi_{S J}$, the states are unjammed and application of $\varepsilon_{d}$ does not lead to jamming. Over-compression to very high volume fraction over many cycles $M \rightarrow \infty$, see (a), lets the jamming point $\phi_{J}(H)$ move towards its upper bound of available jamming points, $\phi_{J}^{\max }$, above which the systems are always jammed. The double arrow indicates the movement of $\phi_{J}(H)$, increasing for over-compression and decreasing for shear; the solid black line indicates the shear-jamming transition between (SU+F) and (SJ) states, due to changes in ${ }^{M} \phi_{J, i}$. 
$M$ of over-compressions, the jamming density ${ }^{M} \phi_{J, i}$ grows slower and slower and is best captured by a Kohlrausch-Williams-Watts (KWW) stretched exponential relation :

$$
{ }^{M} \phi_{J, i}:=\phi_{J}\left(\phi_{i}^{\max }, M\right)={ }^{\infty} \phi_{J, i}-\left({ }^{\infty} \phi_{J, i}-\phi_{S J}\right) \exp \left[-\left(M / \mu_{i}\right)^{\beta_{i}}\right],
$$

with the three universal "material"-constants $\phi_{S J}=0.6567$ (Appendix 5.G), $\mu_{i}=1$, and $\beta_{i}=0.3$, the lower limit of possible $\phi_{J}$ 's, the relaxation (cycle) scale and the stretched exponent parameters, respectively. Only ${ }^{\infty} \phi_{J, i}$, the equilibrium (steady-state or shakedown [62]) jamming point limit (extrapolated for $M \rightarrow \infty$ ), depends on the over-compressions $\phi_{i}^{\max }$. Very little over-compression, $\phi_{i}^{\max } \gtrsim \phi_{S J}$, does not change/increase $\phi_{J}$ with cycles $M$, and thus provides a lower limit, $\phi_{S J}$, of the jamming point range. Thus, the isotropic jamming point $\phi_{J}$ is not a unique point, not even for frictionless particle systems, and is dependent on the previous deformation history of the system [37, 149], e.g. over-compression or tapping/driving (data not shown). Both (isotropic) modes of deformation lead to more compact, better packed configurations [21, 167, 220]. Considering different system sizes, and different preparation procedures, we confirmed that the jamming regime is the same (within fluctuations) for all the cases considered, see Appendix Fig. 5.7. All our data are consistent with an important conclusion: Smooth/slow isotropic deformation from random, dilute, unjammed states leads to jamming at a lower-limit density $\phi_{S J}$. Unfortunately this limit is not well defined, i.e. it is highly protocol dependent, not well reproducible, and thus hard to determine experimentally and numerically as well. Reason is that any slow deformation (e.g. compression from below jamming) also leads to perturbations (like tapping leads to granular temperature): the stronger the system is perturbed, the better it will pack, so that usually $\phi_{J}>\phi_{S J}$ is established. Repeated perturbations, see Appendix Fig. 5.6a, lead to a slow stretched exponential approach to an upper-limit jamming density $\phi_{J} \rightarrow \phi_{J}^{\text {max }}$ that itself increases slowly with perturbation amplitude, see Appendix Fig. 5.6b. The observation of different $\phi_{J}$ of a single material, was referred to as $J$-segment $[37,149]$, and only requires an alternative interpretation of the classical "jamming diagram" [21, 116] and to give-up the misconception of a single, constant jamming "point". The state variable $\phi_{J}$ varies due to deformation, but possibly has a lower limit that we denote for now as $\phi_{S J}$. Jammed states below $\phi_{S J}$ might be possible, but require different protocols [81], or different materials, and are thus not addressed here. The concept of shear jammed states [21] below $\phi_{J}$, as illustrated in Fig. 5.1b, is discussed next.

\subsection{Shear jamming below $\phi_{J}(H)$}

To study shear-jamming, we choose several unjammed states with volume fractions $\phi$ below their jamming points ${ }^{1} \phi_{J, i}$, which were established after the first compression-decompression cycle, for different history, i.e., various previously applied over-compression to $\phi_{i}^{\max }$ (Appendix Fig. 5.8). Each configuration is first relaxed and then subjected to four isochoric (volume conserving) pure shear cycles (see Methods). We confirm shear jamming, e.g., by a 
transition in the coordination number $C^{*}$, from below to above its isostatic limit, $C_{0}^{*}=6$, for frictionless grains [84, 158, 185, 209]. This was consistently (independently) reconfirmed by using percolation analysis [21, 162], allowing us to distinguish the three different regimes namely, unjammed, fragile and shear jammed states during (and after) shear, as shown in Appendix Fig. 5.10a. For this, we study the $k$-cluster, defined as the largest network of strong forces, $f \geq k\langle f\rangle[80,184]$. When the initially unjammed isotropic system is sheared, it is growing and percolating first in the compressive direction, then in the neutral, non-mobile direction, and last in the extension direction. The best criterion for identifying growing clusters, the largest of which will percolate in all the three directions, is $k=2.2$, different from $k=1$ for 2D frictional systems [21]. During shear deformation, the fraction of non-rattlers, $f_{\mathrm{NR}}$, increases from initially zero to large values, still well below unity, due to the always existing rattlers. For $f_{\mathrm{NR}}>0.82 \pm 0.01$, we observe that the growing force network is percolated in all three directions (Appendix Fig. 5.10), which is astonishingly similar to the value reported for the 2D systems [21]. From this perspective, when an unjammed material is sheared at constant volume, and it jams after application of sufficient shear strain, clearly showing that the jamming point has moved to a lower value. Shearing the system also perturbs it, just like over-compression; however, in addition, finite shear strains enforce shapeand structure-changes and thus allow the system to explore new configurations; typically, the elevated jamming density $\phi_{J}$ of a previously compacted system will rapidly decrease and exponentially approach its lower-limit, the shear jamming "point" $\phi_{S J}$, below which no shear-jamming exists. These quantification of history dependent jamming densities $\phi_{J}(H)$, due to shear complementing the slow changes by cyclic isotropic (over)compression in Eq. (5.1), is discussed next.

\subsection{Jamming phase diagram with history $H$}

We propose a jamming phase diagram with shear strain, and present a new, quantitative history dependent model that explains jamming and shear-jamming, but also predicts that shearjamming vanishes under some conditions, namely when the system is not tapped, tempered or over-compressed before shear is applied. Using $\varepsilon_{d}$ and $\phi$ as parameters, Fig. 5.2a shows that for one initial the history dependent jamming state at ${ }^{1} \phi_{J, i}$, there exist sheared states within the range $\phi_{S J} \leq \phi \leq \phi_{J}(H)$, which are isotropically unjammed. After small shear strain they become fragile, and for larger shear strain jam and remain jammed, i.e., eventually showing the critical state flow regime, where pressure, shear stress ratio and structural anisotropy have reached their saturation levels and forgotten their initial state (Appendix Fig. 5.9). The transition to fragile states is accompanied by partial percolation of the strong force network, while percolation in all directions indicates the shear-jamming transition. Above jamming, the large fraction of non-rattlers provides a persistent mechanical stability to the structure, even after shear is stopped.

For $\phi$ approaching $\phi_{S J}$, the required shear strain to jam $\varepsilon_{d}^{S J}$ increases, i.e., there exists a 


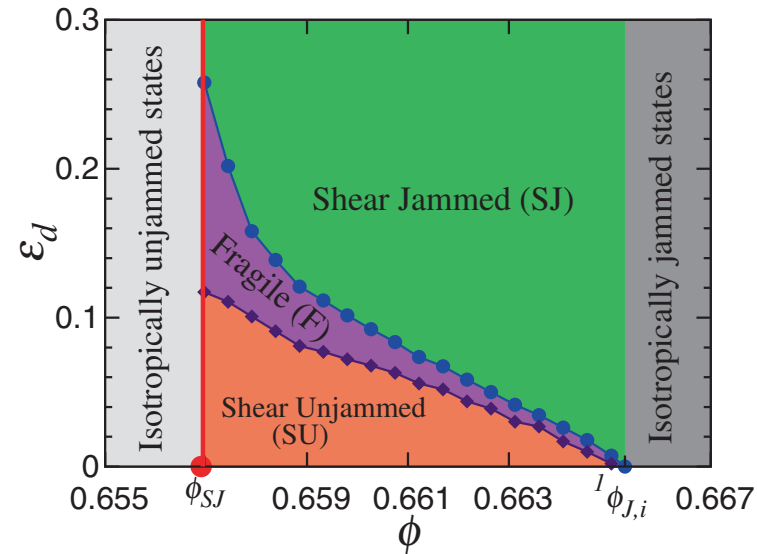

(a)

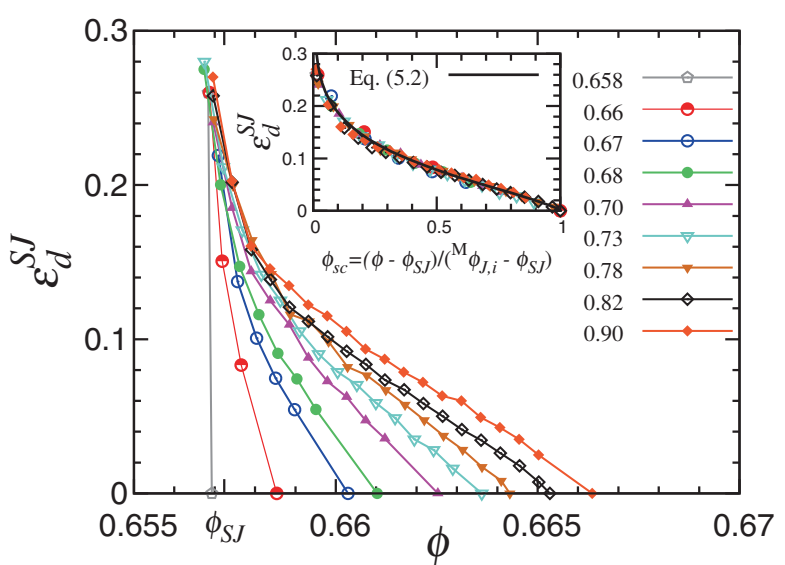

(b)

Figure 5.2: Phase diagram and scaling with $\phi_{S J}$ to replace the ${ }^{M} \phi_{J, i}$ 's. (a) Phase diagram showing the different states: unjammed, isotropic jammed, shear unjammed, fragile and shear jammed, for one particular case of $\phi_{J}\left(\phi_{i}^{\max }=0.82, M=1\right)=:{ }^{1} \phi_{J, i}=0.6652$. (b) Plot of minimum strain needed to jam states prepared from the first over-compression cycle with different $\phi_{i}^{\max }$, as given in the legend. Inset shows collapse of the states using a scaled definition that includes distance from both isotropic jamming point ${ }^{M} \phi_{J, i}$ and shear-jamming point $\phi_{S J}$, using Eq. (5.2). We only show data for the states for $\phi<{ }^{1} \phi_{J, i}$ that after the first isotropic compression decompression cycle jam by applying shear.

divergence "point" $\phi_{S J}$, where 'infinite' shear strain might jam the system, but below which no shear jamming was observed. The closer the (constant) volume fraction $\phi$ is to the initial ${ }^{1} \phi_{J, i}$, the smaller is $\varepsilon_{d}^{S J}$. States with $\phi \geq{ }^{1} \phi_{J, i}$ are isotropically jammed already before shear is applied.

Based on the study of many systems, prepared via isotropic over-compression to a wide range of volume fractions $\phi_{i}^{\max } \geq \phi_{S J}$, and subsequent shear deformation, Fig. 5.2b shows the strains required to jam these states by applying pure shear. A striking observation is that independent of the isotropic jamming point ${ }^{1} \phi_{J, i}$, all curves approach a unique shear jamming point at $\phi_{S J} \sim 0.6567$ (Appendix 5.G). When all the curves are scaled with their original isotropic jamming point ${ }^{M} \phi_{J, i}$ as $\phi_{S C}=\left(\phi-\phi_{S J}\right) /\left({ }^{M} \phi_{J, i}-\phi_{S J}\right)$ they collapse on a unique master curve

$$
\left(\varepsilon_{d}^{S J} / \varepsilon_{d}^{0}\right)^{\alpha}=-\log \phi_{s c}=-\log \left(\frac{\phi-\phi_{S J}}{M_{\phi_{J, i}}-\phi_{S J}}\right)
$$

shown in the inset of Fig. 5.2b, with power $\alpha=1.37 \pm 0.01$ and shear strain scale $\varepsilon_{d}^{0}=$ $0.102 \pm 0.001$ as the fit parameters. Hence, if the initial jamming point ${ }^{M} \phi_{J, i}$ or $\phi_{J}(H)$ is known based on the past history of the sample, the shear-jamming strain $\varepsilon_{d}^{S J}$ can be predicted.

From the measured shear-jamming strain, Eq. (5.2), knowing the initial and the limit value 
of $\phi_{J}$, we now postulate its evolution under isochoric pure shear strain:

$$
\phi_{J}\left(\varepsilon_{d}\right)=\phi_{S J}+\left(\phi-\phi_{S J}\right) \exp \left[\left(\frac{\left(\varepsilon_{d}^{S J}\right)^{\alpha}-\left(\varepsilon_{d}\right)^{\alpha}}{\left(\varepsilon_{d}^{0}\right)^{\alpha}}\right)\right] \text {. }
$$

Inserting, $\varepsilon_{d}=0, \varepsilon_{d}=\varepsilon_{d}^{S J}$ and $\varepsilon_{d}=\infty$ leads to $\phi_{J}={ }^{M} \phi_{J, i}, \phi_{J}=\phi$ and $\phi_{J}=\phi_{S J}$, respectively. This means the jamming point evolution due to shear strain $\varepsilon_{d}$ is faster than exponential (since $\alpha>1$ ) decreasing to its lower limit $\phi_{S J}$. This is qualitatively different from the stretched exponential (slow) relaxation dynamics that leads to the increase of $\phi_{J}$ due to over-compression or tapping, see Fig. 5.3a for both cases.

\subsection{Slow dynamics model}

The last challenge is to unify the observations in a model that accounts for the changes in the jamming densities for both isotropic and shear deformation modes. Over-compressing a soft granular assembly is analogous to tapping $[167,220]$ more rigid ones, in so far that both methods lead to more compact packing structures, i.e., both represent isotropic perturbations. These changes are shown in Fig. 5.1a, where the originally reported logarithmically slow dynamics for tapping [95] is very similar to our results that are also very slow, with a stretched exponential behavior; such slow relaxation dynamics can be explained by a simple Sinai-Diffusion model of random walkers in a random, hierarchical, fractal-type free energy landscape $[127,165]$ in the a-thermal limit, where the landscape does not change - for the sake of simplicity. The granular packing is represented in this picture by an ensemble of random walkers in (arbitrary) configuration space with (potential) energy according to the height of their position on the landscape. (Their average energy corresponds to the jamming density and a decrease in energy corresponds to an increase in $\phi_{J}(H)$, thus representing the "memory" and history dependence.) Perturbations, such as tapping with some amplitude (corresponding to "temperature") allow the ensemble to find denser configurations, i.e., deeper valleys in the landscape, representing larger (jamming) densities. Similarly, overcompression is squeezing the ensemble "down-hill", also leading to an increase of $\phi_{J}$, as presented in Fig. 5.3b. Larger amplitudes will allow the ensemble to overcome larger barriers and thus find even deeper valleys. Repetitions have a smaller chance to do so, which explains the slow dynamics in the hierarchical multi-scale structure of the energy landscape.

In contrast to the isotropic perturbations, where the random walkers follow the "down-hill" trend, shear is anisotropic and thus pushing parts of the system "up-hill". For example, under planar simple shear, one (eigen) direction is tensile (up) whereas an other is compressive (down). If the ensemble is random, shear will only re-shuffle the population. But if the material was previously forced or relaxed towards the (local) land-scape minima, shear can only lead to a net up-hill drift of the ensemble, i.e., to decreasing $\phi_{J}$, referred to as dilatancy. For ongoing perturbation, if volume is conserved, both coordination number and pressure 


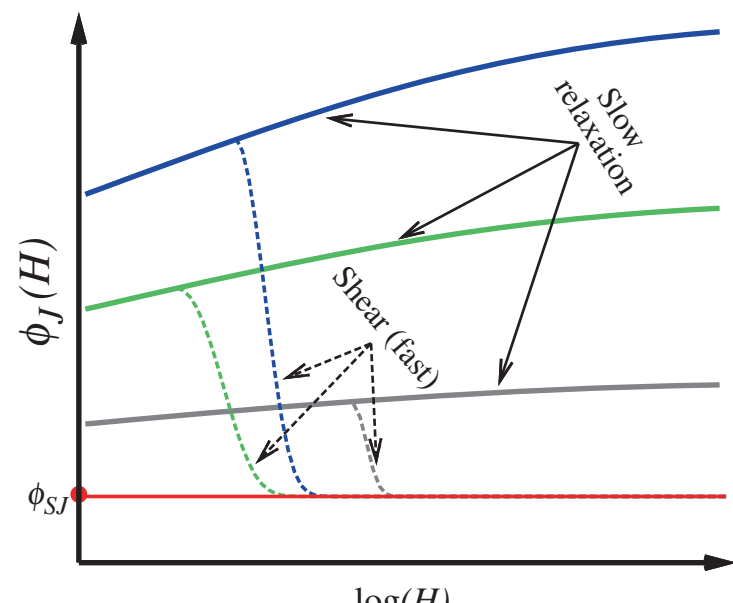

$\log (H)$

(a)

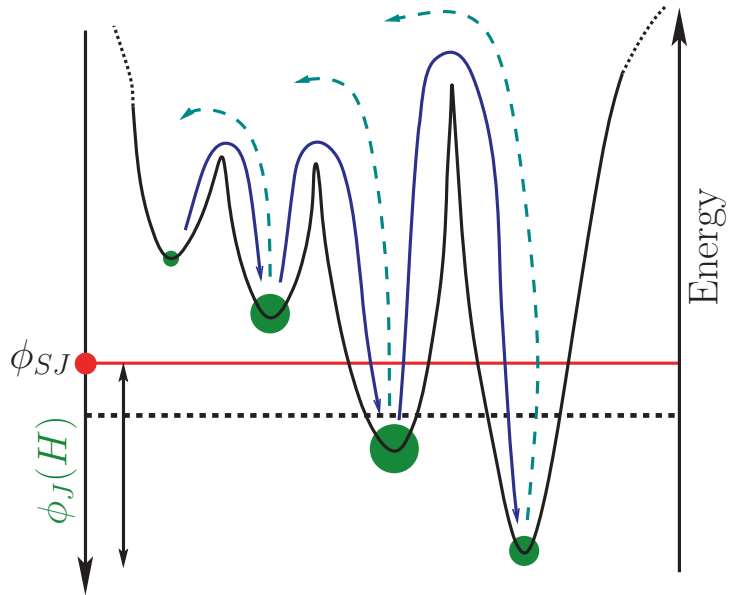

(b)

Figure 5.3: Relaxation dynamics and energy landscape due to memory effects. (a) Evolution of the jamming points $\phi_{J}(H)$ due to history $H$. Solid lines represent many isotropic compression decompression cycles for three different $\phi_{i}^{\max }$, leading to an increase in $\phi_{J}(H)$ by slow stretched exponential relaxation. Dashed lines represent the much faster decrease in $\phi_{J}(H)$ due to shear strain $\varepsilon_{d}$, using Eq. (5.3). (b) The sketch represents only a very small, exemplary part of the hierarchical, fractal-type landscape. The red horizontal line represents the (quenched) average, while the dotted horizontal line indicates the momentary average $\phi_{J}(H)$. The blue solid arrows show relaxation due to perturbations, while the dashed arrows indicate re-arrangements (re-juvenation) due to large shear strain. The green dots represent with their size the population after some relaxation, in contrast to a random, quenched population where all equal size valleys are equally populated [214].

slowly decrease (Appendix Fig. 5.9) whereas for fixed confining pressure (data not shown) the volume would decrease (compactancy). This process is much faster than relaxation, since it is driven by shear strain amplitude. For large enough strain the system will be sufficiently re-shuffled, randomized, or "re-juvenated" such that it can be close to its quenched, random state $\phi_{S J}$.

\subsection{Prediction: minimal model}

Finally, we test the proposed history dependent jamming point $\phi_{J}(H)$ model, by predicting $p$ and $C^{*}$, when a granular assembly is subjected to cyclic isotropic compression to $\phi_{i}^{\max }=0.73$ for $M=1$ and for $M=300$ cycles, with ${ }^{\infty} \phi_{J, i}=0.667$, as shown in Fig. 5.4(a-b) (prediction details discussed in Appendix 5.H). It is observed that using the history dependence of $\phi_{J}(H)$, the hysteretic behavior of the isotropic quantities, $p$ and $C^{*}$, is very well predicted, qualitatively similar to isotropic compression and decompression of real $2 \mathrm{D}$ frictional granular assemblies, as shown in Fig. 7 by Bandi et al. [12]. 


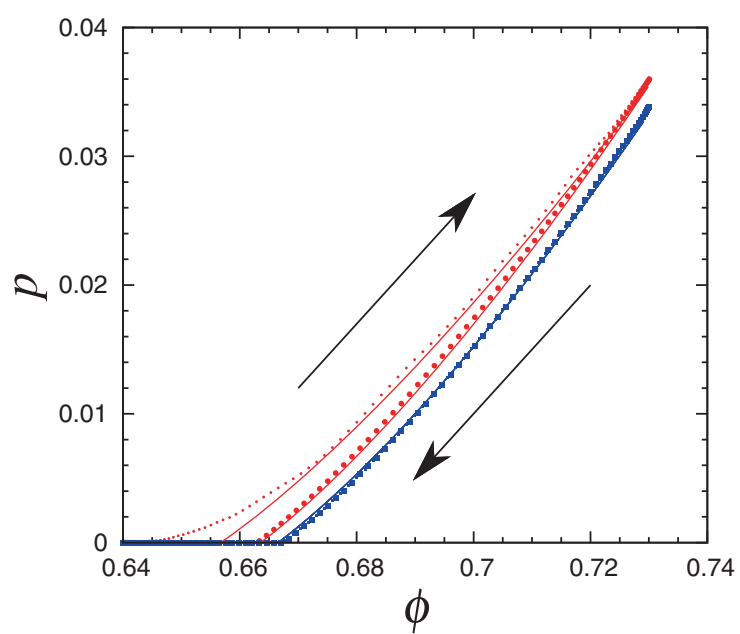

(a)

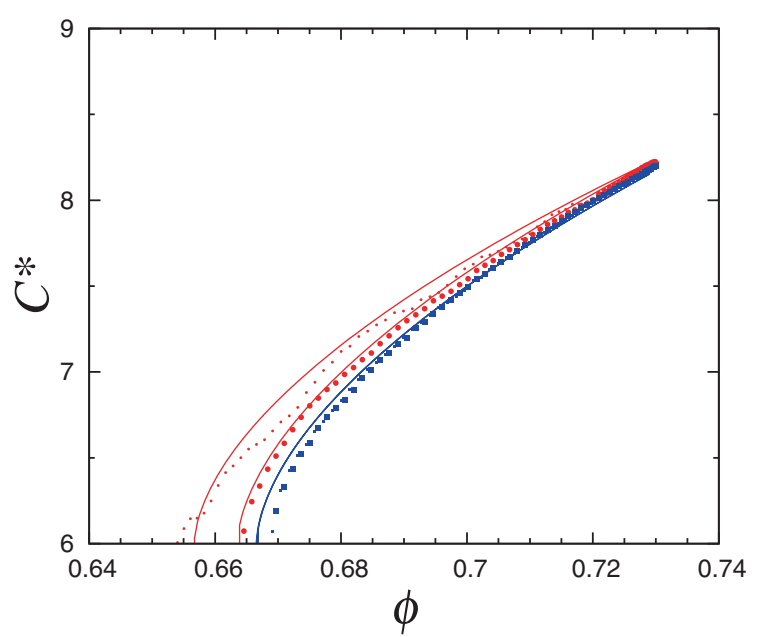

(b)

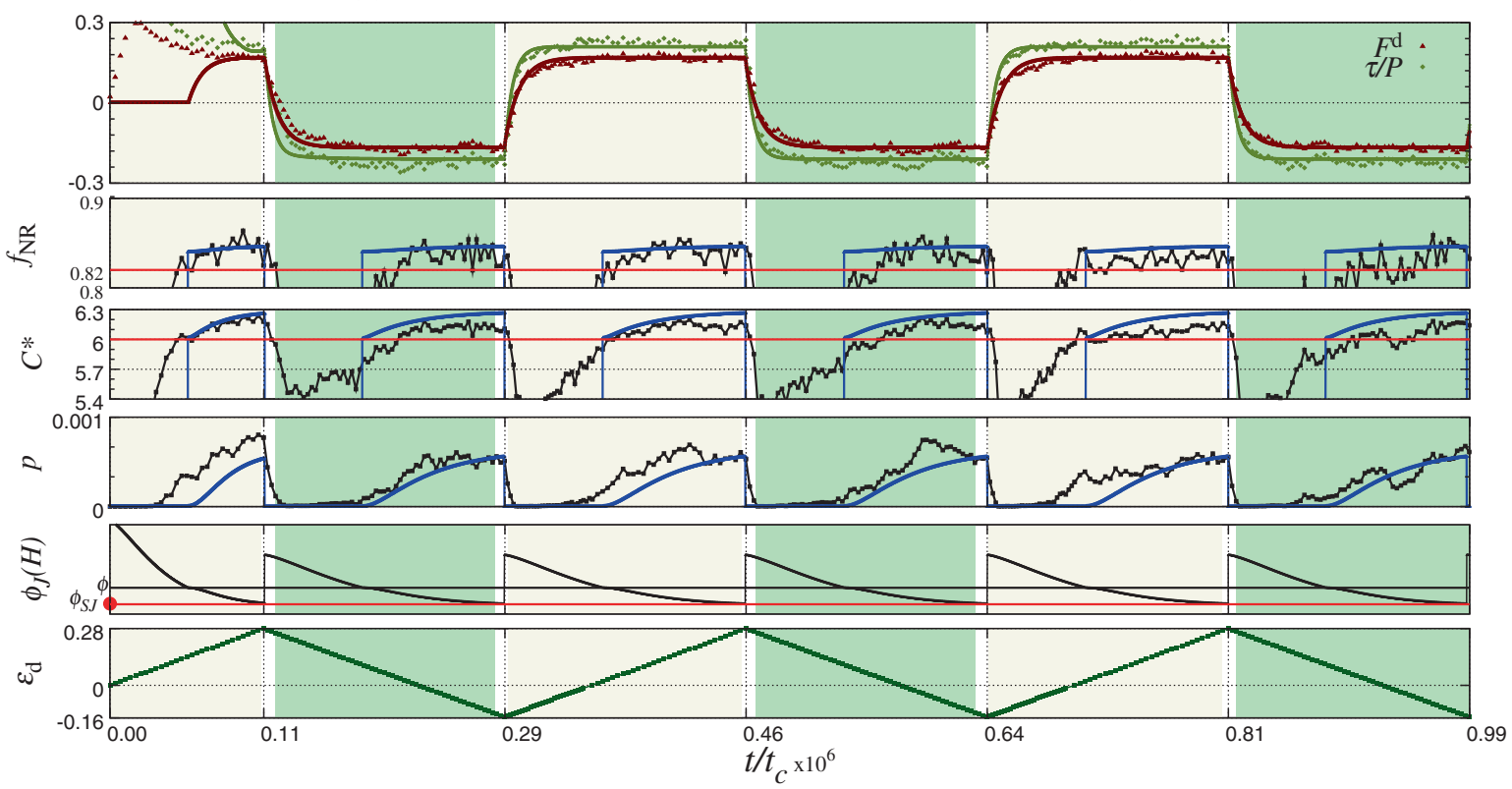

(c)

Figure 5.4: Model prediction of cyclic isotropic over-compression and cyclic shear: (a) Dimensionless pressure $p$ and (b) coordination number $C^{*}$ plotted against volume fraction $\phi$ for an isotropic compression starting from $\phi_{t}=0.64$ to $\phi_{i}^{\max }=0.73$ (small symbols) and decompression (big symbols) back to $\phi_{t}$, with ${ }^{\infty} \phi_{J, i}=0.667$, for $M=1$ (red ' $\bullet$ ') and for $M=300$ (blue ' $\boldsymbol{\square}$ '). Deviatoric stress ratio $\tau / p$ and deviatoric fabric $F^{\mathrm{d}}$, fraction of nonrattlers $f_{\mathrm{NR}}$, coordination number $C^{*}$, pressure $p$ and history dependent jamming point $\phi_{J}(H)$ over three pure shear strain cycles (bottom panel) for $\phi=0.6584$ and initial jamming point $\phi_{J}\left(\phi_{i}^{\max }=0.82, M=1\right)=:{ }^{1} \phi_{J, i}=0.6652$. Solid lines through the data are the model prediction, involving the history dependent jamming point $\phi_{J}(H)$, using Eq. (5.1) for isotropic deformation and Eq. (5.3) for shear deformation, and others, as described in Appendix 5.H. Horizontal red lines in $f_{\mathrm{NR}}$ and $C^{*}$ represent transition from unjammed to shear jammed states, whereas in $\phi_{J}(H)$ indicates the shear jamming point $\phi_{S J}$. 
In Fig. 5.4c, we show the evolution of the deviatoric quantities shear stress ratio $\tau / p$ and deviatoric fabric $F^{\mathrm{d}}$, when a system with $\phi=0.6584$, close to $\phi_{S J}$, and initial jamming point $\phi_{J}(0)=0.6652$, is subjected to three shear cycles (lowest panel). The shear stress ratio $\tau / p$ is initially undefined, but soon establishes a maximum (not shown) and decays to its saturation level at large strain. After strain reversal, $\tau / p$ drops suddenly and attains the same saturation value, for each half-cycle, only with alternating sign. The behavior of the anisotropic fabric $F^{\mathrm{d}}$ is similar to that of $\tau / p$. During the first loading cycle, the system is unjammed for some strain, and hence $F^{\mathrm{d}}$ is zero in the model (observations in simulations can be non-zero, when the data correspond to only few contacts, mostly coming from rattlers). However, the growth/decay rate and the saturation values attained are different from those of $\tau / p$, implying a different, independent stress- and structure-evolution with strain - which is at the basis of recently proposed anisotropic constitutive models for quasi-static granular flow under various deformation modes [84]. The simple model with $\phi_{J}(H)$, is able to predict quantitatively the behavior the $\tau / p$ and $F^{\mathrm{d}}$ after the first loading path, and is qualitatively close to the cyclic shear behavior of real 2D frictional granular assemblies, as shown in Supplementary Fig. 7 by Bi et al. [21].

At the same time, also the isotropic quantities are very well predicted by the model, using the simple equations from Appendix 5.H, where only the jamming point is varying with shear strain, while all material parameters are kept constant. Some arbitrariness involves the sudden changes of $\phi_{J}$ at reversal, as discussed in Appendix 5.H. Therefore, using a history dependent $\phi_{J}(H)$ gives hope to understand the hysteretic observations from realistic granular assemblies, and also provides a simple explanation of shear-jamming. Modifications of continuum models like anisotropic models [84], or GSH type models [89], by including a variable $\phi_{J}$, can this way explain also transitions around jamming.

\subsection{Interpretation and Outlook}

In summary, the questions posed in the introduction can now be answered: $(i)$ Shearjamming occurs in 3D without any friction; (ii) the transition between the jammed and flowing (unjammed) regimes is controlled by a single, isotropic, history dependent state variable, the jamming density $\phi_{J}(H)$, which (iii) has an absolute minimum jamming density; so that $(i v)$ the protocol dependence of jamming is completely explained by the new state variable.

Given an extremely simple model picture, starting from an isotropically unjammed point, shear-jamming is not anymore a new effect, but is just due to the shift of the state variable jamming density to lower values during shear. (The evolution equations with their material parameters are determined quantitatively from a set of different simulations). Shear jamming occurs when the variable $\phi_{J}(H)$ crosses the (fixed) $\phi$ of the system. The model implies now a minimum $\phi_{J} \geq \phi_{S J}$ that represents the critical (steady) state in the limit of vanishing confining 
stress, i.e., the lower limit of all jamming points. This is nothing else than the lowest stable random density a sheared system locally can reach due to continuously ongoing shear, for vanishing confining stress.

This lower limit might be difficult to access in experiments and simulations, since every shear also perturbs the system leading at the same time to (slow) relaxation and thus a competing increase in $\phi_{J}(H)$. However, it can be obtained from the relaxed, critical state values of pressure, extrapolated to zero, i.e., from the envelope in Appendix Fig. 5.12. The history dependent jamming point $\phi_{J}(H)$ is difficult to access directly, but can consistently be extracted from experimentally measurable quantities, e.g. pressure $p$, coordination number $C^{*}$ or fraction of non-rattlers $f_{\mathrm{NR}}$. We explain the methodology to extract $\phi_{J}(H)$ experimentally, and confirm by indirect measurement, as details in Appendix 5.H - 5.I that the jamming density is indeed increasing during isotropic deformation and decreasing during shear.

Experiments should be performed to calibrate our model given real materials. Over-compression is possible for soft materials, but not expected to lead to considerable relaxation due to the small possible compressive strain for harder materials. However, tapping or small-amplitude shear can take the role of over-compression, also leading to perturbations and increasing $\phi_{J}$; in contrast, large-amplitude shear is decreasing $\phi_{J}$ and can be calibrated indirectly from different isotropic quantities. The accessible range of $\phi_{J}-\phi_{S J}$ is expected to much increase for more realistic systems, e.g., with friction, or non-spherical particle shapes. A measurement of the landscape, e.g. the valley width, depth and shapes [214] should be done to verify our model-picture, as this remains qualitative so far.

\subsection{Methods}

Discrete particle simulations are used to model the deformation behavior of systems with $N=9261$ soft frictionless spherical particles with average radius $\langle r\rangle=1[\mathrm{~mm}]$, density $\rho=2000\left[\mathrm{~kg} / \mathrm{m}^{3}\right]$, and a uniform polydispersity width $w=r_{\max } / r_{\min }=3$, using the linear visco-elastic contact model in a 3D box with periodic boundaries [84]. The particle stiffness is $k=10^{8}\left[\mathrm{~kg} / \mathrm{s}^{2}\right]$, contact viscosity is $\gamma=1[\mathrm{~kg} / \mathrm{s}]$, and the smallest contact duration is $t_{c}=0.2279[\mu \mathrm{s}][84]$. Firstly, the particles are generated with random velocities at $\phi=$ 0.3 and are isotropically compressed to $\phi_{t}=0.64<\phi_{S J}$, and later relaxed. The system is further isotropically compressed to different $\phi_{i}^{\max }$ and decompressed back to $\phi_{t}$, and is repeated over $M$ cycles, which provides the ${ }^{M} \phi_{J, i}$ (Appendix 5.B). Later, several isotropic configurations $\phi$, such that $\phi_{t}<\phi<{ }^{1} \phi_{J, i}$ from the decompression branch are chosen as the initial configurations. We relax them and apply pure (volume conserving) shear with the strain-rate tensor $\dot{\mathbf{E}}= \pm \dot{\varepsilon}_{\mathrm{dev}}(-1,1,0)$, for four cycles (Appendix Figs. 5.8 and 5.9). The $x$ and $y$ walls move, while the $z$ wall is stationary. Note that the strain rate of the (quasi-static) deformation is small, $\dot{\varepsilon}_{\mathrm{dev}} t_{c}<3.10^{-6}$, to avoid transient behavior. 
Pressure $P=\operatorname{tr}(\boldsymbol{\sigma}) / 3$, where $\boldsymbol{\sigma}$ is the stress tensor [21, 84]. The fabric tensor is defined as $\mathbf{F}=(1 / V) \sum_{\mathcal{P} \in V} V^{\mathcal{P}} \sum_{c \in \mathcal{P}} \mathbf{n}^{c} \otimes \mathbf{n}^{c}$, where $V^{\mathcal{P}}$ is the particle volume for all particles $\mathcal{P}$ lying in $V$, and $\mathbf{n}^{c}$ is the normal unit branch-vector pointing from center of particle $\mathcal{P}$ to contact $c$. Isotropic part of fabric is $F_{\mathrm{V}}=\operatorname{tr}(\mathbf{F})$. The corrected coordination number $[21,84]$ is $C^{*}=M_{4} / N_{4}$, where, $M_{4}$ is total contacts of the $N_{4}$ particles having at least 4 contacts, and the non-rattler fraction is $f_{\mathrm{NR}}=N_{4} / N$. For any tensor $\mathbf{Q}$, its deviatoric part is, $Q_{\mathrm{dev}}=\operatorname{sgn}\left(q_{y y}-q_{x x}\right) \sqrt{3 q_{i j} q_{i j} / 2}$, where $q_{i j}$ are the components of the deviator of $\mathbf{Q}$, and the sign function accounts for the shear direction. Both pressure $P$ and shear stress $\Gamma$ are non-dimensionalized by $2\langle r\rangle / k$ to give dimensionless pressure $p$ and shear stress $\tau$. The shear-jamming point is obtained using the relaxed, critical state data sets of $p$, as presented in Appendix 5.G.

\section{A Data analysis}

In this appendix, we present in detail the methodology and results from elaborate data analysis used to understand the slow relaxation/compaction experiments and their structural origin, and the relation between jamming and shear-jamming in three-dimensions for frictionless, polydisperse spherical particles in a periodic box. The report is organized as follows: In section 5.B, we present a procedure to identify the jamming-points and their range. In section 5.C, we show the effect of cyclic over-compression to different target volume fractions and present a model that captures this phenomena. Section 5.D is devoted to show a statistical analysis and sensitivity of the jamming point with the system size (number of particles) and different initial configurations. Section 5.E is devoted to the effect of cyclic pure shear experiments and their effect on the microscopic and macroscopic quantities. In section 5.F, we discuss the results on growing, percolating clusters during cyclic shear with focus on reversal and their link with the non-rattler fraction. In section 5.G, we explore the phase space of macroscopic quantities during shear and the effects of relaxation, compaction and creep in critical (steady) state, after large strain amplitude, when the initial state is forgotten. There, we also present the methodology used to obtain the shear jamming point. In section 5.H, we show the analytical model, especially the history dependent jamming point, that is used for the prediction of macroscopic quantities. Finally in section 5.I, we present a procedure to extract the history dependent jamming point from experimentally measurable quantities.

\section{B Identification of the jamming point}

In the of this paper, we have presented ranges of the isotropic jamming points, where pressure vanishes, that are possible due to different sample histories. Before we explain the procedure for identification of jamming points, we want to point out here that the stress tensor $\sigma$, that is used to define the dimensionless pressure $p$ and shear stress $\tau$, has static and dynamic 
contributions, with the latter being four orders of magnitude smaller than the former (in the cases studied) and hence the dynamic contributions are neglected.

When a sample is over-compressed isotropically, the loading and unloading paths are different in pressure $p$. This difference is most pronounced near the jamming point $\phi_{J}$, and for the first cycle. It brings up the first question of how to identify a jamming point, $\phi_{J}$. The unloading branch of a cyclic isotropic over-compression along volume fraction $\phi$ is well described by a linear relation in volumetric strain, with a quadratic correction:

$$
p=\frac{\phi C}{\phi_{J}} p_{0}\left(-\varepsilon_{\mathrm{v}}\right)\left[1-\gamma_{p}\left(-\varepsilon_{\mathrm{v}}\right)\right]
$$

where $p_{0}, \gamma_{p}$ presented in Table 5.1, and the jamming point $\phi_{J}$ are the fit parameters. $-\varepsilon_{\mathrm{v}}=$ $\log \left(\phi / \phi_{J}\right)$ is the true or logarithmic volumetric strain of the system, defined relative to the reference where $p \rightarrow 0$, i.e. jamming volume fraction. $C$ is the ratio of total non-rattler contacts $M_{4}$ and total number of particles $N$, i.e., $C=M_{4} / N=\left(M_{4} / N_{4}\right)\left(N_{4} / N\right)=C^{*} f_{\mathrm{NR}}$, with corrected coordination number $C^{*}$ and fraction of non-rattlers $f_{\mathrm{NR}}$. Using Eq. (5.4), we can extrapolate $p$ to zero, to get $\phi_{J}$. We apply the same procedure for different overcompressions, $\phi_{i}^{\max }$, and many subsequent cycles $M$ to obtain ${ }^{M} \phi_{J, i}$, for which the results are discussed below. The material parameter $p_{0}$ is finite, almost constant, whereas $\gamma_{p}$ is small, sensitive to history and contributes mainly for large $-\varepsilon_{\mathrm{v}}$, with values ranging around $0 \pm 0.1$; in particular, it is dependent on the over-compression $\phi_{i}^{\max }$ (data not shown). Unless strictly mentioned, we shall be using the values of $p_{0}$ and $\gamma_{p}$ given in Table 5.1.

Fig. 5.5a shows the behavior of $p$ with $\phi$ during one full over-compression cycle to display the dependence of the jamming point on the maximum over-compression volume fraction and the number of cycles. With increasing over-compression amplitude, e.g. comparing $\phi_{i}^{\max }=0.68$ and $\phi_{i}^{\max }=0.82$, the jamming point, as realized after unloading, is increasing. Also, with each cycle, from $M=1$ to $M=100$, the jamming point moves to larger values. Note that the difference between the loading and the unloading curves becomes smaller for subsequent over-compressions. Fig. 5.5b shows the scaled pressure, i.e., $p$ is normalized by $\phi C / \phi_{J}$, which removes its non-linear behavior. This scale pressure $p$ represents the average deformation (overlap) of the particles at a given volume fraction, proportional to the distance from the jamming point $\phi_{J}$. In the small strain region, for all over-compression amplitude and cycles, the datasets collapse on a line with slope $p_{0} \sim 0.04$. Only for very strong overcompression, $-\varepsilon_{\mathrm{v}}>0.1$, a small deviation (from linear) of the simulation data is observed due to the tiny quadratic correction in Eq. (5.4).

\section{C Isotropic cyclic over-compression}

In this section, we present a simple model that captures the increasing ${ }^{M} \phi_{J, i}$ behavior with increasing over-compression, $\phi_{i}^{\max }$, as well as cycles, $M$. In Fig. 5.6a (the same data as in 


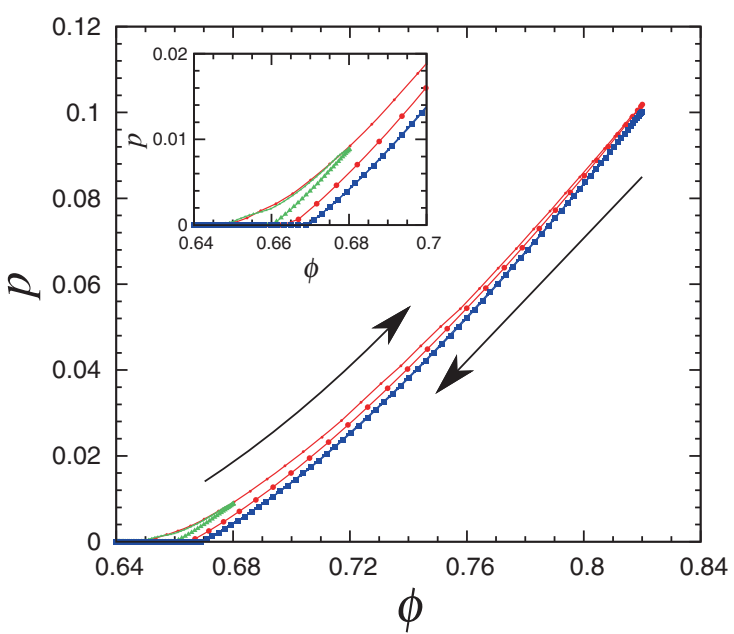

(a)

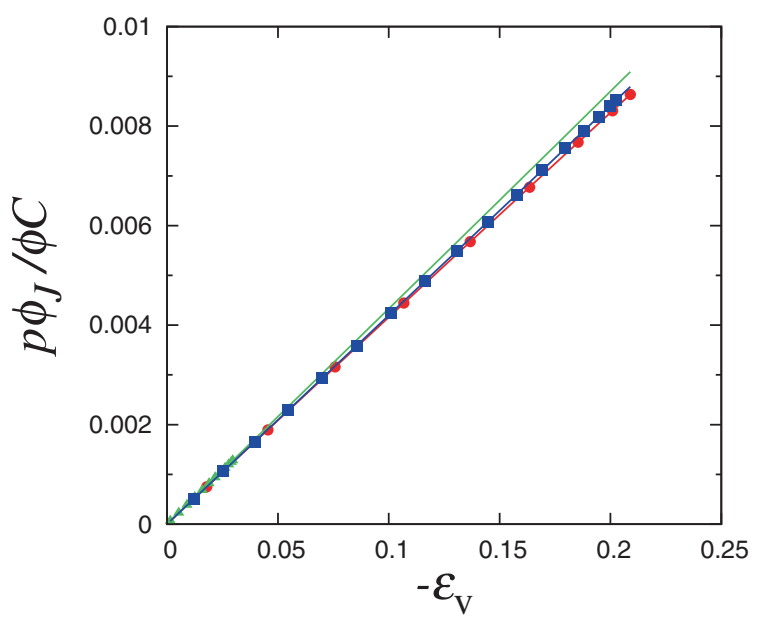

(b)

Figure 5.5: (a) Dimensionless pressure $p$ plotted against volume fraction $\phi$ and for an isotropic compression starting from $\phi_{t}=0.64$ to $\phi_{i}^{\max }=0.68$ (green ' $\boldsymbol{\nabla}$ ') and $\phi_{i}^{\max }=0.82$ (red ' $\bullet$ ') and decompression back to $\phi_{t}$ for $M=1$, leading to ${ }^{1} \phi_{J}\left(\phi_{i}^{\max }=0.68\right)=0.6610$ and ${ }^{1} \phi_{J}\left(\phi_{i}^{\max }=0.82\right)=0.6652$. The blue ' $\backsim$ ' data points represent cyclic over-compression to $\phi_{i}^{\max }=0.82$ for $M=100$, leading to ${ }^{100} \phi_{J}\left(\phi_{i}^{\max }=0.82\right)=0.6692$. The upward arrow indicates the loading path (small symbols) while the downward arrow indicates the unloading path (big symbols). The inset is the zoomed in regime near the jamming point. Lines are connecting the datasets only (b) Scaled pressure $p \phi_{J} / \phi C$ plotted against volumetric strain $-\varepsilon_{\mathrm{v}}=\log \left(\phi / \phi_{J}\right)$ for the same simulations in (a). The $\phi_{J}$ are extracted using a fit to Eq. (5.4). Lines passing through the data represents scaled pressure, when Eq. (5.4) is restructured.

Fig. 5.1a are shown on a logarithmic scale, with fits using Eq. (5.1)), weak over-compression does not lead to a significant increase in $\phi_{J, i}$, giving us information about the lower limits of the isotropic jamming points, which is the shear jamming point $\phi_{S J}=0.6567$, see section 5.G. With each over-compression cycle, ${ }^{M} \phi_{J, i}$ increases, but for large $M$ it increases less and less. This is analogous to compaction by tapping, where the tapped density increases logarithmically slow with the number of taps. Several models are available in literature to depict this density relaxation behavior e.g. hyperbolic tangent, stretched exponential, inverse logarithmic, reciprocal linear laws etc.. We tried these four models and found that they fit our data qualitatively well; however, the data is best predicted by a Kohlrausch-Williams-Watts (KWW) stretched exponential relation (Eq. (5.1)).

It is interesting to note that with increasing $\phi_{i}^{\max }$, the limit value ${ }^{\infty} \phi_{J, i}$ increases and gives an upper bound to the isotropic jamming points that can be achieved, while the lower bound is limited by $\phi_{S J}$, as shown in Fig. 5.6b. This leads to the $J$-segment, i.e., the explorable region of jamming points. The limit value ${ }^{\infty} \phi_{J, i}$ with $\phi_{i}^{\max }$ can be fitted with a simple power law 


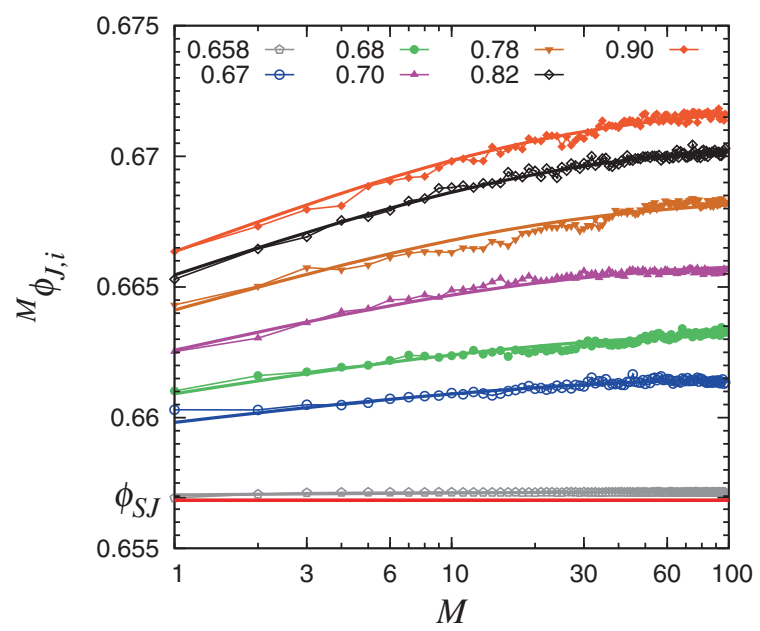

(a)

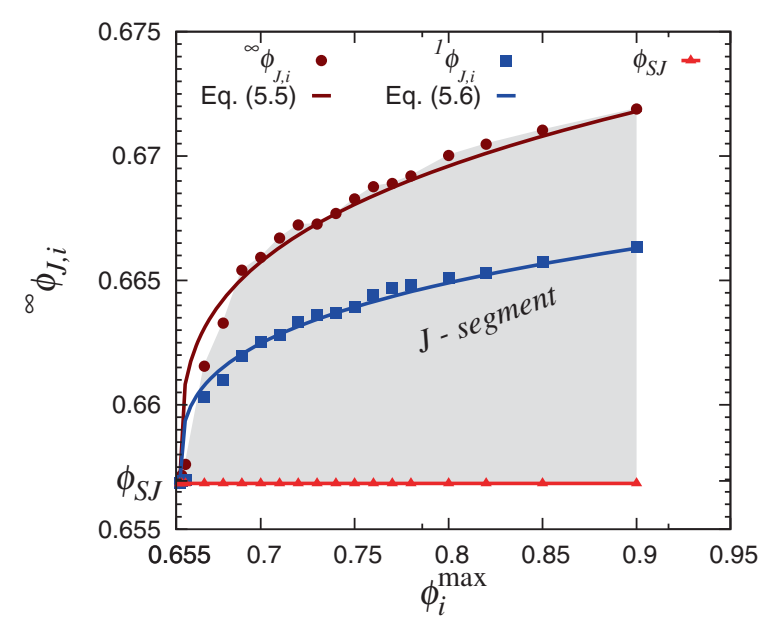

(b)

Figure 5.6: (a) Fig. 5.1a reproduced on a logarithmic $M$-axis, where the solid lines passing through the data are the fits using Eq. (5.1). Note that not all $\phi_{i}^{\max }$ data are plotted for better clarity. (b) The first jamming point ${ }^{1} \phi_{J, i}$ (blue ' $\square$ ') and after many over-compression ${ }^{\infty} \phi_{J, i}$ (brown ' $\bullet$ ') are plotted against over-compression amplitude $\phi_{i}^{\max }$. Solid lines represent Eqs. (5.5) for ${ }^{\infty} \phi_{J, i}$ and (5.6) for ${ }^{1} \phi_{J, i}$. The shaded region is the explorable range of jamming points ${ }^{M} \phi_{J, i}$, denoted as $J$-segment. The red base line indicates the shear jamming point $\phi_{S J}$.

relation:

$$
{ }^{\infty} \phi_{J, i}=\phi_{S J}+\alpha_{\max }\left(\phi_{i}^{\max } / \phi_{S J}-1\right)^{\beta},
$$

where the fit works perfect for $\phi_{S J}<\phi_{i}^{\max } \leq 0.9$, with parameters $\phi_{S J}=0.6567, \alpha_{\max }=$ $0.02 \pm 2 \%$, and $\beta=0.3$, while the few points for $\phi_{i}^{\max } \sim \phi_{S J}$ are not well captured.

The relation between the limit-value ${ }^{\infty} \phi_{J, i}$ and ${ }^{1} \phi_{J, i}$ is derived from Eq. (5.1):

$$
{ }^{\infty} \phi_{J, i}-\phi_{S J}=\frac{{ }^{1} \phi_{J, i}-\phi_{S J}}{1-e^{-1}} \cong 0.632\left({ }^{1} \phi_{J, i}-\phi_{S J}\right)
$$

only by setting $M=1$, as shown in Fig. 5.6b, with perfect match. With other words, using a single over-compression, Eq. (5.6) allows to predict the limit value after first overcompression ${ }^{1} \phi_{J, i}$ (or subsequent over-compression cycles, using appropriate $M$ ).

\section{D Effect of system size and initial configurations}

Next, we study the effect of system size and different initial configurations on the jamming point for same different $\phi_{i}^{\max }$. In Fig. 5.7a, jamming points ${ }^{1} \phi_{J, i}$ are plotted; the samples are prepared with the same procedure as mentioned in section 5.8, but with varying number of particles $N$ in the system. Within the fluctuations, this tells us that the jamming points 


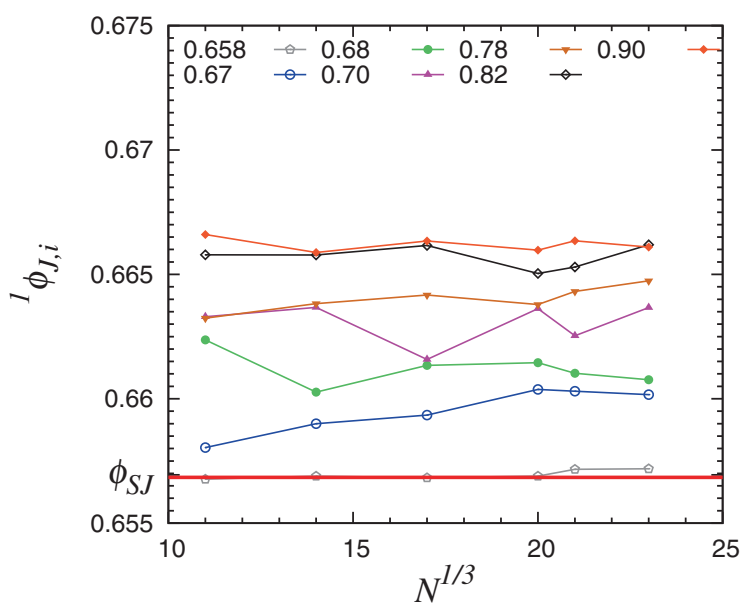

(a)

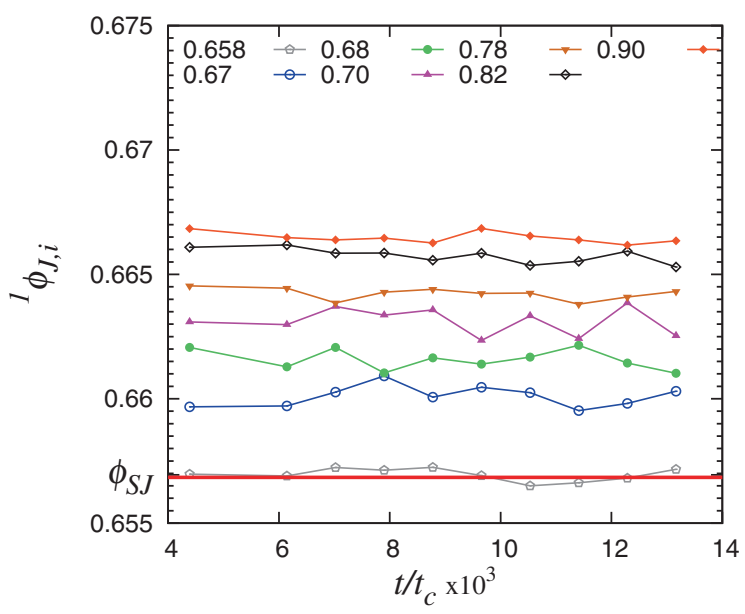

(b)

Figure 5.7: (a) Effect of system size (number of particles $N$ ) on the isotropic jamming point $\phi_{J}$. The preparation procedure is the same for all cases. All the samples were overcompressed from $\phi_{t}=0.64$ to $\phi_{i}^{\max }$ and decompressed back to $\phi_{t}$. (b) Effect of different initial system configurations on the isotropic jamming point $\phi_{J}$, which were prepared by applying time $t$ of compression from $\phi=0.3$ to $\phi_{t}$. The compression time $t$ is scaled with the smallest contact time between two particles $t_{c}$. Later, all the samples were compressed to various $\phi_{i}^{\max }$ and then decompressed back to $\phi_{t}$ with the same rate for the first cycle. The red line indicates the shear jamming limit $\phi_{S J}$.

are independent of the system size. In Fig. 5.7b, we present the effect of different initial configurations on the jamming points ${ }^{1} \phi_{J, i}$, having the same number of particle $N=9261$. The compression times during isotropic compression from a loose state are varied, which provides the possibility of producing different initially unjammed configurations, due to random statistical fluctuations. Later the samples are compressed to $\phi_{i}^{\max }$ and decompressed back to $\phi_{t}$ during one cycle $(M=1)$. We observe only small fluctuations in ${ }^{1} \phi_{J, i}$ with different configurations, and hence independence on the initial configurations, provided that the main cycle-experiments are performed in the quasi-static limit.

\section{E Cyclic Shear results}

Here, we will discuss the effect of cyclic pure shear on some microscopic and macroscopic quantities, namely the corrected coordination number $C^{*}$, the pressure $p$, and stress $\tau$, the shear stress ratio $\tau / p$, and the anisotropic fabric $F^{\mathrm{d}}$ and their evolution for different volume fractions below isotropic jamming point. Firstly, from the observations in section 5.C, it is easy to say that there exist $\phi$ such that $\phi_{S J} \leq \phi \leq \phi_{J}^{\max }$, where it is possible to generate isotropic unjammed states and jammed states corresponding to higher and lower overcompressions respectively, is shown in Fig. 5.8. Secondly, shearing at constant volume can 


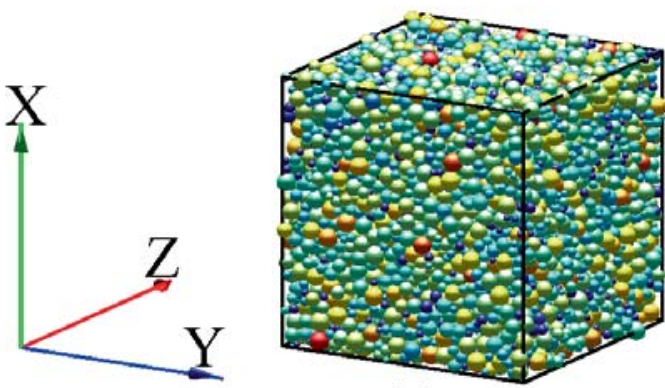

(a)

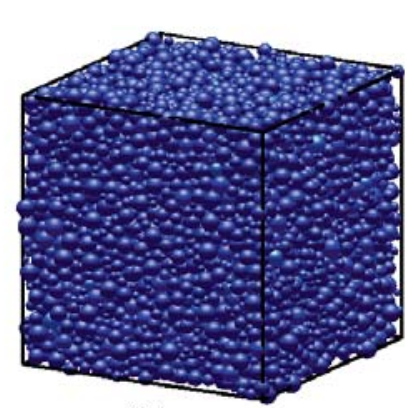

(b)
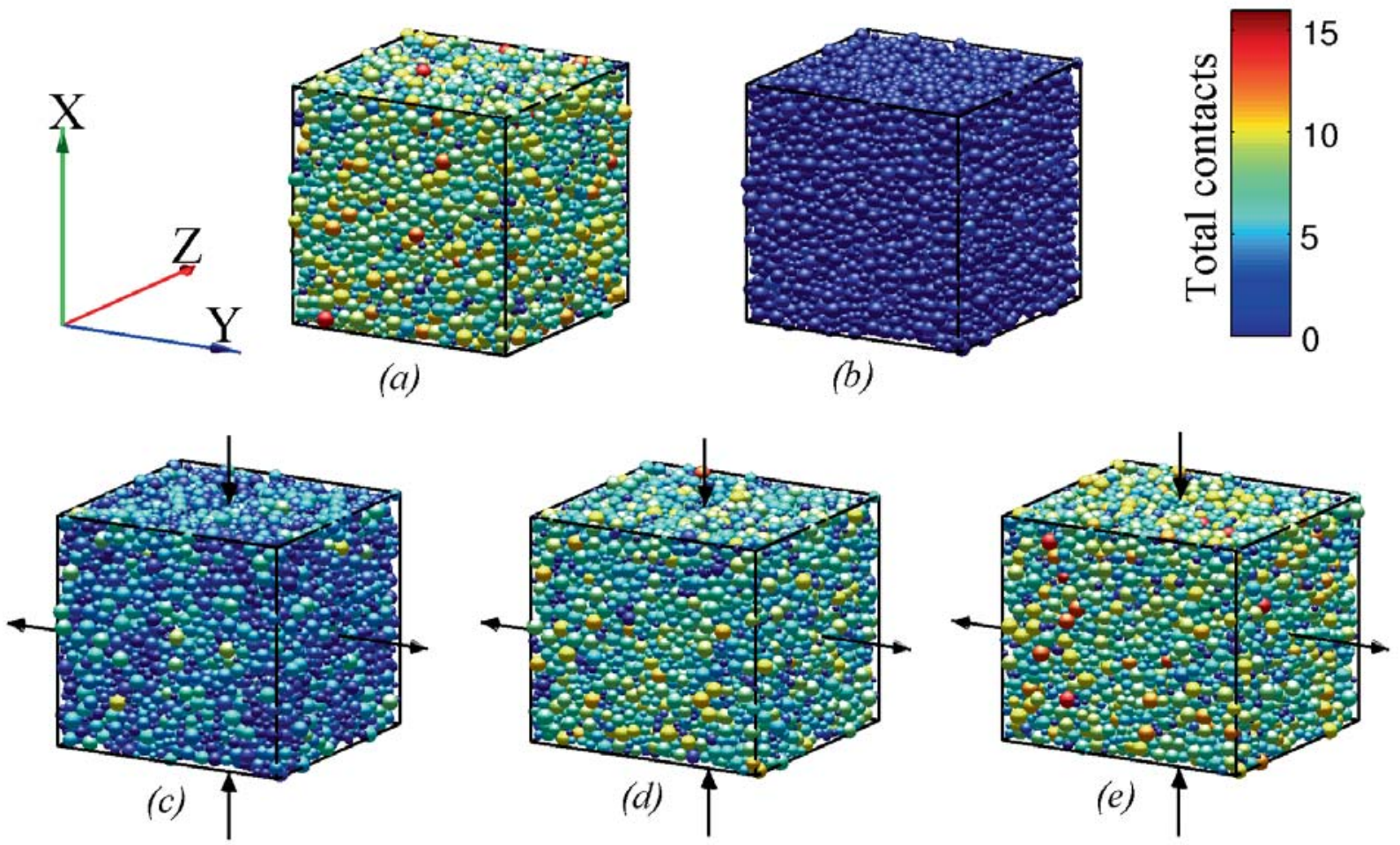

Figure 5.8: Snapshot of the (a) isotropically jammed packing with volume fraction $\phi=$ 0.6584 and $\phi_{J}\left(\phi_{i}^{\max }=0.66, M=1\right)=:{ }^{1} \phi_{J, i}=0.6585$; (b) unjammed packing with the same volume fraction and $\phi_{J}\left(\phi_{i}^{\max }=0.82, M=1\right)=:{ }^{1} \phi_{J, i}=0.6652$, which is then sheared to get : (c) Unjammed packing at strain $\varepsilon_{d}=0.0492$, with some unstable, temporary contacts; (d) Fragile packing at strain $\varepsilon_{d}=0.0985$; (e) Shear jammed packing at strain $\varepsilon_{d}=0.1611$. The colorbar indicates the total number of contacts of a particle.

generate shear unjammed, fragile and finally shear jammed states for different shear strain $\varepsilon_{d}$ values, as seen in Fig. 5.10.

In Fig. 5.9, we plot the evolution of microscopic and macroscopic quantities during volume conserving cyclic shear experiments for $\phi=0.6593$. Since the state is below jamming, $C^{*}$ grows slowly from 0 and crosses the isostatic coordination number $C_{0}^{*}=6$ as shown in Fig. 5.9a. As we apply further shear strain, $C^{*}$ attains an asymptotic value with small fluctuations. At reversal, the particles start to loose contacts due to changes in the main force network direction, hence $C^{*}$ decreases sharply. Afterwards, with increase in opposite shear strain, $C^{*}$ starts to build up again and reaches a similar, fluctuating limit value as for initial strain. For $p$, similar behavior like for $C^{*}$ vs $\varepsilon_{d}$, is seen in Fig. 5.9b, where $p$ grows from 0 (up to finite strain $\varepsilon_{d} \leq 0.08$ ) to higher values until it reaches its asymptotic value, with relatively larger fluctuations. After the initial cycle, both $C^{*}$ and $p$ vs $\varepsilon_{d}$ collapse on top of each other. Note that the plot of $C^{*}$ and $p$ vs $\varepsilon_{d}$ is symmetric, not about the original box configuration but about the mean of the large extremes $\varepsilon_{d}^{\max }$ and $\varepsilon_{d}^{\min }$, i.e., $0.5 *(0.28-0.16)=0.06$, so in a way providing history-independence, and deviatoric limit state symmetry. In Figs. 5.9c and $5.9 \mathrm{~d}$, we plot the variation of $C^{*}$ and $p$ vs $\varepsilon_{d}$ for different volume fractions, respectively, for 


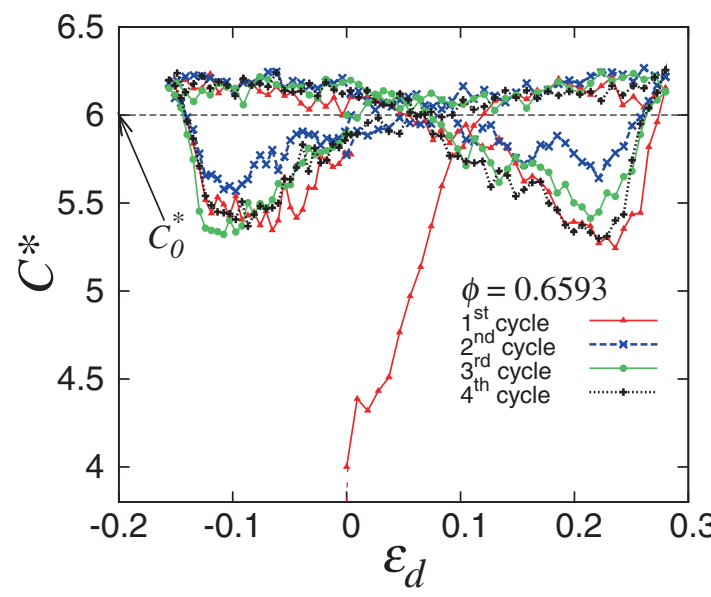

(a)

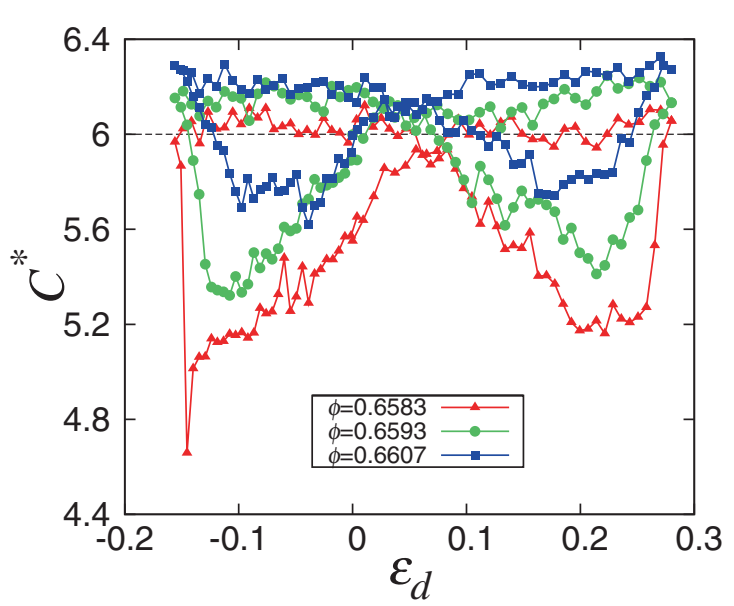

(c)

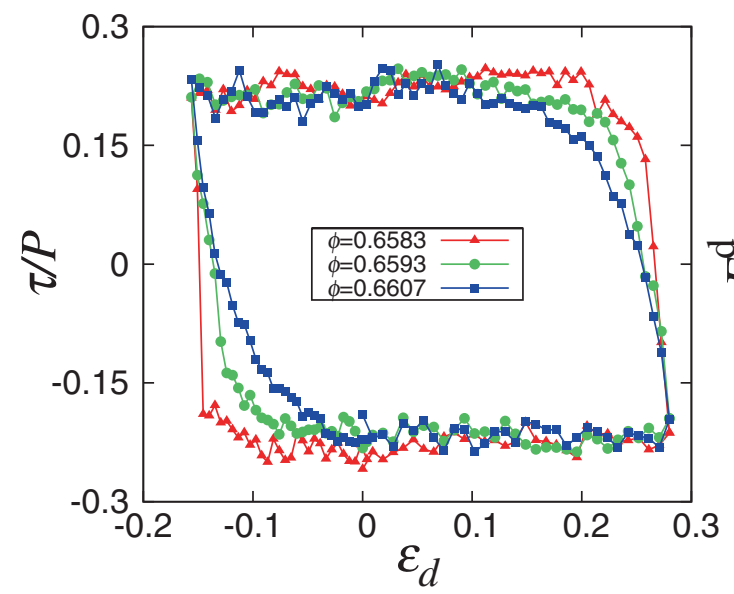

(e)

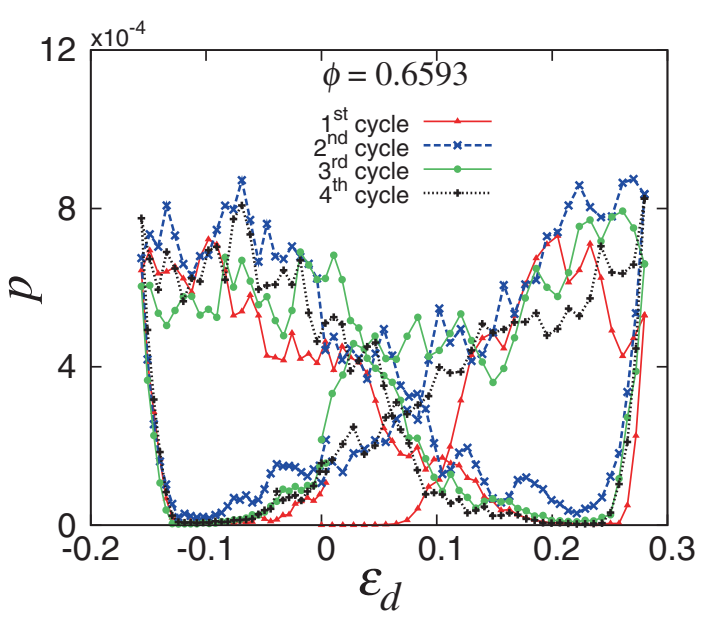

(b)

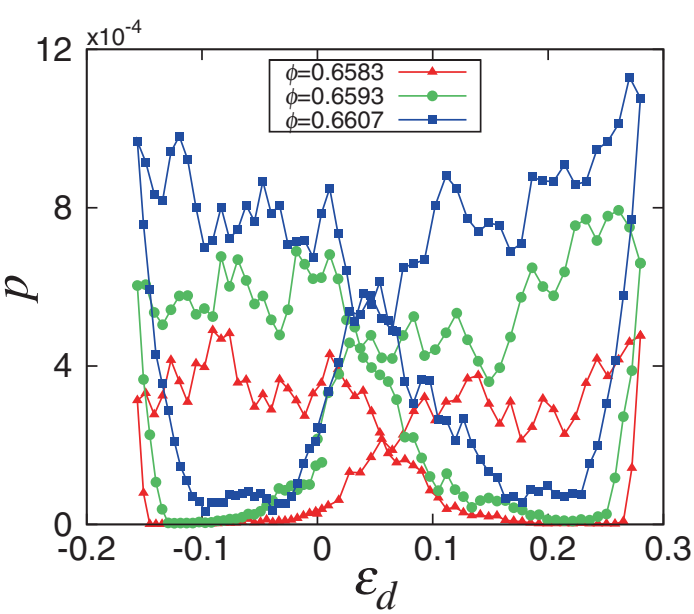

(d)

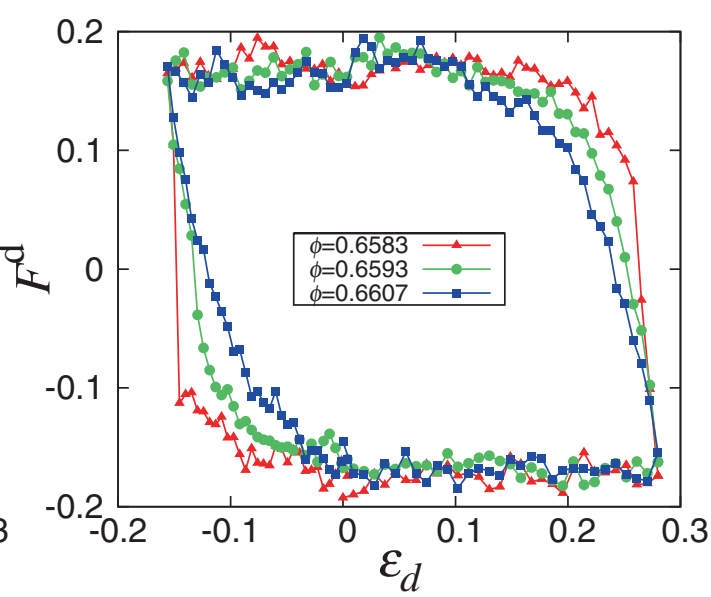

(f)

Figure 5.9: $(\mathrm{a}-\mathrm{b})$ Coordination number $C^{*}$ and pressure $p$ for the four shear cycles at constant volume fraction $\phi=0.6593$ and jamming point $\phi_{J}\left(\phi_{i}^{\max }=0.82, M=1\right)=:{ }^{1} \phi_{J, i}=0.6652$. (c-f) $C^{*}, p$, shear stress ratio $\tau / p$, and anisotropic fabric $F^{\mathrm{d}}$ for the second cycle only, for three different volume fractions $\phi$ as given in the insets. 
the second cycle. For higher volume fractions, we observe higher $C^{*}$ and $p$ limits, because of a higher probability for making contacts with the neighboring particles. Figs. 5.9e and 5.9f show the behavior of shear stress ratio, $\tau / p$ and deviatoric fabric $F^{\mathrm{d}}$ with strain $\varepsilon_{d}$ respectively. For higher shear strains, both $\tau / p \approx 0.22$ and $F^{\mathrm{d}} \approx 0.16$ saturate, with no visible effect of volume fraction, and similar characteristics for high opposite shear strains. However, their growth rates with applied strain are different. It is interesting to note that at reversal, the systems stay highly anisotropic in fabric and stress for some strain $\varepsilon_{d}$, whereas the coordination number $C^{*}$ and pressure $p$ drop down much more rapidly at reversal. After reversal, it also takes some finite strain for $C^{*}$ and $p$ to recover to $C^{*}>C_{0}^{*}=6$ and $p>0$.

\section{F Percolation analysis}

Here, we will discuss the percolation analysis, that allows to distinguish the three regimes namely, unjammed, fragile and shear jammed states during (and after) shear, as shown in Fig. 5.10a. We study how the $k$-cluster, defined as the largest force network, connecting strong forces, $f \geq k f_{\text {avg }}$, with $k=2.2$, percolates during shear. More quantitatively, for an exemplary volume fraction $\phi\left(\phi_{i}^{\max }=0.82, M=1\right)=0.6584$, very close to $\phi_{S J}$, Fig. $5.10 \mathrm{~b}$ shows that $f_{\mathrm{NR}}$ increases from initially zero to large values well below unity due to the always existing rattlers. The compressive direction percolating network $\xi_{y} / L_{y}$ grows faster than the tension direction network $\xi_{x} / L_{x}$, while the network in the non-mobile direction, $\xi_{z} / L_{z}$, lies in between them. For $f_{\mathrm{NR}}>0.82 \pm 0.01$, we observe that the growing force network is percolated in all three directions (Fig. 5.10a). The jamming by shear of the material corresponds (independently) to the crossing of $C^{*}$ from the isostatic limit of $C_{0}^{*}=6$, as presented in Fig. 5.10b.

Next, we present the evolution of the strong force networks in each direction during cyclic shear, as shown in Fig. 5.11, for the same initial system. After the first loading, at reversal $f_{\mathrm{NR}}$ drops below the 0.82 threshold, which indicates the breakage/disappearance of strong clusters, i.e. the system unjams. The new tension direction $\xi_{y} / L_{y}$ drops first with the network in the non-mobile directions, $\xi_{z} / L_{z}$, lying again in between the two mobile direction. With further applied strains, $f_{\mathrm{NR}}$ increases and again, the cluster associated with the compression direction grows faster than in the tension direction. For $f_{\mathrm{NR}}$ above the threshold, the cluster percolates the full system, leading to shear jammed states again. At each reversal, the strong force network breaks/fails in all directions, and the system gets "soft" or even un-jams temporarily. However, the network is rapidly is re-established in the perpendicular direction, i.e., the system jams and the strong, anisotropic force network again sustains the load. It is important to note that for some systems with volume fractions away from $\phi_{S J}$ can resist shear strain reversal (data not shown). 


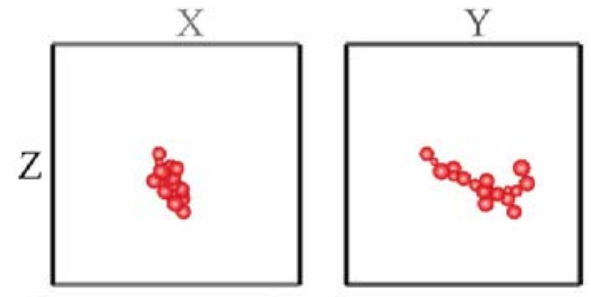

$\mathrm{f}_{\mathrm{NR}}=0.271 ;$ Unjammed states

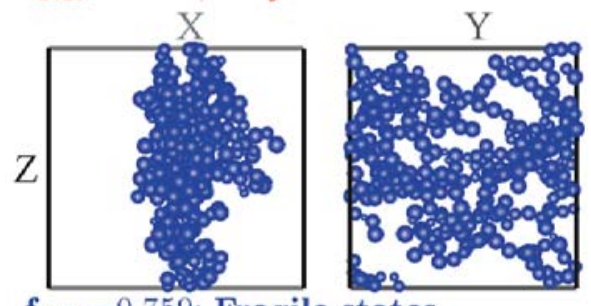

$\mathbf{f}_{\mathrm{NR}}=0.759 ;$ Fragile states

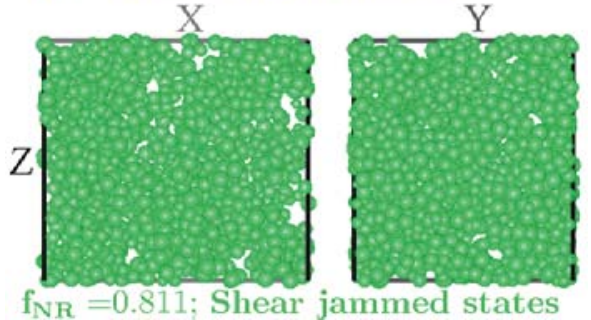

(a)

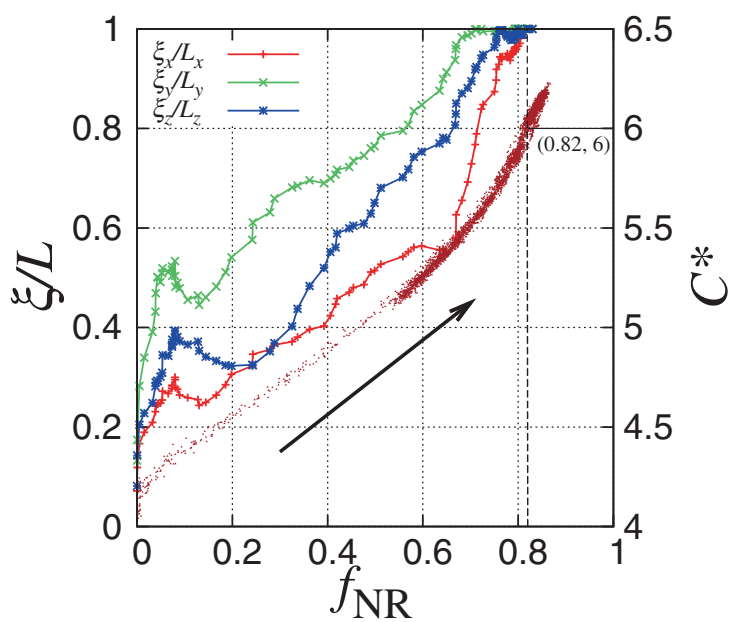

(b)

Figure 5.10: Evolution of the strong force networks during shear. (a) Plot of $C^{*}$ and cluster sizes $\xi / L$ in the three directions for tension in $x$ - and compression in $y$-directions against the non-rattler fraction $f_{\mathrm{NR}}$, along the loading path for an isotropic unjammed initial state with volume fraction $\phi=0.6584$ and $\phi_{J}\left(\phi_{i}^{\max }=0.82, M=1\right)=:{ }^{1} \phi_{J, i}=0.6652$. The upward arrow indicates the direction of loading shear strain. (b) Snapshots of unjammed, fragile and shear jammed states, when the force networks are percolated in none, one or two, and all the three directions, respectively. Only the largest force network, connecting strong forces, $f \geq k\langle f\rangle$, with $k \geq 2.2$ are shown for the three states for clarity. 


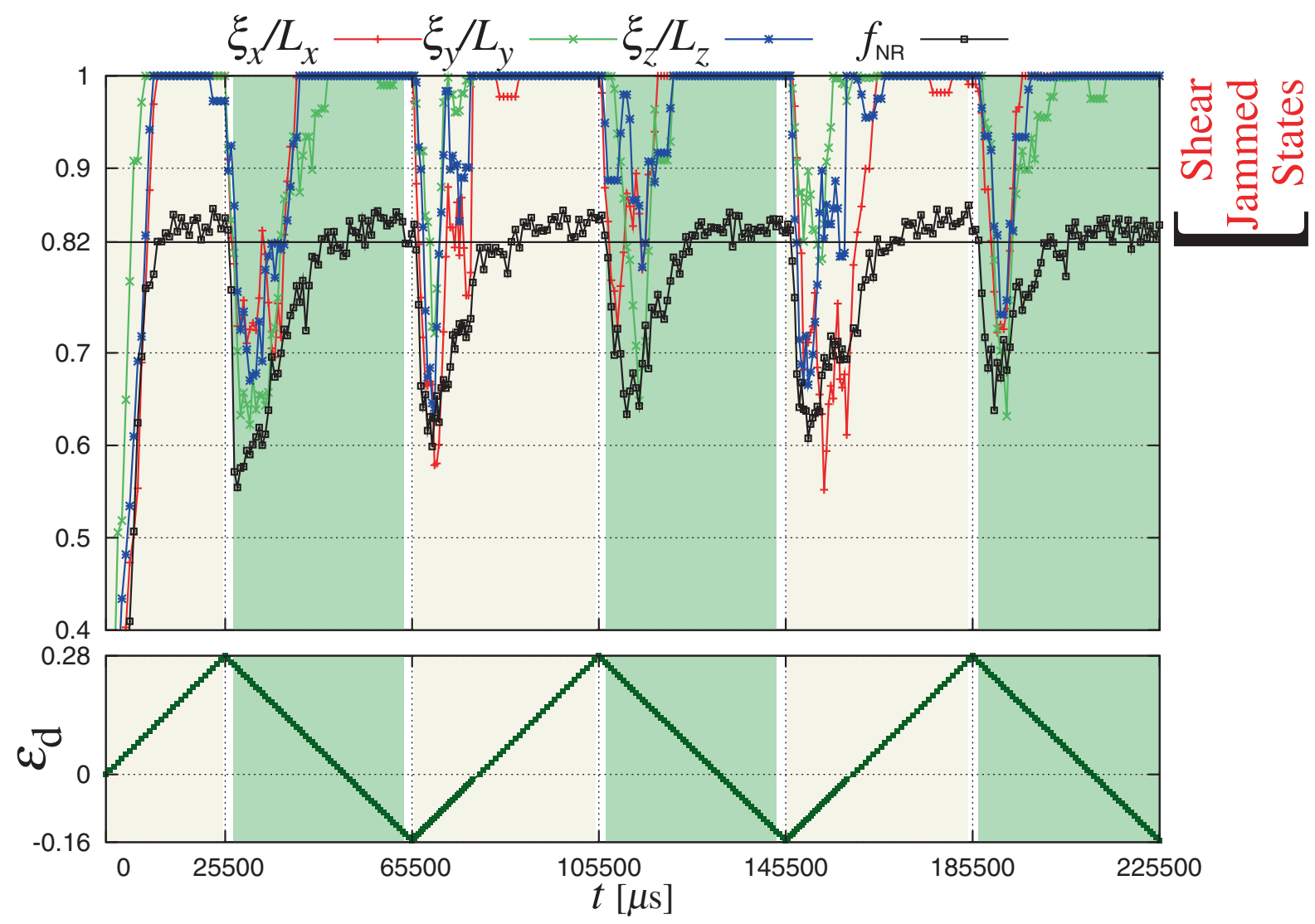

Figure 5.11: Cluster sizes, $f_{\mathrm{NR}}$ (top panel), over three strain cycles bottom for $\phi=$ 0.6584 and jamming point $\phi_{J}\left(\phi_{i}^{\max }=0.82, M=1\right)=:{ }^{1} \phi_{J, i}=0.6652$. The cluster sizes are smoothed averages over two past and future snapshots.

\section{G Relaxation effects}

In this section, we will discuss the system stability by looking at the macroscopic quantities in the saturation state (after large shear strain), by relaxing them sufficiently long to have nonfluctuating values in the microscopic and macroscopic quantities. Every shear cycle after defining e.g. the $y$-direction as the initial active loading direction, has two saturation states, one during loading and, after reversal, the other during unloading. In Fig. 5.12, we show values attained by the isotropic quantities pressure $p$, isotropic fabric $F_{\mathrm{v}}$ and the deviatoric quantities shear stress $\tau$, shear stress ratio $\tau / p$, and deviatoric fabric $F^{\mathrm{d}}$ for various $\phi$ given the same initial jamming point $\phi_{J}\left(\phi_{i}^{\max }=0.82, M=1\right)=:{ }^{1} \phi_{J, i}=0.6652$. Data are shown during cyclic shear as well as at the two relaxed saturation states (averaged over four cycles), leading to following observations:

(i) With increasing volume fraction, $p, F_{\mathrm{V}}$ and $\tau$ increase, while a weak decreasing trend in stress ratio $\tau / p$ and deviatoric fabric $F^{\mathrm{d}}$ is observed.

(ii) There is almost no difference in the relaxed states in isotropic quantities, $p$ and $F_{\mathrm{v}}$ for the two directions, whereas it is symmetric about zero for deviatoric quantities, $\tau, \tau / p$, and $F^{\mathrm{d}}$. 


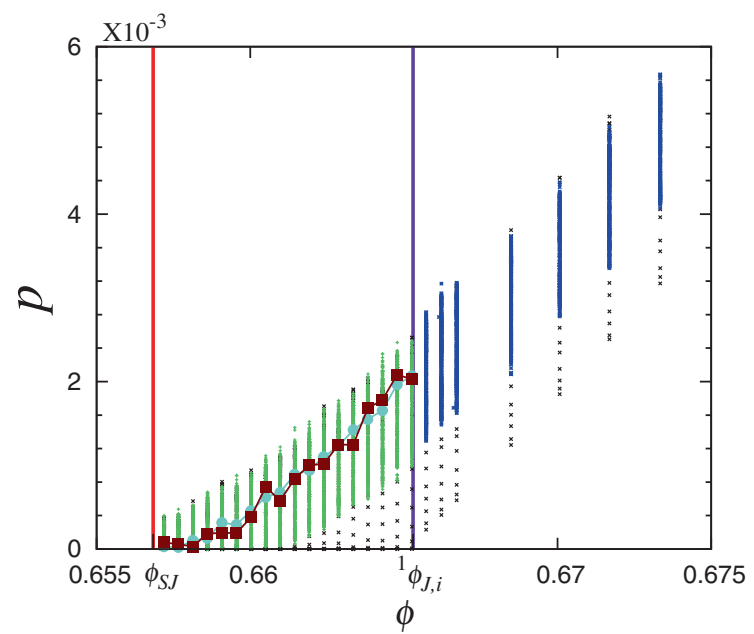

(a)

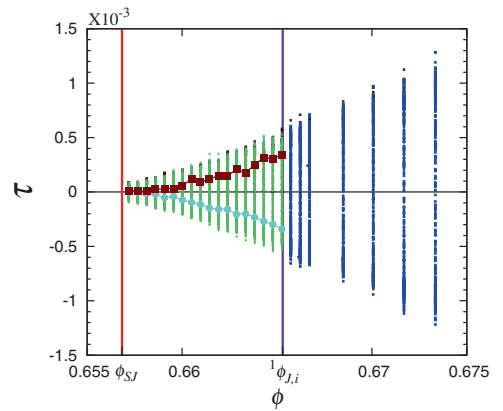

(c)

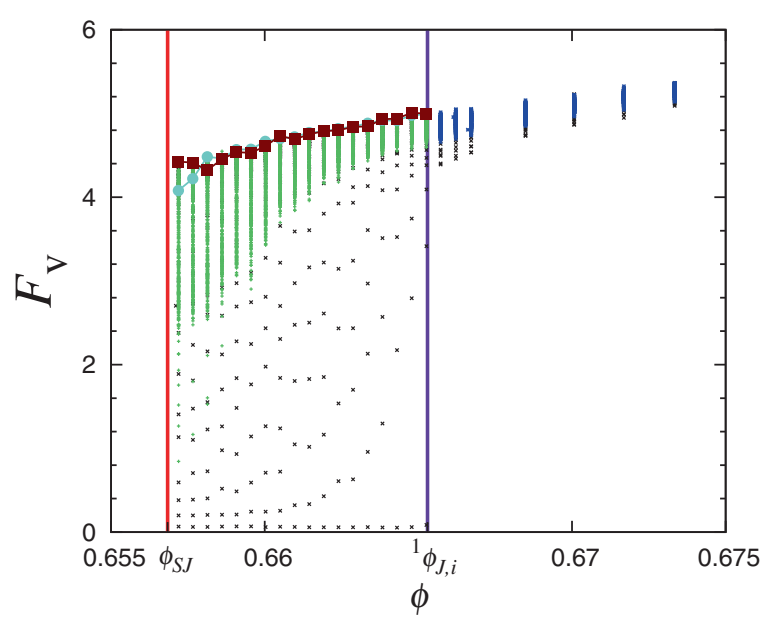

(b)

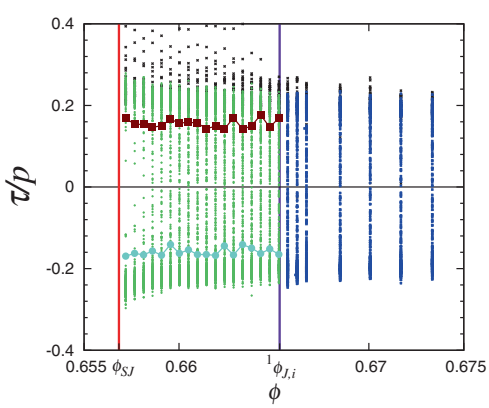

(d)

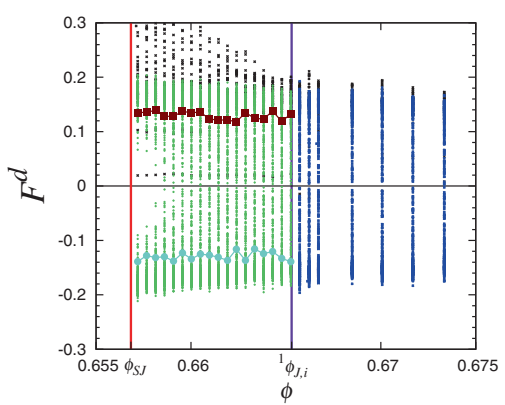

(e)

Figure 5.12: Scatter plots of isotropic quantities (a) pressure $p$, (b) isotropic fabric $F_{\mathrm{V}}$ and deviatoric quantities (c) shear stress $\tau$, (d) shear stress ratio $\tau / p$, and (e) deviatoric fabric $F^{\mathrm{d}}$ for various $\phi$ and jamming point $\phi_{J}\left(\phi_{i}^{\max }=0.82, M=1\right)=:{ }^{1} \phi_{J, i}=0.6652$. Black 'x' symbols represent the initial loading cycle, while the green ' + ' and blue ' $*$ ' represent states attained for $\phi<\phi_{J}$ and $\phi>\phi_{J}$, respectively for the subsequent shear. Cyan ' $\bullet$ ' and the brown 'ø' are states chosen after large strain during loading and unloading shear respectively, and are relaxed. The red and purple lines indicate the shear jamming point $\phi_{S J}=0.6567$ and the jamming point ${ }^{1} \phi_{J, i}$ respectively.

The decrease in pressure during relaxation is associated with dissipation of kinetic energy and partial opening of the contacts to "dissipate" the related part of the contact potential energy. However, $F_{\mathrm{v}}$ remains at its peak value during relaxation, showing that the contact structure is almost unchanged and the network remains stable during relaxation.

(iii) For small volume fractions, close to $\phi_{S J}$, the system becomes strongly anisotropic in stress ratio $\tau / p$, and fabric $F^{\mathrm{d}}$ rather quickly, during (slow) shear (envelope for low volume fractions in Figs. 5.12d and 5.12e), before it reaches the steady state.

(iv) It is easy to obtain the shear jamming point $\phi_{S J}$ from the relaxed critical (steady) state pressure $p$, and shear stress $\tau$, by extrapolation to zero, as the envelope of relaxed data in Figs. 5.12a and 5.12c. 
We use the same methodology using Eq. (5.4), to extract the shear jamming point $\phi_{S J}$. When the relaxed $p$ is normalized with the contact density $\phi C$, we obtain $\phi_{S J}=0.6567 \pm 0.0005$ by linear extrapolation. A similar value of $\phi_{S J}$ is obtained from the extrapolation of the relaxed $\tau$ data set, and is consistent with other methods using the coordination number $C^{*}$, or the energy [70].

\section{H Predictive power}

In this section, we present the simplest model equations, as used for the predictions presented in Fig. 5.4, involving a history dependent $\phi_{J}(H)$, as given in Eq. (5.1) for isotropic deformations and Eq. (5.3) for shear deformations. The only difference to Ref. [84], where these relations are taken from, based on purely isotropic unloading, is a variable $\phi_{J}=\phi_{J}(H)$.

During (cyclic) isotropic deformation, the evolution equation for the corrected coordination number $C^{*}$ is:

$$
C^{*}=C_{0}+C_{1}\left(\frac{\phi}{\phi_{J}(H)}-1\right)^{\theta},
$$

with $C_{0}=6$ for the frictionless case and parameters $C_{1}$ and $\theta$ are presented in Table 5.1. The fraction of non-rattlers $f_{\mathrm{NR}}$ is given as:

$$
f_{\mathrm{NR}}=1-\varphi_{c} \exp \left[-\varphi_{v}\left(\frac{\phi}{\phi_{J}(H)}-1\right)\right],
$$

with parameters $\varphi_{c}$ and $\varphi_{v}$ presented in Table 5.1. We modify Eq. (5.4) for the evolution of $p$ together with the history dependent $\phi_{J}=\phi_{J}(H)$ so that,

$$
p=\frac{\phi C}{\phi_{J}(H)} p_{0}\left(-\varepsilon_{\mathrm{V}}\right)\left[1-\gamma_{p}\left(-\varepsilon_{\mathrm{v}}\right)\right]
$$

with parameters $p_{0}$ and $\gamma_{p}$ presented in Table 5.1, and the true or logarithmic volume change of the system is $-\varepsilon_{\mathrm{v}}=\log \left(\phi / \phi_{J}(H)\right)$, relative to the momentary jamming density. The noncorrected coordination number is $C=C^{*} f_{\mathrm{NR}}$, as can be computed using Eqs. (5.7) and (5.8). The isotropic fabric $F_{\mathrm{v}}$ is given by the relation $F_{\mathrm{v}}=g_{3} \phi C$, as taken from Imole et al. [84], with $g_{3} \cong 1.22$ for the polydispersity used in the present work. Also the parameters $C_{1}, \theta$ for $C^{*}, \varphi_{c}, \varphi_{v}$ for $f_{\mathrm{NR}}$; and $p_{0}, \gamma_{p}$ for pressure $p$ are similar to Ref. [84], with the second order correction parameter $\gamma_{p}$ most sensitive to the details of previous deformations; however, not being very relevant since it is always a small correction due to the product $\gamma_{p}\left(-\varepsilon_{\mathrm{v}}\right)$.

The above relations are used to predict the behavior of the isotropic quantities: dimensionless pressure $p$ and coordination number $C^{*}$, as shown in Fig. 5.4(a-b) during isotropic compression, as well as for the fraction of non-rattlers in Fig. 5.4c for cyclic shear, with corresponding parameters presented in Table 5.1. Note that during isotropic deformation, $\phi_{J}(H)$ was 


\begin{tabular}{c|cc}
\hline \hline \multirow{2}{*}{ Quantity } & Isotropic & Shear \\
\hline \hline & $C_{1}=8.5^{*} ; \theta=0.58$ & $C_{1}=8.1 ; \theta=0.58$ \\
$C^{*}$ & $\varphi_{c}=0.13 ; \varphi_{v}=15$ & $\varphi_{c}=0.16 ; \varphi_{v}=15$ \\
$f_{\mathrm{NR}}$ & $p_{0}=0.042 ; \gamma_{p}=0 \pm 0.1^{*}$ & $p_{0}=0.042 ; \gamma_{p}=0 \pm 0.1 *$
\end{tabular}

Table 5.1: Parameters used in Eqs. (5.7), (5.8) and (5.9), where '*' represents slightly different values than from Ref. [84].

changed only during the compression branch, using Eq. (5.1) for fixed $M=1$ using $\phi_{i}^{\max }$ as variable, but is kept constant during unloading/expansion.

During cyclic (pure) shear deformation, a simplified equation for the shear stress ratio $\tau / p$ is taken from Imole et al. [84] as:

$$
\tau / p=(\tau / p)^{\max }-\left[(\tau / p)^{\max }-(\tau / p)^{0}\right] \exp \left[-\beta_{s} \varepsilon_{d}\right],
$$

with $(\tau / p)^{0}$ and $(\tau / p)^{\max }$ the initial and maximum (saturation) shear stress ratio, respectively, and $\beta_{s}$ its growth rate. Similarly, a simplified equation for the deviatoric fabric $F^{\mathrm{d}}$ can be taken from Imole et al. [84] as:

$$
F^{\mathrm{d}}=F^{\mathrm{d}^{\max }}-\left[F^{\mathrm{d}^{\max }}-F^{\mathrm{d}^{0}}\right] \exp \left[-\beta_{F} \varepsilon_{d}\right]
$$

with $F^{\mathrm{d}^{0}}$ and $F^{\mathrm{d}^{\max }}$ the initial and maximum (saturation) values of the deviatoric fabric, respectively, and $\beta_{F}$ its growth rate. The four parameters $(\tau / p)^{\max }, \beta_{s}$ for $\tau / p$ and $F^{\mathrm{d}^{\mathrm{max}}}$, $\beta_{F}$ for $F^{\mathrm{d}}$ are dependent on the volume fraction $\phi$ and are well described by the general relation from Imole et al. [84] as:

$$
Q=Q_{a}+Q_{c} \exp \left[-\Psi\left(\frac{\phi}{\phi_{J}(H)}-1\right)\right],
$$

where $Q_{a}, Q_{c}$ and $\Psi$ are the fitting constants with values presented in Table 5.2.

For predictions during cyclic shear deformation, $\phi_{J}(H)$ was changed with applied shear strain $\varepsilon_{d}$ using Eq. (5.3). Furthermore, the jamming point is set to a larger value just after strain-reversal which is discussed next.

\section{Behavior of jamming point at strain reversal}

As mentioned in section 5.F, there are some states below $\phi_{J}$, where application of shear strain jams the systems. The denses of those can resist shear reversal, but below a certain 


\begin{tabular}{clll}
\hline \hline Evolution parameters & $Q_{a}$ & $Q_{c}$ & $\Psi$ \\
\hline \hline$(\tau / p)^{\max }$ & 0.12 & 0.091 & 7.9 \\
$\beta_{s}$ & 30 & 40 & 16 \\
$F^{\mathrm{d}^{\max }}$ & 0 & 0.17 & 5.3 \\
$\beta_{F}$ & 0 & 40 & 5.3 \\
\hline \hline
\end{tabular}

Table 5.2: Parameters for Eqs. (5.10) and (5.11) using Eq. (5.12), with slightly different values than from Ref. [84], that are extracted using the similar procedure as in [84], for states with volume fraction close to the jamming volume fraction.

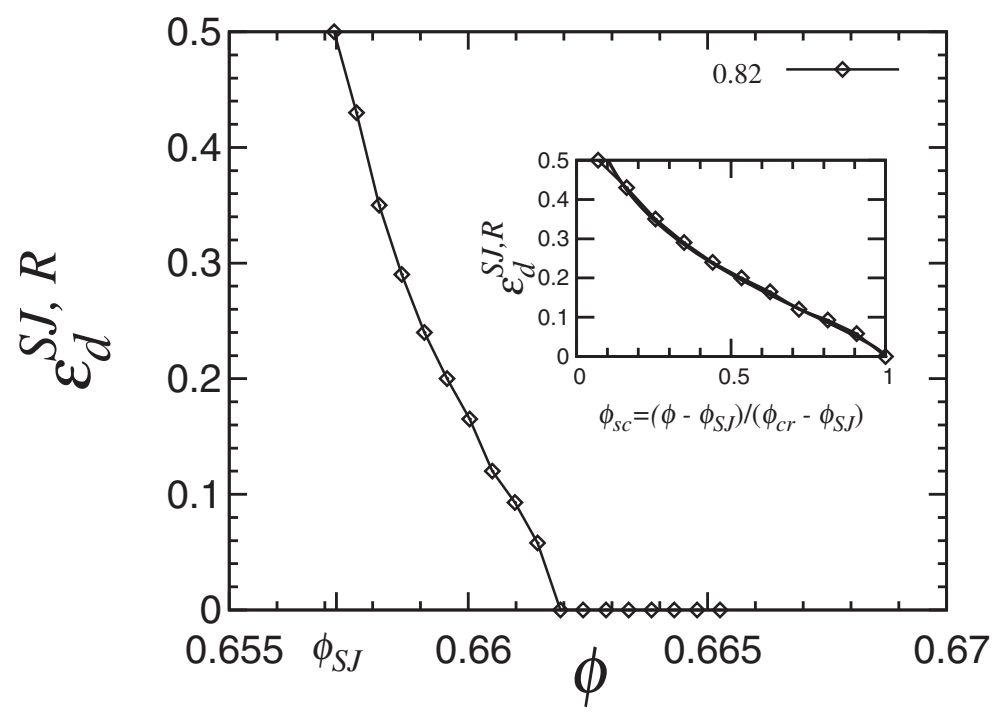

Figure 5.13: Phase diagram showing the minimum reversal shear strain $\varepsilon_{d}^{S J, R}$ needed to jam the states below $\phi_{\mathrm{cr}}$, for states prepared from the first over-compression cycle with different $\phi_{i}^{\max }$, as given in the legend. Inset shows collapse of the states using a similar scaled definition as Eq. (5.2), that includes distance from both $\phi_{\text {cr }}$ and shear-jamming point $\phi_{S J}$, using Eq. (5.13).

$\phi_{\mathrm{cr}}<\phi_{J}$, shear reversal unjams the system again. With this information, we postulate the following:

(i) After the first large strain shear, the system should forget, where it was isotropically compressed to before i.e., ${ }^{M} \phi_{J, i}$ if forgotten and $\phi_{J}=\phi_{S J}$ is realized.

(ii) There exists a critical volume fraction $\phi_{\mathrm{cr}}$, above which systems can just resist shear reversal and remain always jammed in both forward and reverse shear.

(iii) Below this $\phi_{\mathrm{cr}}$, reversal unjams the system. Therefore, more strain is needed to jam the system (when compared to the initial loading), first to forget its state before reversal, and 
then to re-jam it in opposite (perpendicular) direction. Hence, the strain necessary to jam in reversal direction should be higher than for the first shear cycle.

(iv) As we approach $\phi_{S J}$, the reverse strain needed to jam the system increases.

We use these ideas and measure the reversal shear strain $\varepsilon_{d}^{S J, R}$, needed to re-jam the states below $\phi_{\mathrm{cr}}$, as shown in Fig. 5.13. When they are scaled with $\phi_{\mathrm{cr}}$ as $\phi_{s c}=\left(\phi-\phi_{S J}\right) /\left(\phi_{\mathrm{cr}}-\phi_{S J}\right)$ , they collapse on a unique master curve, very similar to Eq. (5.2) :

$$
\left(\varepsilon_{d}^{S J, R} / \varepsilon_{d}^{0, R}\right)^{\alpha}=-\log \phi_{S c}=-\log \left(\frac{\phi-\phi_{S J}}{\phi_{\mathrm{cr}}-\phi_{S J}}\right)
$$

shown in the inset of Fig. 5.2b, with the same power $\alpha=1.37 \pm 0.01$ as Eq. (5.2). Fit parameter strain scale $\varepsilon_{d}^{0, R}=0.17 \pm 0.002>\varepsilon_{d}^{0}=0.102$, is consistent with the above postulates (iii) and (iv).

The above relations are used to predict the isotropic and the deviatoric quantities, during cyclic shear deformation, as shown in Fig. 5.4c, with the additional rule that all the quantities attain value zero for $\phi \leq \phi_{J}(H)$. Moreover, for any state with $\phi \leq \phi_{\mathrm{cr}}$, shear strain reversal moves the jamming point to $\phi_{\mathrm{cr}}$, and the movement of jamming point follows Eq. (5.13).

Any other deformation mode, can be written of as a unique superposition of pure isotropic and pure shear deformation modes, and hence the combination of the above can be easily used to describe any general deformation, e.g. uniaxial cyclic compression (data not presented).

\section{I How to measure $\phi_{J}$ from experiments}

Last, but not the least, we conclude by plotting the history dependent jamming point $\phi_{J}(H)$ from measurable quantities, indirectly obtained via Eqs. (5.3), (5.8), (5.9), and directly from Eq. (5.3). There are two reasons to do so: $(i)$ the jamming point $\phi_{J}(H)$ is only accessible in the unloading limit $p \rightarrow 0$, which requires an experiment or a simulation to "measure" it (however, during this measurement, it might change again); (ii) deducing the jamming point from other quantities that are defined for an instantaneous snapshot/configuration for $p>0$ allows to indirectly obtain it - if, as shown next, these indirect "measurements" are compatible/consistent: Showing the equivalence of all the different $\phi_{J}(H)$, proofs the consistency and completeness of the model and, even more important, provides a way to obtain $\phi_{J}(H)$ indirectly from experimentally accessible quantities.

\section{For isotropic compression}

Fig. 5.14 shows the evolution of $\phi_{J}(H)$, measured from the two experimentally accessible quantities: coordination number $C^{*}$ and pressure $p$, using Eqs. (5.7) and (5.9) respectively for isotropic over-compression to $\phi_{i}^{\max }=0.82$ over two cycles. Following observations 


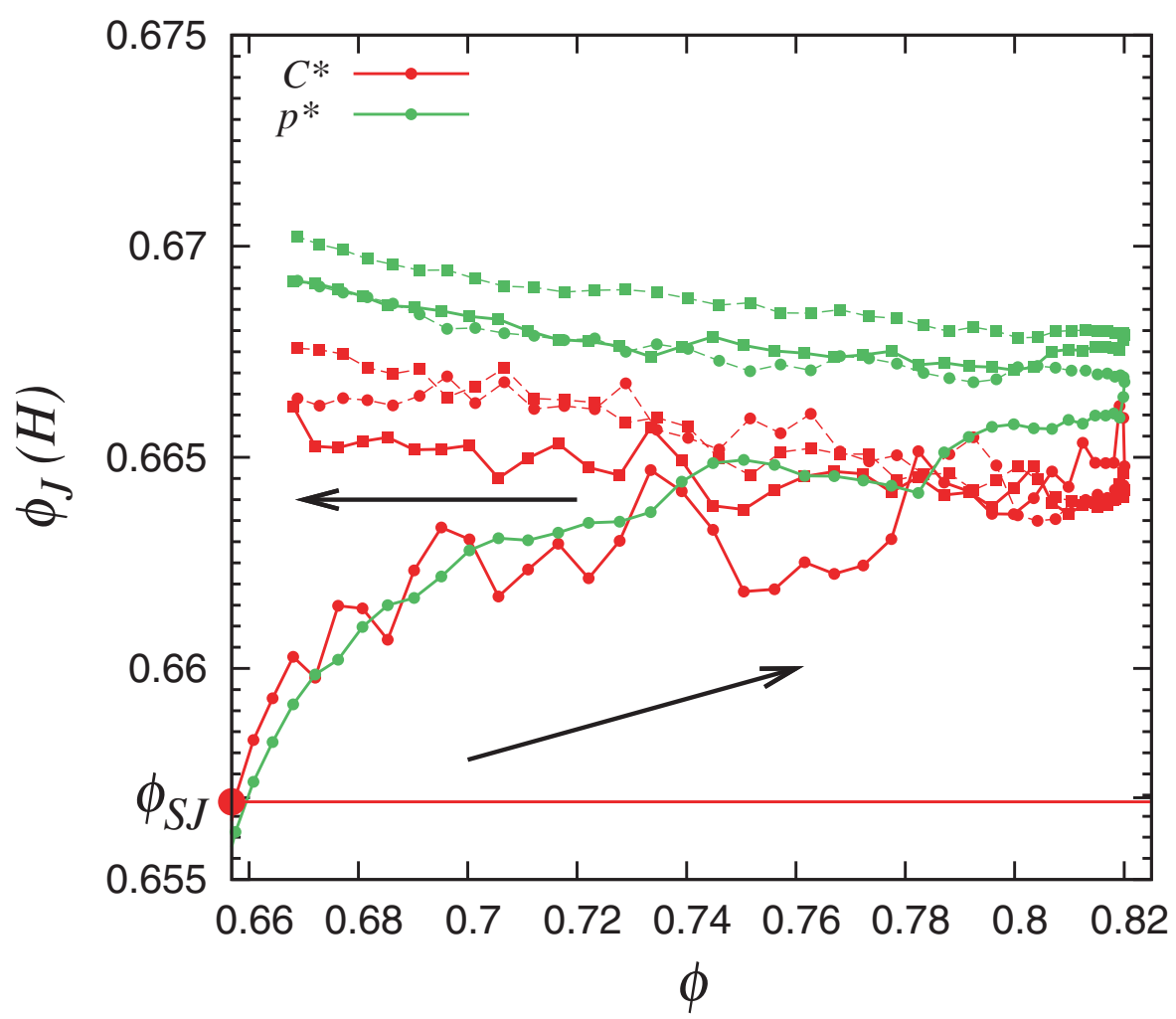

(a)

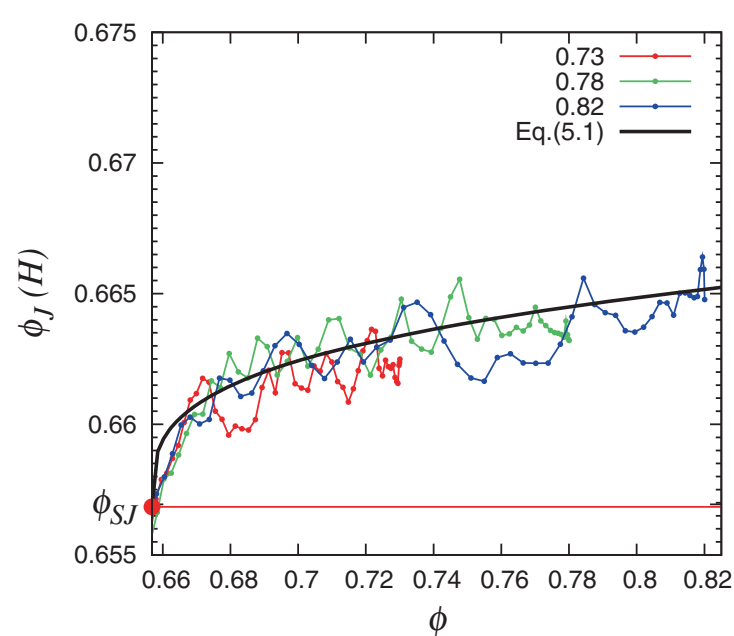

(b)

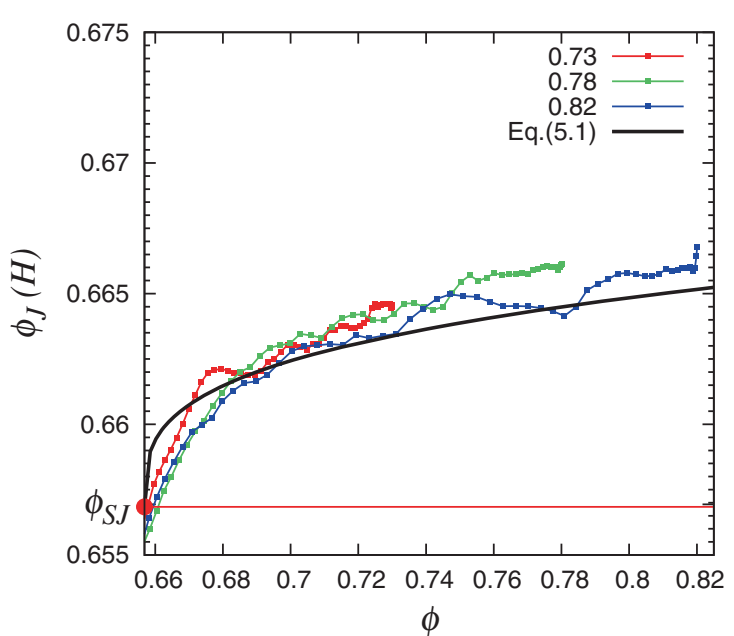

(c)

Figure 5.14: (a) Evolution of the history dependent jamming point $\phi_{J}(H)$ during isotropic over-compression to $\phi_{i}^{\max }=0.82$ for two cycles, calculated back from the measured quantities: coordination number $C^{*}$ (green) and pressure $p$ (red), using Eqs. (5.7) and (5.9) respectively. The ' $\bullet$ ' and ' $\boldsymbol{\square}$ ' represent the first and second cycle respectively. Solid lines are the loading path while the dashed lines represent the unloading path for the corresponding cycle. Evolution of history dependent jamming point $\phi_{J}(H)$ using (b) coordination number $C^{*}$ and (c) pressure $p$ for three levels of over-compression, as shown in the inset. Solid black line represents Eq. (5.1) with $M=1$, and ${ }^{\infty} \phi_{J, i}$ calculated using Eq. (5.5). 


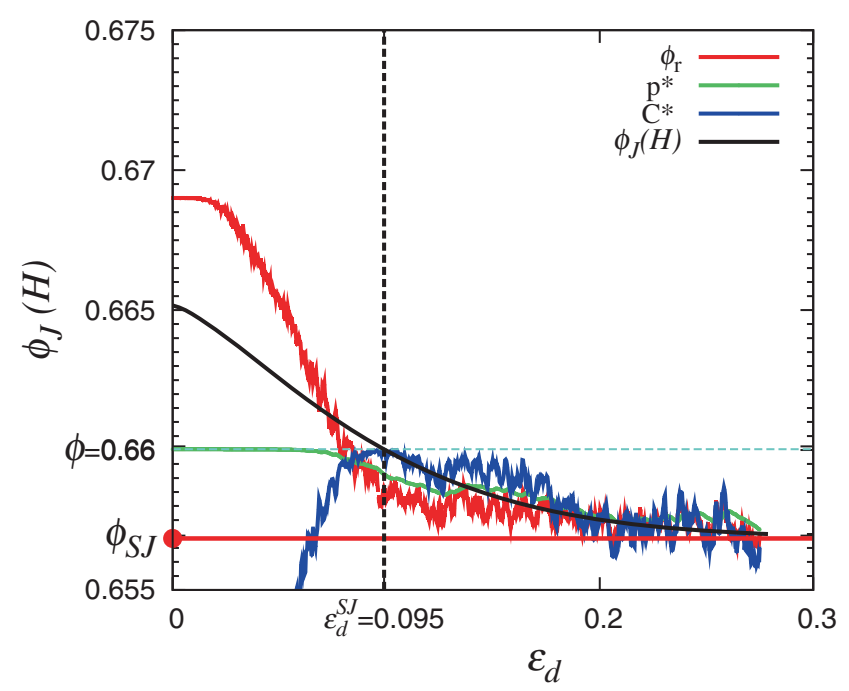

Figure 5.15: Evolution of the history dependent jamming point $\phi_{J}(H)$ during pure shear, calculated back from the measured quantities: coordination number $C^{*}$, fraction of nonrattlers $f_{\mathrm{NR}}$ and pressure $p$, using Eqs. (5.7), (5.8), (5.9) respectively, as indicated in the legend. The volume fraction is constant, $\phi=0.66$, and the initial jamming point $\phi_{J}\left(\phi_{i}^{\max }=0.82, M=1\right)=:{ }^{1} \phi_{J, i}=0.6652$ is greater than $\phi$ (represented by horizontal cyan line). The solid black line represents Eq. (5.3), and the dashed vertical line indicates the shear strain needed to jam the system, $\varepsilon_{d}^{S J}$, from which on - for larger shear strain - the system is jammed.

can be made: $(i) \phi_{J}$ for isotropic loading and unloading can be extracted from $C^{*}$ and $p$; (ii) it rapidly increases and then saturates during loading.; (iii) it mimics the fractal energy landscape model in Fig. 4 from Ref. [127] very well; (iv) while is was assumed not to change for unloading, it even increases, which we attribute to the perturbations and fluctuations (granular temperature) induced during the quasi-static deformations; $(v)$ the indirect $\phi_{J}$ are reproducible and follow the same master-curve for first over-compression as seen in Figs. 5.14 , independent of the maximum - all following deformation is dependent on the previous maximum density..

\section{For shear deformation}

Fig. 5.15 shows the evolution of $\phi_{J}(H)$, measured from the two experimentally accessible quantities: coordination number $C^{*}$ and pressure $p$, using Eqs. (5.7) and (5.9) respectively during volume conserving shear with $\phi=0.66$, and the initial jamming point $\phi_{J}\left(\phi_{i}^{\max }=0.82, M=1\right)=:{ }^{1} \phi_{J, i}=0.6652>\phi$ and shows good agreement with the theoretical predictions using Eq. (5.3) after shear jamming. Thus the indirect measurements of $\phi_{J}(H)$ can be applied if $\phi_{J}(H)<\phi$; the result deduced from pressure fits the best, i.e., it interpolates the two others and is smoother. 


\section{CHAPTER 6 \\ Conclusions and Recommendations}

\section{Conclusions}

Understanding the mechanical properties of granular materials is important for both practical and fundamental reasons. In the early stage of this dissertation, we have studied the constitutive behavior of idealized granular materials modeled as random polydisperse sphere packings using the discrete element method. We focused on strain-controlled loading and unloading of isotropic, uniaxial and two deviatoric (pure shear) type deformation modes. Experimentally most difficult to realize is the isotropic deformation, while both uniaxial and deviatoric modes can be realized in different element test set-ups where, however, often mixed strain- and stress-control is applied. Both uniaxial and deviatoric modes can be realized experimentally, in different element test set-ups where mixed strain- and stress-control is often applied, however it is an arduous task to realize the isotropic deformations experimentally. Several noteworthy conclusions can be drawn from the analysis:

For idealized soft, frictionless, polydisperse granular systems discussed in Chapters 2 and 3:

1. The corrected coordination number increases while rattler fraction decreases with volume fraction following a power law above the jamming volume fraction for all deformation modes. The coordination number decreases with polydispersity (with uniform particle size distribution), while the fraction of rattlers displays an opposite trend. At small strains, the uniaxial, deviatoric (volume conserving) and isotropic modes can be described by the same analytical pressure evolution for the linear contact model for different polydispersity. 
2. The jamming volume fraction is not a single value for a particular system configuration, but it is strongly dependent on the deformation mode and history of the packing. The jamming volume fraction increases with polydispersity, with the isotropic and deviatoric tests giving the highest and lowest values, respectively, while the uniaxial dataset lies in between them.

3. During deviatoric and uniaxial deformations, both deviatoric stress ratio and deviatoric fabric evolve with the deviatoric strain. The saturation value in deviatoric quantities during deviatoric deformations increase with polydispersity.

4. The micro-macro observations from the pure deformations - isotropic and deviatoric tests - can be used to calibrate a simple constitutive model involving anisotropy, and is valid for range of polydispersity. The (overly) simple material (model) allows to focus on the collective/bulk response of the material to different types of strain. The calibrated model is able to predict both stress and fabric evolution under uniaxial deformation - with very good qualitative success and within 70-80\% quantitative agreement.

For the calibration of anisotropy continuum model, we presented the methodology to first extract the elastic moduli of granular materials by applying purely isotropic and deviatoric perturbations and the relationship of moduli with the present stress and fabric state. The main conclusions of the study, discussed in Chapter 4 are:

5. The bulk modulus only depends on the isotropic contact network (isotropic fabric). The ratios of the two (different) anisotropy moduli with the bulk modulus is related only to the deviatoric fabric, as the whole contact network determines how the system will react to a further perturbation. The shear modulus is linked with both the contact network and the stress state of the granular assembly. This approach can also be extended to various particle size distributions e.g., lognormal and Weibull distributions.

6. The calibrated anisotropy continuum model is able to quantitatively predict the evolution of pressure, shear stress and fabric using the relations between elastic moduli and stress and microstructure.

Finally, a simple, unified, universal quantitative model is proposed for predicting the micromacro behavior of three-dimensional soft, frictionless, polydisperse granular assemblies. The key ingredient is the knowledge of how the jamming density changes with sample's history. The main conclusions from Chapter 5 are:

7. The packing efficiency can increase logarithmically slow (creep) under gentle "tapping" or repeated over-compression, leading to an increase of the jamming density. Shear deformations cause re-structuring, anisotropy and also dilatancy, and rapidly decrease the jamming density.

8. There exists an absolute minimum jamming volume fraction, that represents the critical (steady) state in the limit of vanishing confining stress, i.e., the true jamming-point. 
This is nothing else than the lowest stable random density a sheared system locally can reach due to continuously ongoing shear, for vanishing confining stress. This knowledge of change of jamming density deformation mode (history) can be cast into a simple model involving history.

\section{Recommendations}

The work in this thesis is undertaken as a step forward towards understanding the relationship between microscale particle properties and macroscopic behavior, e.g., the jamming transition in granular materials. There are several limitations and many aspects that require further research.

The general framework and guidelines for jamming transition study, as described here, has been established using idealized, polydisperse granular materials, interacting with linear spring contact models. This simple approach is able to capture important features of granular materials, however simulations with more realistic contact models such as Hertz-Mindlin should be performed to generalize the results of this thesis for the case of realistic elastic or elasto-plastic spheres.

More realistic particle simulations aimed for a systematic understanding of the effects of friction and cohesion, should be performed, using the findings from this thesis as a basis. The samples considered were almost homogeneous in stress, fabric and strain-rate. However, it is not the case when real materials are handled, therefore, another possible direction for further research is to model granular assemblies comprising heterogeneous compositions and behaviors.

The elastic moduli measured in the present study were limited to four, whereas, the general stiffness matrix has at least six parameters. A variety of small strain perturbations in the present study were performed using the pure shear deformation mode, to exclude the effect of volume fraction, and gather a statistically stable dataset for different volume fractions. Further work should be done to characterize the full elastic behavior of the material, with focus to extract them by performing least number of experiments, as performing numerous small strain perturbations in experiments is a cumbersome process. Effect on these measurements from a view of sample's size should be done in the future.

In this study, a constitutive model involving anisotropy was used, when calibrated carefully, predicts the cyclic shear deformation test quantitatively. Comparison with other continuum models like elasto-plasticity, hypoplastic or GSH should be made to obtain predictions of other general arbitrary deformation paths. Elasticity itself is a very interesting topic that we explored in this dissertation and can be effectively addressed following the methodology presented. Furthermore, the simulation results should be checked against available experimental results and if required more appropriate experiments should be carried out. Some parameters 
such as the coordination number are not easily accessible in the experiments. Therefore, the use of advanced imaging techniques like X-ray tomography may be an advantage for this purpose.

Finally, more experiments should be done to validate our model that requires the dynamics of jamming point changes, as the main ingredient, based on the sample history. The accessible range of difference in current jamming and the lowest shear jamming point is expected to further increase for more realistic systems, e.g., with friction, or non-spherical particle shapes. Some experimental and numerical measurements of the landscape valley width, depths and shapes should be performed to verify our our conjecture, as this remains qualitative so far. 


\section{References}

[1] The images are taken from:

http://fin6.com/2013/12/coffee-beans/,

http://en.wikipedia.org/wiki/bean/,

http://en.wikipedia.org/wiki/segregated_cycle_facilities/,

http://naturetime.wordpress.com/2013/01/09/, Accessed on 2014-02-20.

[2] The images are taken from:

http://jenike.com/structuralmechanical/inspectionsfailure-analysis/,

http://www.flickr.com/photos/cdeimages/6256463397/,

http://fr.wikipedia.org/wiki/rail/,

https://nsidc.org/cryosphere/frozenground/people.html, Accessed on 2014-02-20.

[3] I. Agnolin and J.-N. Roux. Internal states of model isotropic granular packings. I. Assembling process, geometry, and contact networks. Phys. Rev. E, 76(6, Part 1), 2007.

[4] J. Ai, J.-F. Chen, and J. Y. Ooi. Finite element simulation of the pressure dip in sandpiles. International Journal of Solids and Structures, 50(6):981-995, 2013.

[5] F. Alonso-Marroquin, S. Luding, H. J. Herrmann, and I. Vardoulakis. Role of anisotropy in the elastoplastic response of a polygonal packing. Phys. Rev. E, 71(5), 2005.

[6] Anderson. Influence of fines on performance on asphalt concrete mixtures. In Proceedings ICAR 4th Annual Symposium, 1996.

[7] A. D. Araújo, E. J. R. Parteli, T. Pöschel, J. S. Andrade, and H. J. Herrmann. Numerical modeling of the wind flow over a transverse dune. Scientific reports, 3, 2013.

[8] S. S. Ashwin, M. Zaeifi Yamchi, and R. K. Bowles. Inherent Structure Landscape Connection between Liquids, Granular Materials, and the Jamming Phase Diagram. Phys. Rev. Lett., 110(14):145701, 2013.

[9] E. Azéma and F. Radjaï. Force chains and contact network topology in sheared packings of elongated particles. Phys. Rev. E, 85(3), 2012.

[10] E. Azéma, F. Radjai, and G. Saussine. Quasistatic rheology, force transmission and fabric properties of a packing of irregular polyhedral particles. Mechanics of Materials, 41(6):729-741, 2009.

[11] K. Bagi. Stress and strain in granular assemblies. Mechanics of Materials, 22(3):165-177, 1996.

[12] M. M. Bandi, M. K. Rivera, F. Krzakala, and R. E. Ecke. Fragility and hysteretic creep in frictional granular jamming. Phys. Rev. E, 87(4), 2013. 
[13] E. J. Banigan, M. K. Illich, D. J. Stace-Naughton, and D. A. Egolf. The chaotic dynamics of jamming. Nat Phys, 9(5):288-292, 2013.

[14] J. P. Bardet and I. Vardoulakis. The asymmetry of stress in granular media. International Journal of Solids and Structures, 38(2):353-367, 2001.

[15] D. Barreto and C. O'Sullivan. The influence of inter-particle friction and the intermediate stress ratio on soil response under generalised stress conditions. Granular Matter, 14(4):505-521, 2012.

[16] E. Bauer, W. Huang, and W. Wu. Investigations of shear banding in an anisotropic hypoplastic material. International Journal of Solids and Structures, 41(21):5903-5919, 2004.

[17] M. Belkhatir, A. Arab, N. Della, and T. Schanz. Experimental Study of Undrained Shear Strength of Silty Sand: Effect of Fines and Gradation. Geotechnical and Geological Engineering, 30(5):1103-1118, 2012.

[18] M. Belkhatir, A. Arab, T. Schanz, H. Missoum, and N. Della. Laboratory study on the liquefaction resistance of sand-silt mixtures: effect of grading characteristics. Granular Matter, 13(5):599-609, 2011.

[19] R. Bellotti, M. Jamiolkowski, D. A. O’Neill, and D. C. F. L. Presti. Anisotropy of small strain stiffness in Ticino sand. Géotechnique, 46(1):115-131, 1996.

[20] Belyaeva, Zaitsev, and L. A. Ostrovskii. Nonlinear acoustoelastic properties of granular media. Acoust. Phys., 39(1):11-15, 1993.

[21] D. Bi, J. Zhang, B. Chakraborty, and R. P. Behringer. Jamming by shear. Nature, 480(7377):355-358, 2011.

[22] G. Biroli. Jamming - A new kind of phase transition? Nature Physics, 3(4), 2007.

[23] E. Brown and H. Jaeger. Dynamic Jamming Point for Shear Thickening Suspensions. Phys. Rev. Lett., 103(8):086001, 2009.

[24] R. L. Brown and P. G. W. Hawksley. Packing of Regular (Spherical) and Irregular Particles. Nature, 156:421$422,1945$.

[25] J. Brujić, C. Song, P. Wang, C. Briscoe, G. Marty, and H. A. Makse. Measuring the Coordination Number and Entropy of a 3D Jammed Emulsion Packing by Confocal Microscopy. Phys. Rev. Lett., 98(24), 2007.

[26] F. Calvetti, G. Viggiani, and C. Tamagnini. A numerical investigation of the incremental behavior of granular soils. Rivista Italiana di Geotecnica, 37(3):11-29, 2003.

[27] J. W. Carson and H. Wilms. Development of an international standard for shear testing. Powder technology, 167(1):1-9, 2006.

[28] M. E. Cates, J. P. Wittmer, J. P. Bouchaud, and P. Claudin. Jamming, force chains, and fragile matter. Phys. Rev. Lett., 81(9):1841-1844, 1998.

[29] C. S. Chang. Micromechanical modeling of deformation and failure for granulates with frictional contacts. Mechanics of Materials, 16(1-2):13-24, 1993.

[30] C. S. Chang, S. J. Chao, and Y. Chang. Estimates of elastic moduli for granular material with anisotropic random packing structure. Int. J. Solids \& Structures, 32(14):1989-2009, 1995.

[31] C. S. Chang, Q. Shi, and C. L. Liao. Elastic constants for granular materials modeled as first-order straingradient continua. International Journal of Solids and Structures, 40(21):5565-5582, 2003.

[32] P. Charbonneau, E. I. Corwin, G. Parisi, and F. Zamponi. Universal Microstructure and Mechanical Stability of Jammed Packings. Phys. Rev. Lett., 109(20), 2012. 
[33] P. Chaudhuri, L. Berthier, and S. Sastry. Jamming Transitions in Amorphous Packings of Frictionless Spheres Occur over a Continuous Range of Volume Fractions. Phys. Rev. Lett., 104(16):165701, 2010.

[34] G. C. Cho, J.-S. Lee, and J. C. Santamarina. Spatial variability in soils: high resolution assessment with electrical needle probe. Journal of geotechnical and geoenvironmental engineering, 130(8):843-850, 2004.

[35] J. Christoffersen, M. M. Mehrabadi, and Nemat-Nasser. A micro-mechanical description of granular material behavior. Journal of Applied Mechanics, 48(2):339-344, 1981.

[36] C.-K. Chung, E.-R. Jang, S.-H. Baek, and Y.-H. Jung. How contact stiffness and density determine stressdependent elastic moduli: a micromechanics approach. Granular Matter, 16(1):23-39, 2014.

[37] M. P. Ciamarra, M. Nicodemi, and A. Coniglio. Recent results on the jamming phase diagram. Soft Matter, 6(13):2871, 2010.

[38] M. P. Ciamarra, R. Pastore, M. Nicodemi, and A. Coniglio. Jamming phase diagram for frictional particles. Phys. Rev. E, 84:041308, 2011.

[39] F. C. Cosserat. Théorie des Corps Déformables. Paris: Hermann, 1909.

[40] S. C. Cowin. The relationship between the elasticity tensor and the fabric tensor. Mechanics of Materials, 4(2), 1985.

[41] L. Cui and C. O'Sullivan. Exploring the macro- and micro-scale response of an idealised granular material in the direct shear apparatus. Géotechnique, 56(7):455-468, 2006.

[42] P. A. Cundall and O. D. L. Strack. A discrete numerical model for granular assemblies. Géotechnique, 29(1):47-65, 1979.

[43] Y. F. Dafalias and M. T. Manzari. Simple Plasticity Sand Model Accounting for Fabric Change Effects. Journal of Engineering Mechanics, 130(6):622-634, 2004.

[44] S. Dagois-Bohy, B. P. Tighe, J. Simon, S. Henkes, and M. van Hecke. Soft-Sphere Packings at Finite Pressure but Unstable to Shear. Phys. Rev. Lett., 109:095703, 2012.

[45] F. Darve, E. Flavigny, and M. Meghachou. Yield surfaces and principle of superposition: revisit through incrementally non-linear constitutive relations. International Journal of Plasticity, 11(8):927-945, 1995.

[46] O. Dauchot, G. Marty, and G. Biroli. Dynamical Heterogeneity Close to the Jamming Transition in a Sheared Granular Material. Phys. Rev. Lett., 95(26):265701, 2005.

[47] D. Denisov, M. T. Dang, B. Struth, G. Wegdam, and P. Schall. Resolving structural modifications of colloidal glasses by combining X-ray scattering and rheology. Sci. Rep., 3, 2013.

[48] P. J. Digby. The Effective Elastic Moduli of Porous Granular Rocks. Journal of Applied Mechanics, 48(4):803-808, 1981.

[49] P. S. Dodds and J. S. Weitz. Packing-limited growth. Phys. Rev. E, 65(5):056108, 2002.

[50] O. Durán, N. P. Kruyt, and S. Luding. Micro-mechanical analysis of deformation characteristics of threedimensional granular materials. International Journal of Solids and Structures, 47(17):2234-2245, 2010.

[51] Ehlers. From particle ensembles to Cosserat continua: Homogenization of contact forces towards stresses and couple stresses. International Journal of Solids and Structures, 40 (24):6681-6702, 2003.

[52] I. Einav. The unification of hypo-plastic and elasto-plastic theories. International Journal of Solids and Structures, 49(11-12):1305-1315, 2012. 
[53] W. G. Ellenbroek, M. van Hecke, and W. van Saarloos. Jammed frictionless disks: Connecting local and global response. Phys. Rev. E, 80(6):061307, 2009.

[54] B. J. Ennis, J. Green, and R. Davies. The legacy of neglect in the US. Chemical engineering progress, 90(4):32-43, 1994.

[55] P. Eshuis, D. van der Meer, M. Alam, H. J. van Gerner, K. van der Weele, and D. Lohse. Onset of Convection in Strongly Shaken Granular Matter. Phys. Rev. Lett., 104:038001, 2010.

[56] A. Ezaoui and H. Di Benedetto. Experimental measurements of the global anisotropic elastic behaviour of dry Hostun sand during triaxial tests, and effect of sample preparation. Géotechnique, 59(7):621-635, 2009.

[57] O. Farago and Y. Kantor. Fluctuation formalism for elastic constants in hard-spheres-and-tethers systems. Phys. Rev. E, 61(3):2478, 2000.

[58] H. Feise and A. Daiss. Building a numerical model for bulk materials from standard shear test data. Task Quarterly, 7(4):539-547, 2003.

[59] H. Feise and J. Schwedes. Calculation of silo stresses and feeder loads by the hypoplastic material model. Bulk Solids Handling, 16(4):537, 2011.

[60] D. G. Fredlund. Second Canadian Geotechnical Colloquium: Appropriate concepts and technology for unsaturated soils. Canadian Geotechnical Journal, 16(1):121-139, 1979.

[61] F. Froiio and J. N. Roux. Incremental response of a model granular material by stress probing with DEM simulations. In J. Goddard, P. Giovine, and J. T. Jenkins, editors, AIP Conference Proceedings, volume 1227, pages 183-197, Reggio Calabria (Italy), 2010.

[62] R. García-Rojo, F. Alonso-Marroquín, and H. J. Herrmann. Characterization of the material response in granular ratcheting. Phys. Rev. E, 72(4):041302, 2005.

[63] B. S. Gardiner and A. Tordesillas. Micromechanical constitutive modelling of granular media: evolution and loss of contact in particle clusters. Journal of Engineering Mathematics, 52(1), 2005.

[64] J. Goddard. Granular hypoplasticity with Cosserat effects. In J. Goddard, P. Giovine, and J. T. Jenkins, editors, IUTAM-ISIMM Symposium on Mathematical Modeling and Physical Instances of Granular Flows, pages 323-332, Reggio Calabria (Italy), 14-18 September 2009, 2010. AIP.

[65] J. D. Goddard. Nonlinear Elasticity and Pressure-Dependent Wave Speeds in Granular Media. Proc. R. Soc. Lond. A, 430(1878):105-131, 1990.

[66] J. D. Goddard. Continuum Modeling of Granular Assemblies. In H. J. Herrmann, J. P. Hovi, and S. Luding, editors, Physics of dry granular media - NATO ASI Series E 350, pages 1-24, Dordrecht, 1998. Kluwer Academic Publishers.

[67] J. D. Goddard. A dissipative anisotropic fluid model for non-colloidal particle dispersions. Journal of Fluid Mechanics, 568(1):1-17, 2006.

[68] J. D. Goddard. Parametric hypoplasticity as continuum model for granular media: from Stokesium to MohrCoulombium and beyond. Granular Matter, 12(2):145-150, 2010.

[69] F. Göncü, O. Durán, and S. Luding. Constitutive relations for the isotropic deformation of frictionless packings of polydisperse spheres. C. R. Mécanique, 338(10-11):570-586, 2010.

[70] F. Göncü and S. Luding. Effect of particle friction and polydispersity on the macroscopic stress-strain relations of granular materials. Acta Geotechnica, 2013. 
[71] J. Graham and G. T. Houlsby. Anisotropic elasticity of a natural clay. Géotechnique, 33(2):165-180, 1983.

[72] G. Gudehus. A comparison of some constitutive laws for soils under radially symmetric loading and unloading. Canadian Geotechnical Journal, 20:502-516, 1979.

[73] N. Guo and J. Zhao. The signature of shear-induced anisotropy in granular media. Computers and Geotechnics, 47:1-15, 2013.

[74] S. A. Hall, M. Bornert, J. Desrues, Y. Pannier, N. Lenoir, G. Viggiani, and P. Bésuelle. Discrete and continuum analysis of localised deformation in sand using X-ray $\mu \mathrm{CT}$ and volumetric digital image correlation. Géotechnique, 60(5):315-322, 2010.

[75] K. J. Hanley, C. O’Sullivan, E. P. Byrne, and K. Cronin. Discrete element modelling of the quasi-static uniaxial compression of individual infant formula agglomerates. Particuology, 10(5):523-531, 2012.

[76] H. Hareb and T. Doanh. Probing into the strain induced anisotropy of Hostun RF loose sand. Granular Matter, 14(5):589-605, 2012.

[77] R. R. Hartley and R. P. Behringer. Logarithmic rate dependence of force networks in sheared granular materials. Nature, 421(6926):928-931, 2003.

[78] D. L. Henann and K. Kamrin. A predictive, size-dependent continuum model for dense granular flows. Proceedings of the National Academy of Sciences, 110(17):6730-6735, 2013.

[79] H. J. Herrmann and S. Luding. Modeling granular media with the computer. Continuum Mechanics and Thermodynamics, 10:189-231, 1998.

[80] R. C. Hidalgo, C. U. Grosse, F. Kun, H. W. Reinhardt, and H. J. Herrmann. Evolution of Percolating Force Chains in Compressed Granular Media. Phys. Rev. Lett., 89(20), 2002.

[81] A. B. Hopkins, F. H. Stillinger, and S. Torquato. Disordered strictly jammed binary sphere packings attain an anomalously large range of densities. Phys. Rev. E, 88(2), 2013.

[82] S. S. Hsiau, M. H. Wu, and C. H. Chen. Arching phenomena in a vibrated granular bed. Powder technology, 99(2):185-193, 1998.

[83] O. I. Imole, N. Kumar, and S. Luding. Deformation modes of packings of frictionless polydisperse spheres. Particulate Systems Analysis Conference Proceedings, pages 1-6, 2011.

[84] O. I. Imole, N. Kumar, V. Magnanimo, and S. Luding. Hydrostatic and Shear Behavior of Frictionless Granular Assemblies Under Different Deformation Conditions. KONA Powder and Particle Journal, 30:84$108,2013$.

[85] O. I. Imole, M. Wojtkowski, V. Magnanimo, and S. Luding. Force correlations, anisotropy, and friction mobilization in granular assemblies under uniaxial deformation. In A. Yu and S. Luding, editors, Powders and Grains, AIP Conf. Proc., volume 1542, pages 601-604, 2013.

[86] S. Inagaki, M. Otsuki, and S. Sasa. Protocol dependence of mechanical properties in granular systems. The European Physical Journal E, 34(11):1-6, 2011.

[87] G.-L. Jiang, F. Tatsuoka, J. Koseki, and A. Flora. Inherent and stress-state-induced anisotropy in very small strain stiffness of a sandy gravel. Géotechnique, 47(3):509-521, 1997.

[88] Y. Jiang and M. Liu. From Elasticity to Hypoplasticity: Dynamics of Granular Solids. Phys. Rev. Lett., 99:105501, 2007. 
[89] Y. Jiang and M. Liu. Incremental stress-strain relation from granular elasticity: Comparison to experiments. Phys. Rev. E, 77(2), 2008.

[90] Y. Jiang and M. Liu. Granular solid hydrodynamics. Granular Matter, 11(3):139-156, 2009.

[91] I. Jorjadze, L.-L. Pontani, and J. Brujic. Microscopic Approach to the Nonlinear Elasticity of Compressed Emulsions. Phys. Rev. Lett., 110:048302, 2013.

[92] I. Jorjadze, L.-L. Pontani, K. A. Newhall, and J. Brujić. Attractive emulsion droplets probe the phase diagram of jammed granular matter. Proceedings of the National Academy of Sciences, 108(11):4286-4291, 2011.

[93] N. C. Keim and P. E. Arratia. Mechanical and microscopic properties of the reversible plastic regime in a 2D jammed material. Phys. Rev. Lett., 112(2):028302, 2014.

[94] Y. Khidas and X. Jia. Probing the shear-band formation in granular media with sound waves. Phys. Rev. E, 85(5), 2012.

[95] J. B. Knight, C. G. Fandrich, C. N. Lau, H. M. Jaeger, and S. R. Nagel. Density relaxation in a vibrated granular material. Phys. Rev. E, 51(5):3957-3962, 1995.

[96] D. Kolymbas, I. Herle, and P. A. von Wolffersdorff. Hypoplastic constitutive equation with internal variables. International Journal for Numerical and Analytical Methods in Geomechanics, 19(6):415-436, 1995.

[97] D. Krijgsman and S. Luding. 2D cyclic pure shear of granular materials, simulations and model. In S. Luding and A. Yu, editors, Powders \& Grains 2013, pages 1226-1229, Sydney, Australia, 2013. Balkema.

[98] N. P. Kruyt. Statics and kinematics of discrete Cosserat-type granular materials . International Journal of Solids and Structures, 40 (3):511-534, 2003.

[99] N. P. Kruyt, I. Agnolin, S. Luding, and L. Rothenburg. Micromechanical study of elastic moduli of loose granular materials. Journal of the Mechanics and Physics of Solids, 58(9):1286-1301, 2010.

[100] F. Krzakala and J. Kurchan. Landscape analysis of constraint satisfaction problems. Phys. Rev. E., 76(2):021122, 2007.

[101] M. R. Kuhn and K. Bagi. Specimen Size Effect in Discrete Element Simulations of Granular Assemblies. Journal of Engineering Mechanics, 135(6):485-492, 2009.

[102] N. Kumar, O. I. Imole, V. Magnanimo, and S. Luding. Deformation Modes for Assemblies of Frictionless Polydisperse Spheres. Advanced Materials Research, 508:160-165, 2012.

[103] N. Kumar, O. I. Imole, V. Magnanimo, and S. Luding. Effect of polydispersity on the micro-macro behavior of granular assemblies under different deformation paths. Particuology, (http://dx.doi.org/10.1016/j.partic.2013.07.011), 2013.

[104] N. Kumar, O. I. Imole, V. Magnanimo, and S. Luding. Evolution of the Effective Moduli for Anisotropic Granular Materials during Shear. In S. Luding and A. Yu, editors, Powders \& Grains 2013, pages 12381241, Sydney, Australia, 2013. Balkema.

[105] N. Kumar and S. Luding. Memory of jamming and shear-jamming. Submitted, 2014.

[106] N. Kumar, S. Luding, and V. Magnanimo. Macroscopic model with anisotropy based on micro-macro informations. Acta Mechanica, Accepted, 2014.

[107] R. Kuwano and R. J. Jardine. On the applicability of cross-anisotropic elasticity to granular materials at very small strains. Géotechnique, 52(10):727-749, 2002. 
[108] A. Kwade, D. Schulze, and J. Schwedes. Determination of the stress ratio in uniaxial compression tests Part 1. Powder handling \& Processing, 6(1):61-65, 1994.

[109] A. Kwade, D. Schulze, and J. Schwedes. Determination of the stress ratio in uniaxial compression tests Part 2. Powder handling \& Processing, 6(2):199-203, 1994.

[110] L. La Ragione and V. Magnanimo. Contact anisotropy and coordination number for a granular assembly: A comparison of distinct-element-method simulations and theory. Phys. Rev. E., 85(3), 2012.

[111] L. La Ragione, V. Magnanimo, J. Jenkins, and H. Makse. Irreversible Incremental Behavior in a Granular Material. In APS Meeting Abstracts, 2012.

[112] M. Lätzel, S. Luding, H. J. Herrmann, D. W. Howell, and R. P. Behringer. Comparing simulation and experiment of a 2D granular Couette shear device. The European Physical Journal E: Soft Matter and Biological Physics, 11(4):325-333, 2003.

[113] E. Lerner, G. Düring, and M. Wyart. Toward a microscopic description of flow near the jamming threshold. Europhys. Lett., 99(5):58003, 2012.

[114] X. S. Li and Y. F. Dafalias. Constitutive Modeling of Inherently Anisotropic Sand Behavior. Journal of Geotechnical and Geoenvironmental Engineering, 128(10):868-880, 2002.

[115] X. S. Li and Y. F. Dafalias. Anisotropic Critical State Theory: Role of Fabric. Journal of Engineering Mechanics, 138(3):263-275, 2012.

[116] A. J. Liu and S. R. Nagel. Nonlinear dynamics: Jamming is not just cool any more. Nature, 396(6706):21$22,1998$.

[117] A. J. Liu and S. R. Nagel. The Jamming Transition and the Marginally Jammed Solid. Annual Review of Condensed Matter Physics, 1(1):347-369, 2010.

[118] S. Luding. Micro-Macro Models for Anisotropic Granular Media. In P. A. Vermeer, W. Ehlers, H. J. Herrmann, and E. Ramm, editors, Modelling of Cohesive-Frictional Materials, pages 195-206, Leiden, Netherlands, 2004. Balkema.

[119] S. Luding. Micro-macro transition for anisotropic, frictional granular packings. International Journal of Solids and Structures, 41(21):5821-5836, 2004.

[120] S. Luding. Anisotropy in cohesive, frictional granular media. Journal of Physics Condensed Matter, 17(24):S2623-S2640, 2005.

[121] S. Luding. Shear flow modeling of cohesive and frictional fine powder. Powder Technology, 158(1-3):45-50, 2005.

[122] S. Luding. Cohesive, frictional powders: contact models for tension. Granular Matter, 10(4):235-246, 2008.

[123] S. Luding. Constitutive relations for the shear band evolution in granular matter under large strain. Particuology, 6(6):501-505, 2008.

[124] S. Luding. The effect of friction on wide shear bands. Particulate Science Technology, 26(1):33-42, 2008.

[125] S. Luding. Towards dense, realistic granular media in 2D. Nonlinearity, 22(12):R101-R146, 2009.

[126] S. Luding and F. Alonso-Marroqu'in. The critical-state yield stress (termination locus) of adhesive powders from a single numerical experiment. Granular Matter, 13(2):109-119, 2011. 
[127] S. Luding, M. Nicolas, and O. Pouliquen. A minimal model for slow dynamics: Compaction of granular media under vibration or shear. In D. Kolymbas and W. Fellin, editors, Compaction of Soils, Granulates and Powders, pages 241-249, Rotterdam, 2000. A. A. Balkema.

[128] S. Luding and E. S. Perdahcioğlu. A Local Constitutive Model with Anisotropy for Various Homogeneous 2D Biaxial Deformation Modes. Chemie Ingenieur Technik, 83(5):672-688, 2011.

[129] V. Magnanimo, L. La Ragione, J. T. Jenkins, and H. A. Makse. Irreversible incremental behavior in granular materials. In preparation, 2014.

[130] V. Magnanimo, L. La Ragione, J. T. Jenkins, P. Wang, and H. A. Makse. Characterizing the shear and bulk moduli of an idealized granular material. Europhys. Lett., 81(3):34006, 2008.

[131] V. Magnanimo and S. Luding. A local constitutive model with anisotropy for ratcheting under 2D axialsymmetric isobaric deformation. Granular Matter, 13(3):225-232, 2011.

[132] T. S. Majmudar, M. Sperl, S. Luding, and R. P. Behringer. Jamming Transition in Granular Systems. Phys. Rev. Lett., 98(5), 2007.

[133] H. A. Makse, D. L. Johnson, and L. M. Schwartz. Packing of compressible granular materials. Phys. Rev. Lett., 84(18):4160-4163, 2000.

[134] R. Mari, F. Krzakala, and J. Kurchan. Jamming versus Glass Transitions. Phys. Rev. Lett., 103(2):025701, 2009.

[135] Maugin and A. V. Metrikine. Mechanics of generalized continua, volume 21. Springer, 2010.

[136] D. Mašín. Asymptotic behaviour of granular materials. Granular Matter, 14(6):759-774, 2012.

[137] F. Melo, P. B. Umbanhowar, and H. L. Swinney. Hexagons, Kinks, and Disorder in Oscillated Granular Layers. Phys. Rev. Lett., 75(21):3838-3841, 1995.

[138] E. Merrow. Estimating startup times for solids-processing plants . Chemical engineering, 95:89-92, 1988.

[139] J. F. Métayer, D. J. Suntrup, C. Radin, H. L. Swinney, and M. Schröter. Shearing of frictional sphere packings. Europhys. Lett., 93(6):64003, 2011.

[140] G. D. R. MiDi. On dense granular flows. The European Physical Journal E: Soft Matter and Biological Physics, 14(4):341-365, 2004.

[141] M. Morgeneyer and J. Schwedes. Investigation of powder properties using alternating strain paths. Task Quarterly, 7(4):571-578, 2003.

[142] H. Muhlhaus, L. Moresi, L. Gross, and J. Grotowski. The influence of non-coaxiality on shear banding in viscous-plastic materials. Granular Matter, 12:229-238, 2010.

[143] S. Nemat-Nasser. A micromechanically-based constitutive model for frictional deformation of granular materials. Journal of the Mechanics and Physics of Solids, 48(6-7):1541-1563, 2000.

[144] F. Nicot and F. Darve. Basic features of plastic strains: From micro-mechanics to incrementally nonlinear models. International Journal of Plasticity, 23(9):1555-1588, 2007.

[145] F. Nicot and F. Darve. Micro-mechanical bases of some salient constitutive features of granular materials. International Journal of Solids and Structures, 44(22-23):7420-7443, 2007.

[146] M. Oda. Initial fabrics and their relations to mechanical properties of granular materials. Soils and Foundation, 1(12):17-36, 1972. 
[147] V. Ogarko and S. Luding. Equation of state and jamming density for equivalent bi- and polydisperse, smooth, hard sphere systems. Journal of Chemical Physics, 136(12), 2012.

[148] C. S. O’Hern, S. A. Langer, A. J. Liu, and S. R. Nagel. Force Distributions near Jamming and Glass Transitions. Phys. Rev. Lett., 86(1):111-114, 2001.

[149] C. S. O’Hern, S. A. Langer, A. J. Liu, and S. R. Nagel. Random packings of frictionless particles. Phys. Rev. Lett., 88(7), 2002.

[150] C. S. O'Hern, L. E. Silbert, A. J. Liu, and S. R. Nagel. Jamming at zero temperature and zero applied stress: The epitome of disorder. Phys. Rev. E, 68(1, Part 1), 2003.

[151] P. Olsson and S. Teitel. Critical scaling of shearing rheology at the jamming transition of soft-core frictionless disks. Phys. Rev. E., 83(3):030302, 2011.

[152] P. Olsson and S. Teitel. Athermal jamming versus thermalized glassiness in sheared frictionless particles. Phys. Rev. E, 88(1), 2013.

[153] M. Otsuki and H. Hayakawa. Critical scaling near jamming transition for frictional granular particles. Phys. Rev. E, 83(5), 2011.

[154] M. Otsuki and H. Hayakawa. Critical scaling of a jammed system after a quench of temperature. Phys. Rev. E, 86(3):031505, 2012.

[155] M. Ozawa, T. Kuroiwa, A. Ikeda, and K. Miyazaki. Jamming Transition and Inherent Structures of Hard Spheres and Disks. Phys. Rev. Lett., 109(20):205701, 2012.

[156] Pardem. PARticle Systems: Training on DEM Simulation for Industrial and Scientific Applications. pages 1-41, 2008.

[157] G. Parisi and F. Zamponi. Mean-field theory of hard sphere glasses and jamming. Rev. Mod. Phys., 82(1):789-845, 2010.

[158] P.-E. Peyneau and J.-N. Roux. Frictionless bead packs have macroscopic friction, but no dilatancy. Phys. Rev. E, 78:011307, 2008.

[159] P.-E. Peyneau and J.-N. Roux. Solidlike behavior and anisotropy in rigid frictionless bead assemblies. Phys. Rev. E, 78(4), 2008.

[160] M. Pica Ciamarra and A. Coniglio. Jamming at Zero Temperature, Zero Friction, and Finite Applied Shear Stress. Phys. Rev. Lett., 103(23), 2009.

[161] F. Radjaï, S. Roux, and J. J. Moreau. Contact forces in a granular packing. Chaos, 9(3):544-550, 1999.

[162] F. Radjai, D. E. Wolf, M. Jean, and J. Moreau. Bimodal Character of Stress Transmission in Granular Packings. Phys. Rev. Lett., 80:61-64, 1998.

[163] J. Ren, J. A. Dijksman, and R. P. Behringer. Reynolds Pressure and Relaxation in a Sheared Granular System. Phys. Rev. Lett., 110:018302, 2013.

[164] L. Ren, J. Tong, D. Chen, D. He, Z. Han, and J. Li. Conductive percolation threshold of conductive-insulating granular composites. Journal of Materials Science, 41:2157-2159, 2006.

[165] P. Richard, M. Nicodemi, R. Delannay, P. Ribiere, and D. Bideau. Slow relaxation and compaction of granular systems. Nat Mater, 4(2), 2005. 
[166] M. Röck, M. Morgeneyer, J. Schwedes, D. Kadau, L. Brendel, and D. E. Wolf. Steady state flow of cohesive and non-cohesive powders. Granular Matter, 10(4):285-293, 2008.

[167] A. D. Rosato, O. Dybenko, D. J. Horntrop, V. Ratnaswamy, and L. Kondic. Microstructure evolution in density relaxation by tapping. Phys. Rev. E, 81:061301, 2010.

[168] A. D. Rosato, K. J. Strandburg, F. Prinz, and R. H. Swendsen. Why the Brazil nuts are on top: Size segregation of particulate matter by shaking. Phys. Rev. Lett., 58(10):1038, 1987.

[169] K. H. Roscoe, A. N. Schofield, and C. P. Wroth. On The Yielding of Soils. Géotechnique, 8(1):22-53, 1958.

[170] M. Saadatfar, A. P. Sheppard, T. J. Senden, and A. J. Kabla. Mapping forces in a 3D elastic assembly of grains. Journal of the Mechanics and Physics of Solids, 60(1):55-66, 2012.

[171] K. Saitoh, V. Magnanimo, and S. Luding. Master equation for the probability distribution functions of overlaps between particles in two dimensional granular packings. arXiv:1311.5359, 2014.

[172] A. Samimi, A. Hassanpour, and M. Ghadiri. Single and bulk compressions of soft granules: Experimental study and DEM evaluation. Chemical Engineering Science, 60(14):3993-4004, 2005.

[173] M. Satake. Fabric tensor in granular materials. Proc., IUTAM Symp. on Deformation and Failure of Granular materials, Delft, The Netherlands, 1982.

[174] P. Schall and M. van Hecke. Shear bands in matter with granularity. Annual Review of Fluid Mechanics, 42(1):67, 2009.

[175] G. E. Schröder-Turk, W. Mickel, M. Schröter, G. W. Delaney, M. Saadatfar, T. J. Senden, K. Mecke, and T. Aste. Disordered spherical bead packs are anisotropic. Europhys. Lett., 90(3):34001, 2010.

[176] J. Schwedes. Review on testers for measuring flow properties of bulk solids. Granular Matter, 5(1):1-43, 2003.

[177] V. M. Segal. Severe plastic deformation: simple shear versus pure shear. Materials Science and Engineering: A, 338(1-2):331-344, 2002.

[178] M. R. Shaebani, J. Boberski, and D. E. Wolf. Unilateral interactions in granular packings: a model for the anisotropy modulus. Granular Matter, 14(2):265-270, 2012.

[179] M. R. Shaebani, M. Madadi, S. Luding, and D. E. Wolf. Influence of polydispersity on micromechanics of granular materials. Phys. Rev. E, 85(1), 2012.

[180] L. Sibille, F. Nicot, F.-V. Donzé, and F. Darve. Analysis of failure occurrence from direct simulations. European Journal of Environmental and Civil Engineering, 13(2):187-201, 2009.

[181] L. E. Silbert. Jamming of frictional spheres and random loose packing. Soft Matter, 6:2918-2924, 2010.

[182] L. E. Silbert, A. J. Liu, and S. R. Nagel. Structural signatures of the unjamming transition at zero temperature. Phys. Rev. E, 73(4, Part 1), 2006.

[183] L. E. Silbert, A. J. Liu, and S. R. Nagel. Normal modes in model jammed systems in three dimensions. Phys. Rev. E, 79:021308, 2009.

[184] K. C. Smith, T. S. Fisher, and M. Alam. Isostaticity of constraints in amorphous jammed systems of soft frictionless Platonic solids. Phys. Rev. E, 84:030301, 2011.

[185] J. H. Snoeijer, W. G. Ellenbroek, T. J. H. Vlugt, and M. van Hecke. Sheared Force Networks: Anisotropies, Yielding, and Geometry. Phys. Rev. Lett., 96:098001, 2006. 
[186] E. Somfai, M. van Hecke, W. G. Ellenbroek, K. Shundyak, and W. van Saarloos. Critical and noncritical jamming of frictional grains. Phys. Rev. E, 75(2):020301, 2007.

[187] C. Song, P. Wang, and H. A. Makse. A phase diagram for jammed matter. Nature, 453(7195), 2008.

[188] M. Sperl. Experiments on corn pressure in silo cells. Translation and comment of Janssen's paper from 1895. Granular Matter, 8(2):59-65, 2006.

[189] J. Sun and S. Sundaresan. A plasticity model with microstructure evolution for dense granular flows. AIP Proceeding for the Joint IUTAM-ISIMM Symposium on Mathematical Modeling and Physical Instances of Granular Flows, 1227:280-289, 2010.

[190] J. Sun and S. Sundaresan. A constitutive model with microstructure evolution for flow of rate-independent granular materials. Journal of Fluid Mechanics, 682:590-616, 2011.

[191] T-Mappp. Training in Multiscale Analysis of multiPhase Particulate Processes. pages 1-45, 2008.

[192] J. Tejchman and E. Bauer. Effect of cyclic shearing on shear localisation in granular bodies. Granular Matter, 5(4):201-212, 2004.

[193] J. Tejchman, E. Bauer, and W. Wu. Effect of fabric anisotropy on shear localization in sand during plane strain compression. Acta Mechanica, 189(1-4):23-51, 2007.

[194] J. Tejchman and W. Wu. Modeling of textural anisotropy in granular materials with stochastic micro-polar hypoplasticity. International Journal of Non-Linear Mechanics, 42(6):882-894, 2007.

[195] C. Thornton. Quasi-static simulations of compact polydisperse particle systems. Particuology, 8(2):119-126, 2010.

[196] C. Thornton and L. Zhang. A numerical examination of shear banding and simple shear non-coaxial flow rules. Philosophical Magazine, 86(21-22):3425-3452, 2006.

[197] C. Thornton and L. Zhang. On the evolution of stress and microstructure during general 3D deviatoric straining of granular media. Géotechnique, 60(5):333-341, 2010.

[198] S. Torquato, T. M. Truskett, and P. G. Debenedetti. Is random close packing of spheres well defined? Phys. Rev. Lett., 84(10):2064-2067, 2000.

[199] V. Trappe, V. Prasad, L. Cipelletti, P. N. Segre, and D. A. Weitz. Jamming phase diagram for attractive particles. Nature, 411(6839):772-775, 2001.

[200] M. van Hecke. Jamming of soft particles: geometry, mechanics, scaling and isostaticity. Journal of Physics: Condensed Matter, 22:033101(3), 2009.

[201] P. A. Vermeer, S. Diebels, W. Ehlers, H. J. Herrmann, S. Luding, and E. Ramm, editors. Continuous and Discontinuous Modelling of Cohesive Frictional Materials, Berlin, 2001. Springer.

[202] C. Voivret, F. Radjaï, J. Y. Delenne, and M. S. El Youssoufi. Space-filling properties of polydisperse granular media. Phys. Rev. E, 76(2), 2007.

[203] C. Voivret, F. Radjaï, J. Y. Delenne, and M. S. El Youssoufi. Multiscale Force Networks in Highly Polydisperse Granular Media. Phys. Rev. Lett., 102(17), 2009.

[204] D. Vågberg, P. Olsson, and S. Teitel. Glassiness, rigidity, and jamming of frictionless soft core disks. Phys. Rev. E., 83(3):031307, 2011. 
[205] S. D. C. Walsh and A. Tordesillas. A thermomechanical approach to the development of micropolar constitutive models of granular media. Acta mechanica, 167(3):145-169, 2004.

[206] K. Walton. The effective elastic moduli of a random packing of spheres. Journal of the Mechanics and Physics of Solids, 35(2):213-226, 1987.

[207] J. Wambaugh, R. Behringer, J. Matthews, and P. Gremaud. Response to perturbations for granular flow in a hopper. Phys. Rev. E, 76(5):051303, 2007.

[208] R. Wan, F. Nicot, and F. Darve. Micromechanical Formulation of Stress Dilatancy as a Flow Rule in Plasticity of Granular Materials. Journal of Engineering Mechanics, 136(5):589-598, 2010.

[209] K. Wang, C. Song, P. Wang, and H. A. Makse. Edwards thermodynamics of the jamming transition for frictionless packings: Ergodicity test and role of angoricity and compactivity. Phys. Rev. E, 86:011305, 2012.

[210] X. Wang, H. P. Zhu, S. Luding, and A. B. Yu. Regime transitions of granular flow in a shear cell: A micromechanical study. Phys. Rev. E, 88(3):032203, 2013.

[211] Y. H. Wang and C. M. Mok. Mechanisms of Small-Strain Shear-Modulus Anisotropy in Soils. Journal of Geotechnical and Geoenvironmental Engineering, 134(10):1516-1530, 2008.

[212] T. Weinhart, A. R. Thornton, S. Luding, and O. Bokhove. From discrete particles to continuum fields near a boundary. Granular Matter, 14(2):289-294, 2012.

[213] W. Wu. Rational approach to anisotropy of sand. Int. J. for Numerical and Analytical Methods in Geomechanics, 22(11), 1998

[214] N. Xu, D. Frenkel, and A. J. Liu. Direct Determination of the Size of Basins of Attraction of Jammed Solids. Phys. Rev. Lett., 106(24), 2011.

[215] S. Yimsiri and K. Soga. DEM analysis of soil fabric effects on behaviour of sand. Géotechnique, 60(6):483495,2010

[216] M. Yoshimine, K. Ishihara, and W. Vargas. Effects of principal stress direction and intermediate principal stress on undrained shear behavior of sand. Soils and Foundations, 38(3):179-188, 1998.

[217] H. Zetzener and J. Schwedes. Deformation behaviour and relaxation of bulk solids at different deformation rates. 6th Intl. Conf. on Handling and Transportation, pages 51-55, 1998.

[218] H. P. Zhang and H. A. Makse. Jamming transition in emulsions and granular materials. Phys. Rev. E, 72:011301, 2005.

[219] J. Zhang, T. Majmudar, A. Tordesillas, and R. Behringer. Statistical properties of a 2D granular material subjected to cyclic shear. Granular Matter, 12(2):159-172, 2010.

[220] J. Zhang, T. S. Majmudar, M. Sperl, and R. P. Behringer. Jamming for a 2D granular material. Soft Matter, 6(13):2982-2991, 2010.

[221] C. Zhao, K. Tian, and N. Xu. New Jamming Scenario: From Marginal Jamming to Deep Jamming. Phys. Rev. Lett., 106(12):125503, 2011.

[222] J. Zhao and N. Guo. Unique critical state characteristics in granular media considering fabric anisotropy. Géotechnique, 63(8):695-704, 2013.

[223] X. Zhao and T. M. Evans. Numerical analysis of critical state behaviors of granular soils under different loading conditions. Granular Matter, 13(6):751-764, 2011. 


\section{Curriculum vitae}

\section{Nishant Kumar}

Born on July 6, 1987 in Allahabad, India

June 2004

High school diploma, Kendriya Vidyala, Phulpur

August 2008

Bachelor of Technology in Chemical Engineering, IIT Kanpur

May - July 2007

Internship, Laboratory of Physics of condensed matter, France

Oct. - Dec. 2009

Internship, Nestle Research center (NRC), Switzerland

June-Sept. 2009, '10

Internship, Schlumberger Doll Research (SDR), Boston

March 2010

Master of Science in Mechanical Engineering, University of California, San Diego

Jan 2013

Minor in Innovation and Entrepreneurship, University of Twente

August 2010 - Present Researcher, University of Twente

\section{Publications}

Journal Papers

1. O. I. Imole, N. Kumar, V. Magnanimo, and S. Luding. Hydrostatic and Shear Behavior of Frictionless Granular Assemblies Under Different Deformation Conditions. KONA Powder and Particle Journal, 30:84-108, 2013

2. N. Kumar, O. I. Imole, V. Magnanimo, and S. Luding. Effect of polydispersity on the micro-macro behavior of granular assemblies under different deformation paths. Particuology, (http://dx.doi.org/10.1016/j.partic.2013.07.011), 2013

3. N. Kumar, S. Luding, and V. Magnanimo. Macroscopic model with anisotropy based on micro-macro informations. Acta Mechanica, Accepted, 2014

4. N. Kumar and S. Luding. Memory of jamming and shear-jamming. Submitted, 2014 
Conference Proceedings

5. N. Kumar, O. I. Imole, V. Magnanimo, and S. Luding. Evolution of the Effective Moduli for Anisotropic Granular Materials during Shear. In S. Luding and A. Yu, editors, Powders \& Grains 2013, pages 1238-1241, Sydney, Australia, 2013. Balkema

6. N. Kumar, O. I. Imole, V. Magnanimo, and S. Luding. Deformation Modes for Assemblies of Frictionless Polydisperse Spheres. Advanced Materials Research, 508:160 165,2012

7. O. I. Imole, N. Kumar, and S. Luding. Deformation modes of packings of frictionless polydisperse spheres. Particulate Systems Analysis Conference Proceedings, pages $1-6,2011$

Seminars and Presentations

1. 16th EM Symposium, Lunteren, The Netherlands, October 2013

2. 3rd International Conference on Particle-Based Methods (PARTICLES 2013), Stuttgart, Germany, September 2013

3. 6th International Conference on Discrete Element Methods (DEM6), Golden, Colorado, USA, August 2013

4. Powders \& Grains 2013, Sydney, Australia, July 2013

5. 7th International Conference for Conveying and Handling of Particulate Solids (CHoPS), Friedrichshafen, Germany, September 2012

6. 8th European Solid Mechanics Conference (ESMC), Graz, Austria, July 2012

7. 5th Asian Particle Technology Symposium, National University of Singapore, Singapore, July 2012

8. Particulate Systems Analysis (PSA), Edinburgh, UK, September 2011

9. Marie Curie Researchers Symposium (poster), Warsaw, Poland, September 2011

10. Soft Condensed Matter Physics, Gordon Research Conference (GRC), New London, USA, August 2011

11. Physics@FOM (poster), Veldhoven, The Netherlands, Januray 2011 